\title{
Patterning Nanocrystals Using DNA
}

\author{
by \\ Shara Carol Williams \\ B.S. (Stanford University) 1997 requirements for the degree of Doctor of Philosophy
in
Chemistry
in the
GRADUATE DIVISION
of the
UNIVERSITY OF CALIFORNIA, BERKELEY \\ A dissertation submitted in partial satisfaction of the \\ Committee in charge: \\ Professor A. Paul Alivisatos, Chair \\ Professor Herbert L. Strauss \\ Professor Arunava Majumdar
}

Fall 2003 
The dissertation of Shara Carol Williams is approved:

\begin{tabular}{lc}
\hline Chair & Date \\
& \\
\hline & Date \\
\hline & \\
\hline
\end{tabular}

University of California, Berkeley

Fall 2003 


\section{Patterning Nanocrystals Using DNA}

Copyright 2003

by

Shara Carol Williams 


\begin{abstract}
Patterning Nanocrystals Using DNA

by

Shara Carol Williams

Doctor of Philosophy in Chemistry

University of California, Berkeley

Professor A. Paul Alivisatos, Chair
\end{abstract}

One of the goals of nanotechnology is to enable programmed self-assembly of patterns made of various materials with nanometer-sized control. This dissertation describes the results of experiments templating arrangements of gold and semiconductor nanocrystals using 2'deoxyribonucleic acid (DNA). Previously, simple DNA-templated linear arrangements of two and three nanocrystals structures have been made.[1] Here, we have sought to assemble larger and more complex nanostructures. Gold-DNA conjugates with 50 to 100 bases selfassembled into planned arrangements using strands of DNA containing complementary base sequences. We used two methods to increase the complexity of the arrangements: using branched synthetic doublers within the DNA covalent backbone to create discrete nanocrystal groupings, and incorporating the nanocrystals into a previously developed DNA lattice structure [2][3] that self-assembles from tiles made of DNA double-crossover molecules to create ordered nanoparticle arrays. In the first project, the introduction of a covalently- 
branched synthetic doubler reagent into the backbone of DNA strands created a branched DNA "trimer." This DNA trimer templated various structures that contained groupings of three and four gold nanoparticles, giving promising, but inconclusive transmission electron microscopy (TEM) results. Due to the presence of a variety of possible structures in the reaction mixtures, and due to the difficulty of isolating the desired structures, the TEM and gel electrophoresis results for larger structures having four particles, and for structures containing both 5 and $10 \mathrm{~nm}$ gold nanoparticles were inconclusive. Better results may come from using optical detection methods, or from improved sample preparation. In the second project, we worked toward making two-dimensional ordered arrays of nanocrystals. We replicated and improved upon previous results for making DNA lattices, increasing the size of the lattices to a length greater than $20 \mu \mathrm{m}$, and collecting atomic force microscopy (AFM) images up to $30 \mu \mathrm{m}$. We found the lattices' requirement of divalent magnesium cations to stabilize Holliday junctions to be incompatible with the stability of charge-stabilized gold nanoparticles used for the experiments here, and gold particles added indiscriminately to the lattice surface through non-specific binding. Redesigning the lattices to avoid magnesium may improve results.

Professor A. Paul Alivisatos Dissertation Committee Chair 
To my family,

whose enthusiasm and faith in me

gave strength to my conviction, and courage to my heart. 


\section{Contents}

List of Figures $\quad$ iv

List of Tables $\quad$ XV

1 Motivation and Background 1

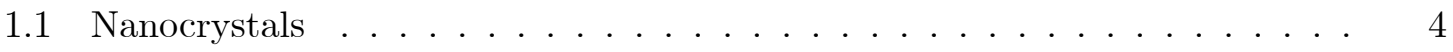

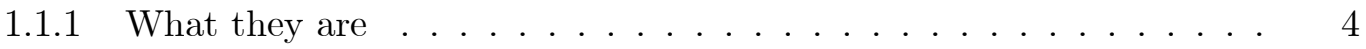

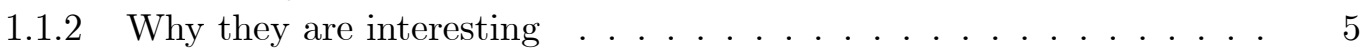

1.1.3 Types of nanocrystals we used . . . . . . . . . . . 5

1.2 Reasons for Making Nanocrystal Arrangements . . . . . . . . . . . . . . 7

1.3 DNA as a Template Material for Nanocrystals . . . . . . . . . . . . . . 9

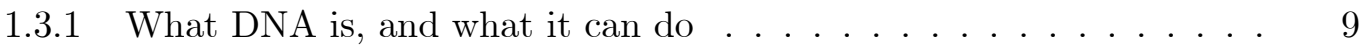

1.3.2 DNA in nanotechnology . . . . . . . . . . . . . . 12

1.4 Benefits of Using DNA to Organize Nanocrystals . . . . . . . . . . . . . 14

1.4.1 Self-assembly . . . . . . . . . . . . . . . . . . 14

1.4 .2 Programmability . . . . . . . . . . . . . . . . 16

1.4 .3 Distance control . . . . . . . . . . . . . . . . . . 17

1.4.4 Control of surface location . . . . . . . . . . . . 17

2 Sample Preparation and Characterization $\quad 18$

2.1 Gold Nanocrystal Synthesis . . . . . . . . . . . . . . . . . . . . 18

2.2 Semiconductor Nanocrystal Synthesis _ . . . . . . . . . . . . . 21

2.3 DNA Synthesis . . . . . . . . . . . . . . . . . . 29

2.3 .1 DNA purification . . . . . . . . . . . . . . 31

2.3 .2 Gel electrophoresis . . . . . . . . . . . . . . . . . . 33

2.3 .3 MALDI . . . . . . . . . . . . . . . . . . . 49

2.3.4 Unnatural synthetic modifications of DNA . . . . . . . . . 52

3 Using DNA Trimers to Arrange Gold Nanocrystals 55

3.1 Motivation . . . . . . . . . . . . . . . . . 55

3.2 Structure and Sequence Design . . . . . . . . . . . . . . . . 61

3.3 Nanocrystal-DNA Conjugate Preparation . . . . . . . . . . . . . 66 
3.4 DNA Trimer Synthesis . . . . . . . . . . . . . . . . . . . . . 74

3.5 Project Conclusions . . . . . . . . . . . . . . . . . . . 102

4 Progress Toward Using DNA Lattices to Pattern Nanocrystals 104

4.1 Motivation . . . . . . . . . . . . . . . . . 104

4.2 Previous Work . . . . . . . . . . . . . . . 105

4.3 Choice of Lattice System . . . . . . . . . . . . . . . . . . . . 106

4.4 Lattices and Nanocrystal Compatibility . . . . . . . . . . . . . . . . 109

4.5 Making Lattices . . . . . . . . . . . . . . . . . . . . . . . . . . . . . . . . . . . . . . . . . . .

4.6 Characterizing Lattices . . . . . . . . . . . . . . . . . . 120

4.7 Factors Influencing Increased Lattice Size . . . . . . . . . . . . . . . 143

4.8 Factors Influencing Successful Nanocrystal Patterning . . . . . . . . . . . 143

4.9 Results of Patterning Attempts . . . . . . . . . . . . . . . . . . 145

4.10 Future Work on Patterning Nanocrystals on Lattices . . . . . . . . . . . . . 149

4.11 Project Conclusions . . . . . . . . . . . . . . . . . . 150

5 Conclusion $\quad 152$

$\begin{array}{lr}\text { Bibliography } & 154\end{array}$

$\begin{array}{ll}\text { A Glossary } & 164\end{array}$

B List of Publications and Presentations $\quad 175$ 


\section{List of Figures}

1.1 This figure from Dabbousi, et. al. shows a selection of six different sizes of $\mathrm{CdSe} / \mathrm{ZnS}$ core/shell nanocrystals under excitation with a $365 \mathrm{~nm}$ source luminescing in a range of wavelengths between 470 and $620 \mathrm{~nm}$, left to right.[4] 7

1.2 2'-Deoxyribose joined by phosphodiester linkages forms a polymer of sugars to make DNA. . . . . . . . . . . . . . . . . . .

1.3 Model of Watson-Crick base pairs in DNA. Juergen Suehnel, Image Library of Macromolecules, Jena/Germany.[5] . . . . . . . . . . . . . . . .

1.4 Two strands of DNA with complementary base sequences hydrogen-bond to form a double helix. Juergen Suehnel, Image Library of Macromolecules, Jena/Germany. [5] . . . . . . . . . . . . . . . . . . . . . .

1.5 Cartoon showing formation of self-assembled structure. Atoms first combine to make defined building blocks. These building blocks are designed so that their properties cause them to self-assemble to form a large, non-covalently bound structure that is the thermodynamic equilibrium assembly for the starting block materials. $[6] \ldots \ldots \ldots \ldots \ldots$

2.1 Absorption spectra of 5 and $10 \mathrm{~nm}$ gold nanoparticles coated with phosphine ligand, dissolved in water. . . . . . . . . . . . . . . . .

2.2 This figure shows the typical setup for performing synthesis of CdSe nanocrystals. We added the outer ZnS shell, subsequently, on the same assembly. 22

2.3 A sketch of the CdSe core and ZnS shell complete synthesis setup, attached to a Schlenk line that provided access to both vacuum and argon. . . . . . .

2.4 Diagram showing the steps for creating a siloxane layer on the outside of core/shell nanocrystals. In the first step, mercaptopropylsilane primes the surface of TOPO-coated nanocrystals. Then base hydrolyzes the silanol groups to form siloxane bridges. The reaction can incorporate different functional groups ("X") in the outside coating of the layer in the final step by addition of an appropriate reagent. . . . . . . . . . . . . 
2.5 Figure from Gerion, et. al. [7] showing the absorption and emission spectra of a set of red, orange, yellow, green, and blue silanized CdSe/ZnS nanocrystals. The upper half of the figure contains absorption spectra, and the lower half contains emission spectra. The top-right inset shows an example of the very small difference for the absorption and emission profiles in a sample of green fluorescencing nanocrystals, before (in dashed lines) and after (in solid lines) siloxane coating. . . . . . . . . . . . . . . . . .

2.6 Steps for running a DNA polyacrylamide vertical gel. (from Karp: Cell and Molecular Biology $[8$, p.762] . . . . . . . . . . . . . .

2.7 Polyacrylamide gel running in a temperature-controlled buffer bath. The blue bands seen in the gel are two kinds of dyes: xylene cyanol FF (top band), and bromophenol blue (bottom band) that are merely markers that allow the user to track the progress of the gel run. . . . . . . . . .

2.8 Example of a DNA-containing, 20\% denaturing PAGE gel showing four lanes loaded (lane 1, 20 bp ladder (denatured); lane 2, an 18 base single strand; lane 3, a 36 base strand; lane 4 , a 54 base strand). . . . . . . . . . . . . .

2.9 Purification of 54 base DNA in $15 \%$ denaturing PAGE. The top image shows the gel before cutting, and the bottom shows the excised band. The bands below the excised band are the shorter failure contaminants of the synthesis reaction that we are trying to remove. (The line across the top gel was a fracture caused by the process of transfering the gel onto the transilluminator for imaging. It did not affect the results.) . . . . . . . . . .

2.10 Array of gels comparing purity of synthesized DNA before and after PAGE purification. $1 \mu \mathrm{g}$ of DNA was loaded per well. Adjacent wells have purified and raw samples of the same strand. Numbers above each set of brackets refer the length in bases of the purified strands. All gels were denaturing PAGE: "A," "B," "C, "D," "E," and "F" were $20 \%$ gels. "G" was $11 \%$. Gels were run at $50{ }^{\circ} \mathrm{C}$ at $500 \mathrm{~V}$. Run times were adjusted to allow appropriate resolution of bands. These results show all the strands had been adequately purified. . . . . . . . . . . . . . . . . .

2.11 12\% native PAGE gel showing proper double helix formation by an unusual three-branched DNA strand. The right-most three lanes show higher, more retarded, bands that correspond to addition of complementary DNA to the structure through duplex formation. This $16 \mathrm{~cm}$ gel was run at $4^{\circ} \mathrm{C}$ at 160 $\mathrm{V}$ in $1 \mathrm{X}$ TBE for $\sim 5 \mathrm{hrs} . \ldots \ldots \ldots \ldots$

2.12 A diagram of a horizontal agarose gel electrophoresis setup, showing a gel submersed in buffer, and connected to a power supply. . . . . . . . . .

2.13 An agarose DNA separation gel. This native 5\% "Metaphor" agarose gel was run at $75 \mathrm{~V}$ for $2 \mathrm{hr}$. Lanes contain, left to right, a $20 \mathrm{bp}$ ladder, a 50 mer, the 50 mer + an 18 base complement, and the 50 mer + the 18 mer with an attached second-generation dendrimer. . . . . . . . . .

2.14 Two types of agarose electrophoresis units with gold nanoparticle samples. The top image shows a horizontal agarose gel box, and the bottom shows a preparative tube gel. . . . . . . . . . . . . . . . 
2.15 The entire preparative gel setup, with the attached fraction collector and pump, and an additional pump that provides cooling. (Purchased from Bio-Rad). . . . . . . . . . . . . . . . . .

2.16 Shown on the left, the large ribosomal subunit, composed of RNA and protein, from the prokaryote, Haloarcula marismortui, [9] which is approximately $25 \mathrm{~nm}$ across; on the right, a proportionately scaled $10 \mathrm{~nm}$ gold nanoparticle. 48

2.17 Spliced image of a single $10 \mathrm{~cm}$ agarose gel showing the migration of $5 \mathrm{~nm}$ gold compared to $10 \mathrm{~nm}$ gold coated with phosphine ligand. . . . . . . . . .

2.18 Three samples of yellow core/shell CdSe/ZnS nanocrystals coated with a siloxane layer, as described in Section 1.1.3. From left to right, the first lane contains nanocrystals that were primed with mercaptopropylsilane (MPS), then dialyzed against basified methanol, then subsequently coated with polyethylene glycol and aminopropylsilane (APS). The second lane had only MPS in place of the APS, and the third lane had a blend of $20 \%$ APS and $80 \%$ MPS. The agarose gel was run in $0.5 \mathrm{X}$ TBE at $100 \mathrm{~V}$ for $45 \mathrm{~min}$ by Dr. Daniele Gerion. . . . . . . . . . . . . . . . . . . . .

2.19 A laser pulse impacts the surface where the DNA analyte and the matrix have co-crystallized for MALDI analysis. (After a figure from srsmaldi.com.)[10]

2.20 Observed MALDI spectrum for 18 base linear DNA. The top number is the resulting molecular weight. The expected molecular weight for the molecule is shown in parentheses. The result is within acceptable error for the instrument. . . . . . . . . . . . . . . . . .

2.21 Doubling synthon phosphoramidite purchased from Glen Research, as described in the 1999 Nucleic Acids Research paper by Southern, et. al.[11] .

3.1 DNA links two sizes of gold nanoparticles into discrete structures visible under TEM. (Results from [1].) . . . . . . . . . . . . . . .

3.2 Cartoon showing the design features for the target gold trimer structure containing an asymmetric, branched, DNA trimer. The identity of DNA sequence is keyed to color. Two arms of the structure contain the same sequences. The third arm is unique. . . . . . . . . . . . .

3.3 Addition of thiolated DNA to gold nanoparticles yields a mixture of reaction products that have dispersity in the number of DNA strands added to the gold. The number present of any particular type of conjugate depends on the stoichiometry of the DNA/Au ratio. . . . . . . . . . . . .

3.4 Addition of the unpurified reaction products from Au-DNA conjugate preparations of complementary DNA strands will result in uncontrolled aggregation. The resulting structures will be clumps of gold particles without specific order, regardless of the claims of Prof. Mirkin and others.[12] . . . . 
3.5 Titration of 100 base thiolated DNA addition to $10 \mathrm{~nm}$ gold particles. The leftmost lane has $2 \mathrm{pmol}$ of phosphine-coated gold sample. The next three lanes have 2:1, 3:1, and 4:1 ratios, respectively, of DNA to gold. The resulting bands in the gel are labeled according to the type of conjugate they contain. The efficiency of DNA addition to gold varied widely from sample to sample. It was not possible to quantitate the DNA on the gold by UV/Vis spectroscopy because the gold itself absorbs heavily at the relevant $260 \mathrm{~nm}$ DNA base peak. (See Figure 2.1.) Poor DNA or gold samples led to more diffuse bands in the agarose gel. . . . . . . . . . . . . . . . . .

3.6 Flow chart describing the process contained in Prof. Nadrian Seeman's "Sequin" program.[13] . . . . . . . . . . . . . . . .

3.7 Series of four simultaneous conjugate titrations reacted for about two hours before gel loading. From left to right, lanes 1-4 show 50 base 3'-thiolated DNA sequence "A" added to $5 \mathrm{~nm}$ gold. Lane 1 is gold only, 2 has gold +0.33 eq. of DNA, 3 has gold +0.66 eq. of DNA, and 4 has gold +1 equivalent of DNA. Lanes 5-8 and 9-12 show addition of the same DNA to two other samples of gold, with lanes 5 and 9 having plain gold, and each successive lane having 1, 2 and 3 equivalents of DNA, respectively. The four rightmost lanes show addition of 50 base 3'-thiolated DNA sequence "B" to $10 \mathrm{~nm}$ gold. Lane 13 has unconjugated $10 \mathrm{~nm}$ gold, and lanes 14-16 have 1,2 , and 3 equivalents, of DNA respectively. Unmodified lanes were loaded with $2 \mathrm{pmol}$ of $10 \mathrm{~nm}$ gold or $20 \mathrm{pmol}$ of $5 \mathrm{~nm}$ gold. Conjugate lanes were loaded with $4 \mathrm{pmol}$ of $10 \mathrm{~nm}$ gold, or 35-40 pmol of $5 \mathrm{~nm}$ gold. Loading heavily impacts the visibility and resolution of bands. . . . . . . . . . .

3.8 Two gels out of eight containing the products of a preparatory reaction of 10 nm gold nanoparticles with 50 base DNA (sequence "B"). ${ }^{3} \ldots \ldots$. . . . .

$3.910 \mathrm{~nm}$ gold with a single piece of 50 base (Sequence "B") DNA attached. ${ }^{3}$ The top view is an overhead-lit view, and the bottom is a transilluminated view of the same samples. Purplish or blue samples have aggregated, and are unusable for experiments. There was no difference in the preparation procedure for any of the samples, and the samples were all prepared at the same time. . . . . . . . . . . . . . . . .

3.10 Diagram of gel separation portion of the preparative tube gel apparatus.[14,

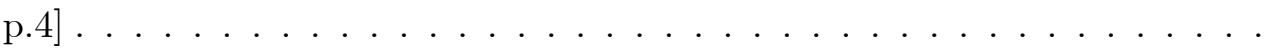

3.11 Diagram showing the procedure for making gold trimers starting with gold, thiolated DNA, and DNA trimers. . . . . . . . . . . . .

3.12 Representative branched DNA trimer preparative gel. The gel is $0.75 \mathrm{~mm}$ $10 \%$ denaturing PAGE, loaded at $20 \mu \mathrm{g}$ per lane, and run at $500 \mathrm{~V}$ and $50{ }^{\circ} \mathrm{C}$ until the loading dye (not shown) migrated a convenient distance to ensure separation of impurities. The central whitest band is the fluorescing, GelStarstained branched structure we desired. The remainder of the fluorescence in each lane is from impurities, such as $\mathrm{N}-1, \mathrm{~N}-2$, etc. strands, and from longer unintended synthesis products. We extracted only the desired full-length band from the gel. 
3.13 Comparison of three linear DNA components, same-sequence, by MALDI to the purified, branched DNA trimer. . . . . . . . . . . . .

$3.1420 \%$ denaturing PAGE gel showing the same trimer and standards as used in the MALDI in Figure 3.13. The branched 54 mer in Lane 5 plainly migrates much more slowly than the linear one in Lane 4. The first lane is loaded with a 10 base ladder standard. . . . . . . . . . . . . . . . .

$3.1512 \%$ native PAGE gel showing the successful hybridization of all arms of the DNA trimer we synthesized. The leftmost lane contains a $20 \mathrm{bp}$ ladder standard. The next two lanes contain the unthiolated 50 base strands that complement the three trimer arms. (Each trimer requires one "A'" strand, and two "B'" strands for complete hybridization.) The fourth lane contains the branched trimer species. The fifth lane contains the trimer plus one equivalent of $\mathrm{A}^{\prime}$, and the sixth lane contains the trimer plus two equivalents of B'. The last lane contains the trimer plus one equivalent of A' and two equivalents of B'. The uppermost retarded band in this lane is not present in any other lane, and it contains the completely hybridized trimer. The gel was run at $4{ }^{\circ} \mathrm{C}$ for about $5 \mathrm{hrs}$. at $160 \mathrm{~V}$ in $1 \mathrm{X}$ TBE. (Also shown as an example of a generic native gel in Figure 2.11.) . . . . . . . . . . .

$3.165: 1$ and 3:1 conjugate to trimer ratio reactions annealed at room temperature for five days. The reactions had a 2:1 mixture of $5 \mathrm{~nm}$ gold conjugates with Sequence A to those with Sequence H, and they were $0.84 \mu \mathrm{M}$ in gold, overall, with $50 \mathrm{mM} \mathrm{NaCl}$. The gel was $3 \%$ agarose run in $0.5 \mathrm{X}$ TBE until the bands achieved sufficient separation. The control lane contains both conjugates and salt, but no trimer. . . . . . . . . . . . . . . . . . .

3.17 TEM image of the sample extracted from the third, uppermost bands in the gel in Figure 3.16. . . . . . . . . . . . . . . . . .

3.18 Comparison of the percentage of gold particles in trimers for the gel-extracted trimer sample, and for a control of free gold particles. . . . . . . . . .

$3.193 \%$ agarose gel showing the results of a repeat of the experiment in Figure 3.16. This reaction was a 3:1 reaction of the conjugates to DNA trimer (this time trimer Sequence "J" and conjugates with Sequences "A" and "H"). Three bands are once again visible in the reaction lane, although they are fainter than in Figure 3.16. Unlike the five-day reaction in Figure 3.16, this reaction was only annealed overnight. . . . . . . . . . .

3.20 TEM image of topmost extracted band in gel shown in Figure 3.19. Trimer structures are not prevalent. . . . . . . . . . . . . . .

3.21 Results from a statistical analysis of the TEM data in Figure 3.20 from samples extracted from the gel in Figure 3.19. The two uppermost gel bands were analyzed. The percentage of trimers was small, and not much larger than were in the other band, not expected to contain them. . . . . . 
$3.223 \%$ agarose gel showing the results of hybridizing a DNA trimer with 5 and $10 \mathrm{~nm}$ gold nanoparticles. The $10 \mathrm{~nm}$ gold nanoparticles had a tendency to aggregate, shown by the presence of aggregates in the wells in lanes containing $10 \mathrm{~nm}$ gold. A band corresponding to a successful trimer reaction is not visible. This reaction was annealed overnight. . . . . . . . . . . . .

3.23 Diagram of possible species that might exist after annealing in an asymmetric trimer hybridization reaction. Number 1 labels the (most probable) fastest migrating species in a gel. Up to seven unique species may exist. At the time of this experiment it was unclear whether species 3 would migrate separately from 1 , and whether 4 would migrate separately from 2 . Would addition of a branched DNA strand 54 bases long perturb the 50 base gold conjugate enough to resolve a difference? We thought not, in reference to the results shown in Figure 3.16. If we had resolved that difference, we thought, there would have to be four bands showing in that gel, and there were only three.

3.24 TEM of the uppermost band in the gel in Figure 3.22. There is no clear indication of any preponderance of one type of species in this picture, or in the other pictures taken at the same time. . . . . . . . . . . .

3.25 Statistical analysis of TEM images taken of the topmost band in the gel shown in Figure 3.22. The $5 \mathrm{~nm}$ extracted gold control in this experiment was deposited at too high of a concentration, giving an abnormally high result for trimers in the free gold sample. The results, especially in light of the unconvincing TEM picture in Figure 3.24, are not conclusive. . . . . . . . .

3.26 Diagram of the target structure of a totally symmetric tetramer comprised of two DNA trimers and a single type of $5 \mathrm{~nm}$ gold conjugates. . . . . . . .

3.27 Diagram showing the seven conceptually unique species that could exist, post-reaction, in a symmetric tetramer hybridization mixture. Number one labels the fastest band in the mixture. . . . . . . . . . . . . .

3.28 Three $3 \%$ agarose gels showing the migration of three different reaction mixtures for a totally symmetric tetramer with $5 \mathrm{~nm}$ gold. The gel on the top-left has the most bands, with at least five, and possibly six visible. The gel on the top-right has fewer bands. The hybridization ratio of conjugates to trimer was lower in this case, so not enough active conjugates may have been present to drive the reaction to completion. (We expect some portion of the gold conjugates to be inactive, because the DNA may be non-covalently bound in some way to the gold surface.) The gel on the bottom (imaged in color) shows four bands in the reaction mixture. . . . . . . . . . . .

3.29 TEM looking for gold tetramer structures in the uppermost band in the top-right gel in Figure 3.28. The results are visually unconvincing. . . . . .

3.30 Statistics for all types of structures in each band for the top-right gel in Figure 3.28. The statistics do show an increased presence of tetramer in the fourth band, but it is not present in large quantities. "Other," which includes both single particles and larger aggregates, comprises a large portion of the structures on each TEM grid. . . . . . . . . . . . . . . 
3.31 Fourteen different species that may be present in a hybridized reaction mixture for an asymmetric tetramer. As with the other similar figures, number 1 labels the fastest band. . . . . . . . . . . . . . . . . .

$3.323 \%$ agarose gel showing preliminary results for an annealing reaction of an asymmetric tetramer. The bands in this gel were quite faint; probably the reaction would have benefited from the use of more concentrated conjugates. At least three bands are visible in the reaction mixture, although up to twelve would be expected (See Figure 3.31). If there are upper bands, they are not present in great enough quantity to see. The reaction mixture containing only one of the two trimers (the fourth lane) also has the same three bands, so the uppermost band we can see would be expected to contain two gold particles hybridized to a single DNA trimer. The reaction was annealed overnight. . . . . . . . . . . . . . . . .

$3.336 \%$ native PAGE gel showing annealing of all portions of the redesigned and synthesized $5^{\prime} \rightarrow 3^{\prime}$ tetramer structure. The lane second from the left contains the entire reaction mixture, but it does not seem to contain any bigger structure other than that of either half, separately. The reason for this problem is unclear. If the two halves had hybridized together, one would also expect to see an upper band in the lane where just the two DNA trimer parts were combined. This gel was run at $4{ }^{\circ} \mathrm{C}$ at $160 \mathrm{~V}$ for about 4.5 hours. The reactions were annealed at a $3 \mu \mathrm{M}$ concentration overnight with

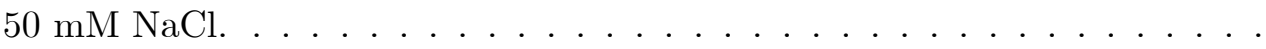

$3.343 \%$ agarose gels showing the result of adding $5 \mathrm{~nm}$ gold-DNA conjugates to separate DNA trimer components of a tetramer structure. The gel on the left contains one trimer half with gold, and the other gel contains the complementary trimer with its hybridized gold conjugates. These reactions were annealed for one day. . . . . . . . . . . . . . . . . .

$3.353 \%$ agarose gel, run by Sarah Goh, showing the behavior of trimer and tetramer structures with only some components added. Pictures indicate the possible most-hybridized structure for those lanes. In lanes labeled underneath with numbers, that value is the ratio of conjugate to trimer. Only one type of conjugate has been added in those lanes. Lanes 8 through 11 should show comparable results to Figure 3.34, but they contain one less band. Lane 7 has all the components to make complete trimers, but hybridization ratio was only $1: 1$. Lane 12 has all the components to make a complete tetramer in a $2: 1$ stoichiometric ratio. These reactions were annealed overnight. . . .

4.1 Tile "A," one of two tiles in a lattice system reported by Prof. Seeman's group at NYU. [2] . . . . . . . . . . . . . . . . . .

4.2 Cartoon of the structure of a four-way DNA junction called a "Holliday Junction." $[15] \ldots \ldots \ldots \ldots \ldots \ldots$

4.3 Tile "B" complement to "A" in Figure 4.1. Both Tiles A and B have four "sticky ends" apiece. Tile B has two hairpins. Because of the helix turns not pictured in this cartoon, one of the hairpins sticks up from the surface of the tile, and the other sticks down in the opposite direction. [2] . . . . . 107 
4.4 Tiles $\mathrm{A}$ and $\mathrm{B}$ have sequences designed to complement each other at their corners. Self-assembly leads to two-dimensional DNA array. The array has a periodicity of $25 \mathrm{~nm}$ from the stripes formed by the hairpin bumps coming from the top of Tile B. $[2] \ldots \ldots \ldots \ldots$

4.5 Tile components for a four-tile lattice system. Tiles B and D have hairpins that stick up above the resulting lattice fabric. Tile B's hairpins are nicked so the hairpin strand can be included or not as wished.[3] . . . . . . . . .

4.6 Figure showing rectangular tiles labeled with nanocrystals, combined with an set of unlabeled tiles to form a nanocrystal array on top of a two-dimensional lattice structure. . . . . . . . . . . . . . . . . .

4.7 A scheme of the experiment plan for making ordered arrays of nanocrystals using DNA lattices as scaffolding. . . . . . . . . . . . . . .

4.8 Either 10 or $15 \%$ denaturing PAGE gels purifying various strands out of the 24 necessary to prepare a four-tile lattice system. The identity of the strand in any particular gel in the image is not of interest here. The point is to merely give an overview of how such a set of gels would appear. This would not be nearly a sufficient number of gels to prepare all of the lattice strands. Usually, we needed 4 or more gels of any particular strand to yield enough product to do experiments. The result was that 100 or more gels were necessary to produce enough DNA to make a set of lattices. . . . . . .

$4.912 \%$ native PAGE gel showing the formation of Tile A. The yellow and green strands are each 26 bases long, and the red and blue strands are 48 bases long. The highest band in the second lane from the right, with all four strand components, contains complete double-crossover tiles. This gel was run in $1 \mathrm{X}$ TAE with $12.5 \mathrm{mM}$ magnesium acetate and $3 \mathrm{mM}$ sodium acetate, recirculated at $300 \mathrm{ml} / \mathrm{min}$. at $4 \mathrm{~W}$ constant power for $15.5 \mathrm{hrs}$. The tile anneal was $1.9 \mu \mathrm{M}$ in all strands, and in a magnesium-containing buffer. . .

$4.108 \%$ native PAGE gel showing the formation of Tile B. The red and blue strands are each 26 bases long, and the green and yellow strands, which have hairpins, are 70 bases. This tiles components gave complicated, highly retarded structures as soon as the complementary 70 base strands were both present, and the complete tile formation was in the brightest band on the right-hand lane, not the upper, lighter bands. We do not know what structures were in those uppermost bands. This gel was run in 1X TAE with $12.5 \mathrm{mM}$ magnesium acetate and $3 \mathrm{mM}$ sodium acetate, recirculated at 300 $\mathrm{ml} / \mathrm{min}$., for $3 \mathrm{hrs}$. at $80 \mathrm{~V}$ and $7 \mathrm{hrs}$. at $100 \mathrm{~V}$. The tile was annealed at a $1 \mu \mathrm{M}$ concentration in magnesium-containing buffer. . . . . . . . . .

4.11 Schematic showing the process for making and modifying DNA lattices with gold nanocrystals. The thermal profile on the top right of the figure is actual data of the temperature versus time for an annealed reaction floated in $2 \mathrm{~L}$ of boiled water in a beaker inside a styrofoam box. . . . . . . . . . . . . . 
4.12 Diagram of electrodialyzer setup supplied by Harvard Apparatus (Amika). A teflon chamber with screw-on teflon caps created a sample chamber. The teflon caps were hollow, as shown in the picture. They affixed membranes (of a type and size chosen by the user) on both sides of the chamber, allowing the internal sample to make electrophoretic contact with the surrounding buffer. The portion of the sample smaller than the membrane's MWCO leaves the internal chamber after only 5 or 10 minutes. Additional chambers could be screwed to the first to trap samples of different sizes behind different membranes, if desired.[16] . . . . . . . . . . . . .

4.13 Diagram showing how an atomic force microscope works. Very roughly, it works similarly to a record player, but on a much smaller scale. A probe with a tip with a radius of less than $10 \mathrm{~nm}$ fabricated from a silicon or silicon nitride wafer is scanned across the sample surface. A piezocrystal controls the vertical and horizontal position of the tip relative to the surface. The back of the probe is reflective, and a laser bounces off of the back of the probe onto a split photodiode detector. The position of the laser light reflection on the photodiode tells the detector electronics the angle of the bend of the cantilever, which is affected by the surface features. This information allows the controller electronics to adjust the height of the probe, using the piezocrystal, to reflect the surface topography, and the computer records the height information as it rasters across the surface to create a computerized topographic image. A variation on this process vibrates the probe cantilever near its resonant frequency, and the detector records dampening of its oscillation, allowing the controller to adjust height with that information to keep the amplitude constant. The advantage of this method, (called "tapping mode"), is that the tip does not, then, directly drag across the surface. Dragging can damage a softer sample. . . . . . . . . . . . . . .

$4.145 \mu \mathrm{m}$ AFM scan of a sample of pPIC9 plasmid. This plasmid has 8023 base pairs, and a molecular weight of $5.2 \times 10^{6}$ Daltons. To prepare this sample, we treated a freshly cleaved mica substrate overnight with $33 \mathrm{mM} \mathrm{Mg}(\mathrm{OAc})_{2}$, sonicated it in distilled water for 20 minutes, dried it with compressed air, glow-discharged it for 40 seconds under 100 mTorr of argon, and then inverted it onto 1 drop of $190 \mathrm{nM}(990 \mathrm{ug} / \mathrm{mL})$ plasmid sample in $10 \mathrm{mM}$ Tris- $\mathrm{HCl}$ buffer, pH 8 containing $1 \mathrm{mM}$ EDTA. We rinsed the sample with distilled water, and then dried it with compressed air. We imaged the sample in contact mode with a Digital Instruments Bioscope under n-propanol with a silicon nitride tip $(\mathrm{k}=0.32 \mathrm{~N} / \mathrm{m}) \ldots \ldots \ldots \ldots$

4.15 Two images of pUC19 plasmid $\left(3500 \mathrm{bp}\right.$, MW $\left.2.28 \times 10^{6}\right)$. Both were collected on a Digital Instruments Multimode IIIa AFM in tapping mode. We deposited the sample on freshly cleaved mica at the same time we imaged in a $1 \frac{\mu g}{m l}$ solution of $10 \mathrm{mM}$ HEPES buffer with $5 \mathrm{mM} \mathrm{NiCl}_{2}$. The AFM tip was made of silicon nitride, with a spring constant of $0.32 \mathrm{~N} / \mathrm{m}$ and radius less than $20 \mathrm{~nm} . \ldots \ldots \ldots \ldots \ldots \ldots \ldots$ 
4.16 An AFM image (published by Winfree, et. al. in 1998) of lattices made of a two tile, 10 strand, system. The scale bar is $300 \mathrm{~nm}$, and the lattice has stripes created by hairpins every $32 \mathrm{~nm} .[2] \ldots \ldots$. . . . . . . . . .

4.17 Good-quality image of four-tile system lattices with a hairpin every $64 \mathrm{~nm}$. The image on the left is a topographic image. The image on the right is a phase image. The lattices have grown quite large, and are folded back on themselves in places. . . . . . . . . . . . . . . . .

4.18 Image of a section of lattices from Figure 4.17 used to determine the periodic spacing of the ridges. The ridges were designed to be $64 \mathrm{~nm}$ apart. The measured result of $63.7 \mathrm{~nm}$ is very reasonably close to this value. . . . . . .

4.19 A $10 \mu \mathrm{m}$ AFM scan and a $20 \mu \mathrm{m}$ AFM scan of four tile DNA lattices. . . .

4.20 A $7.5 \mu \mathrm{m}$ AFM scan and a $10 \mu \mathrm{m}$ AFM scan of four tile DNA lattices. . . .

4.21 A $6.8 \mu \mathrm{m}$ AFM scan and a $30 \mu \mathrm{m}$ AFM scan of four tile DNA lattices. For part of the $30 \mu \mathrm{m}$ scan the probe temporarily lost contact with the sample, but at the necessarily slow $0.79 \mathrm{~Hz}$ scan rate, that problem is not surprising, and was hard to prevent. Our capacity to do a $30 \mu \mathrm{m}$ scan of this kind of sample was actually quite good. . . . . . . . . . . . . .

$4.22 \mathrm{~A} 3.6 \mu \mathrm{m}$ and a $2.0 \mu \mathrm{m}$ AFM scan of two lattice samples. In the lower image, the rows of DNA hairpins on Tile D are clearly visible, along with the available sites for hairpin or gold-conjugate addition on Tile B, which make a fainter line in between the Tile D ridges. . . . . . . . . . . . .

$4.23 \mathrm{~A} 15 \mu \mathrm{m}$ scan and a $980 \mathrm{~nm}$ lattice scan. It was hard to get good-quality images at a small scan size (like the one on the bottom) because the DNA seemed to be dragged around more by the AFM tip. This image was exceptionally good for its size. . . . . . . . . . . . . . . .

4.24 A $30 \mu \mathrm{m}$ scan and a $3.43 \mu \mathrm{m}$ scan. These images have some noise in the phase image, but the topography mode gave a nice image. The phase images on the right were more typical of a phase image on a good, but not exceptional day. Better phase images were exceptional. The top image, once again, has a region where the AFM tip lost contact with the sample, but given the large scan size, that problem was expected. . . . . . . . . . . .

$4.2515 \mu \mathrm{m}$ and a $20 \mu \mathrm{m}$ scans. . . . . . . . . . . . . . . . . .

4.26 A scan showing lattices surrounded by a large amount of unincorporated DNA. This lattice reaction was not very successful, and there are many smaller structures on the substrate. . . . . . . . . . . . . . . .

4.27 Two images of separate failed lattice batches. The DNA has deposited according to the drying pattern of the solution. . . . . . . . . . .

4.28 Two more images of failed lattice batches. It seems that perhaps some small structures have formed in the sample in the bottom image. . . . . . . . .

4.29 Yet two more failed lattice batches. This kind of image was common in unformed lattice batches. . . . . . . . . . . . . . . . . . .

4.30 Two last failed lattice batches. DNA strands and small structures are visible in the bottom image. The bottom image can be compared to the image in Figure 4.26, where a few lattices still formed amongst a similar mess. . . . . 
4.31 Plot showing whether lattices formed in any particular experiment versus time of year, and time left annealing. The time of year data could give clues to whether the DNA strands had degraded over time. Many experiments were conducted simultaneously, so every dot does not necessarily correspond to a single experiment. "1st Attempt," "2nd Attempt," etc. correspond to the first, second, etc. attempts to look for lattices in the sample under AFM. Sometimes lattices would not form before the initial check, but after the passage of more time, would form. . . . . . . . . . . . . . .

4.32 Before and after pictures for a lattice sample with $5 \mathrm{~nm}$ gold conjugates added. The gold binds non-specifically everywhere on the surface of the lattices, instead of binding specifically to the designed and available hairpin binding sites. . . . . . . . . . . . . . . . . . 146

4.33 Before and after pictures of a sample of electrodialyzed lattices. . . . . . . 147

4.34 The top image, from Xiao, et.al. shows lattices before addition of gold, and the bottom shows lattices after gold addition. $[17] \ldots \ldots$. . . . . . . .

4.35 A comparison of the backbone structures of PNA and DNA. The figure shows PNA on the left, and DNA on the right. . . . . . . . . . . . . . 149

A.1 5' and 3' ends labeled on a 2'-deoxyribonucleoside . . . . . . . . . . . 165

A.2 Acetic anhydride molecule. . . . . . . . . . . . . . . . . 165

A.3 2'-Deoxyadenosine . . . . . . . . . . . . . . . 166

A.4 Amine group. . . . . . . . . . . . . . . . 166

A.5 Figure showing the sugar backbone of the DNA strand. . . . . . . . . 167

A.6 2'-Deoxycytidine . . . . . . . . . . . . . . . . 168

A.7 Dimethoxytrityl group that protects an hydroxyl group as an ether. . . . . 169

A.8 2'-Deoxyguanosine . . . . . . . . . . . . . . . . . 169

A.9 Figure showing the phosphodiester linkage in a DNA strand backbone. . . . 171

A.10 Figure comparing DNA and RNA nucleosides. The RNA one has a 2' hydroxyl group. . . . . . . . . . . . . . . . . . . . 172

A.11 2'-Deoxythymidine . . . . . . . . . . . . . . . . . . 174 


\section{List of Tables}

2.1 Extinction Coefficients for 5 and $10 \mathrm{~nm}$ Au nanoparticles at $520 \mathrm{~nm}$. . . 


\section{Acknowledgments}

I want to start by thanking my parents for all their years of support, and for sparking my curiosity about the world. I would also like to thank my grandparents, who offered encouragement and unwavering support.

I thank Paul Alivisatos for allowing me to work in his research group. I am grateful for his support both throughout graduate school and as I begin my career. I have learned a lot about how to do scientific research from him, and I have appreciated the tremendous, awe-inspiring opportunity I have had to work in the cutting-edge scientific "playground" he has built.

The research work in this disseration was a collaborative effort more often than not, and I want to thank Sarah Goh, who worked jointly with me on the trimer project. I also would like to thank Colin Loweth, who helped me generously at the start of the lattice project. I thank Daniele Gerion, who is both a competent scientist and a great person, and who did the main work for the semiconductor silanization project. I also thank Christine Micheel, who cheerfully trained me to work with the gold nanoparticles, and performed TEM and statistical analyses on the results for the trials reported here. All of these people were positive examples to me, were generous with their time and helpful advice, and were fun to work with.

Although the lattice project did not turn out to work in the time allotted, I would still like to thank Prof. Ned Seeman for his generous hospitality at New York University during the three and half months I spent in his lab. I thank Furong Liu, also, for training me to make DNA lattices. The rest of the Seeman lab members were wonderfully hospitable 
while I was there, and I enjoyed many great conversations about both science and the rest of life.

Some forms of support in the Alivisatos Group or the rest of the Chemistry Department did not include pushing beakers, but were still vital to a positive graduate school experience. In addition to the people I mentioned above, many people have helped me throughout the last six and a half years, and through my forgetfulness, I am sure to neglect to name an important person. I hope, if you are that person, you will forgive me, and know that I am still grateful.

(I'm starting to sound like an Oscar winner past the thirty-second time limit, but I really am grateful to many people!)

Some people from the earliest time I spent working for Paul were essential to helping me begin. CJ Lee has always been caring and generous with her time, even going as far as fixing one of the figures in this dissertation. Keren Jacobs, Hongkun Park, and Xiaogang Peng have been very supportive, especially in the early period of my work in this lab. Kim Hamad-Schifferli and Marcel Bruchez were also friendly and helpful with their advice during that early period. I thank Liang-shi Li specifically for a very dedicated week of help with Statistical Mechanics in advance of that class's final exam. I also thank Saskia Hoffer and Jessica Rogers for their good company in classes.

For help, support, and companionship in the most recent years, I thank Ben Boussert, Deborah Aruguete, Josh Wittenberg, Aihua Fu, Emory Chan, Carsten Soennichsen, Dong Hee Son, Can Erdonmez along with all of the other Alivisatos group members. I has been a great experience working in this lab with all of these neat people. I wish the 
members of the Alivisatos group the best of luck in the future.

I could not have graduated without the support of my committee members, Professors Herb Strauss and Arun Majumdar. Thank you to both of them.

I thank Sunny Nguyen, Elisabeth Christensen and Ted Lam for years of happy friendship while we were in the San Francisco Bay Area together. I am grateful for these particular friends and for the others I have had the privilege of knowing throughout the years.

I am grateful to the members of the cooperative house I have lived in for three years. My graduate school experience wouldn't have been the same without the opportunity to meet and enjoy the company of so many interesting people. I wouldn't trade all of that fun for my own private apartment!

Graduate school has been mostly fun, at times an exceptionally character-building challenge, and always hard work, but I am very happy I chose to study for a Ph.D. in Chemistry at UC Berkeley. 


\section{Chapter 1}

\section{Motivation and Background}

Enthusiasts from chemistry, physics, biology, materials science, and engineering are uniting their skills to explore the burgeoning field of nanotechnology. The study of "nano" (a prefix for lengths one-millionth the size of of a millimeter) represents the culmination of years of study of both the world we can see and the world of atoms. This field seeks to control the interface between very tiny things and the things we can see and hold. The goal is to introduce functional design to structures that have at least one dimension in that "nanometer" size range between the unfathomably small molecules and atoms that traditional chemists manipulate, and the larger world that we can see (or at least see in a microscope) of materials, physics, and biology. Scientific American recently published a set of articles describing the enthusiasm and excitement behind this developing field in which one article states:

The nanoworld is a weird borderland between the realm of individual atoms and molecules (where quantum mechanics rules) and the macroworld (where the bulk properties of materials emerge from the collective behavior of trillions of atoms, whether that material is a steel beam or the cream filling in an Oreo). Scientific American, September 2001, Gary Stix[18] 
Nanotechnology is really technology at an interface. It is a nexus of disciplines for the scientific generalist. The nanotech scientist treads the pathways between the departments for the traditional disciplines of chemistry, physics, biology, and engineering, and the resulting mixing has led to new ideas that combine the interests and training of people in all of these fields. In this field, it is not surprising to see physicists working with cells, biologists concerned with materials, and chemists working with electronics. In some cases, we have looked back at even older research ideas and have seen them in new light, relabeling and converting them to nanotech-oriented purposes.

Much of the interest in nanotechnology seeks to understand and exploit the unique attributes of nanometer-scale things. These kinds of structures are too big to be described by the traditional chemistry that works with synthetic techniques to build bigger and bigger things one atom or component at a time. These structures are not so big, however, that the unusual physics of quantum mechanics that completely governs the properties of smaller molecularly-sized things fails to influence the properties and interactions of the only somewhat slightly bigger nanoscale objects. Nanotechnologists need to understand the significance of quantum-mechanical effects if they want to build functional structures on that scale, and with that understanding, they may come up with new ways to make use of the special properties of small-scale devices.

My work in this dissertation focused on the specific research area of nanobiotechnology. Living things are a model system for making nanotechnology work. Many of the important processes in biology harness the interactions between nanometer-sized things. Proteins, membranes, DNA, RNA, viruses all perform nanoassembly in a natural 
and effortless way. Our interests have combined portions of the fields of biology, chemistry, and materials science. This endeavor begins at the top of the cusp between biology and materials science, and as such, has the potential to lean either direction. We could either lean toward doing biology research, using our materials chemistry knowledge to invent or discover new methods to solve biological problems, or we could lean toward doing materials research, and use biological structures to enable production of new materials. The work described here is more the latter, but work in this research group (and in others) currently progresses toward both goals.

Our work centered on developing pieces of a nanotechnology toolkit. We have wanted to create a sort of stockpile of nano-sized building materials. If we could have on-hand a toolkit or library of techniques that allow us to fabricate nanostructures, we can design a nanostructure to suit our purposes, and then select the most appropriate pieces of our toolkit to accomplish that task. In order to make a new structure, we do not have to develop a whole new toolkit. Instead, the toolkit exists, independent of design and function, and we engineer the flexibility we will need into it from the beginning.

The materials we want to arrange in large part govern our choice of toolkit components. It must be possible to tailor the toolkit to produce a variety of structures, and these structures must be similar in size to the objects we want to control with the toolkit. Thus, since we want to have nanometer-scale control, the toolkit must assemble nanometer-sized structures.

Our research group has previously developed familiarity and expertise in making small crystalline particles called "nanocrystals" or "nanoparticles" about one to ten 
nanometers big. We desire to pattern them in logical and controlled ways so we can control and study their optical, electrical and chemical interactions with each other, and with other kinds of structures and materials.

The remainder of the first chapter of this dissertation discusses the types of nanoparticles we worked with and the reasons we want to make arrangements of them. It also describes the biological molecule, DNA, and why we chose to use DNA to make our nanoparticle arrangements. The second chapter discusses how we prepared and characterized our samples. Chapter 3 details one set of experiments using branched DNA trimers to arrange nanoparticles, and Chapter 4 describes our progress toward making ordered arrays of nanoparticles using DNA lattices.

\section{$1.1 \quad$ Nanocrystals}

\subsubsection{What they are}

The nanocrystals we used for our experiments are small crystals of hundreds to thousands of atoms. Their size is in between smaller molecules that contain only a few atoms, and larger pieces of material that exhibit the properties of bulk. Crystals of this intermediate size can exhibit strongly size-dependent properties.[19]

Some structures of this intermediate nanometer size are not reliably crystalline,

or at least a single crystal. These amorphous or polycrystalline structures, can still be interesting to use for their own structural, chemical, or physical properties, and together with nanocrystals, are termed "nanoparticles. They can serve as model systems for assembly of larger structures comprised of multiple nanoparticles, or they can be useful for their 
properties by themselves.

We used two categories of nanoparticles in our research reported here: gold nanocrystals and semiconductor nanocrystals. The semiconductor nanocrystals were made of crystalline cores of cadmium selenide, surrounded by shells of zinc sulfide. For historical reasons, most of the previous literature on gold nanocrystals refers to them as "nanoparticles,"(for example, see:[12][20][21][22][23][24][25][26][27][28][29][30][31]) and this document follows that precedent.

\subsubsection{Why they are interesting}

The properties of nanocrystals depend on the materials that comprise them. Nanocrystals made out of metals or insulating materials tend to have properties similar to that of bulk material, but their small size amplifies the effect of their surface on their behavior. Semiconductor nanocrystals have more size-dependent properties because the spacing between their valence and conduction bands changes as the size of the nanocrystal changes, in addition to their surface-to-volume ratio. It is possible to chemically modify and electrically passivate the surfaces of the nanocrystals to change their behavior, and to enable us to incorporate them into larger structures that would take advantage of their special optical, electronic, magnetic, or structural properties.[19]

\subsubsection{Types of nanocrystals we used}

\section{Gold Nanoparticles}

The studies described here make extensive use of gold nanoparticles. Gold nanoparticles have three advantages for use in nanostructures: 
1. The particles are easy to make in controlled sizes, and with a small size distribution. As a result, and as a result of their usefulness as an imaging agent in transmission electron microscopy (TEM), it is possible to purchase a library of particles in useful sizes from at least one commercial source.[32]

2. The particles can be synthesized in water, and thus are easily water-soluble.

3. The gold nanoparticles can be easily conjugated to biomolecules such as DNA through a thiol bond. The thiol bond is a strong covalent bond, and does not lead to noticeable amounts of exchange of the conjugated material after addition. ${ }^{1}$

\section{Semiconductor Nanocrystals}

We used gold nanoparticles in most of our DNA-based nanoconstruction experiments, but we also worked toward incorporating nanocrystals made out of the semiconductor, cadmium selenide, into our nanostructures. The preparation of the cadmium selenide nanocrystals that are water soluble is much more challenging than making water-soluble gold, though. Our strategy was that it would be easiest to work out the nanofabrication using the easier-to-prepare gold nanoparticles in the initial stages, and then, once the nanofabrication technology had been at least partially developed, add in the semiconductor nanocrystals.

The useful feature of the cadmium selenide nanocrystals is that they fluoresce in a narrow band adjustable over a range between 470 and $644 \mathrm{~nm}$, depending on their size within a range between 1.5 to $8 \mathrm{~nm}$.[4][7] Figure 1.1 shows the fluorescence of a selection

\footnotetext{
${ }^{1}$ Colin Loweth, unpublished data
} 


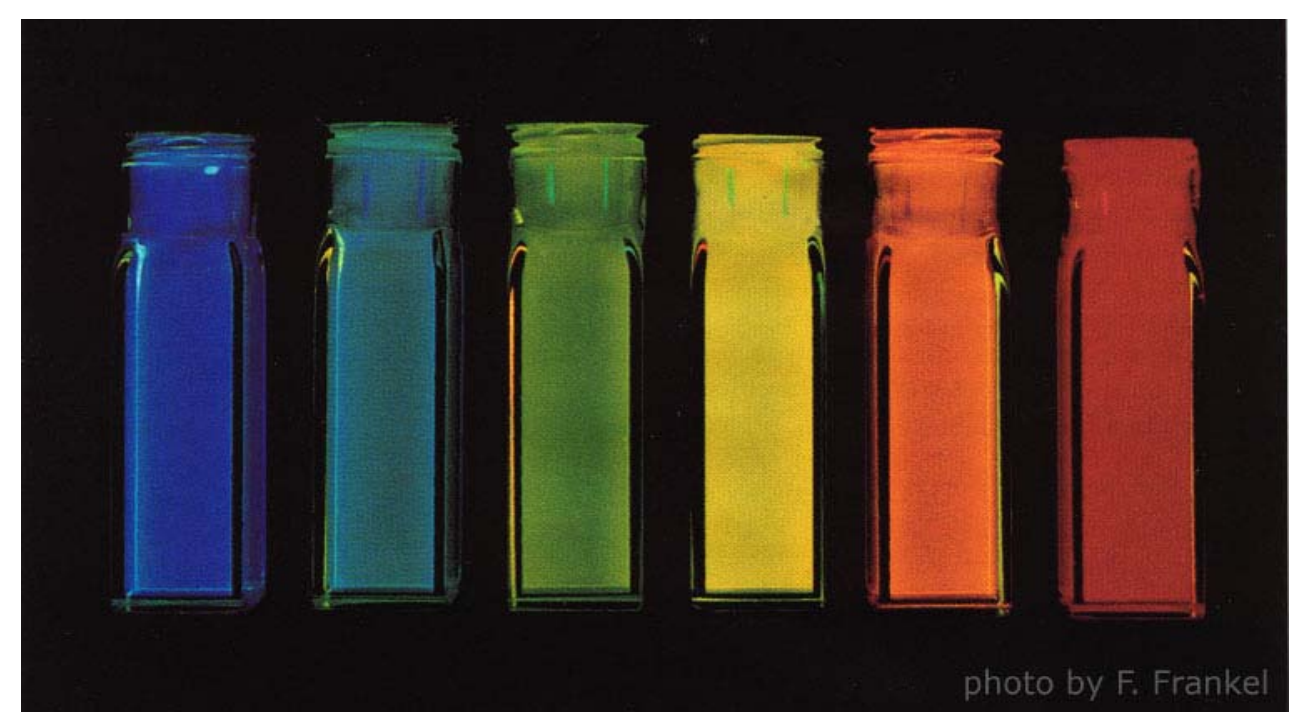

Figure 1.1: This figure from Dabbousi, et. al. shows a selection of six different sizes of $\mathrm{CdSe} / \mathrm{ZnS}$ core/shell nanocrystals under excitation with a $365 \mathrm{~nm}$ source luminescing in a range of wavelengths between 470 and $620 \mathrm{~nm}$, left to right.[4]

of different sizes of these cadmium selenide nanocrystals, covered with an outer shell of zinc sulfide. Unlike organic fluorescent dyes, these nanocrystals absorb over a wide spectrum, allowing their fluorescence in a variety of colors to be excited by one single source. Also, the technique for encasing the outer surface with the zinc sulfide material that has a wider band gap makes the quantum yield for the fluorescent semiconductor nanocrystals increase 50-70\%.[33][4][7] We can encoat these nanocrystal core/shells with a water-soluble siloxane layer in order to incorporate them into biologically-based systems and structures. [34][7][35]

\subsection{Reasons for Making Nanocrystal Arrangements}

We would like to have specific control over the relative and absolute positions of different types of nanocrystals to study their interactions. For example, based on previous studies of bulk material and theoretical predictions, we expect close proximity (inside of 
a few nanometers) of gold nanoparticles to semiconductor nanocrystals to enhance the fluorescence of the semiconductor nanocrystals.[36][37] Aihua Fu in our research group is currently studying the fluorescence enhancement of semiconductor nanocrystals surrounded by discrete numbers of gold particles attached by DNA strands in a method related to the work described in this dissertation.

Carsten Soennichsen is performing another study where it is useful to control the relative spacing between two or more metal nanoparticles. Illumination of metal nanoparticles with light causes the conduction electrons in the nanoparticles to oscillate at their "plasmon" frequency. This oscillation causes the light having that frequency to scatter or to be absorbed. The charge separation in the induced dipole increases when two metal nanoparticles are close enough to interact, which red-shifts the resulting plasmon to a degree that varies with distance He can see the color change resulting from discrete groupings of particles by looking at scattered light in a darkfield microscope, and we can use DNA to control the type and placement of the particle groupings.

A third example of an application where this kind of DNA-templated control for nanoparticles would be useful is in creating nanoparticle chain arrays such as that described by Brongersma, et. al.[38] and Maier, et. al.[39]. In their work, they have shown that closely-spaced chains of metal particles with a diameter smaller than the wavelength of incident light can act as wave guides, transferring electromagnetic energy by coupling plasmon excitations in the nearby particles. DNA could be useful for templating these types of nanoparticle chains. 


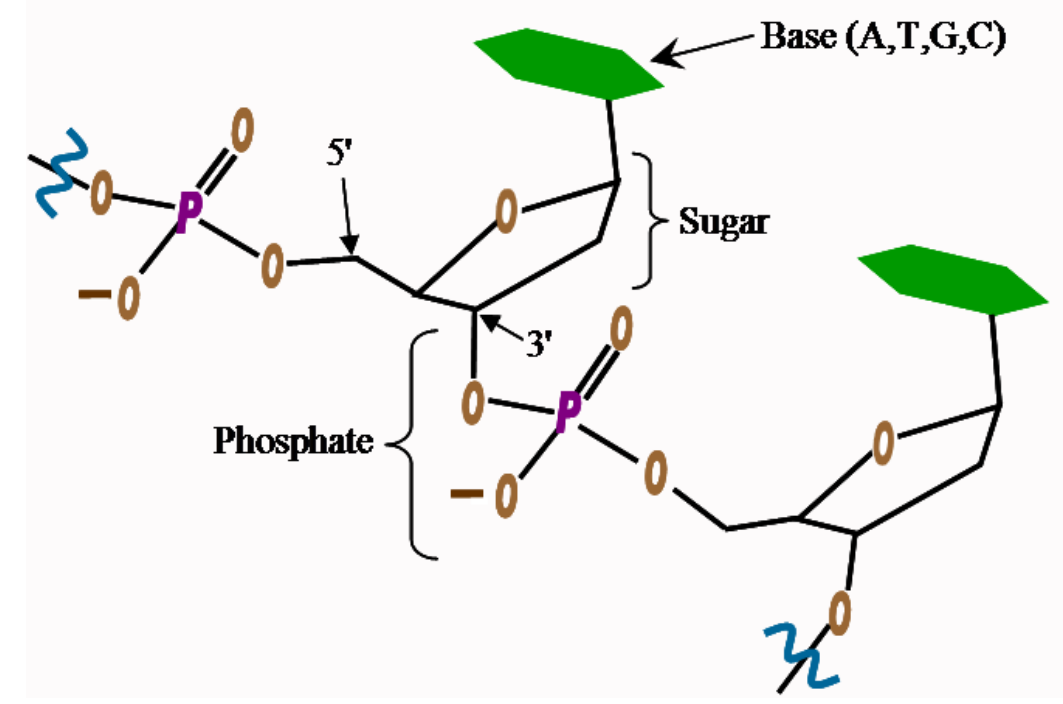

Figure 1.2: 2'-Deoxyribose joined by phosphodiester linkages forms a polymer of sugars to make DNA.

\subsection{DNA as a Template Material for Nanocrystals}

\subsubsection{What DNA is, and what it can do}

DNA, 2'-deoxyribonucleic acid, is a polymer comprised of units of a pentose sugar (2'-deoxyribose), each labeled with one of four bases, adenine, guanine, cytosine, or thymine. It differs from RNA (ribonucleic acid) through the lack of a hydroxyl group in the sugar's 2' position, (and through the presence of the base thymine instead of uracil). Successive sugars are linked by a phosphodiester bond, as shown (without the sugar hydroxyls) in Figure 1.2. The strands have two different ends, each labeled by the last carbon on the sugar, either " $5^{\prime \prime}$ or " $3^{\prime} . "$

DNA's unique properties largely result from the properties of its four bases. The bases give the DNA its configuration, or sequence, and encode all of the information the sequence contains. The four bases fall into two types: purines and pyrimidines. The 


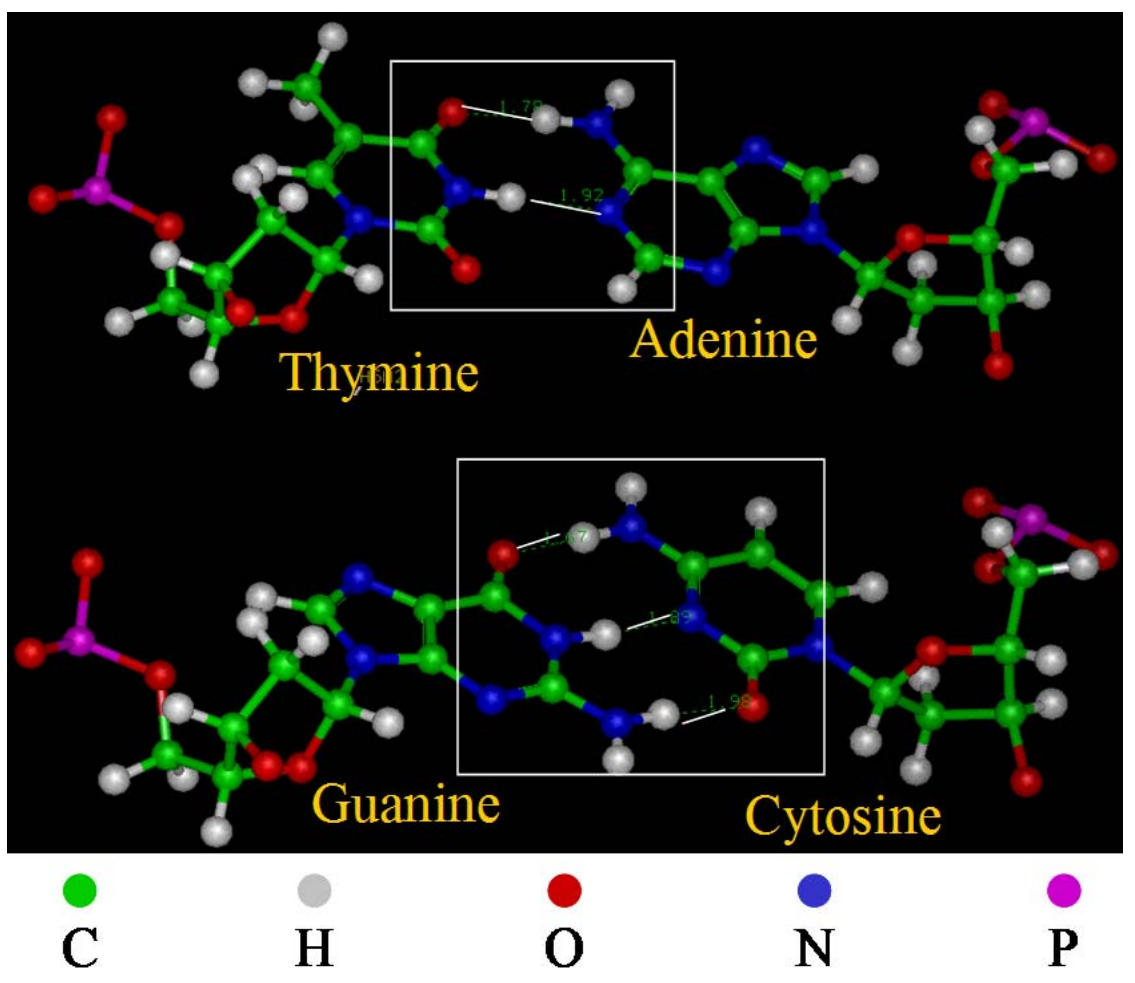

Figure 1.3: Model of Watson-Crick base pairs in DNA. Juergen Suehnel, Image Library of Macromolecules, Jena/Germany.[5]

pyrimidines, cytosine and thymine, are six-membered heterocyclic bases containing two nitrogens. The purines, adenine and guanine, have two conjoined heterocyclic rings.[40] The substituents on all four bases enable hydrogen bonding. Watson and Crick discovered in 1953 that, in natural conditions, adenine (A) specifically base-pairs with thymine (T) using two hydrogen bonds, and that guanine base-pairs with cytosine $(\mathrm{C})$ with three hydrogen bonds.[41] Figure 1.3 shows these base pairs. The two specific pairs of bases form the strands of DNA into a double helix with a fixed width of $2 \mathrm{~nm}$, as shown in Figure 1.4. The helix completes a turn after a progression of 10 base-pairs (or a distance of $3.4 \mathrm{~nm}$ ).[42] Watson and Crick's discovery of the double helix enabled them to win the Nobel 


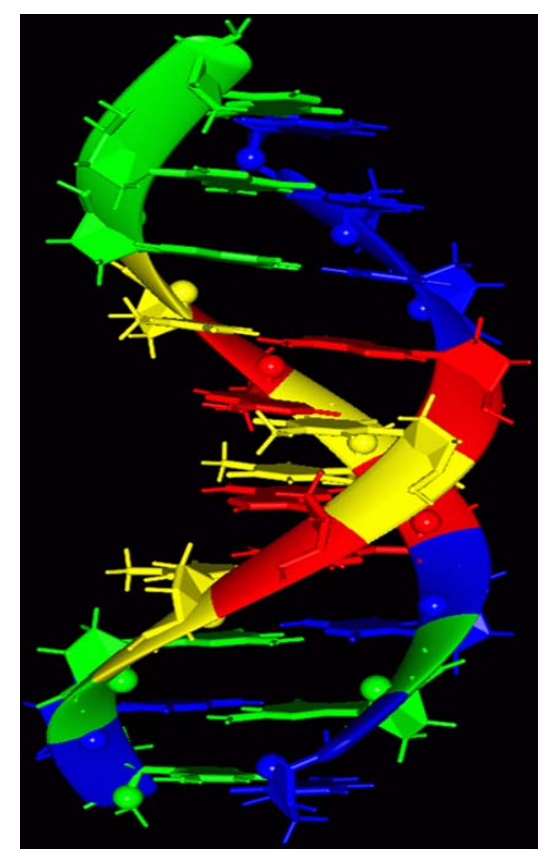

Figure 1.4: Two strands of DNA with complementary base sequences hydrogen-bond to form a double helix. Juergen Suehnel, Image Library of Macromolecules, Jena/Germany.[5]

Prize in 1962. The reason for all of the excitement, of course, was the special role that DNA plays for life. Cells and viruses contain DNA (although they package it in different forms) to encode information about life. This information is contained in the sequence of bases that govern the binding of DNA to itself, to RNA, and to proteins. The specific bases that pair together in DNA allow it to replicate, recombine, and turn that information into instructions for making proteins.

The key point of this digression into biology is to emphasize that, as a polymer, DNA exists to encode information. Nature takes that information and creates life. It is possible, however, to deviate from the natural life-encoding purpose of DNA, and to use this polymeric molecule for its unique structural properties. We can encode information about structure or identity into the DNA molecule, by controlling the sequence of its bases, 
and use it for whatever purpose we would most like.

\subsubsection{DNA in nanotechnology}

The physical size and information-encoding properties of DNA make it useful in nanotechnology experiments. The DNA double helix, with its diameter of $2 \mathrm{~nm}$ and its helix repeat unit of $10 \mathrm{~nm}$, has convenient properties in the nano-length scale. Experiments controlling the position, placement, and relations of different nanosized items find that a molecule like DNA, with its nanoscale-length properties, can be a useful scaffolding or template.[43][44][45][27][28][22][12][46][1][17][47]

The DNA that we used in the experiments described here differs from natural DNA in four ways:

1. The information encoded in its base sequence only provides instructions for selfassembly so it will make a scaffolding that arranges nanocrystals or nanoparticles. At this current stage of research, it does not encode instructions for the synthesis of other things, as it would do in life.

2. Its sequence is entirely unnatural, and its source was no living thing. Nature synthesizes DNA using DNA polymerase, starting at the 5' end. The DNA we use is chemically synthesized. It is synthesized in the opposite direction from nature. Chemical synthesis, now conveniently automated, synthesizes DNA from the 3' end.

3. Its length is short. Naturally occurring DNA is long. For instance, the DNA in the bacterium E. coli is about $4.5 \times 10^{6}$ base pairs long, and human cells contain $4.0 \times 10^{9}$ base pairs. [48] Automated chemical synthesis is limited by the fact that the steps 
of synthesis are sequential, and errors from previous steps are inherited. While the process of chemical synthesis has been improved so that individual yields for adding each base to a strand are better than 99\%,[49] synthesizing strands longer than 100 bases requires rigorous attention to the maintenance of the synthesizer and choice of support and reagents. Strands longer than 150 bases require dedicated expertise. Obviously, these small numbers for the length of the DNA strands we can synthesize chemically come nowhere near the size of the DNA length for humans, or even E. coli.

4. Most natural DNA exists in a simple double helix form. The DNA we have used here has been in complicated, non-natural, branched structures, and it contains nonnatural thiol or amino labels that will attach to other things. The branches have formed in two ways. One way was to have the DNA sequence of bases require that a given DNA strand first hydrogen-bond with a second DNA strand to form a helix, and then, in a region further on in the structure, it exchanged strands to participate in a different helix with a third DNA strand complement. This kind of structure does occur naturally during recombination, as a "Holliday Junction." The other way involved the chemical synthesis of a covalent branch in the DNA sugar backbone. A single DNA strand thus covalently splits into two. Both kinds of branching enable our DNA structures to attain new kinds of complexity, and the synthetic addition of a binding ligand into the strand allows the DNA to interact with substrate surfaces or nanocrystals. 


\subsection{Benefits of Using DNA to Organize Nanocrystals}

The reasons we chose DNA as the molecule to template our nanocrystal arrangements were that we could rely on it to provide us with a method to make the structures self-assemble, that we could program it, that it would enable us to control distances between particles, and that we could target it to certain locations on a surface. These properties were essential, and are described in detail below.

\subsubsection{Self-assembly}

Biology excels at producing nanostructured materials. It produces small, defined aggregates that have properties that cause themselves to spontaneously move into largerscaled structures that have both function and meaning. This process moves throughout all of life to produce structures with progressively higher scales of order, from viruses, to cells, to whales. On a nanoscale, the biochemical molecules present in life are the scaffolding upon which this higher order builds. The beauty of this system is that it is spontaneous and effortless, and that many of these structures assemble themselves in parallel without directed control. This process of having a set of things assemble themselves into a larger structure, whether it happens, as we described, inside of biology, or if it happens in chemistry, materials science, or physics, is called "self-assembly." Figure 1.5 shows the generic idea. The most important conditions for self-assembly are that you have a library of defined building blocks that are conformationally rigid,[6] and that you have defined non-covalent interactions between these building blocks that will generate something more interesting than a random clump. In biology, proteins are a common building block. As 


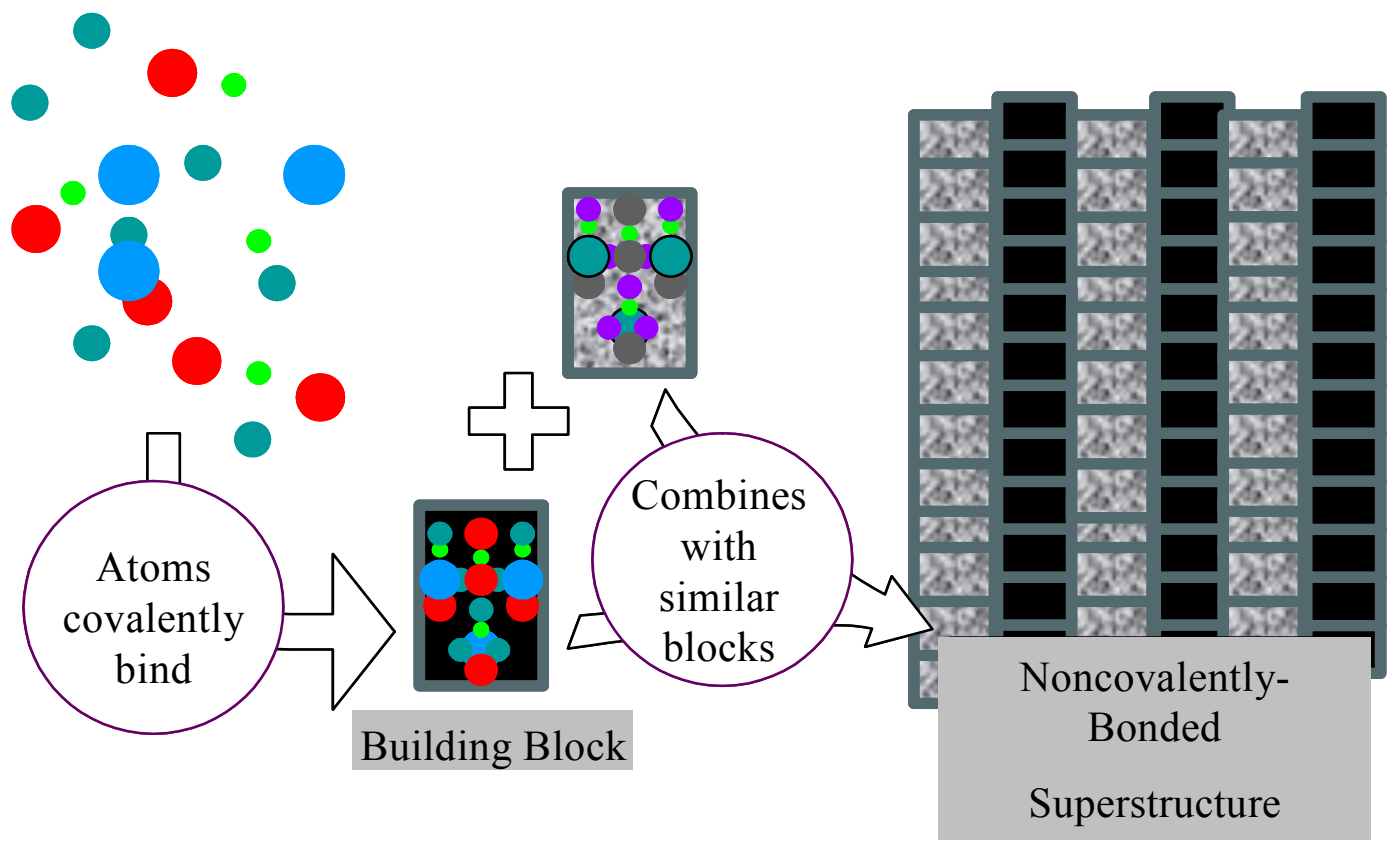

Figure 1.5: Cartoon showing formation of self-assembled structure. Atoms first combine to make defined building blocks. These building blocks are designed so that their properties cause them to self-assemble to form a large, non-covalently bound structure that is the thermodynamic equilibrium assembly for the starting block materials. [6] 
Figure 2.16 illustrates, proteins have a similar size to nanocrystals. Biology also uses RNA, as in the ribosomal unit pictured, lipids, various small molecules, and DNA, our material of interest here.

DNA's ability to self-assemble with itself and with RNA make it indispensable to biology. Self-assembly in DNA is governed energetically by the favorable forces of aromatic stacking between the bases. The double helix has a hydrophobic center that seeks to maximize the stacking forces. The hydrogen bonds do not contribute much to the favorable energetics of the self-assembly process because the hydrogen-bonding groups on the bases are already bonded to water molecules in the solvent before the base-base hydrogen bonds form, making it a fairly even trade for one hydrogen-bond partner over another. The summation of the non-covalent bonds overwhelms the otherwise unfavorable entropics of self-assembly.

\subsubsection{Programmability}

The forces of self-assembly in DNA would be less useful to nanotechnology if they could not be controlled. The sequence of the DNA, while it does not contribute to the energetics of the self-assembly process, does provide specificity to its interactions. We can change the DNA sequence, so we can "program" the sequence to fit our needs.

It would not do any good, however, to change the DNA molecule's sequence if the self-assembly process could not recognize the meaning of the sequence we give it. The DNA's base pairs have many possible hydrogen-bonding interactions they can make. Watson-Crick base pairs, pictured earlier in Figure 1.3, are only two possibilities in a large library of possible pairings between bases. These pairs, however, have the virtue of pro- 
viding a constant distance between the sugar backbone strands in a double helix. It is the helix's desire for a continuation of its structure that maximizes stacking interactions that drives the specific selection of these base pairs in most instances.[50, p. 31]

\subsubsection{Distance control}

The simple, repetitive structure of the DNA double helix provides a method of incrementally controlling the distance between two points of interest (or active sites) on a DNA strand. Insertion or deletion of bases in the strand sequence adds or removes increments of $3.4 \AA$, and the long, $500 \AA$, DNA persistence length makes it quite stiff.[50, p.490] Very few other potential self-assembly building blocks offer such meticulous distance control possibilities.

\subsubsection{Control of surface location}

It is possible to affix DNA to surfaces, either through patterning the surface through standard microlithographic techniques,[51] or by deposition through nanoprobe techniques.[52] In order for nanostructures to be useful, they must have ways to interface with specific locations on a larger size scale. Since we can pattern DNA on a micro and nano scale,[52] DNA can provide a method for interfacing nanotechnology with the surrounding world. 


\section{Chapter 2}

\section{Sample Preparation and}

\section{Characterization}

\subsection{Gold Nanocrystal Synthesis}

Gold nanoparticles may be synthesized as a colloid or sol by reduction of gold salts in solution.[53] Our lab has used a method involving the reduction of $\mathrm{HAuCl}_{4}$ "tetrachloric auric acid." The exact procedure must be calibrated by trial and error to produce a particular desired size. The procedure will yield a product in a range of particle sizes, depending on the amount of tannic acid used, and the coefficient of variation in size is $6.3-16 \% .[54$, pp.18-19] Less tannic acid yields larger particles (up to $17 \mathrm{~nm}) .{ }^{1}$

\footnotetext{
${ }^{1}$ For instance, to produce $5 \mathrm{~nm}$ gold nanoparticles, we put $1 \mathrm{ml}$ of $1 \% \mathrm{HAuCl}_{4}$ in $79 \mathrm{ml} \mathrm{H}_{2} \mathrm{O}$ with a stir bar in an analytically clean roundbottom flask bathed in an oil bath held at $60^{\circ} \mathrm{C}$. In a separate beaker, we heated $4 \mathrm{ml}$ of $1 \% \mathrm{Na}_{3}$ (citrate), $5 \mathrm{ml} 1 \%$ tannic acid, $5 \mathrm{ml} 2.5 \mathrm{mM} \mathrm{K}_{2} \mathrm{CO}_{3}$, and $6 \mathrm{ml} \mathrm{H}_{2} \mathrm{O}$ to $60{ }^{\circ} \mathrm{C}$. When both solutions reached $60^{\circ} \mathrm{C}$, the beaker's solution was added to the roundbottom flask with fast stirring. After about one minute, we raised the temperature to produce a boiling solution, attached a condenser with moderate water flow, and looked for a resulting "reflux line" in the condenser $\left(100-110{ }^{\circ} \mathrm{C}\right)$. At this point, the solution was an attractive red color. The solution was refluxed approximately two hours, and then allowed to cool (still stirred). In this particular procedure, the amount of tannic acid could be varied in
} 
Although this procedure will generate gold nanoparticles with a small size distribution (6-16\% coefficient of variation, depending on size),[54] we avoided the calibration and synthesis steps by purchasing pre-made gold sol solutions of desired sizes from a commercial source. $^{2}[32]$ These particles were supplied in 1 OD solutions in sizes from 2 to $250 \mathrm{~nm}$.

Whether we synthesized the particles or purchased them, the procedure for ligand replacement on the gold nanoparticles was the same. After coating the gold with the sulfonated phosphine ligand, we precipitated the nanoparticles by adding a just sufficient amount of $\mathrm{NaCl}^{3}$ The precipitated solution turned from a red color to purple, and then we centrifuged it in large tubes. ${ }^{4}$ The gold would form a precipitated pellet at the bottom of the centrifuge tube, and then we removed the pale yellow supernatant by decanting or pipetting. With the supernatant, a portion of the smallest impurities in the nanoparticles (that gave the supernatant solution its pale yellow color) were removed, because these particles did not as easily precipitate as the larger red-colored ones.

\section{Properties}

Absorption spectrum Gold nanoparticles larger than about $2 \mathrm{~nm}$ absorb visible light to give a strong reddish color to any solution that contains them. For the sizes of particles used in this study, an absorption maximum was visible at $520 \mathrm{~nm}$, as shown in

a range of $0.01 \mathrm{ml}$ to $5 \mathrm{ml}$. The resulting citrate-coated gold nanoparticles were further stabilized for the presence of salt by coating them with bis(p-sulfonatophenyl) phenylphosphine. The whole reaction was moved to a new flask with a very large excess of 30-50 mg bis(p-sulfonatophenyl) phenylphosphine dihydrate dipotassium salt, and stirred at least overnight, covered.

${ }^{2}$ Ted Pella, Inc. supplied 1 O. D. solutions of gold nanoparticles made by BBInternational (Cardiff, UK).

${ }^{3}$ Over time the sulfonated phosphine ligand slowly hydrolyzes in water to give triphenylphosphine, or is oxidized by air to a phosphine oxide.[53] Neither of these degraded ligands will stabilize the gold colloid in water, so the samples (and the ligand itself) can have a somewhat limited lifetime.

${ }^{4}$ The tubes were centrifuged at $3000 \mathrm{RPM}$ (RCF?) for $\sim 5$ minutes. 


\section{Gold Colloid UV/Visible Absorption Spectra}

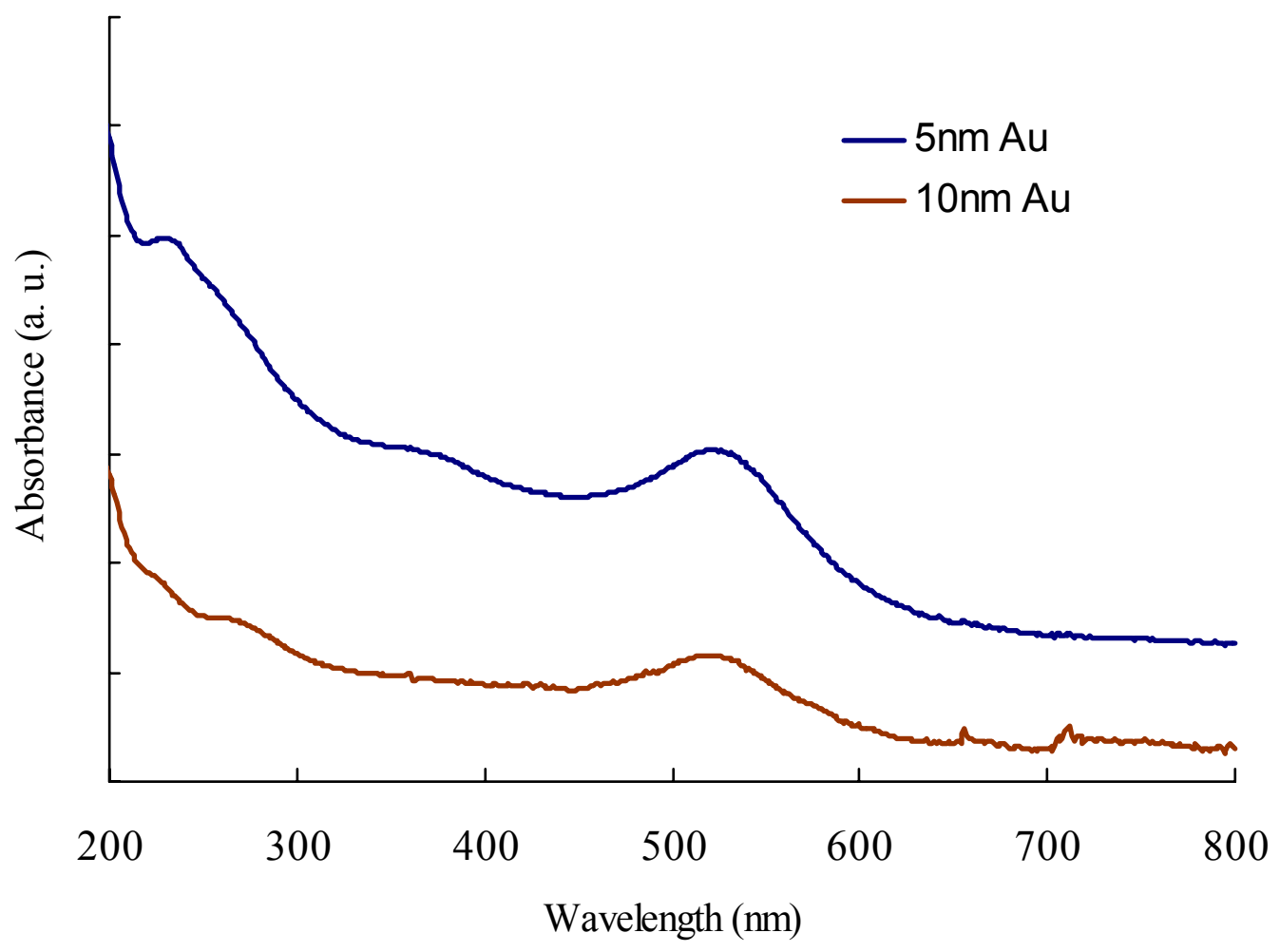

Figure 2.1: Absorption spectra of 5 and $10 \mathrm{~nm}$ gold nanoparticles coated with phosphine ligand, dissolved in water.

Figure 2.1. The extinction coefficients for gold nanoparticles used in these experiments is shown in Table 2.1. We used these extinction coefficients to determine the amount of gold present in any solution.

Size distribution The size distribution of the 5 and $10 \mathrm{~nm}$ gold nanoparticles was about $15 \% .^{5}$

\footnotetext{
${ }^{5}$ Daniela Zanchet, unpublished data.
} 


\begin{tabular}{l|l} 
Nanoparticle Size $\mathbf{( n m})$ & Extinction Coefficient $(\varepsilon)$ at $520 \mathrm{~nm}$ \\
\hline 5 & $9.3 \times 10^{6} \frac{\mathrm{L}}{\mathrm{mol} \cdot \mathrm{cm}}$ \\
10 & $8.7 \times 10^{7} \frac{\mathrm{L} \cdot \mathrm{mol}}{\mathrm{mol} \cdot \mathrm{cm}}$
\end{tabular}

Table 2.1: Extinction Coefficients for 5 and $10 \mathrm{~nm}$ Au nanoparticles at $520 \mathrm{~nm}$

\subsection{Semiconductor Nanocrystal Synthesis}

The semiconductor nanocrystals we used in our experiments were comprised of three separate layers of material. They had cores made of the semiconductor, cadmium selenide. We used a setup such as that shown in Figure 2.2 to synthesize these cores and the outer zinc sulfide shell. To make the cadmium selenide nanocrystal cores, we heated a surfactant, trioctylphosphine oxide (TOPO) to $340-370^{\circ} \mathrm{C}$ under argon or nitrogen in the three-neck flask pictured in Figure 2.2. We then rapidly injected a prepared mixture of selenium and dimethylcadmium in tributylphosphine (TBP) while quickly reducing the temperature to $300^{\circ} \mathrm{C}$ to encourage subsequent growth instead of nucleation. ${ }^{6}$ We could take small aliquot samples and dissolve them into toluene to monitor their absorption as the nanocrystal sizes increased, and stop the reaction at the desired size by reducing the temperature to $90^{\circ} \mathrm{C}$. We precipitated the final core product with anhydrous methanol. Then we transferred it to a septa-sealed vial, and dried it to a paste consistency under nitrogen. ${ }^{7}$

\footnotetext{
${ }^{6}$ The detailed temperatures and quantities in this section come from unpublished notes from Dr. Daniele Gerion, but this procedure parallels the ones described in [33], [4], [34], and [7].

${ }^{7}$ To prepare the synthesis precursors, in a glove box, we mixed $0.148 \mathrm{~g}$ Se and $1.481 \mathrm{~g}$ of TBP, and stirred 10-15 min, completely dissolving all visible grains. We then added $0.37 \mathrm{~g}$ of $\mathrm{Cd}\left(\mathrm{CH}_{3}\right)_{2}$. The $2 \mathrm{~g}$ of this mixture was added to $6 \mathrm{~g}$ TBP, and the vial was sealed with a elastic septum. Outside of the glove box, we stored this precursor mixture in a freezer at $-20^{\circ} \mathrm{C}$ until we needed it. To prepare the surfactant, we placed $2 \mathrm{~g}$ of $90 \%$ (technical grade) TOPO in a 3-necked flask, and purged it three times with nitrogen or argon gas. Still purging, we heated the TOPO to $120^{\circ} \mathrm{C}$, and allowed it to sit, stirring, at that temperature purging for between $20 \mathrm{~min}$. to 2 hours. To conduct the synthesis reaction, we further heated the TOPO to the nucleation temperature of about $360^{\circ} \mathrm{C}$. Then we removed the heating mantle and very rapidly injected 1 (or up to 2) $\mathrm{ml}$ of the precursor stock solution. We stabilized the dropping temperature at $300^{\circ} \mathrm{C}$,
} 


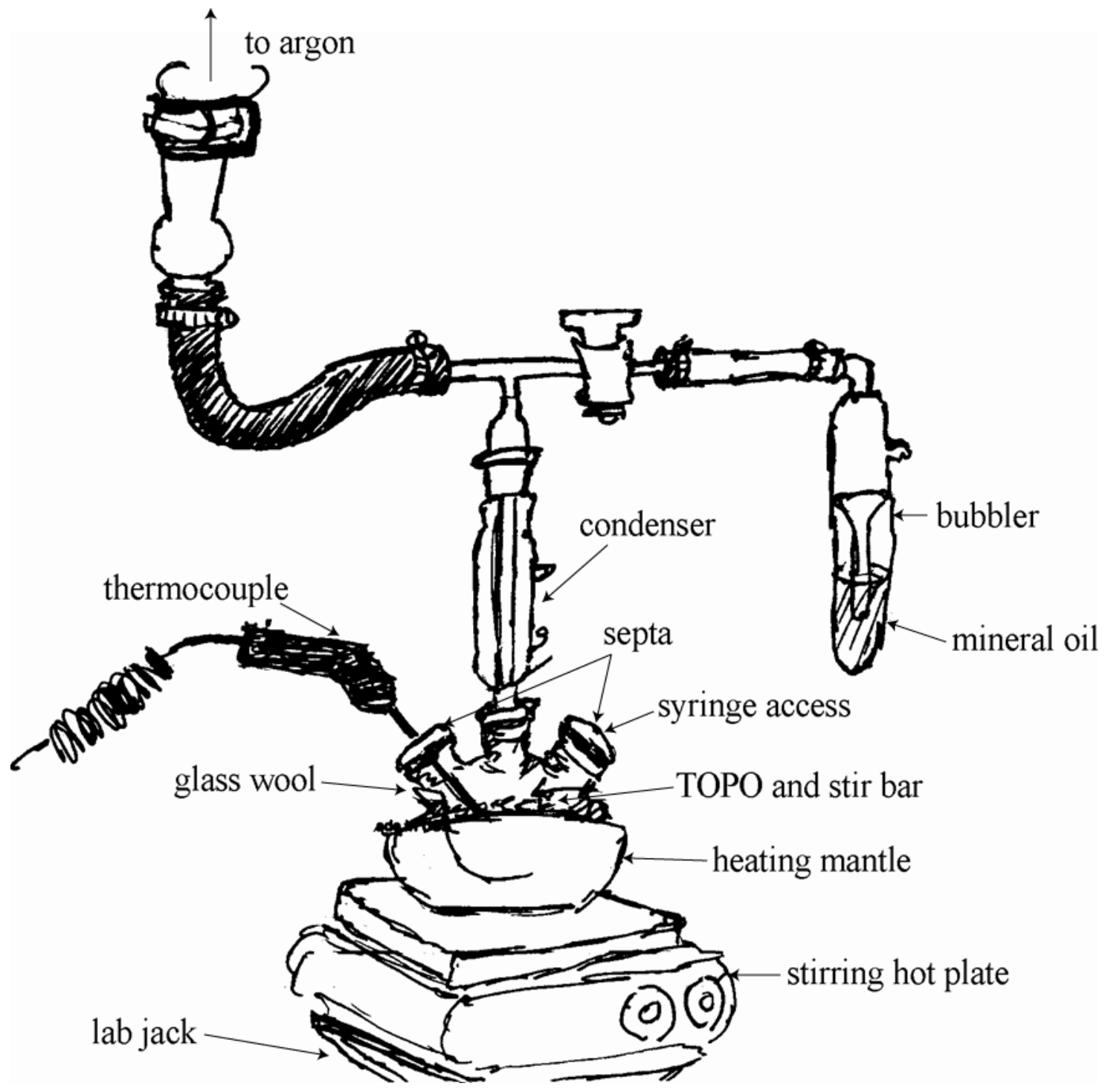

Figure 2.2: This figure shows the typical setup for performing synthesis of CdSe nanocrystals. We added the outer ZnS shell, subsequently, on the same assembly. 
To make the outer zinc sulfide shells, ${ }^{8}$ we prepared, under argon, a stock solution of diethylzinc in TBP. We also purged and heated TOPO in a three-neck flask for a few hours under vacuum with the full Schlenk line setup shown in Figure 2.3. After reducing the TOPO temperature to $60^{\circ} \mathrm{C}$, we injected a prepared solution of 20-40 mg CdSe nanocrystals in pentane. Then we pumped to remove the pentane, and purged with nitrogen before heated the mixture to $160^{\circ} \mathrm{C}$. Separately, we added hexamethyldisilathiane $\left[(\mathrm{TMS})_{2} \mathrm{~S}\right]$ (as a source of sulfur) to the diethylzinc solution. Then we dropwise injected this mixture into nanocrystal/TOPO mixture in the three-neck flask to grow the shells around the nanocrystals. We could monitor the growth of the shells by checking the absorption and fluorescence spectra versus time. After the shell growth was finished, we could leave the reaction at $90^{\circ} \mathrm{C}$ for a while before adding a few milliliters of butanol to prevent the TOPO from solidifying, and then cooling the reaction to room temperature. These nanocrystals

and transferred small samples quickly to toluene filled cuvette to monitor how the absorbance changed over time. As we approached the absorbance value that corresponded to the size we wanted, we reduced the flask temperature to $90^{\circ} \mathrm{C}$, and waited for the nanocrystal reaction to cool. Then we reduced the temperature further to $35^{\circ} \mathrm{C}$. We precipitated the nanocrystals without stirring with anhydrous methanol, and then removed any excess with a syringe. The remaining solution we stirred and transferred to a septum-sealed vial, and then purged the vial with nitrogen. Then we centrifuged the vial to pellet the nanocrystals and used a syringe to completely remove most of the excess methanol supernatant. This pellet was dried to a paste in the purged vial under nitrogen.

${ }^{8}$ To make the shells, we mixed in a glove box $3.3 \mathrm{~g}$ TBP and $1.26 \mathrm{~g} \mathrm{ET}_{2} \mathrm{Zn}$ in a scintillation vial, and sealed it with a septum. We also prepared a septum-sealed vial with only TBP. Then we put $4 \mathrm{~g}$ of TOPO into a 3 -neck flask and purged, replacing air with argon (or nitrogen). Then we switched to vacuum and heated the TOPO to about $60{ }^{\circ} \mathrm{C}$ for $30 \mathrm{~min}$. We heated the TOPO to $120^{\circ} \mathrm{C}$ for at least $2-3$ hours (still under vacuum). We reduced the temperature to $60^{\circ} \mathrm{C}$ and replaced the vacuum with argon. We placed $20-40 \mathrm{mg}$ of the previously prepared CdSe cores in a purged, septum-sealed vial, and dissolved them in $0.5 \mathrm{ml}$ of pentane. To move the nanocrystals into the TOPO in the 3-neck flask, we first injected $0.5 \mathrm{ml}$ of the previously prepared pure TBP, and then the $0.5 \mathrm{ml}$ pentane/nanocrystal mixture. We pumped out the pentane, and then purged with argon after there were no more bubbles. We increased the flask temperature to $160^{\circ} \mathrm{C}$. Then, inside the hood, we added a (strongly smelly) $0.36 \mathrm{ml}$ (TMS) ${ }_{2} \mathrm{~S}$ to the prepared diethylzinc/TBP solution. We diluted $0.5 \mathrm{ml}$ of this solution into $0.5 \mathrm{ml}$ of TBP inside of a syringe, shaking the syringe to ensure good mixing. We added this syringe mixture very slowly (dropwise) to the prepared nanocrystals in TOPO. It took about five minutes to empty the syringe. We repeated this syringe mixing/injection step three more times (= 20 minutes, total, spent injecting). After we had the desired fluorescence properties, we reduced the flask temperature to $90^{\circ} \mathrm{C}$, added $3-4 \mathrm{ml}$ butanol, and then cooled the reaction mixture to room temperature. To precipitate the core/shell nanocrystals, we took $2 \mathrm{~g}$ of the reaction mixture and added about $20 \mathrm{ml}$ of anhydrous methanol. 


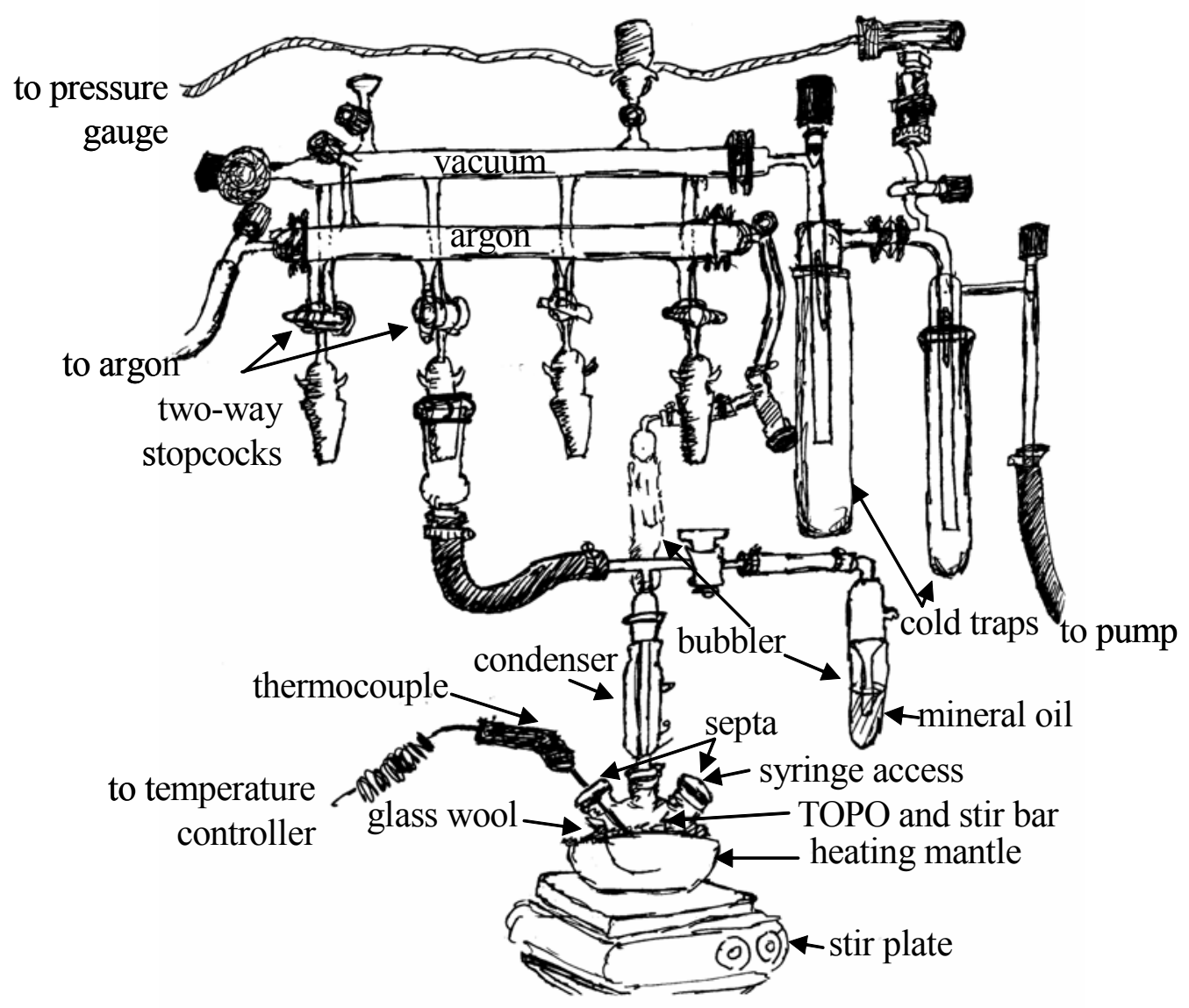

Figure 2.3: A sketch of the CdSe core and ZnS shell complete synthesis setup, attached to a Schlenk line that provided access to both vacuum and argon. 
could be once again precipitated with anhydrous methanol and dried to a paste.

To make these hydrophobic core/shell nanocrystals both water-soluble and biocompatible, we coated them with a siloxane layer..$^{9}[34][7]$ Figure 2.4 shows an outline diagram of this coating procedure. We put the core/shell nanocrystals in a solution of dimethyl sulfoxide (DMSO), anhydrous methanol, mercaptopropyltrimethoxysilane (MPS), and tetramethylammonium hydroxyl pentahydrate $\left[\left(\mathrm{CH}_{3}\right)_{4} \mathrm{NOH} \bullet 5 \mathrm{H}_{2} \mathrm{O}\right](\mathrm{TMAH})$ and vortexed or sonicated it until it was clear. Then we put the mixture under nitrogen in a large three-neck flask, stirring to prime the nanocrystal surfaces with an initial MPS layer. After at least an hour, or overnight, we gently heated it to $60^{\circ} \mathrm{C}-70^{\circ} \mathrm{C}$ under a cooled condenser for a half-hour to crosslink the silanols into siloxane bonds. Then we added a mixture of methanol, water, aminopropylsilane (APS), and (trihydroxysilyl)propyl methylphosphonate to the reaction at room temperature, leaving it for two hours, to introduce more components to the outside layer. Then we again heated the solution to $60-70^{\circ} \mathrm{C}$ to crosslink the silanol

\footnotetext{
${ }^{9} \mathrm{~A}$ typical synthesis procedure, as described in unpublished notes from Dr. Daniele Gerion, or in [7] would proceed in this manner. We would precipitate $1 \mathrm{ml}$ of butanol/TOPO core/shell reaction mixture with anhydrous methanol, and then dissolve the precipitate in $50 \mu \mathrm{l}$ of MPS and vortex the solution. Then we would add $5 \mu \mathrm{l}$ of TMAH in methanol to get a clear solution. Then we diluted the mixture with 120 $\mathrm{ml}$ of anhydrous methanol brought to $\mathrm{pH} \sim 10$ with $750 \mu \mathrm{l}$ of TMAH, and put the mixture, stirring in a 500 ml 3-neck flask under nitrogen to prime nanocrystal surface with MPS. We then heated the flask under a cooled condenser to $70^{\circ} \mathrm{C}$ for $30 \mathrm{~min}$, and then allowed the reaction to cool. Next, we added $180 \mathrm{ml}$ of methanol, $20 \mathrm{ml}$ water, $40 \mu \mathrm{l}$ of APS (alternatively, $40 \mu \mathrm{l}$ of MPS or a mix of $20 \mu \mathrm{l}$ each of MPS and APS), and $1 \mathrm{ml}$ of $42 \%$ (wt/wt) trihydroxysilylpropyl methyl phosphonate (monosodium salt) in water, and allowed the layer to build for two hours. ( We could replace the phosphonate compound with poly(ethylene glycol) silane or trimethoxysilylpropyltrimethylammonium chloride to change the charge properties of the surface.) Then we again heated under a cooled condenser to $60^{\circ} \mathrm{C}$ to form more siloxane bonds. After the reaction cooled again, we added a solution of $4 \mathrm{ml}$ chlorotrimethylsilane in $36 \mathrm{ml}$ methanol, basified with TMAH to a $\mathrm{pH}$ of 9-10 to quench any remaining free silanols. After heating to $60^{\circ} \mathrm{C}$ again for 30 min., we let the reaction cool and sit, stirring, overnight to a few days. We removed remaining excess silane with centrifugal filters (Centriplus-20, MWCO 100,000), and the solution was condensed to a final volume of 2 $\mathrm{ml}$. The solution was then dialyzed against methanol in 10,000 MWCO dialysis tubing for a day, and filtered to remove aggregates through a $0.45 \mu \mathrm{m}$ syringe filter. After at least 12 hours, we could move the finished nanocrystals to water in a NAP column or a $20 \mathrm{~cm}, 0.7 \mathrm{~cm}$ diameter column filled with $5 \mathrm{~g}$ of Sephadex G25 gel medium, and pre-equilibrated with $10 \mathrm{mM}$ phosphate buffer $(\mathrm{pH} \sim 7)$. We collected the fluorescent portion of the eluent, and filtered the collected fraction through a $0.22 \mu \mathrm{m}$ acetate filter to get our fin
} 


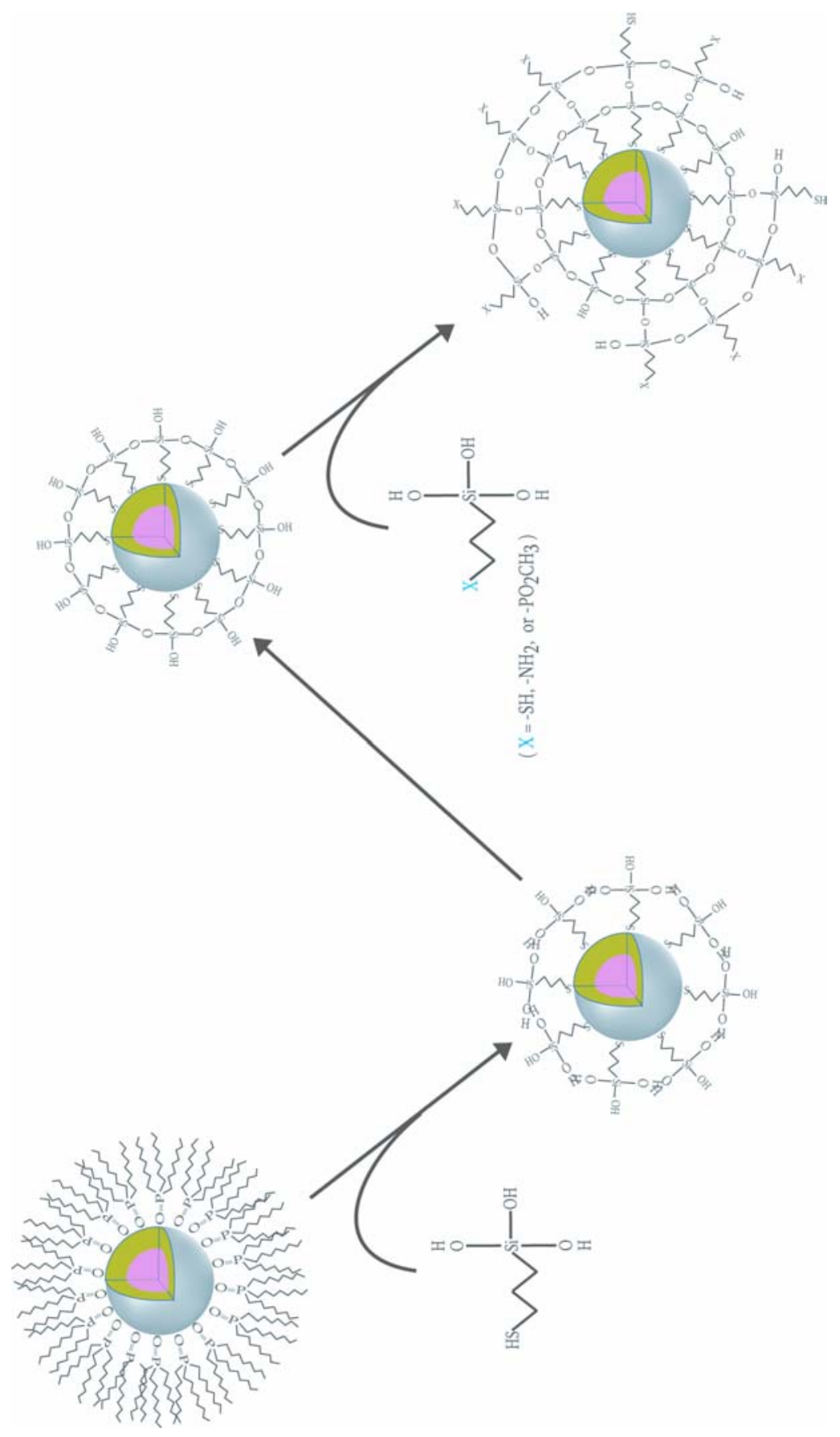

Figure 2.4: Diagram showing the steps for creating a siloxane layer on the outside of core/shell nanocrystals. In the first step, mercaptopropylsilane primes the surface of TOPO-coated nanocrystals. Then base hydrolyzes the silanol groups to form siloxane bridges. The reaction can incorporate different functional groups ("X") in the outside coating of the layer in the final step by addition of an appropriate reagent. 
bridges. To quench the remaining reactive groups, we added basified chlorotrimethylsilane in methanol, allowing it to react for two more hours. We again heated the reaction mixture for 30 minutes at $60-70^{\circ} \mathrm{C}$, and then cooled it to room temperature. We allowed the cooled reaction mixture to sit at least overnight up to four days, stirring under a nitrogen atmosphere. We dialyzed the solution against methanol for a day to remove excess reactants, and then filtered it to remove any large aggregates. More excess free silane was removed by flushing in centrifugal filters with methanol to condense the solution to a final volume of about two milliliters. After another half-day, we could move the siloxane-coated core/shell nanocrystals into water in a solvent exchange column.

Because of the long and involved multi-day procedure to prepare these samples, most of our DNA templating experiments used gold nanoparticles instead of these fluorescent semiconductor samples. These siloxane-coated nanocrystals were more salt-stable, however, than the phosphine-ligand-coated gold nanoparticles, and could withstand 200 $\mathrm{mM} \mathrm{NaCl}$, or even as much as $1 \mathrm{M} \mathrm{NaCl}$, depending on the sample.[7] It is possible we would have had better success with the lattice experiments described in Chapter 4 if we had used these more-salt-stable particles, but the procedure to make these particles was not sufficiently robust to contemplate using them until late in the course of the lattice project.

The optical properties of these siloxane-coated particles were similar to the hydrophobic starting material. Figure 2.5 shows the absorption and emission spectra for a series of silanized CdSe/ZnS core/shell nanocrystals in a buffer solution.

Depending on which reagents were used in the process of making the siloxane coating, it was possible to alter the charge characteristics of the surface, as discussed later 


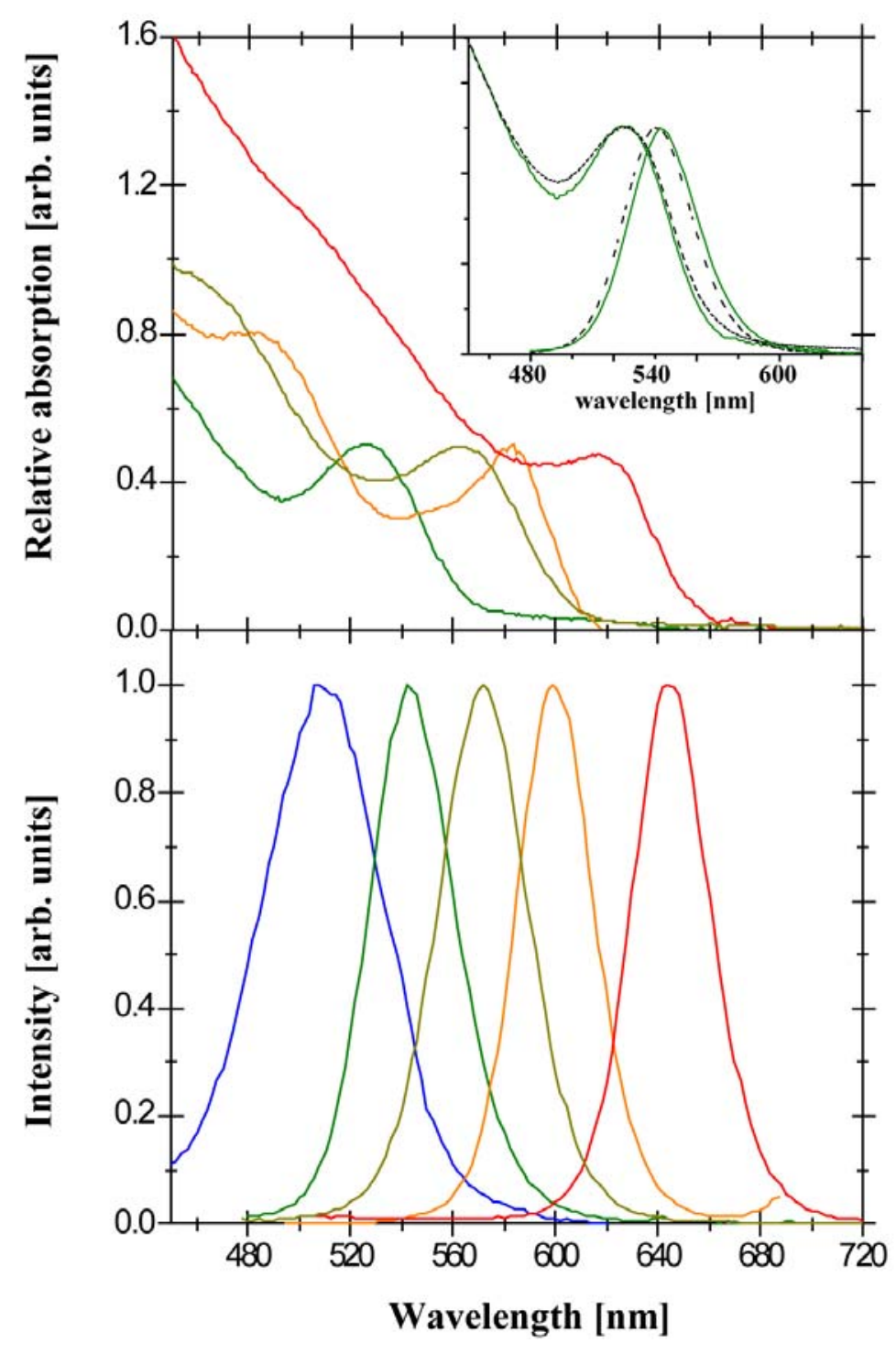

Figure 2.5: Figure from Gerion, et. al. [7] showing the absorption and emission spectra of a set of red, orange, yellow, green, and blue silanized CdSe/ZnS nanocrystals. The upper half of the figure contains absorption spectra, and the lower half contains emission spectra. The top-right inset shows an example of the very small difference for the absorption and emission profiles in a sample of green fluorescencing nanocrystals, before (in dashed lines) and after (in solid lines) siloxane coating. 
in Section 2.3.2. The functional groups on the outside of the nanocrystals could also be used to bind DNA strands[35][51][55] or proteins[34], or to promote non-specific binding inside of cells[34] in cell labeling experiments.[56]

\subsection{DNA Synthesis}

The chemical synthesis of DNA is entirely automated. We purchased most strands, including all thiol-modified strands, from Integrated DNA Technologies (IDT). Some strands, including all of the branched strands, and some of the simple linear complements we synthesized ourselves on an Applied Biosystems Expedite 8909 synthesizer.

Regardless of whether a strand was received from a commercial supplier, or was one we ourselves synthesized, the process of synthesizing a DNA strand involved stepwise addition of protected monomers called "phosphoramidites." The phosphoramidites are comprised of the DNA sugar and base (a "nucleotide"), with its reactive amino and hydroxyl groups protected. The reactive primary amines on cytosine and adenine are protected with benzoyl groups, and the primary amine on guanine is protected with a isobutyryl group. These protection groups are removed in the last stage of synthesis through treatment with ammonium hydroxide, which also cleaves the completed strands from the support. The first base is attached to a solid support of controlled pore glass by the 3 ' end of the deoxyribose sugar. The 5' end of the sugar has its hydroxyl group protected by a dimethoxytrityl (DMT) group.[49]

The DMT group is removed in the first "deblocking" stage of synthesis that treats the growing DNA strand, on solid support, with trichloroacetic acid in dichloromethane.[57] 
The deblocking step makes the 5' hydroxyl group on the deoxyribose sugar available for the next stage of the reaction.

The second step is the coupling of the next deoxymononucleotide phosphoramidite in a solution of tetrazole and acetonitrile to make a phosphite triester bond. Unreacted 5' hydroxyl groups (from failed coupling) are capped with an acyl group in the third step with a mixture of acetic anhydride and N-methylimidazole in pyridine and tetrahydrofuran $(\mathrm{THF})$.

The final step in the synthesis cycle is the oxidation of the unstable phosphite triester linkage between the sugars with a solution of iodine in THF, water, and pyridine. The oxidation reaction gives the desired product of a phosphate triester.

This four-stage-cycle, accompanied by washes in between steps, is repeated to add additional bases to the synthesized DNA strand. The synthesis cycle adds all of the reagents in excess in order to drive the individual reactions steps to completion.[58] The final product, without an additional phosphorylation step, has a hydroxyl group on both the $5^{\prime}$ and the $3^{\prime}$ ends of the strand. If we desire to use the strand for a ligation reaction (where two DNA strands are joined together, end-on-end, using an enzyme, (as Dr. Gerion and I, and then C. Micheel, discovered the hard way), we must phosphorylate the 5' end of the strand, either during the solid phase synthesis, or afterwards using T4 polynucleotide kinase.[42, p. 132] The kinase reaction also would allow the DNA to be radiolabeled, although no experiments described here use this procedure.

The entire synthesis process, except for the final treatment with ammonium hydroxide is, thankfully, automated. A control terminal allows the user to control the sequence 
of the entire DNA strand, and then monitor the likely success of their synthesis reaction with a trityl monitor. The trityl group that protects the 5' hydroxyl group on each DNA sugar is a convenient chromophore that absorbs at $498 \mathrm{~nm}$. As the reaction proceeds, an in-line trityl monitor measures the amount that the removed trityl groups absorb as they come off the growing strand in the deblocking step. By comparing the trityl absorbance for successive deblocking steps as the strand grows, it is possible to estimate the success of the strand growth. Bad luck can stem from poor purging of the system prior to synthesis, thus allowing water to interfere with the reaction. Water-contaminated reagents can also cause problems. The phosphoramidites themselves have a short shelf-life, and a degraded phosphoramidite can spoil an entire synthesis batch. The trityl yield will decrease each time a base of the degraded type is added.[59]

The trityl assay does not detect all possible problems, however, and the final product in even a well-run reaction will be contaminated to some extent with failure sequences.

\subsubsection{DNA purification}

Synthesized DNA must be purified to remove salt, the cleaved protecting groups, failed incomplete products, and any modified oligonucleotides that resulted from undesired side-reactions. The type of purification necessary depends on the purpose for the DNA.

Because of the high efficiency of the synthesis process, the reaction yield is quite high, especially for short strands. For many purposes, although not the ones described here, only desalting through ethanol or butanol precipitation is necessary. This procedure removes salt and most of the cleaved blocking groups.

For DNA that we used in these experiments, greater purification was necessary. 
The type of purification we used depended on whether we made the DNA ourselves, or ordered it commercially. It also depended on which experiment it was designed for, and how long it was. An easy and effective method for purification of DNA from failure sequences was to leave the last trityl group on the strand, and then to use its hydrophobicity to separate it with commercially available "Poly-Pak" cartridges from Glen Research.[60] This procedure generally yielded a product $90-95 \%$ pure.

For more rigorous purification we used, or asked our commercial supplier to use, reverse phase high pressure liquid chromotography (RP-HPLC). This procedure once again relied on the presence of the terminal trityl protecting group. It can resolve N-1 contaminants for strands up to 40 bases in length.

Longer strands, or the strands having a branched structure, required the most strenuous purification, because they were more highly contaminated with undesired products, and because these undesired products were more difficult to differentiate from the desired strands. We used denaturing polyacrylamide gel electrophoresis (PAGE) purification to purify these types of strands. By using a gel of sufficient length, and by varying the gel percentage, it was possible to remove the N-1 contaminants in even the longest strands used for these experiments (100 bases). For the experiments described in Chapter 4.2, the DNA was self-assembling into a large-length-scale structure, and any impurities in the DNA would interrupt, and probably terminate, the self-assembly process. $95 \%$ purity was inadequate for these experiments, and commercial PAGE purification was inadequate, as well. All of these DNA strands were purified in-house in denaturing PAGE gels. 


\subsubsection{Gel electrophoresis}

Gel electrophoresis was indispensable to the experiments described here. Figure 2.6 shows an overview of the gel electrophoresis setup and procedure for DNA gels. The separation procedure takes place on a support medium, which is a porous material. The figure shows polyacrylamide which was, for us, a 19:1 mixture of acrylamide to N,N'-methylenebis-acrylamide. The bisacrylamide creates crosslinking in the polymer matrix. We also used agarose as a support medium for some (mostly non-DNA) samples. The support medium is immersed in a buffered water solution, the sample is loaded into starting wells (see \#1 in Figure 2.6), and then the whole gel/buffer/sample system is hooked up to a DC power supply (\#2). The samples move through the gel matrix at a rate that was increased by their charge, but decreased by their mass. Two molecules with the same charge and mass may even move at different rates through the gel if they have a different shape.

The current and voltage supplied during the electrophoresis process depended on decisions balancing the desire to avoid excessive joule heating from the passage of current through the gel, and the desire to avoid allowing the bands the time to diffuse during separation.

The buffer system which makes contact with the gel provides a conduction medium for the current, and buffers reactions that occur at the positive and negative electrodes. When electrons leave the negative electrode for the solution medium, they electrolyze the water and generate hydrogen gas and base:

$$
2 e^{-}+2 \mathrm{H}_{2} \mathrm{O} \rightarrow \mathrm{H}_{2}+2 \mathrm{OH}^{-}
$$



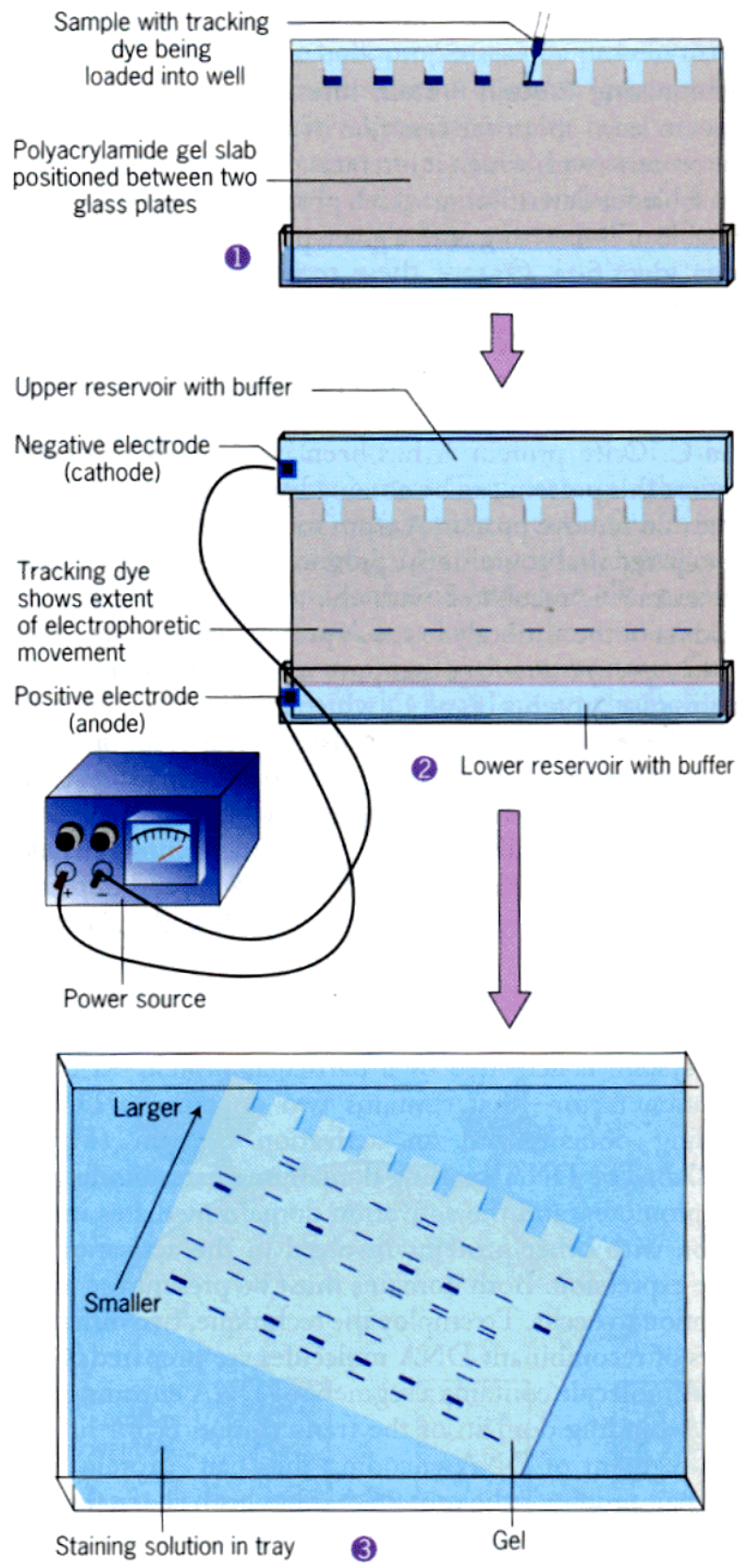

Figure 2.6: Steps for running a DNA polyacrylamide vertical gel. (from Karp: Cell and Molecular Biology)[8, p.762] 
Electrons leave the solution at the positive electrode, making oxygen gas, and acid:

$$
\mathrm{H}_{2} \mathrm{O}-2 e^{-} \rightarrow \frac{1}{2} \mathrm{O}_{2}+2 \mathrm{H}^{+}
$$

If the gel solution were not buffered, this set of reactions would create a $\mathrm{pH}$ gradient, and the separation of the sample would not proceed evenly, as intended. During long gel runs, the buffer was recirculated to refresh it.

The choice of buffer system centered on the $\mathrm{pH}$ desired to give the sample a good charge. We performed most of the electrophoresis in these experiments at a $\mathrm{pH}$ of 8 , in a tris-borate-EDTA (TBE) buffer ${ }^{10}$, which has a high buffering capacity and a low conductivity.[61, p. 69] The EDTA (ethylenediaminetetraacetic acid) has four carboxyl groups and two amino groups that chelate metal ions, $[62$, p. 288] thereby inhibiting nucleases that would break down the DNA.

\section{Denaturing polyacrylamide gel electrophoresis}

Denaturing electrophoresis, mentioned earlier in Section 2.3.1, involved including high concentrations of urea in the gel matrix, and the presence of formamide in the loaded sample solution in order to interrupt any base pairing in the samples during the gel run. ${ }^{11}$ The gels were also run at $50{ }^{\circ} \mathrm{C}$ using a recirculating water bath system to maintain the temperature of the running buffer. This process is shown in Figure 2.7. ${ }^{12}$ The high

\footnotetext{
$10 " 1 \mathrm{X}$ " TBE buffer is $89 \mathrm{mM}$ Tris base, $89 \mathrm{mM}$ boric acid, and $2.5 \mathrm{mM}$ EDTA (pH 8.3). We diluted 10X and 5X TBE stock solutions. 10X stock tends to precipitate over time.

${ }^{11}$ Denaturing gels were prepared from $20 \%$ and $0 \%$ stock solutions. $250 \mathrm{~g}$ urea $(50 \%$, w/v) was mildly heated with stirring in $250 \mathrm{ml} \mathrm{40 \%} \mathrm{19:1} \mathrm{acrylamide/bisacrylamide} \mathrm{solution} \mathrm{(Bio-Rad)} \mathrm{and} 50 \mathrm{ml} 10 \mathrm{X}$ TBE to make $500 \mathrm{ml}$ of $20 \%$ stock. $0 \%$ had $250 \mathrm{ml}$ deionized water instead of the $40 \%$ acrylamide/bisacrylamide solution. By mixing ratios of these starting stock solutions, denaturing gels of 3-20\% could be prepared. Practically, gels of less than 6\% were fragile and difficult to handle, and thus rarely, if ever, used. For loading, DNA samples were dried and then resuspended in solution of $90 \%$ formamide, $10 \mathrm{mM} \mathrm{NaOH}$, with a small amount of xylene cyanol FF blue dye to help with loading and tracking. Resuspended samples were vortexed, and then heated to $90^{\circ} \mathrm{C}$ for 3 min to ensure complete denaturation.
} 


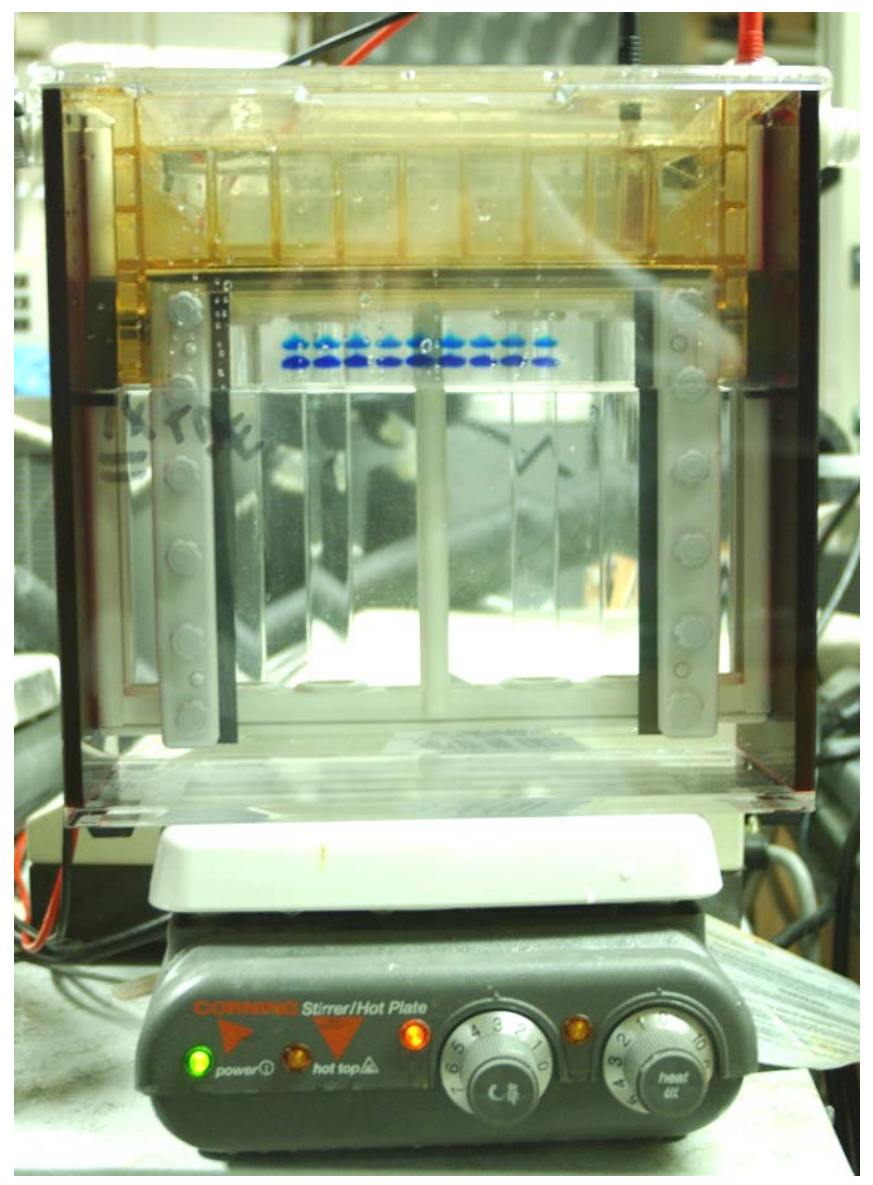

Figure 2.7: Polyacrylamide gel running in a temperature-controlled buffer bath. The blue bands seen in the gel are two kinds of dyes: xylene cyanol FF (top band), and bromophenol blue (bottom band) that are merely markers that allow the user to track the progress of the gel run. 


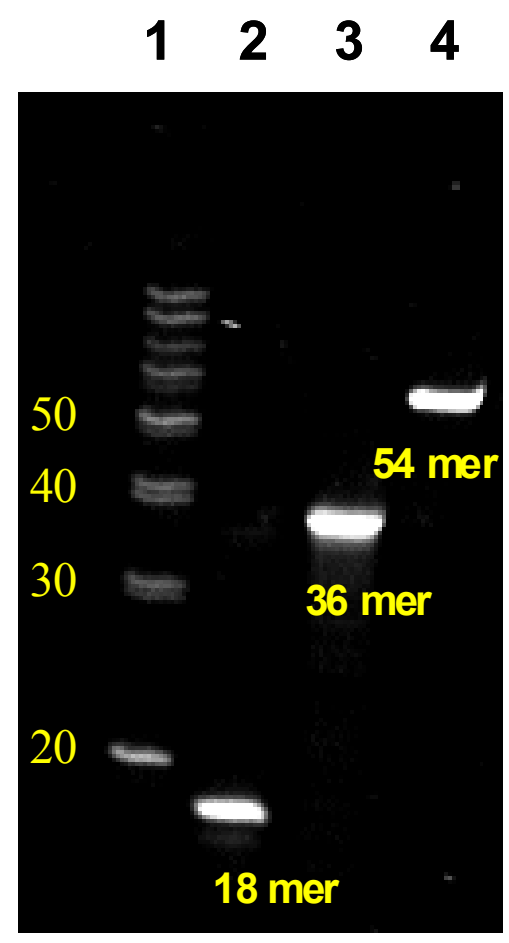

Figure 2.8: Example of a DNA-containing, 20\% denaturing PAGE gel showing four lanes loaded (lane 1, 20 bp ladder (denatured); lane 2, an 18 base single strand; lane 3, a 36 base strand; lane 4 , a 54 base strand).

running temperature and denaturing conditions are designed to ensure the DNA runs as single strands. This sort of gel electrophoresis is useful to verify the length of a DNA strand after synthesis or receipt from the manufacturer, as Figure 2.8 shows in a sample gel. ${ }^{13}$

As described earlier, denaturing gel electrophoresis can purify DNA. For purification, a gel with a total percentage suitable for the length of the DNA strand (longer strands need lower percentage gels) is poured in a thickness from $0.75 \mathrm{~mm}$ to $3 \mathrm{~mm}$. The thicker

\footnotetext{
${ }^{12} 16 \mathrm{X} 16 \mathrm{~cm}$ vertical gels were run in a Hoefer SE 600 Standard Dual-Cooled Gel Electrophoresis Unit purchased from Amersham Pharmacia Biotech. The unit allowed for cooling or heating of the lower buffer reservoir through an internal glass-tubed heat exchanger and for recirculation of the buffer from the lower to upper chambers for longer runs.

${ }^{13}$ DNA sequences $\left(5^{\prime} \rightarrow 3^{\prime}\right)$ for lanes $2-4$ were GTC ACA TAG CGT TAC TGC, CAA CAT CCT CGA ACG AGC GTC ACA TAG CGT TAC TGC, CAA CAT CCT CGA ACG AGC CAA CAT CCT CGA ACG AGC GTC ACA TAG CGT TAC TGC.
} 
the gel, the greater the amount we could load, but also the lower the ability of the gel to retain a homogenous environment for the DNA migration. Thicker gels tended to cool somewhat unevenly, so that bands were not as well-resolved. Most of our purification gels were $0.75 \mathrm{~mm}$ thick for this reason; a gel this thin allowed removal of detectable amounts of N-1 strand-length contamination. The 16 X $16 \mathrm{~cm}$ gels were run for several hours at 500V and $50{ }^{\circ} \mathrm{C}$ until the dye bands had migrated sufficiently so that the expected migration of the desired DNA would be a short distance from the gel bottom. The gels were stained in a buffer bath (see Figure 2.6 \#3) containing a proprietary DNA stain called "GelStar."14 After staining, the purification prep gels were transilluminated with UV light and photographed. The desired band tended to be the most prominent band on the gel, unless the reaction had a significant failure point (such as, perhaps, in a branched structure). We isolated the desired product from the gel by excising the portion of the gel containing the product, carefully avoiding inclusion of failure products also visible in the gel, as shown in Figure 2.9. The DNA was eluted from the excised gel pieces, butanol-extracted, and then ethanol-precipitated to isolate the DNA from the solution, dyes, and salt. ${ }^{15}$ Each purification gel was limited to a loading of 100-140 $\mu \mathrm{g}$ of DNA. This small gel loading

\footnotetext{
${ }^{14}$ GelStar ${ }^{\circledR}$ Nucleic Acid Gel Stain was purchased from FMC Bioproducts or BioWhittaker Molecular Applications or Cambrex, and prepared according to the manufacturer's instructions. It is a proprietary fluorescent intercalating DNA stain that stains double-stranded DNA more effectively than single-stranded. It is $4-16 \mathrm{X}$ more sensitive than the more common stain, ethidium bromide, for double stranded DNA, and 20-80X more sensitive for single-stranded DNA when excited a $300 \mathrm{~nm}$ UV light source. Its excitation maximum is $493 \mathrm{~nm}$, and emission maximum is at 527 (for DNA).[63]

${ }^{15}$ Extracted pieces from $\frac{1}{2}$ of a gel were placed in a $1.5 \mathrm{ml}$ Eppendorf tube in $500 \mu \mathrm{l}$ elution buffer (500

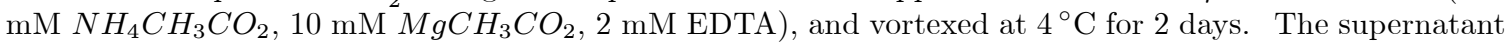
was then transferred to a new $1.5 \mathrm{ml}$ Eppendorf tube, along with a rinse of $200 \mu \mathrm{l}$ deionized water. For the butanol extraction, twice, $700 \mu \mathrm{l}$ n-butanol was added, vortexed, centrifuged, and removed; a third time 800 $\mu \mathrm{l}$ n-butanol was added, vortexed, centrifuged, and removed, and then a fourth and final time, $600 \mu \mathrm{l}$, which was carefully removed. About $250 \mu \mathrm{l}$ of solution remained, to which $1200 \mu \mathrm{l}$ ethanol was added, and the vial was frozen on dry ice for 30 min., and centrifuged at 14,000 RPM to precipitate the DNA. The ethanol was then decanted, and then $1 \mathrm{ml}$ of $70 \%$ cold ethanol was added, and the vials centrifuged 6 additional min before decanting the supernatant, again. The DNA was then vacuum centrifuged to give a dry, desalted, purified DNA product.
} 

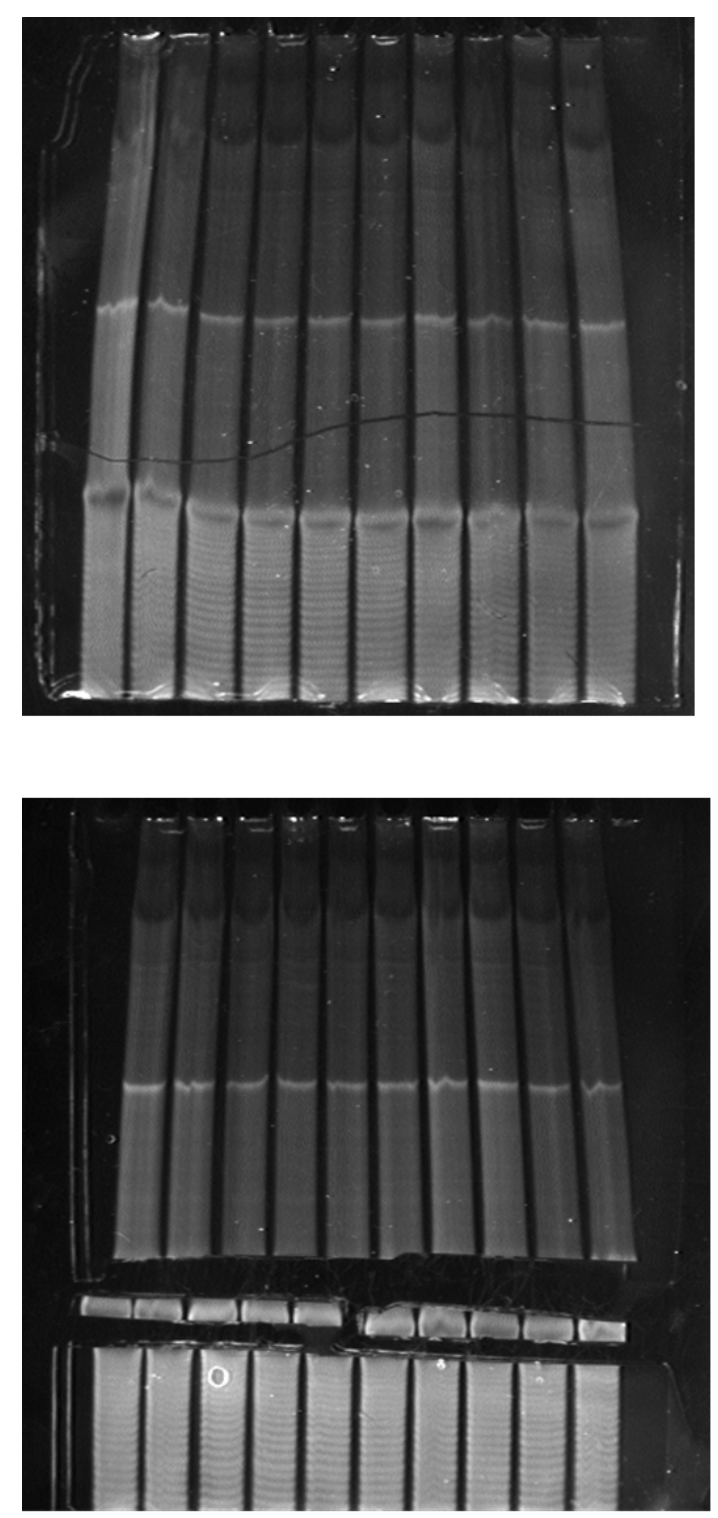

Figure 2.9: Purification of 54 base DNA in 15\% denaturing PAGE. The top image shows the gel before cutting, and the bottom shows the excised band. The bands below the excised band are the shorter failure contaminants of the synthesis reaction that we are trying to remove. (The line across the top gel was a fracture caused by the process of transfering the gel onto the transilluminator for imaging. It did not affect the results.) 
capacity makes preparative PAGE a labor-intensive process. Many, many gels had to be prepared to purify even the product from a single 1 umole-scale synthesis. Only as many gels were prepared as were necessary to yield sufficient product for a desired experiment. Even then, with each set of gels taking 2.5-4 hrs. to run, and with three gel units running simultaneously, preparative PAGE tended to be a "character-building," tedious, repetitive task.

Denaturing gel electrophoresis also provided a method to check the purity of purified DNA. For strands that had been purified, given the large scale of the task, it was necessary to verify that the purification procedures had been successful so that detectable amounts of contaminant did not remain, and to confirm that the vials had not been accidentally mixed or interchanged during the multi-day process. Figure 2.10 shows a sample of a purity check for a variety of DNA strands. ${ }^{16}$ Alternate lanes compare the starting, impure, synthesis product and the PAGE-purified product. The goal for this check was to have no visible lower bands in the lanes containing purified DNA.

\section{Native polyacrylamide gel electrophoresis}

In some cases, it was desirable to run gels in "native" conditions. These conditions keep complementary DNA sequences base-paired. These gels were run in the absence of urea, formamide, or other denaturing agents, and the gel was cooled at $4{ }^{\circ} \mathrm{C}$ to prevent joule-heating from separating the DNA strands. Figure 2.11 shows an example of a native

\footnotetext{
${ }^{16}$ These gels were stained with "Stains-all" to allow white-light imaging. (We procured "Stains-all" from Eastman Kodak Company, Rochester, NY.) The DNA is not recoverable after this sort of staining, but the stain is visible by naked eye without UV transillumination. To prepare "Stains-all" staining solution, "Stains-all" was dissolved in $450 \mathrm{ml}$ formamide until there was a dark-purple-colored solution, and then added to $550 \mathrm{ml}$ of buffer to make $1 \mathrm{~L}$ of staining solution which lasts for 6 months stored in the dark. A denaturing gel took $30 \mathrm{~min}$. in this solution to stain, a native gel (described later) took $1 \mathrm{hr}$.
} 


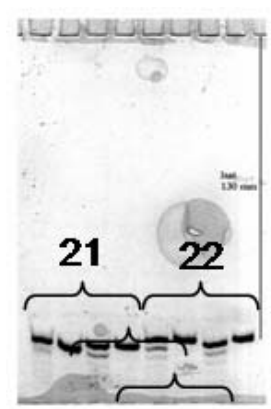

A

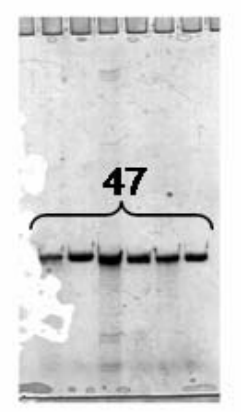

$E$

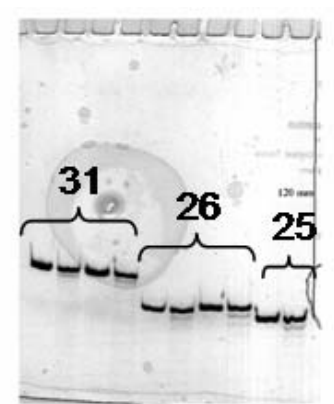

B

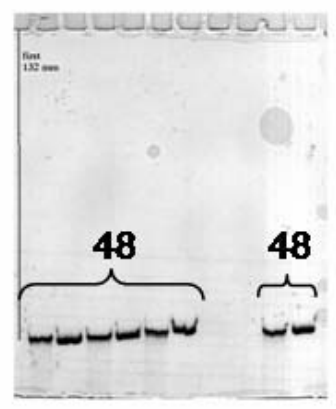

$\mathbf{F}$

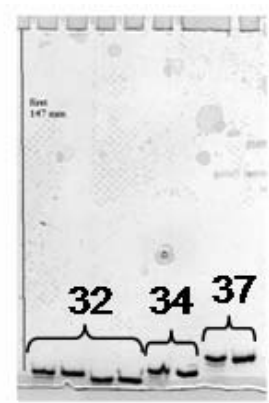

C

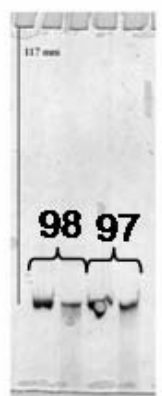

$\mathbf{G}$

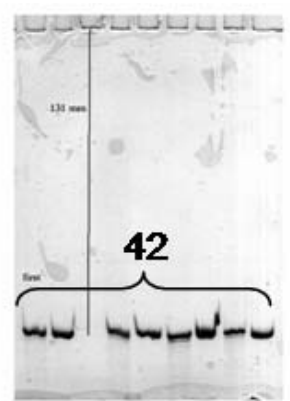

D

Figure 2.10: Array of gels comparing purity of synthesized DNA before and after PAGE purification. $1 \mu \mathrm{g}$ of DNA was loaded per well. Adjacent wells have purified and raw samples of the same strand. Numbers above each set of brackets refer the length in bases of the purified strands. All gels were denaturing PAGE: "A," "B," "C, "D," "E," and "F" were $20 \%$ gels. "G" was $11 \%$. Gels were run at $50^{\circ} \mathrm{C}$ at $500 \mathrm{~V}$. Run times were adjusted to allow appropriate resolution of bands. These results show all the strands had been adequately purified. 


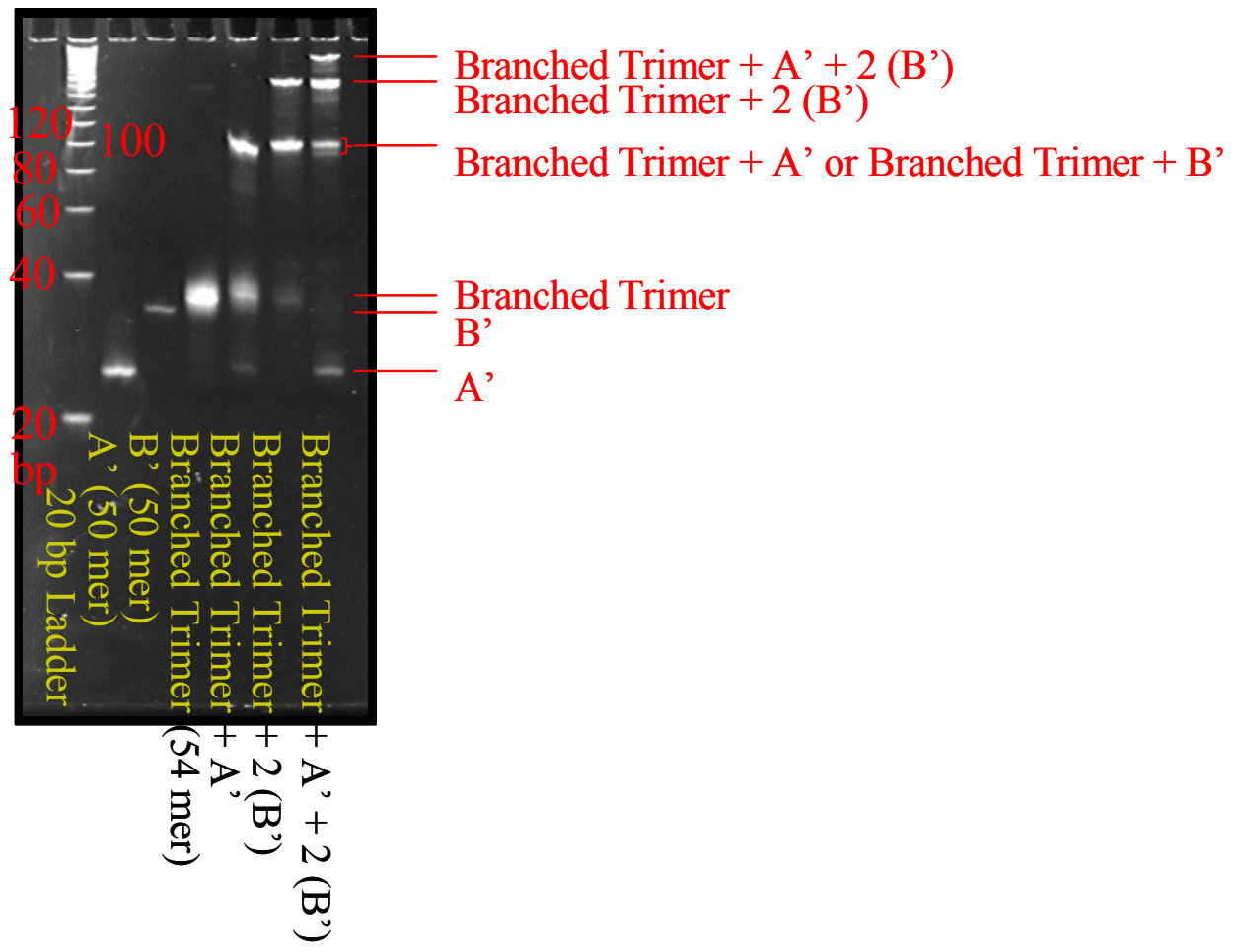

Figure 2.11: 12\% native PAGE gel showing proper double helix formation by an unusual three-branched DNA strand. The right-most three lanes show higher, more retarded, bands that correspond to addition of complementary DNA to the structure through duplex formation. This $16 \mathrm{~cm}$ gel was run at $4^{\circ} \mathrm{C}$ at $160 \mathrm{~V}$ in $1 \mathrm{X}$ TBE for $\sim 5 \mathrm{hrs}$. 
PAGE gel. This gel confirms the proper function of an artificially branched synthetic piece of DNA with three arms each of 18 bases. Two of the arms are the same DNA sequence, and are complemented by the 50 mer, B'. The third arm is unique, and is complemented by the 50 mer, A'.17 The DNA ladder loaded in the first lane is a double-stranded ladder in increments of 20 base pairs.

\section{Agarose gel electrophoresis}

PAGE is a useful electrophoresis medium, but it has relatively small pores. In order to sort larger things by size, we needed a support medium that could form bigger pore sizes. We used agarose gel to make gels with larger pore sizes. Agarose is a polymer component of agar, which is a non-toxic product of seaweed. In general, it gives gels with larger pore sizes than polyacrylamide. Agarose gels are made by dissolving agarose in buffer solution by heating, and then allowing it to cool to form a gel. Most agarose gels are run as a horizontal slab, as shown in Figure 2.12. Agarose gels can separate DNA in a similar manner to native PAGE gels as shown in Figure 2.13, but they lend themselves best to separations of long DNA (such as that from natural sources), or of large proteins. For DNA, we used Metaphor agarose. Metaphor agarose is a proprietary variation of agarose that has been optimized to separate short DNA strands like the ones we used in experiments in Chapters 3 and $4 .{ }^{18}$ One advantage of agarose electrophoresis of DNA is that the nucleic acid stain can be added to the gel solution itself before it has gelled (as we did for Figure 2.13), to allow us to monitor the DNA's progress through the gel using a handheld UV

\footnotetext{
${ }^{17}$ The sequence of this trimer is $\left(5^{\prime} \rightarrow 3^{\prime}\right): 2 \mathrm{X}($ GAT GGT CCT TGG TGG CGG)-(branch)-(GTC ACA TAG CGT TAC TGC).

${ }^{18}$ A $5 \%$ Metaphor ${ }^{\circledR}$ agarose gel will resolve DNA of 20-130 base-pairs. Metaphor agarose was purchased from BioWhittaker Molecular Applications (now Cambrex Bio Science Rockland, Inc.).
} 


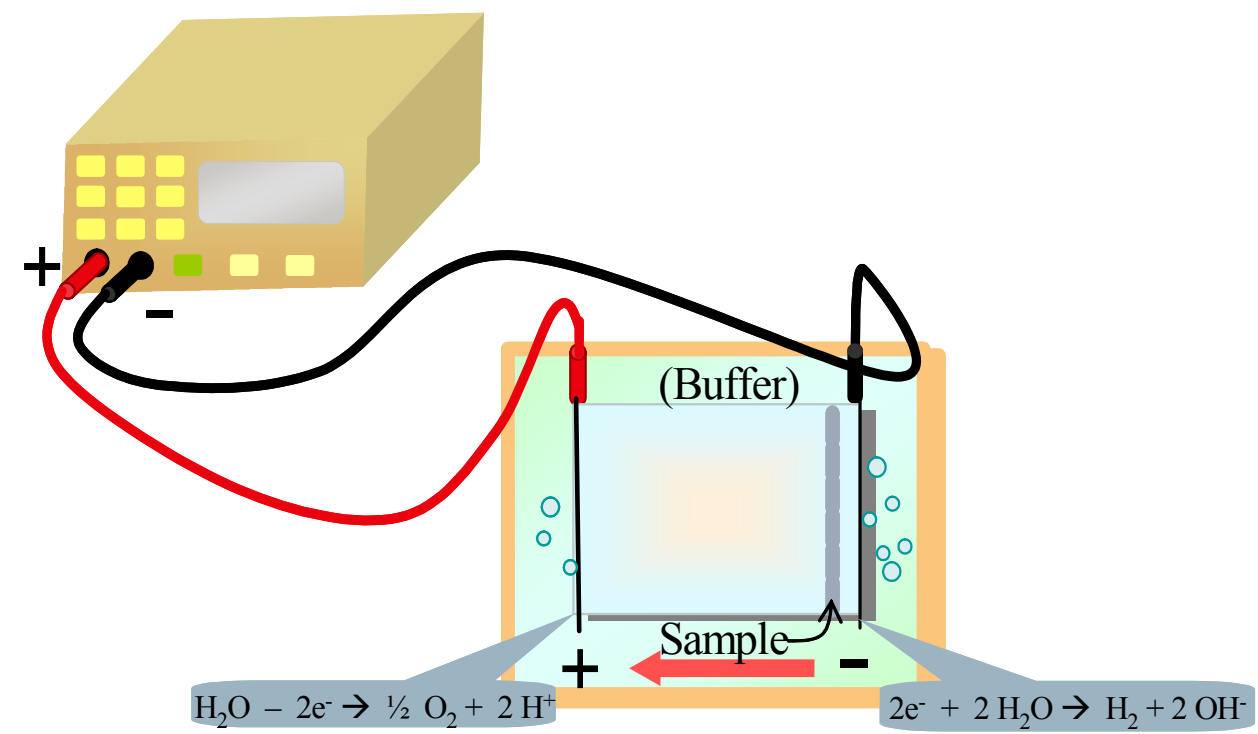

Figure 2.12: A diagram of a horizontal agarose gel electrophoresis setup, showing a gel submersed in buffer, and connected to a power supply.

lamp. In contrast, we cannot add this stain, ahead-of-time, to a polyacrylamide gel. The gel in Figure 2.13 tested the proper base-pairing of a short (18 base) dendrimer-labeled strand. It properly forms a duplex with its 50 base complement. (32 bases remain free and unpaired in this structure.)

We used agarose gel electrophoresis mostly to separate structures containing nanoparticles or nanocrystals. ${ }^{19}$ Nanoparticles are generally larger structures than DNA, and, provided they were both water-soluble and charged, they would move through the larger pores of agarose. We used the horizontal agarose and a preparative tube gel to perform separations of gold nanoparticle-containing structures. Figure 2.14 shows both of these types of apparatus. The tube gel provides a way to prepare larger quantities of a given separated sample, but its resolution capabilities are lower than the horizontal slab gels.

\footnotetext{
${ }^{19}$ We purchased most of the standard agarose as UltraPure ${ }^{\mathrm{TM}}$ Agarose from Invitrogen Life Technologies. Other suppliers were occasionally substituted, and the separations were similar.
} 


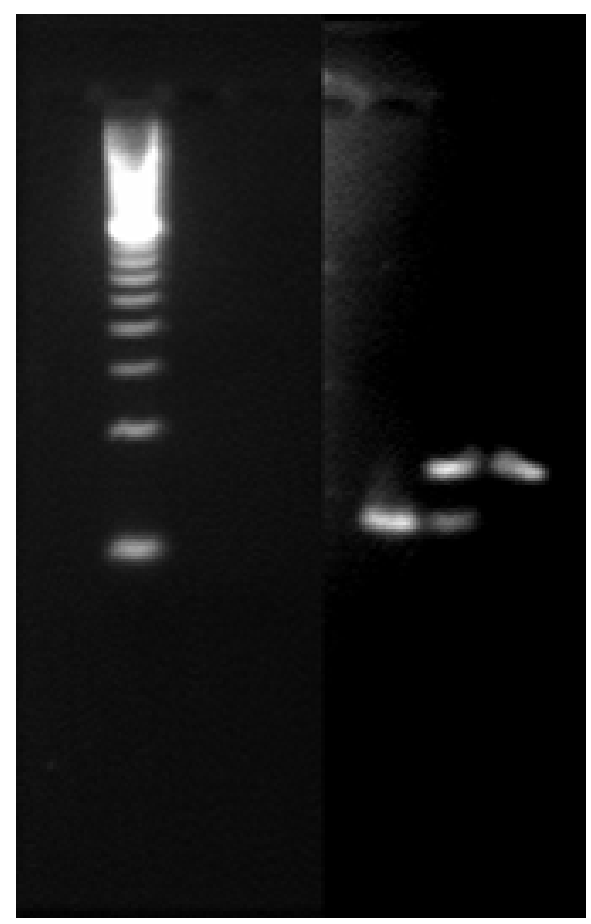

Figure 2.13: An agarose DNA separation gel. This native 5\% "Metaphor" agarose gel was run at $75 \mathrm{~V}$ for $2 \mathrm{hr}$. Lanes contain, left to right, a $20 \mathrm{bp}$ ladder, a 50 mer, the 50 mer + an 18 base complement, and the 50 mer + the 18 mer with an attached second-generation dendrimer. 

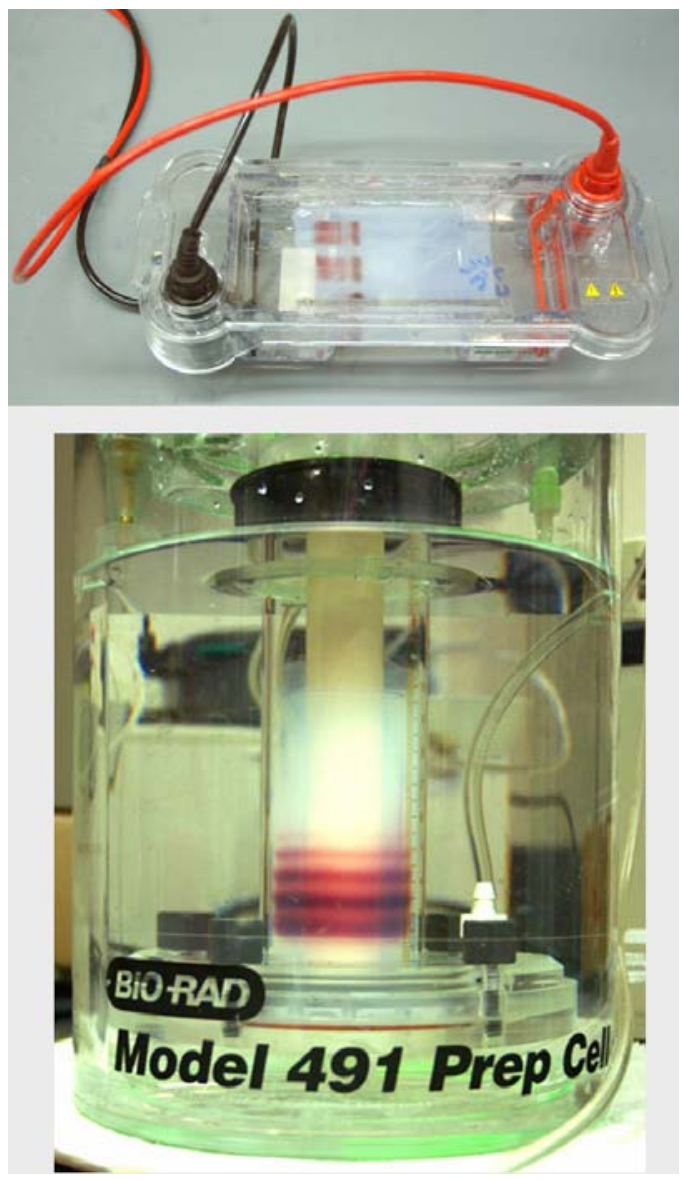

Figure 2.14: Two types of agarose electrophoresis units with gold nanoparticle samples. The top image shows a horizontal agarose gel box, and the bottom shows a preparative tube gel. 


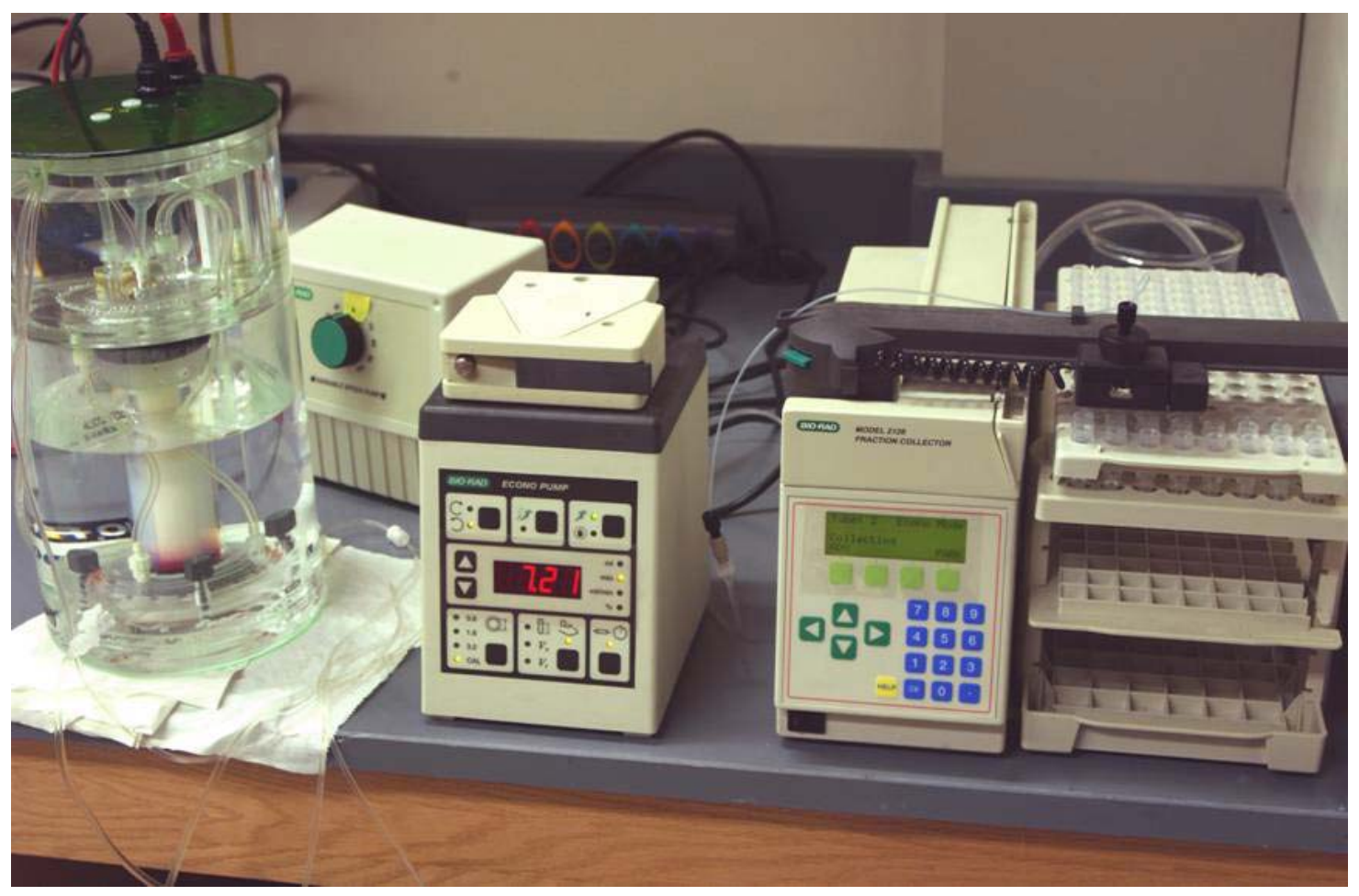

Figure 2.15: The entire preparative gel setup, with the attached fraction collector and pump, and an additional pump that provides cooling. (Purchased from Bio-Rad).

The tube gel setup is connected to a fractionator that collects the sample as it runs off the end of the gel, as Figure 2.15 shows. ${ }^{20} \quad$ It has the potential to make sample collection from the gel much easier, but for the experiments described here, it was unsuccessful in providing the necessary resolution of adjacent bands in collected fractions. Perhaps, with much greater effort toward the optimization of running conditions, it might be possible to make this setup useful. It could drastically reduce the labor costs for all of the experiments described here, as well as avoid providing aggregation conditions for the nanoparticles.

Agarose electrophoresis worked well to separate nanoparticle structures because the technology does a good job of separating large globular proteins. Figure 2.16 demon-

\footnotetext{
${ }^{20}$ The preparative tube gel we used was the Bio-Rad Model 491 Prep Cell attached to a Model 2128 Fraction Collector.
} 


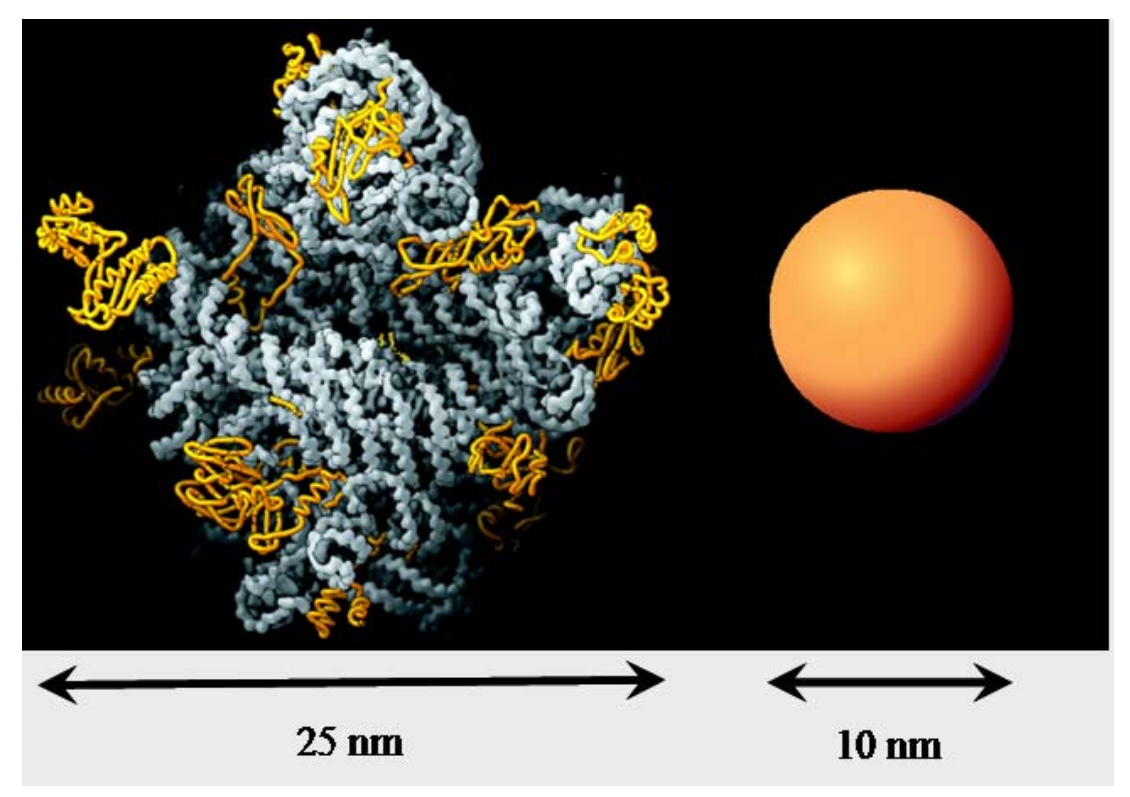

Figure 2.16: Shown on the left, the large ribosomal subunit, composed of RNA and protein, from the prokaryote, Haloarcula marismortui, [9] which is approximately $25 \mathrm{~nm}$ across; on the right, a proportionately scaled $10 \mathrm{~nm}$ gold nanoparticle.

strates the potential for an analogy between nanoparticles and proteins. Because of the comparable size and charge properties of nanoparticles and proteins, we were able to use protein techniques to separate different types of nanocrystal and nanoparticle structures. Agarose gel electrophoresis, for instance, distinguished 5 and $10 \mathrm{~nm}$ gold particles in the same way as it would distinguish proteins of different sizes, as shown in Figure 2.17.

The migration of the nanoparticles was largely governed by the type of charge present on the nanoparticle surface. Figure 2.18 shows the migration of yellow silanized semiconductor nanocrystals. During the silanization process, either amino or thiol groups, or both, have been added to the nanocrystal surface. The amino groups would be expected to have a $p K_{a} \sim 9 \longleftrightarrow 10,[40$, p.965] so they carry a positive charge at $p H \sim 8.3$ in a TBE buffer. For the samples with fewer or no amino groups, residual charge from unquenched 


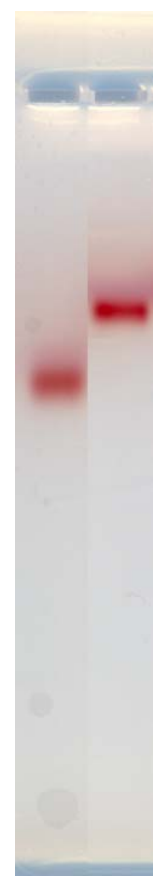

Figure 2.17: Spliced image of a single $10 \mathrm{~cm}$ agarose gel showing the migration of $5 \mathrm{~nm}$ gold compared to $10 \mathrm{~nm}$ gold coated with phosphine ligand.

silanol on the nanocrystal's surface may be the source of the negative charges that govern its movement toward the positive electrode.

\subsubsection{MALDI}

In order to be confident of our results, we needed to know that the DNA we used was pure. One way of characterizing the purity of DNA that we already discussed in Section 2.3.2 was to use a denaturing PAGE gel. Another method we also used was Matrix-Assisted Laser Desorption Ionization (MALDI). This method vaporizes and ionizes non-volatile biological samples that are in a solid phase to a gas phase, and then transfers them into a time-of-flight mass spectrometer, where they are separated by their mass-tocharge ratio. The mass spectrometer detects the ions at the end of a tube, and their flight 


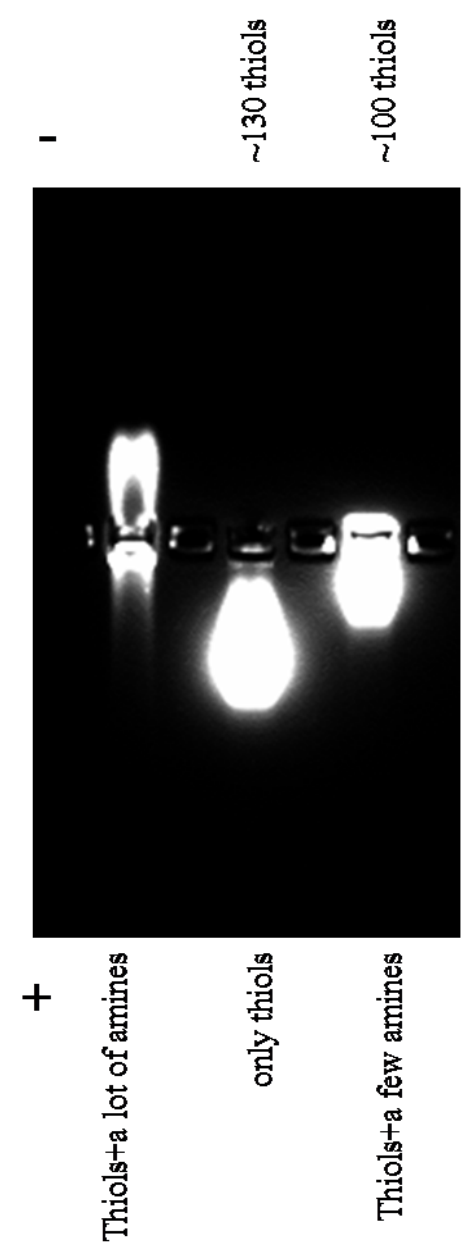

Figure 2.18: Three samples of yellow core/shell CdSe/ZnS nanocrystals coated with a siloxane layer, as described in Section 1.1.3. From left to right, the first lane contains nanocrystals that were primed with mercaptopropylsilane (MPS), then dialyzed against basified methanol, then subsequently coated with polyethylene glycol and aminopropylsilane (APS). The second lane had only MPS in place of the APS, and the third lane had a blend of $20 \%$ APS and $80 \%$ MPS. The agarose gel was run in $0.5 \mathrm{X}$ TBE at $100 \mathrm{~V}$ for 45 min by Dr. Daniele Gerion. 


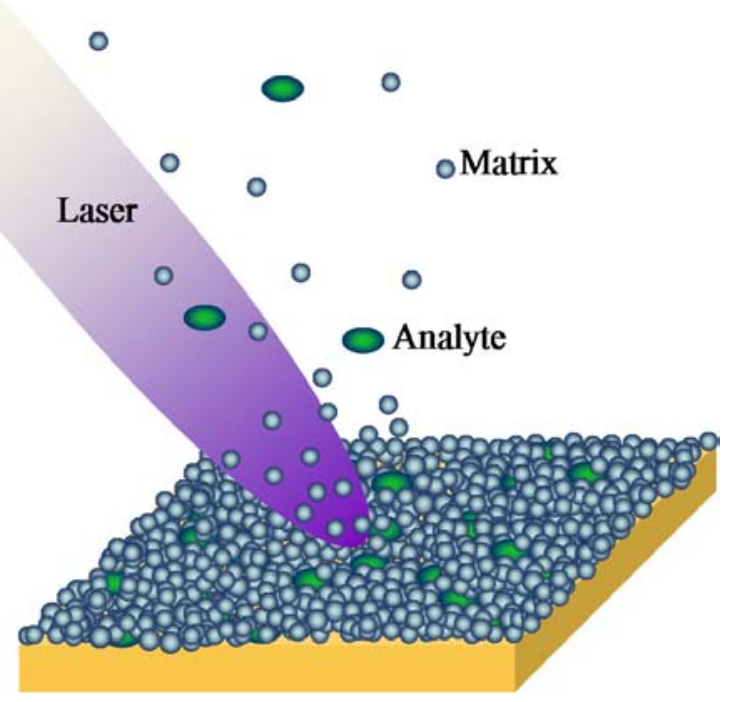

Figure 2.19: A laser pulse impacts the surface where the DNA analyte and the matrix have co-crystallized for MALDI analysis. (After a figure from srsmaldi.com.)[10]

time in the tube is proportional to $\sqrt{\frac{m}{z}}$. [10] For MALDI, we desalted a sample of DNA, replacing any sodium ions with ammonium, and mixed a solution of it with the matrix, 3-hydroxypicolinic acid (HPA). We dried about $1 \mu \mathrm{l}$ of the resulting solution onto a sample plate. The MALDI instrument directs a pulsed nitrogen laser onto the sample, as shown in Figure 2.19. The matrix absorbs most of the laser energy, largely reducing any sample damage and ion fragmentation. The choice of matrix is an empirically derived protocol for longer DNA strands. ${ }^{21}$ The resulting spectrum would allow us to determine the molecular weight of our DNA product, and confirm its likely purity by the absence of other peaks besides the main one. An example of this sort of MALDI spectrum is shown in Figure 2.20. Impurities of N-1, N-2, etc. DNA strands (not noticeably present here) would show

\footnotetext{
${ }^{21}$ For sample preparation, $19.5 \mathrm{mg} \mathrm{HPA}$ and $4 \mathrm{mg}$ of ammonium citrate were dissolved into $100 \mu \mathrm{l}$ of $\mathrm{CH}_{3} \mathrm{CN}$ and $100 \mu \mathrm{l}$ of water to make the matrix solution. $1 \mu \mathrm{l}$ of the matrix solution was dried on a sample plate, and then $1 \mu \mathrm{l}$ of DNA analyte was vacuum-dried on top of that for $30 \mathrm{~min}$.
} 


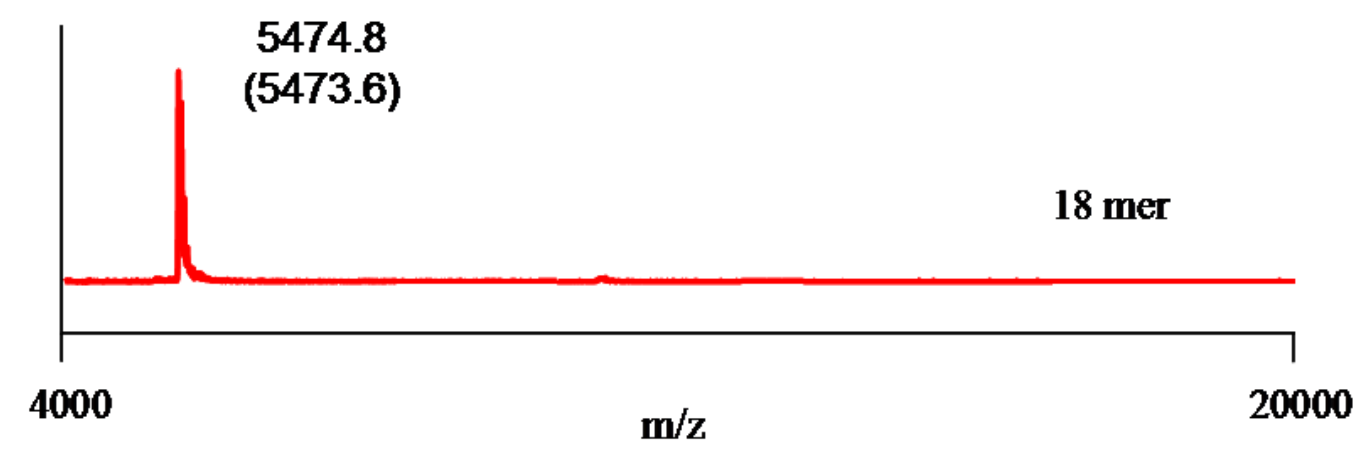

Figure 2.20: Observed MALDI spectrum for 18 base linear DNA. The top number is the resulting molecular weight. The expected molecular weight for the molecule is shown in parentheses. The result is within acceptable error for the instrument.

up as a shoulder on the low-mass side of the main peak.

\subsubsection{Unnatural synthetic modifications of DNA}

In order to attach DNA to other things, we need to be able to attach a reactive binding chemical group. Chemical synthesis of the DNA strands allows us to modify the strand with completely artificial functional groups. During the synthesis process, it is possible to incorporate a variety of linker groups or labels. These groups can add to the strand on either end, or even on an internal base.

\section{Thiol modification}

For the experiments described here, we ordered thiol-modified DNA from Integrated DNA Technologies. Thiol groups were added to the 5' or 3' end of the DNA strand according to the needs of the experiment.

One method of 5' thiol modification added (S-trityl-6-mercaptohexyl)-(2-cyano- 
ethyl)- (N,N-diisopropyl)-phosphoramidite to the strand while it was still on the automated synthesizer. The thiol's trityl group does not come off during the normal deprotection conditions; instead, it is removed by oxidation with silver nitrate. Dithiothreitol (DTT) precipitates excess silver nitrate, and the DTT comes out during desalting.[64, p. 25]

Another method for adding 5' thiol modification used 1-o-dimethoxytrityl-hexyldisulfide, 1'-[(2-cyanoethyl)-(N,N-diisopropyl)]-phosphoramidite. The disulfide was cleaved with DTT, and then the DNA was desalted to remove the DTT. We used this method of modification most often.[64, pp.26-27]

3' thiol-modified strands were grown off 1-o-dimethoxytrityl-propyl-disulfide, 1'succinoyl-long chain alkylamino-controlled pore glass as a solid support. DTT added during the final deprotection stage cleaved the DNA from solid support having a free thiol. Desalting removed excess DTT.[64, p.55]

\section{Synthetic branching}

For the DNA trimer-based project described in Chapter 3, we synthesized DNA with a branched polymer backbone. Doubling and trebling synthons provide two or three synthesis points in a DNA sequence where there was formerly only one.[65][11] We synthesized DNA dendrimers using the now commercially available doubling reagent shown in Figure 2.21. DNA was synthesized, starting at the 3' end, in the normal manner off of the $1000 \AA$ solid support for a length of 16 to 20 bases. We then added the doubling synthon using the automatic synthesizer. The doubling synthon coupled at high yield, comparable to that of the other bases. Synthesis afterwards proceeded off of the doubling synthon's two free hydroxyl groups (after deprotection). We needed only a small amount to perform 


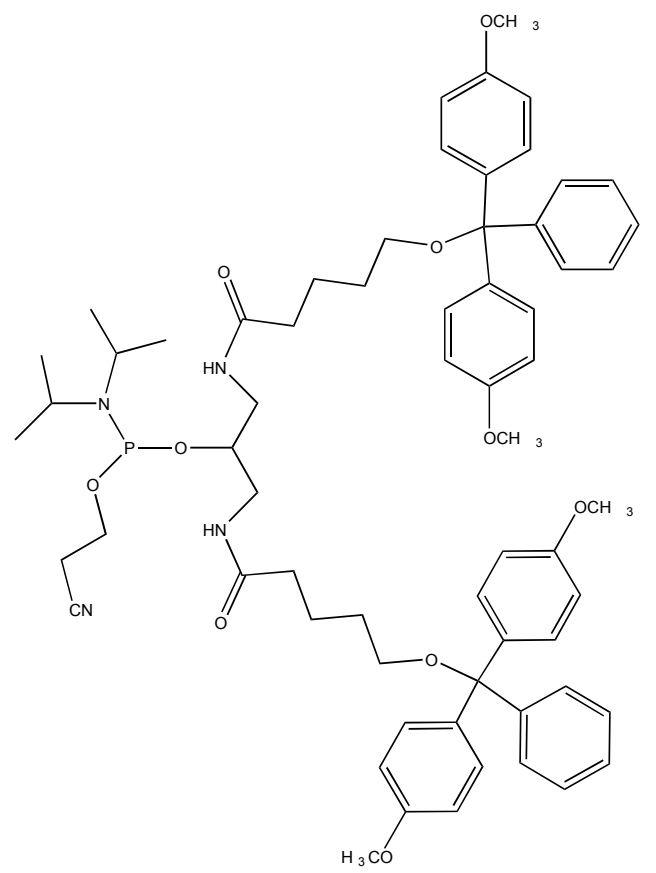

Figure 2.21: Doubling synthon phosphoramidite purchased from Glen Research, as described in the 1999 Nucleic Acids Research paper by Southern, et. al.[11]

the experiments in described in Chapter 3. 


\section{Chapter 3}

\section{Using DNA Trimers to Arrange}

\section{Gold Nanocrystals}

\subsection{Motivation}

One of the main goals of this research project was to produce discrete, defined, nanostructures that contain nanocrystals in a specific configuration. This idea can be imagined as a scaling-up of chemical synthesis, where atoms are the building blocks that make molecules. In the scaling up, atoms are turned conceptually into nanocrystals or particles, and the bonds become the DNA molecules.[46] Previous research has shown that gold nanocrystals can be arranged in specific, discrete structures of two or three particles, as shown in Figure 3.1.[46][1] To make the top two structures, Colin Loweth added 0.9 equivalents of single-stranded DNA to 5 or $10 \mathrm{~nm}$ gold nanoparticles coated with a charged 

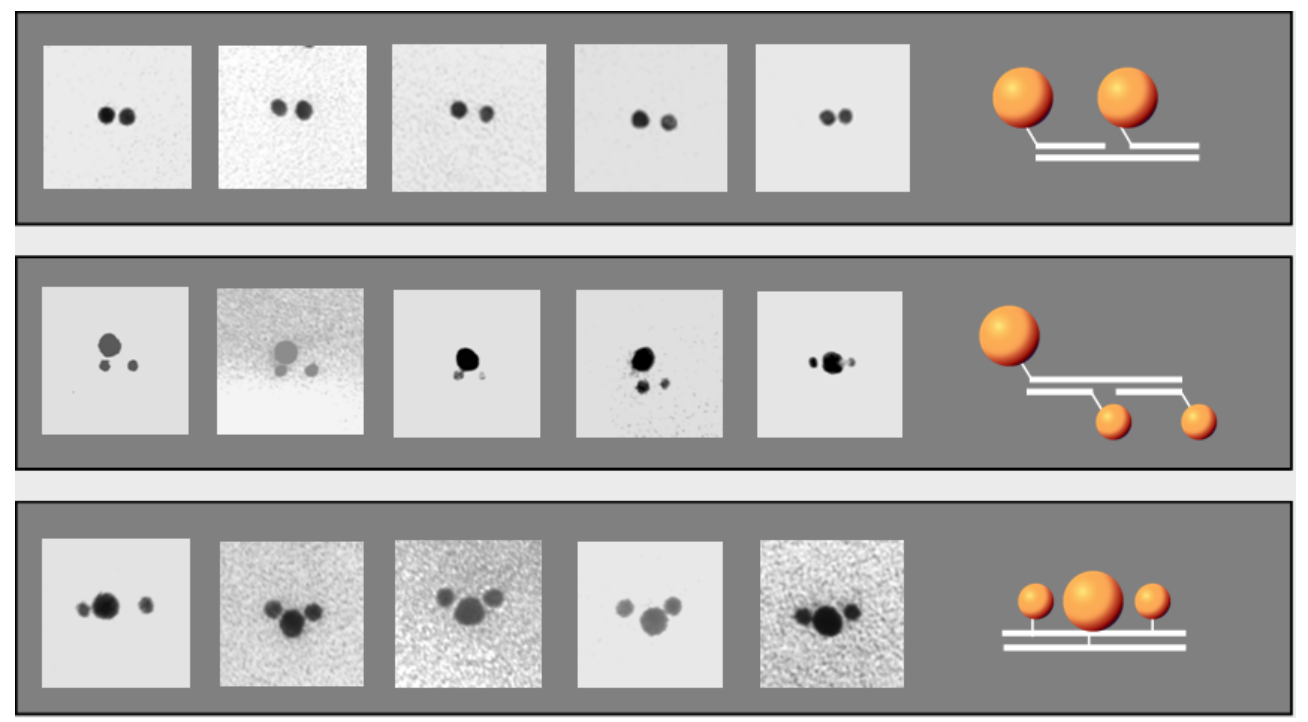

Figure 3.1: DNA links two sizes of gold nanoparticles into discrete structures visible under TEM. (Results from [1].)

phosphine ligand. ${ }^{1}$ He hybridized the gold-labeled DNA strands with their appropriate complement, either labeled or unlabeled, for a period of 1 to 4 days, and then isolated the resulting structures from $3 \%$ agarose gels. The bottom structure had an additional step (not shown), where he first hybridized the doubly-thiolated DNA strand with its complement, and then added the gold nanoparticles, because the stiffness of the resulting double helix averted the possibility of making a double-thiol attachment with single gold particle. After isolating this structure, he melted the complement away and then treated with the new gold-labeled complement to give a heterotrimer structure.

Figure 3.2 shows our own initial target structure using the branched synthetic DNA trimer. We synthesized the asymmetric branched DNA trimer according the procedure

\footnotetext{
${ }^{1}$ We purchased gold nanoparticles in 5 and $10 \mathrm{~nm}$ sizes from Ted Pella, Inc (Redding, CA). Dr. Loweth used the same source. These particles were coated in bis(p-sulfonatylphenyl) phenylphosphine dihydrate, dipotassium salt from Strem Chemicals (Newburyport, MA) according to previously the reported procedure.[1]
} 


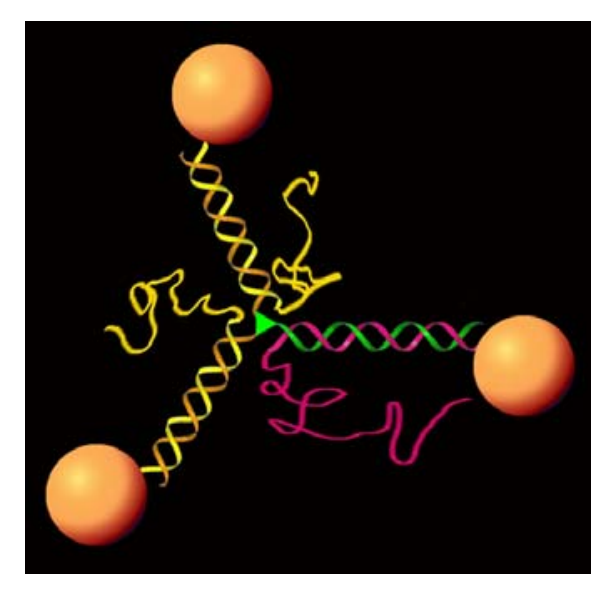

Figure 3.2: Cartoon showing the design features for the target gold trimer structure containing an asymmetric, branched, DNA trimer. The identity of DNA sequence is keyed to color. Two arms of the structure contain the same sequences. The third arm is unique.

described in Chapter 2.3.4. Attaching gold to the structure required two distinct kinds of DNA-Au conjugates. The DNA for one type presented a thiol group at its 5 ' end. The other presented its thiol at the 3' end. This arrangement resulted from our method of synthesizing the DNA trimer. The trimer was not synthesized in three parts, and then joined in the middle, post-synthesis, because this procedure had a low yield as when it was tried by Young-Wook Jun. The trimer was instead synthesized on solid support, with a branch point introduced during synthesis, so that the DNA trimer had two 5' ends and one 3' end. The complements, since the DNA double helix is antiparallel, required thiols on the end furthest from the trimer's central branch point. Thus, twice as many 3' DNA-Au conjugates were needed for the experiment as 5 ' conjugates.

The structure, as pictured in Figure 3.2, has un-base-paired segments that form a tail on each conjugate. This tail was added to the initial design because the process for creating these conjugates was slightly different from that used by Dr. Loweth. First 


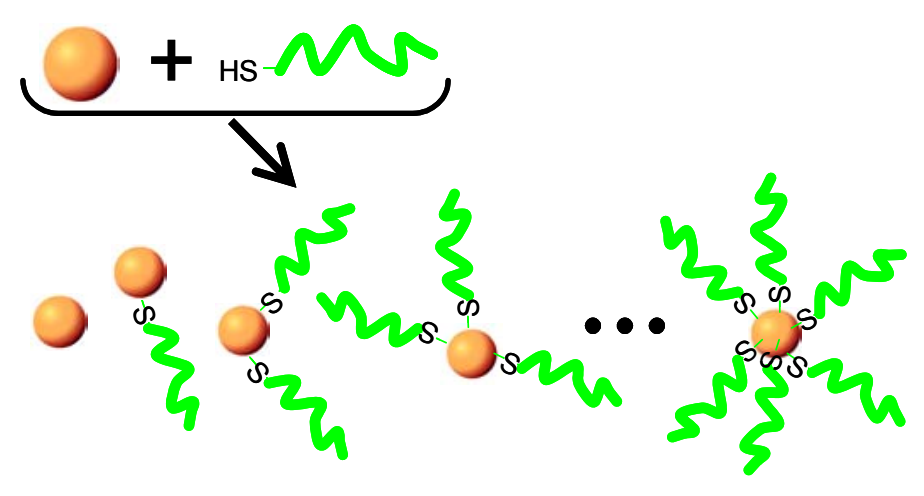

Figure 3.3: Addition of thiolated DNA to gold nanoparticles yields a mixture of reaction products that have dispersity in the number of DNA strands added to the gold. The number present of any particular type of conjugate depends on the stoichiometry of the DNA/Au ratio.

discrete DNA-Au conjugates were isolated according to published procedures.[66] Addition of DNA to gold nanoparticles produces a mixture of reaction products, as depicted in Figure 3.3. If the unpurified products of this reaction mixture are added to their similarlyprepared complementary conjugate, uncontrolled aggregation will result. Figure 3.4 shows this conceptual problem of producing a defined structure under these circumstances. In order to avoid uncontrolled aggregation, it is necessary to isolate discrete gold nanoparticle conjugates with a discrete, countable number of DNA. Provided the DNA is long enough, it will retard the mobility of the gold nanoparticles compared to that of the free phosphinecoated gold. Figure 3.5 shows a gel that demonstrates this effect. To prepare conjugates such as these, the proper stoichiometric ratios were mixed in the presence of $50 \mathrm{mM} \mathrm{NaCl}$ and allowed to stand at room temperature for two hours. Leaving the conjugation reaction for longer than about three hours resulted in either very fuzzy or totally indiscernible bands in an agarose gel prepared like the one in Figure 3.5. While the gel electrophoresis of DNA$\mathrm{Au}$ conjugates had to happen promptly in order to isolate the discrete bands, the resulting 


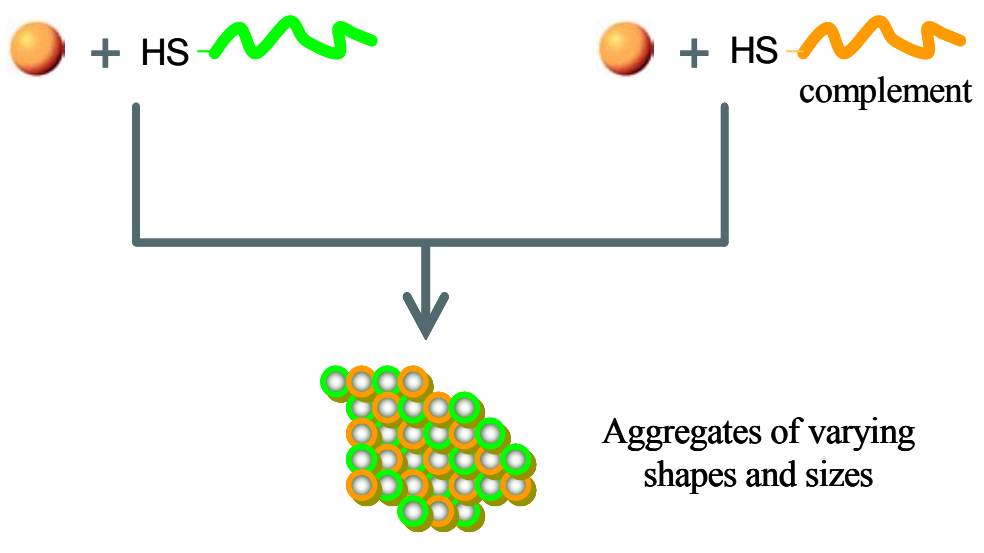

Figure 3.4: Addition of the unpurified reaction products from Au-DNA conjugate preparations of complementary DNA strands will result in uncontrolled aggregation. The resulting structures will be clumps of gold particles without specific order, regardless of the claims of Prof. Mirkin and others.[12]

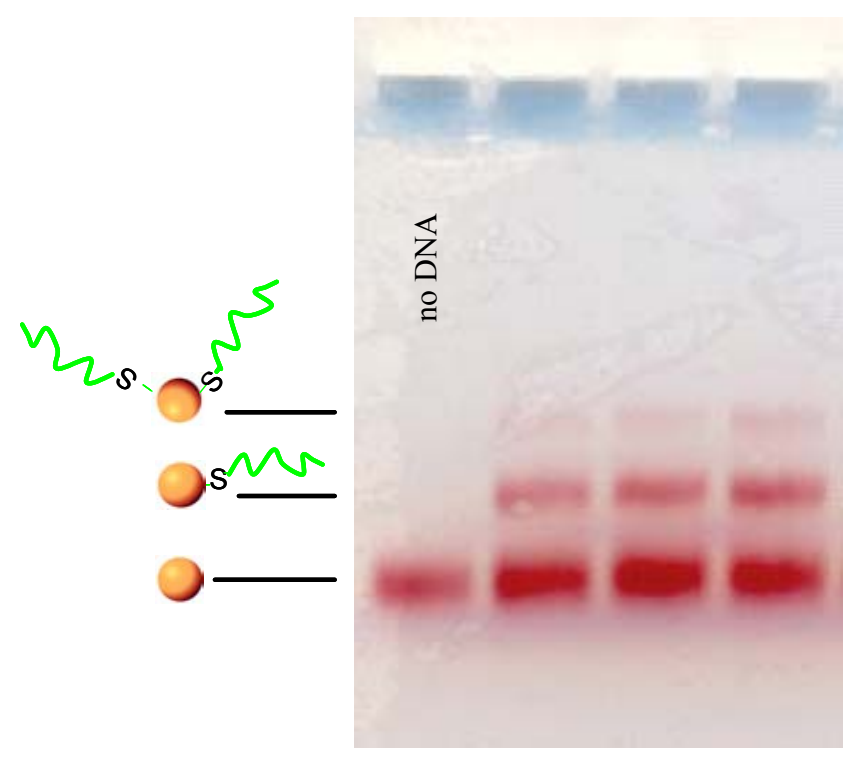

Figure 3.5: Titration of 100 base thiolated DNA addition to $10 \mathrm{~nm}$ gold particles. The leftmost lane has 2 pmol of phosphine-coated gold sample. The next three lanes have 2:1, $3: 1$, and 4:1 ratios, respectively, of DNA to gold. The resulting bands in the gel are labeled according to the type of conjugate they contain. The efficiency of DNA addition to gold varied widely from sample to sample. It was not possible to quantitate the DNA on the gold by UV/Vis spectroscopy because the gold itself absorbs heavily at the relevant $260 \mathrm{~nm}$ DNA base peak. (See Figure 2.1.) Poor DNA or gold samples led to more diffuse bands in the agarose gel. 
single or double conjugate sample would last some weeks in a phosphine-containing storage solution unperturbed, provided the samples were not concentrated to the point that they irreversibly aggregated. Aggregated samples had a bluish-purple tinge, and the entire gold content of a vial would settle to the bottom in a blue clump eventually, after the point of attaining a bluish tinge to the sample. Such samples were irrecoverable and had to be discarded. Discrete conjugates were discernible for 'good' gold and DNA samples (where the DNA was also at least 50 bases long). There were occasional examples of DNA shorter than 50 bases providing discrete bands in a gel, but strands shorter than 50 bases pushed the limit of band resolution under these separation conditions. Our gold trimer design contained gold/DNA conjugates with DNA 50 bases in length for this reason. 100 base DNA certainly gave better results for band resolution, but it was not possible to purchase 3'-thiolated DNA having more than 50 bases. Our attempts to synthesize this longer DNA with 3' thiols on our own failed, perhaps because of our inability to completely extract all of the remaining DTT remaining from the disulfide cleavage reaction. This problem limited our success on the overall project.

The synthesis of the branched DNA trimer was also length-limited as well, but in the opposite sense. The reaction yield of two strands after the branch point in the strand became unreliable, and any error in either strand ruined the product, increasing the DNA trimer production failure rate. DNA trimers longer than 20 bases in an arm were extremely low yield, as was a trimer synthesized in the opposite direction with nonstandard reagents(5' to 3'). The DNA trimer in Figure 3.2 therefore has arms of 18 bases, and complementary conjugates with 50 bases apiece. 


\subsection{Structure and Sequence Design}

Successful DNA nanoassembly requires meticulously prepared DNA sequences. The sequence of the DNA must be structured so that its thermodynamic equilibrium structure is the one that is most desired. Any given piece of DNA has a library of possible associations and configurations competing for it. If the desired structure is not the one most thermodynamically favorable under the preparation conditions for the experiment, that structure will be rare, at best. More likely, it will be nonexistent among the end reaction products. Using known thermodynamic variables to calculate the most thermodynamically favored configuration for a set of DNA strands is a challenging statistical mechanics prob-

lem, and one tackled by numerous careers in the related field of biologically-oriented RNA research. For these experiments, no such DNA program was known to generate reliable results, but it was possible to generate a set of intuitive rules that describe the conditions under which a DNA sequence would be expected to perform in the desired manner.

In this way of thinking, we consider every DNA strand to consist of a series of overlapping units of a few bases per unit. The length of the unit is a parameter we set, roughly speaking, as the shortest length of bases that we require to be unique within the structure. Prof. Nadrian Seeman (now at New York University) developed this method in the 1980's to create a program he called "Sequin" that he (and we) used to design DNA sequences for various structures.[67][13][68][69] It uses a number of rules to ensure that the desired structure has the maximum amount of Watson-Crick base-pairing over any wouldbe competing configurations. The user selects the desired architecture for the structure, and then either she or the program can select the sequence, with concurrent checking for 
possible competing configurations of the strands. The underlying premise of the program is that DNA will, all things being equal, prefer to be in a continuous Watson-Crick basepaired double helix. The "vocabulary" of elements that we have to design this sequence for DNA is limited by nature to four: the four bases A, T, G, and C. However, if we required every base in a sequence we were designing to be unique, we would be limited to a strand of merely two bases, together with its complement, for a total of four bases used! Obviously, a longer strand of DNA must require less stringency for its uniqueness. If we allow ourselves to repeat bases in the sequence, provided all two-base permutations are unique, we give ourselves more freedom. Now there are sixteen possibilities, AA, AT, AG, AC, TA, TT, TG, TC, GA, GT, GG, GC, CA, CT, CG, and CC. (Order matters because of the directionality and asymmetry of the polymer backbone in the DNA strand. For instance, "AT," is not the same as "TA," read in a $5^{\prime} \rightarrow 3^{\prime}$ direction.) If we require all two-base permutations in the strand to be unique, we can now design a double strand up to eight bases long. These two-base permutations are called "vocabulary elements" or "critons" by Seeman. Requiring that all three-base permutations be unique gives 64 possibilities, and so on. The larger the vocabulary element, the larger the structure that can be designed. The length of the vocabulary element specifies the maximum length of any competing interaction in the strand. What length of competing interactions in strands would be sufficient to create a significant source of competition in forming the end structure depends on the sequence itself, the DNA concentration during annealing, ambient temperature, and the type and amount of salt present in the solution. It is desirable that the melting temperature of the competing interactions be below the temperature of the 
experiment. In practice, a competing vocabulary element of six bases starts to provide a source of worry to a sequence designer. Our sequences were designed so that all fivebase overlapping units should be unique. A second-order problem occurs (and the program Sequin does not detect this one) when, say, a set of three bases is duplicated in the structure, then an unduplicated base follows, followed by a second set of three duplicated bases. The result of this setup is that there is a set of seven bases present in the strand where six of those seven have two different structural locations that have them hydrogen-bonded into a double helix. The vocabulary elements are unique, however, and the program does not directly protect against this problem as it stands, except by sliding the strands alongside each other, totalling all Watson-Crick interactions for each position along the slide (with the "MISMATCH" command). With this program, it is possible to create unpaired regions, and junctions, although we did not use this feature to design our branched DNA trimers. An overview of the structure of Sequin is shown in Figure 3.6. In step 1, the user chooses the desired architecture for the DNA structure. The user specifies the covalent backbone, and where base-pairing relationships are desired. In step 2, the computer ranks the locations in the structure where bases will be assigned to provide the most efficient method for computing the sequence. In step 3, either the user or the computer assigns the bases, and in step 4, the assigned sequence of bases is tested against the four rules (quoted from Prof. Seeman) below:[13]

1. Each criton [vocabulary element] in the individual strands forming the junction [the desired architecture] must be unique throughout all strands.

2. The complement to any criton that spans a bend in a strand must not be present in 


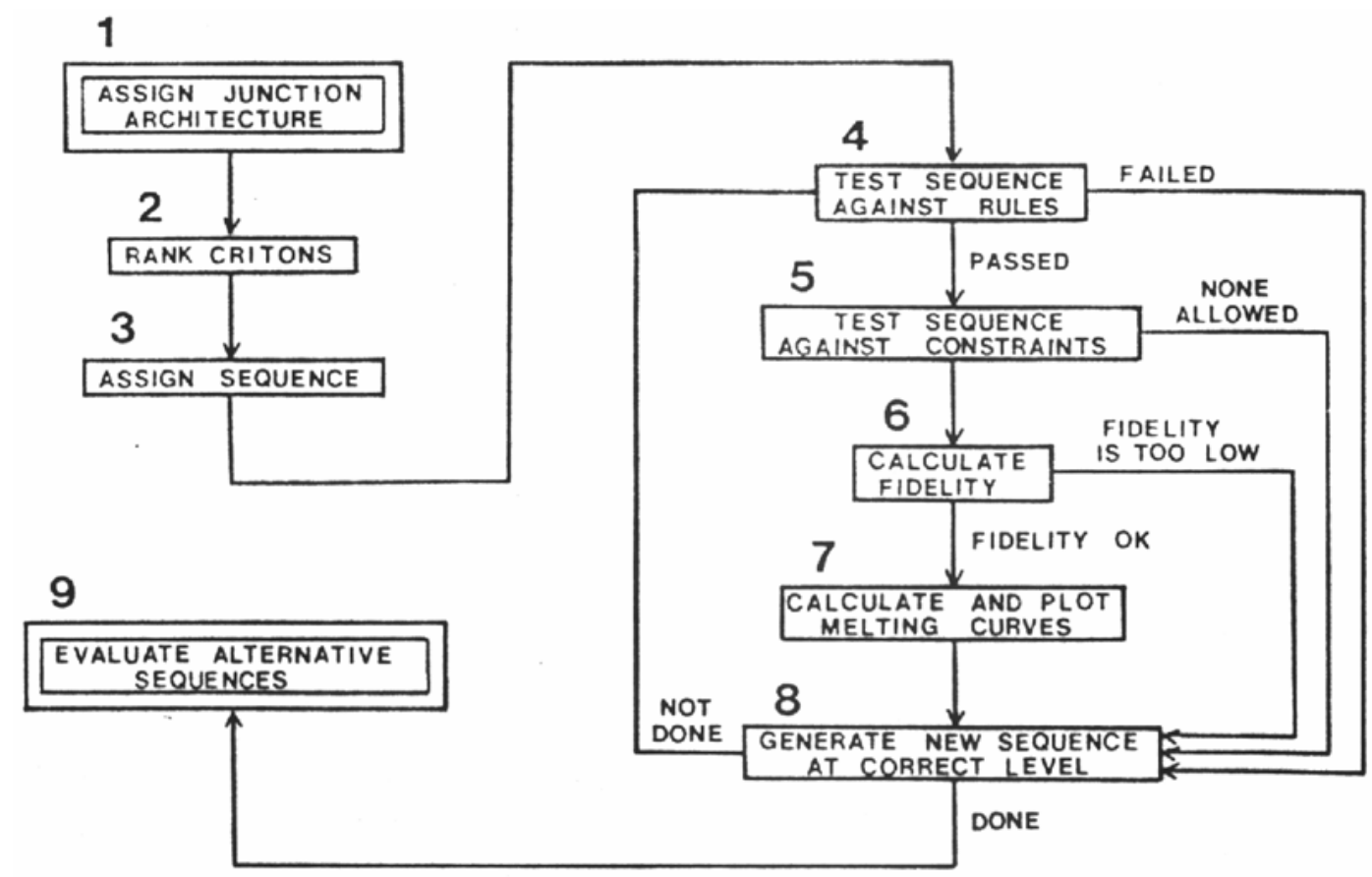

Figure 3.6: Flow chart describing the process contained in Prof. Nadrian Seeman's "Sequin" program.[13] 
any strand.

3. Self-complementary critons are not permitted. [If the criton length is an odd number, this statement holds true for all segments one longer than the criton length.]

4. The same base pair can only abut. . . [a] junction twice. If it is present twice, those two occurrences must be on adjacent arms.

In step 5 of the Sequin program, the sequence, if so far successful, is tested against any constraints the user imposes (for instance, to avoid possible non-Watson-Crick basepairing, or to require the ends of strands to contain G-C pairs to prevent easier end-fraying).

Steps 6 and 7 were designed to incorporate thermodynamic information into the testing. However, the parameters included in Sequin are incorrect, and Prof. Seeman expressed no confidence in the result, ${ }^{2}$ so we ignored these results. Step 8 generates a new sequence to compare to the first, and the whole cycle for steps 4 through 8 repeats until enough alternate sequences exist for the user to compare and select the best. In practice, because the thermodynamic values for the program were not correct, we only performed steps 4 and 5, and did not cycle through alternate sequences. There is plenty of room for improvement in this program. It would be useful to correct the thermodynamic evaluations of the sequences. Even better, but substantially harder to execute, would be to predict the conformation of a given strand given its sequence. Also the program itself is fifteen to twenty years old, and computer interfaces have improved substantially during the intervening period. In its current form, Sequin is difficult to use.

In order to design our DNA trimer sequences, we used Sequin to assign a sequence

\footnotetext{
${ }^{2}$ personal communication $(2001)$
} 
for a single, unbranched DNA double helix long enough to include all of the arms' longest sequences. We then chopped the long sequence into pieces corresponding to each trimer arm, removing any bases we didn't need (such as the unused complement to the singlestranded tails on the DNA that was attached to the gold). In this manner, we assigned sequences for four different DNA trimers, with hybridization properties that corresponded to the needs of the experiments.

\subsection{Nanocrystal-DNA Conjugate Preparation}

In order to prepare any of the structures based on a DNA trimer, it was necessary, first, to prepare conjugates having a single DNA strand per gold particle. As discussed in Section 3.1, DNA of a length of at least 50 bases gave discrete bands for each addition for 5 or $10 \mathrm{~nm}$ gold particles. In order to tailor the efficient preparation of conjugates, we performed titrations of the DNA strands with each gold sample to optimize the stoichiometry of the DNA reacted to the gold. Not all gold or DNA samples behaved the same way, or conjugated with the same efficiency. Figure 3.7 shows an example of a series of titrations of 50 base DNA with four different gold samples. ${ }^{3}$ After optimizing the stoichiometric ratios to give the highest yield of the desired conjugate, we scaled up the reaction as a large batch. The reaction batch was loaded into a series of gels containing identical wells at 40 pmol of loading per $6 \mathrm{~mm}$ well. Many gels were necessary to prepare a sufficient amount of gold/DNA conjugate. Figure 3.8 displays two gels designed to allow maximum preparation of a single DNA-gold conjugate. We isolated the desired single-DNA-strand conjugate from the gel

\footnotetext{
${ }^{3}$ The DNA sequences for this procedure were as follows: Sequence "A": 5'-CGG GTT CAA TTC GCA TTT GTA GTC TTG AGC CCG CAC GAA ACC TGG ACA GT-SH-3'; Sequence "B": 5'-GTG AAG TCG ATC ATG GAG TAC ATT GTC AGT TAG CTC GTT CGA GGA TGT TG-SH-3’.
} 


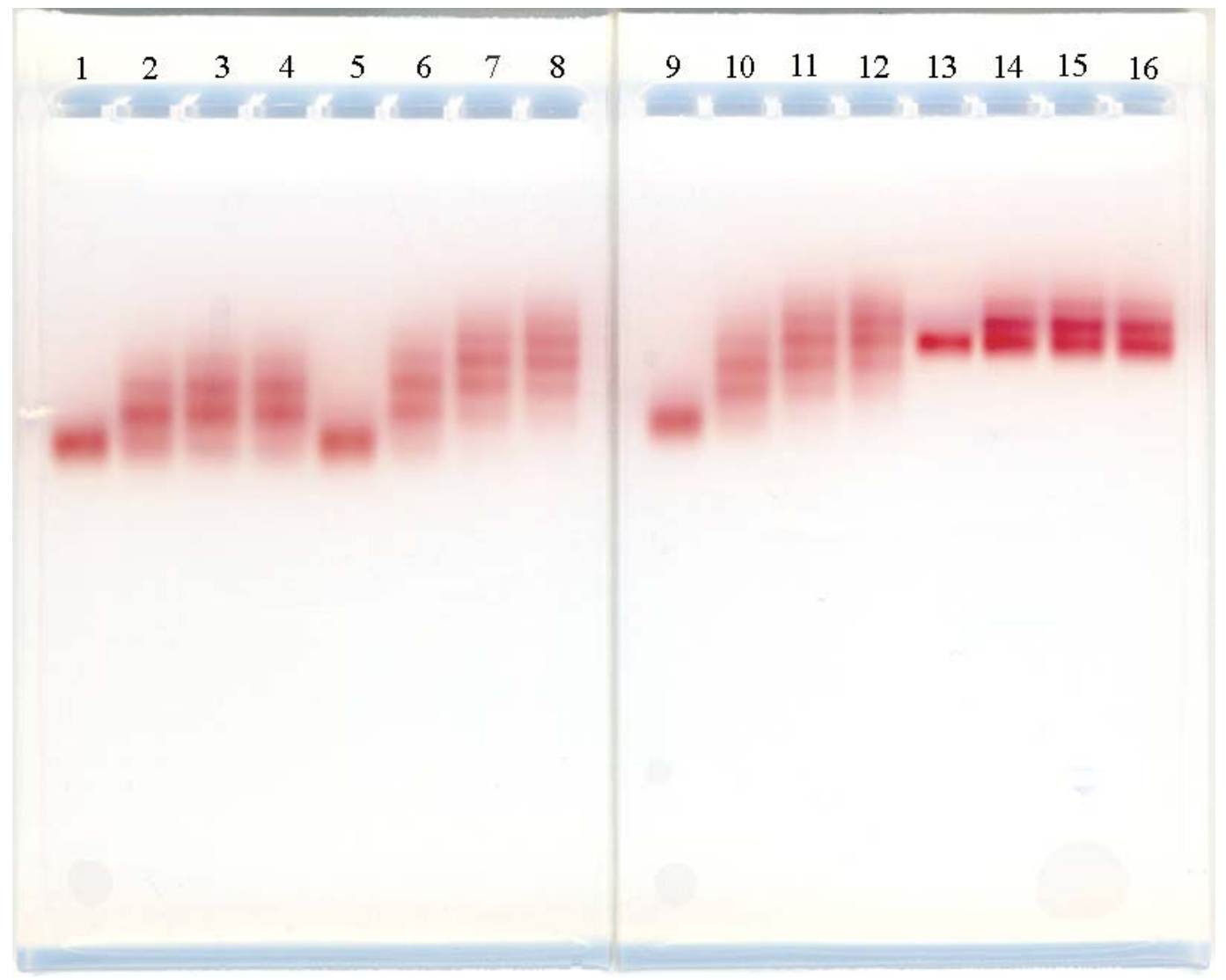

Figure 3.7: Series of four simultaneous conjugate titrations reacted for about two hours before gel loading. From left to right, lanes 1-4 show 50 base 3'-thiolated DNA sequence "A" added to $5 \mathrm{~nm}$ gold. Lane 1 is gold only, 2 has gold +0.33 eq. of DNA, 3 has gold +0.66 eq. of DNA, and 4 has gold +1 equivalent of DNA. Lanes 5-8 and 9-12 show addition of the same DNA to two other samples of gold, with lanes 5 and 9 having plain gold, and each successive lane having 1, 2 and 3 equivalents of DNA, respectively. The four rightmost lanes show addition of 50 base 3'-thiolated DNA sequence "B" to $10 \mathrm{~nm}$ gold. Lane 13 has unconjugated $10 \mathrm{~nm}$ gold, and lanes 14-16 have 1, 2, and 3 equivalents, of DNA respectively. Unmodified lanes were loaded with 2 pmol of $10 \mathrm{~nm}$ gold or 20 pmol of $5 \mathrm{~nm}$ gold. Conjugate lanes were loaded with $4 \mathrm{pmol}$ of $10 \mathrm{~nm}$ gold, or 35-40 pmol of $5 \mathrm{~nm}$ gold. Loading heavily impacts the visibility and resolution of bands. 


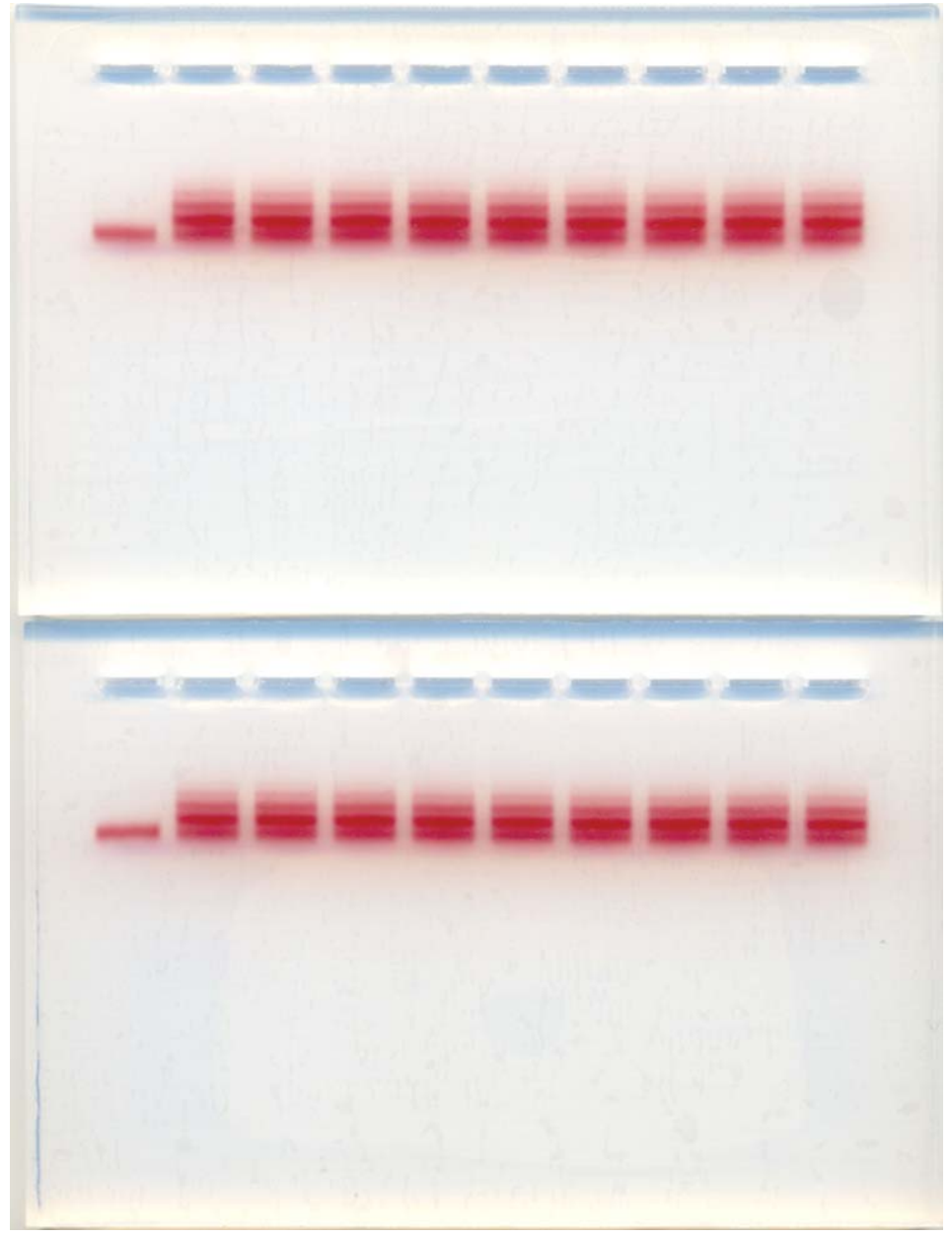

Figure 3.8: Two gels out of eight containing the products of a preparatory reaction of 10 nm gold nanoparticles with 50 base DNA (sequence "B"). ${ }^{3}$ 
by removing the buffer overlay on the gel, slicing the gel just beyond the band we wanted to collect, and inserted a lane-sized piece of filter paper backed by dialysis membrane. ${ }^{4}$ Then we electrophoresed the gold into the filter paper until the band was collected (6 to 10 minutes), and removed the filter paper/membrane combination from the gel. To collect the gold conjugate from the filter paper, we spun it out into centrifuge tubes. ${ }^{5}$

Although this process usually recovered at least some sample from the gel, it had several drawbacks. First the recovery from the gel was not high yield. In addition to the gold lost to conjugates with too few or too many DNA, gold was also lost during electrophoresis into the filter paper, while withdrawing the filter paper from the gel, and from incomplete recovery from the filter paper. Also, recovered samples had a significant chance of precipitating immediately after recovery. Although we considered alternate ways of recovering the samples, we never managed to make any of the alternatives work satisfactorily. An overview of this problem is evident in Figure 3.9, where an array of identical isolated samples is shown in both overhead and transilluminated views. We combined samples such as these into a single vial and condensed to give an opaque bloodred sample of the highest concentration we could manage without it aggregating. ${ }^{6}$

\footnotetext{
${ }^{4}$ The filter paper needed to have a pore smaller than the size of the nanocrystals. We generally used MWCO (molecular weight cutoff) membrane of 10,000 MW, although we occasionally substituted 8000 or 15,000 MW. We pre-soaked the membrane in the electrophoresis buffer for at least ten minutes to remove sodium azide preservative. The filter paper was Whatman GF/C. Another type tried did not work properly.

${ }^{5}$ We either placed the filter paper/membrane combination, filter paper down, into an Ultrafree-MC 0.22 $\mu \mathrm{m}$ centrifuge filter inside a $1.5 \mathrm{ml}$ centrifuge tube and spun it in a low-speed personal centrifuge to recover the sample, or we placed it into a $0.5 \mathrm{ml}$ centrifuge tube having a needle-punched bottom, and placed that tube inside a $1.5 \mathrm{ml}$ centrifuge tube and spun that tube combination in the centrifuge. The ultimate goal was to recover the sample, minus any filter paper or fibers, without precipitating it. Usually we added $10 \mu \mathrm{l}$ of fresh $40 \mathrm{mg} / 100 \mathrm{ml}$ phosphine solution to the filter paper before spinning to help retain the gold in solution.

${ }^{6}$ Generally, these samples were either 1.5-2 $\mu M$ in $5 \mathrm{~nm}$ Au conjugates, or $0.3 \mu M$ in $10 \mathrm{~nm}$ Au conjugates. Sometimes previously good samples aggregated completely during the extensive amount of centrifugation necessary to achieve these concentrations. We condensed the samples in Centricon 10,000-50,000 MWCO membrane filters.
} 

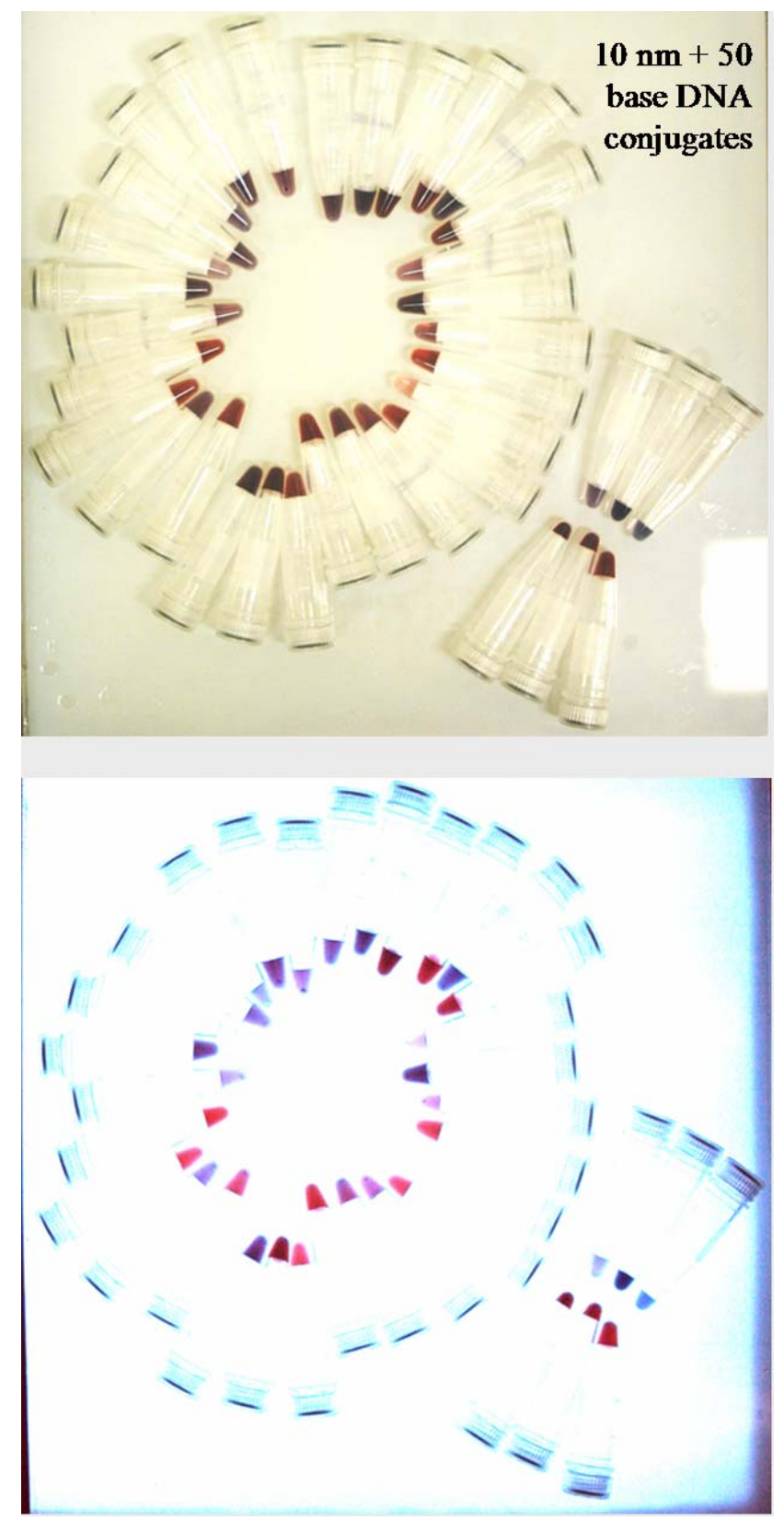

Figure 3.9: $10 \mathrm{~nm}$ gold with a single piece of 50 base (Sequence "B") DNA attached. ${ }^{3}$ The top view is an overhead-lit view, and the bottom is a transilluminated view of the same samples. Purplish or blue samples have aggregated, and are unusable for experiments. There was no difference in the preparation procedure for any of the samples, and the samples were all prepared at the same time. 
We had hoped, upon realizing the labor-intensive procedure involved in producing sufficient quantities of samples to perform hybridization reactions, to find a more elegant and productive manner of preparing a preparative amount of gold conjugate sample. Relying on the parallels between nanoparticles and proteins, we attempted to use a preparative procedure normally used to produce large quantities of purified proteins. This procedure uses the preparative tube gel apparatus photographed in Figures 2.14 and 2.15. Figure 3.10 shows a diagram of the setup. The agarose gel is poured between a ceramic cooling core and a plastic outer shell. The tube had a diameter of $3.7 \mathrm{~cm}$. For the picture in Figure 2.14, the gel was a $10 \mathrm{~cm}$ high $2 \%$ agarose gel with a $1 \mathrm{~cm} 0.25 \%$ agarose "stacking" gel on top. The stacking gel helps the sample load evenly, and prevents the top of the high-percentage gel, underneath, from puckering. In spite of the bands evident in the gel picture in Figure 2.14, the we failed to retain the separation during the collection process. ${ }^{7}$ Unfortunately, it seemed to take too long to extract the gold particles completely from the bottom the gel, where the elution frit and dialysis membrane shown in Figure 3.10 stop the gold and pull it toward the fraction collector. Fractions collected at any usable dilution (hence, still having a reddish tinge) contained mixtures of several conjugate bands. As a result, all of the experiments described here required the traditional horizontal agarose gels for the (tedious) preparatory work.

Figure 3.11 shows an overview and summary of the procedure we used to make gold trimers. First we synthesized (or bought) and purified the DNA trimers and the thiolated DNA for conjugation to the gold. Then we condensed the gold colloid to a concentration

\footnotetext{
${ }^{7}$ The sample in the picture is $10 \mathrm{~nm}$ gold with a $5^{\prime}$ thiolated 100 base DNA strand (Sequence "C") added. Sequence C is: $5^{\prime}$-HS-GCA GTA ACG CTA TGT GAC CGA GAA GGA TTC GCA TTT GTA GTC TTG AGC CCG CAC GAA ACC TGG ACA CCC CTA AGC AAC TCC GTA TCA GAT GGG AAC AGC A-3’.
} 


\section{Prep Cell Components}

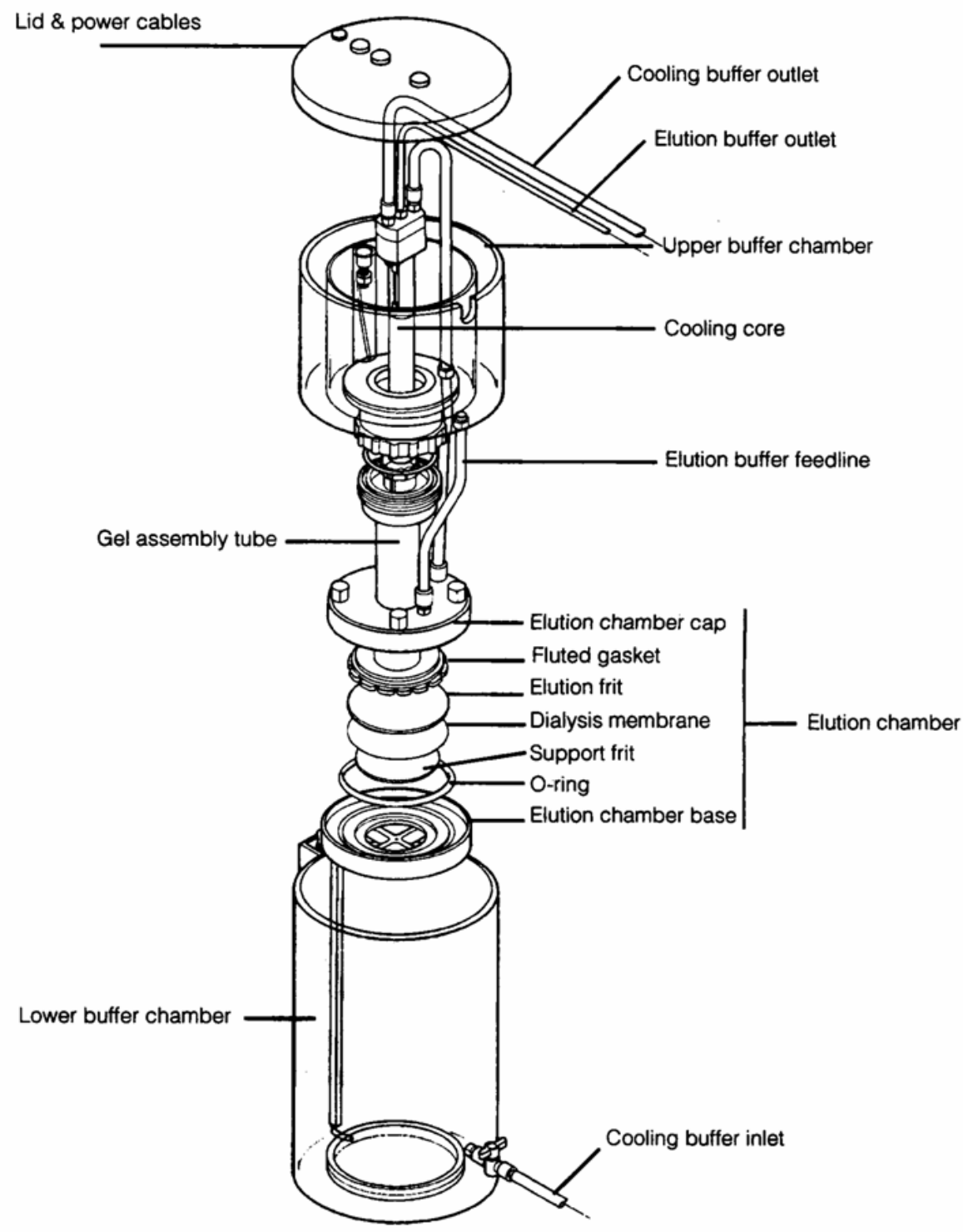

Figure 3.10: Diagram of gel separation portion of the preparative tube gel apparatus.[14, p.4] 


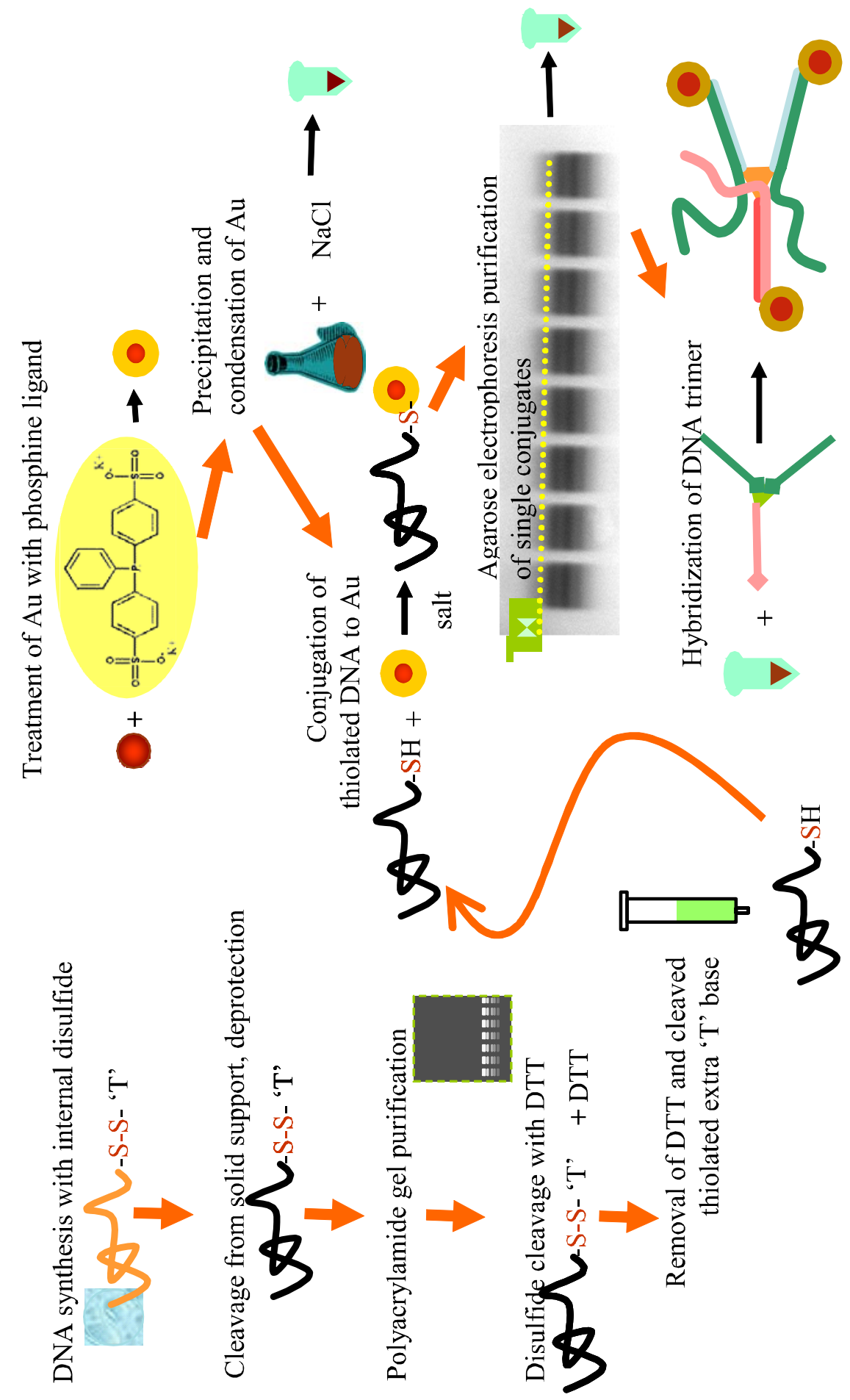

Figure 3.11: Diagram showing the procedure for making gold trimers starting with gold, thiolated DNA, and DNA trimers. 
of $1-2 \mu \mathrm{M}$ for $10 \mathrm{~nm}$ gold, or $7 \mu \mathrm{M}$ for $5 \mathrm{~nm}$ gold. We added the thiolated DNA to the condensed gold in a preparative conjugation gel, and isolated the band corresponding to the single conjugate. Then we condensed the isolate, and added it in an excess ratio to the DNA trimer in the presence of salt $(50 \mathrm{mM})$ at room temperature for a period of overnight to 5 days to allow the trimers to hybridize.

\subsection{DNA Trimer Synthesis}

We synthesized the DNA trimer ourselves by the doubling synthon method described in Section 2.3.4. Because the DNA trimer has 54 bases, and since HPLC is not the most effective method for purifying DNA of that length, we purified the DNA on PAGE gels, as detailed in Section 2.3.1. A representative denaturing PAGE gel from a 54 base branched trimer synthesis is shown in Figure 3.12. ${ }^{8}$ After purifying the trimer, we compared it to possible contamination sequences comprised of the same sequence, but unbranched. The first comparison, an 18 base strand, was the same sequence as the DNA up to the branch point. The second comparison was a 36 base strand with the same sequence up to the branch point, and then the same sequence after the branch point, only with no branch inserted, so that it was regular single strand. The third comparison was a 54 mer, with exact same sequence, and with the same approximate molecular weight as the branched DNA, but unbranched. ${ }^{9}$ These components were tested by MALDI (Section 2.3.3 discussed this

\footnotetext{
${ }^{8}$ The sequence of this DNA (Sequence "D") is: $2 \mathrm{X}\left(5^{\prime}\right.$-GAT GGT CCT TGG TGG CGG)-branch-GTC ACA TAG CGT TAC TGC-3'.

${ }^{9}$ The strands of these species were: the 18 mer (Sequence E), $5^{\prime}$-GTC ACA TAG CGT TAC TGC-3'; the 36 mer (Sequence F), 5'-GAT GGT CCT TGG TGG CGG GTC ACA TAG CGT TAC TGC-3'; the 54 mer (Sequence G) 5'-GAT GGT CCT TGG TGG CGG GAT GGT CCT TGG TGG CGG GTC ACA TAG CGT TAC TGC-3'; the branched 54 mer, Sequence "D" 2X(5'-GAT GGT CCT TGG TGG CGG)-branch-GTC ACA TAG CGT TAC TGC-3'.
} 


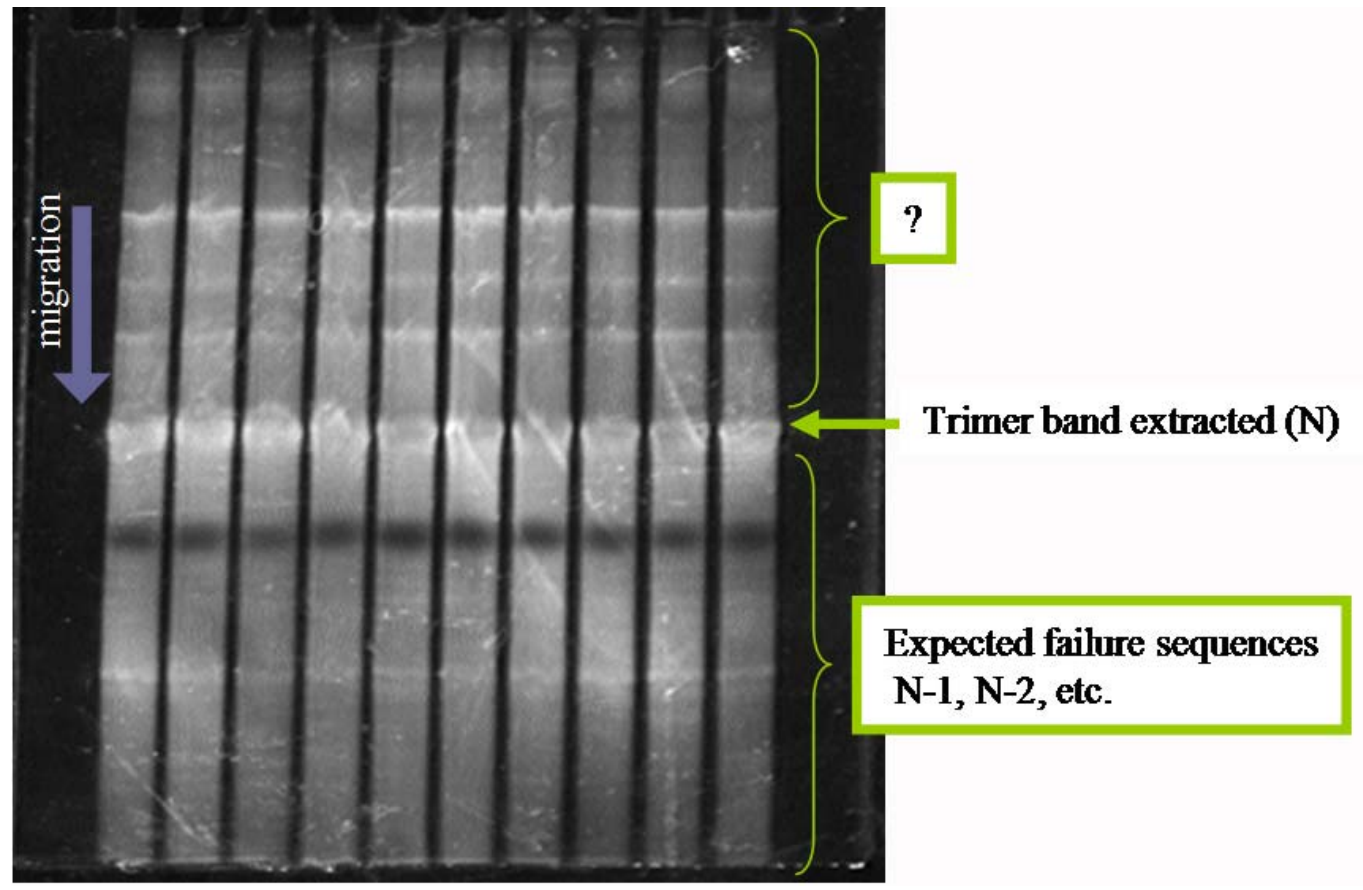

Figure 3.12: Representative branched DNA trimer preparative gel. The gel is $0.75 \mathrm{~mm}$ $10 \%$ denaturing PAGE, loaded at $20 \mu \mathrm{g}$ per lane, and run at $500 \mathrm{~V}$ and $50^{\circ} \mathrm{C}$ until the loading dye (not shown) migrated a convenient distance to ensure separation of impurities. The central whitest band is the fluorescing, GelStar-stained branched structure we desired. The remainder of the fluorescence in each lane is from impurities, such as N-1, N-2, etc. strands, and from longer unintended synthesis products. We extracted only the desired full-length band from the gel. 


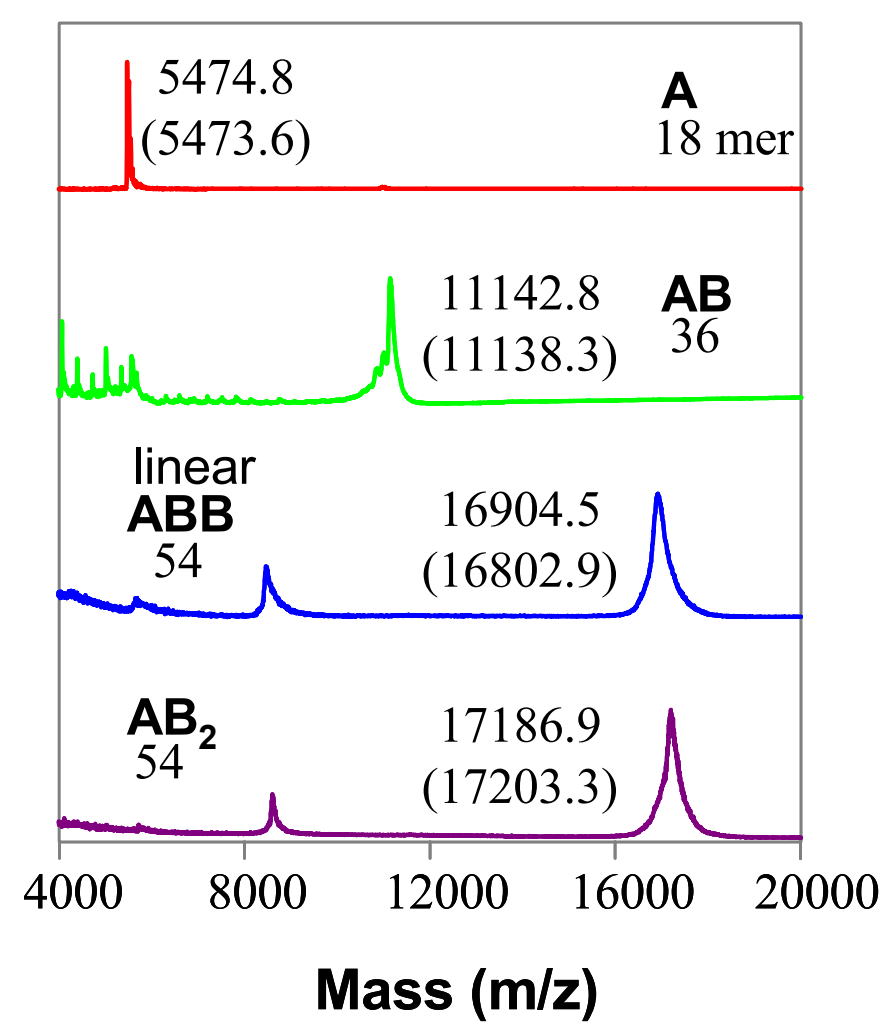

Figure 3.13: Comparison of three linear DNA components, same-sequence, by MALDI to the purified, branched DNA trimer.

technique) to check that they had the intended molecular weight, and also reasonable purity. The result of this check is shown in Figure 3.13. This figure shows that the branched trimer is quite pure. The trace for both the linear and branched 54 base strands shows one additional peak that corresponds to a doubly-ionized species. The linear dimer (36 base strand) shows some low-molecular-weight impurities, but since we had already purified it twice (by HPLC), and since it was not used for the actual gold experiment, we did not purify it yet again. We compared these same four strands in the denaturing PAGE gel in Figure 3.14 to check purity and compare migration properties (primarily to confirm that the DNA trimer was, indeed, branched). The gel in Figure 3.14 shows the branched 54 
$\begin{array}{lllll}1 & 2 & 3 & 4 & 5\end{array}$

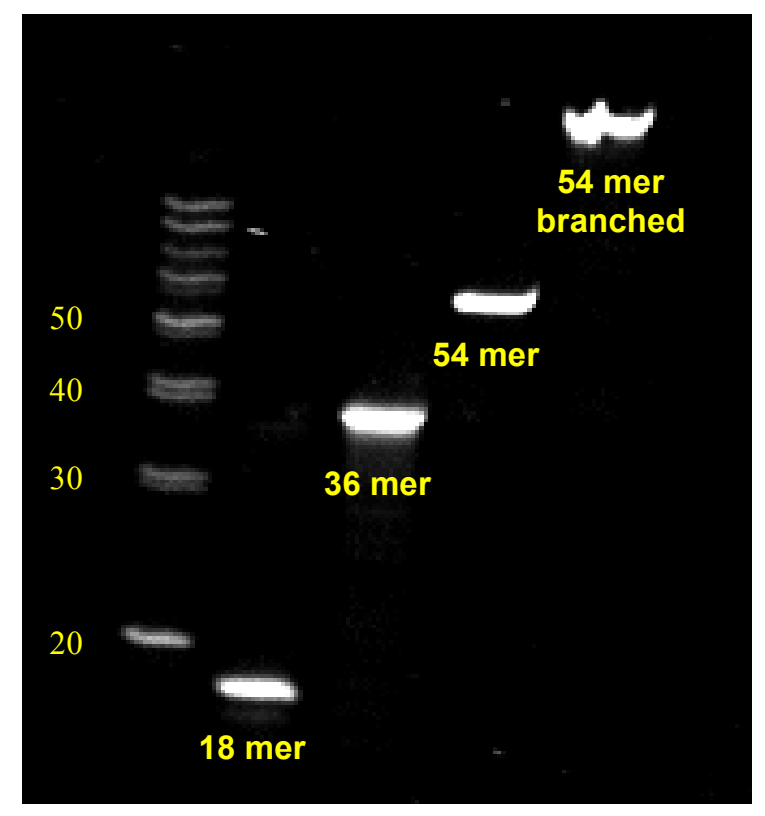

Figure 3.14: 20\% denaturing PAGE gel showing the same trimer and standards as used in the MALDI in Figure 3.13. The branched 54 mer in Lane 5 plainly migrates much more slowly than the linear one in Lane 4 . The first lane is loaded with a 10 base ladder standard. 
base DNA plainly migrating at a slower rate than the linear DNA with the same sequence, which is what we would expect in the case of a branched piece of DNA.

In order to produce the structure in Figure 3.2, we needed to add the DNA trimer to a mixture of two types of gold/DNA conjugates. One type of conjugate needed $5 \mathrm{~nm}$ gold particles with a 5'-thiolated 50 base strand, and the other needed $5 \mathrm{~nm}$ gold particles with a $3^{\prime}$-thiolated 50 base strand. ${ }^{10}$ These strands were longer than the arms of the branched trimer (because of the difficulty of synthesizing trimers with longer arms). Before performing the experiment including the gold particles, we first verified that the DNA trimer would hybridize properly to these longer strands using a native PAGE gel, shown in Figure 3.15 (shown earlier, also, in Figure 2.11). ${ }^{11}$ From this gel, we concluded that the DNA trimer structure behaved as we expected, and we then proceeded with the experiment using gold nanoparticles. We reacted 5:1 and 3:1 stoichiometric ratios of conjugate to trimer to give the results in Figure 3.16. When the third, uppermost band was isolated and placed under TEM, we saw images such as the one in Figure 3.17. A reasonable percentage of the gold particles seemed to participate in trimer-like structures, and we verified this fact using a statistical analysis program designed by Christine Micheel.[31] For her analysis, she compared the percentage of gold participating in trimer structures with that of a control sample deposited in the same manner and at a similar concentration. Those results are shown in Figure 3.18. The statistics show a majority of the gold particles in the reaction sample participating in a trimer structure.

\footnotetext{
${ }^{10}$ These DNA strands were Sequence "H" 5'-HS-GCA GTA ACG CTA TGT GAC CGG GTT CAA TTC GCA TTT GTA GTC TTG AGC CC-3' and Sequence "A."8

${ }^{11}$ The sequences for this gel were "I": 5'-TAC CCA CAC TAT CAT CAC CGC TGC TAC CAG ATC CGC CAC CAA GGA CCA TC-SH-3' and "H," the 50 base strands (unthiolated, here), and "D," the DNA trimer. ${ }^{3810}$
} 


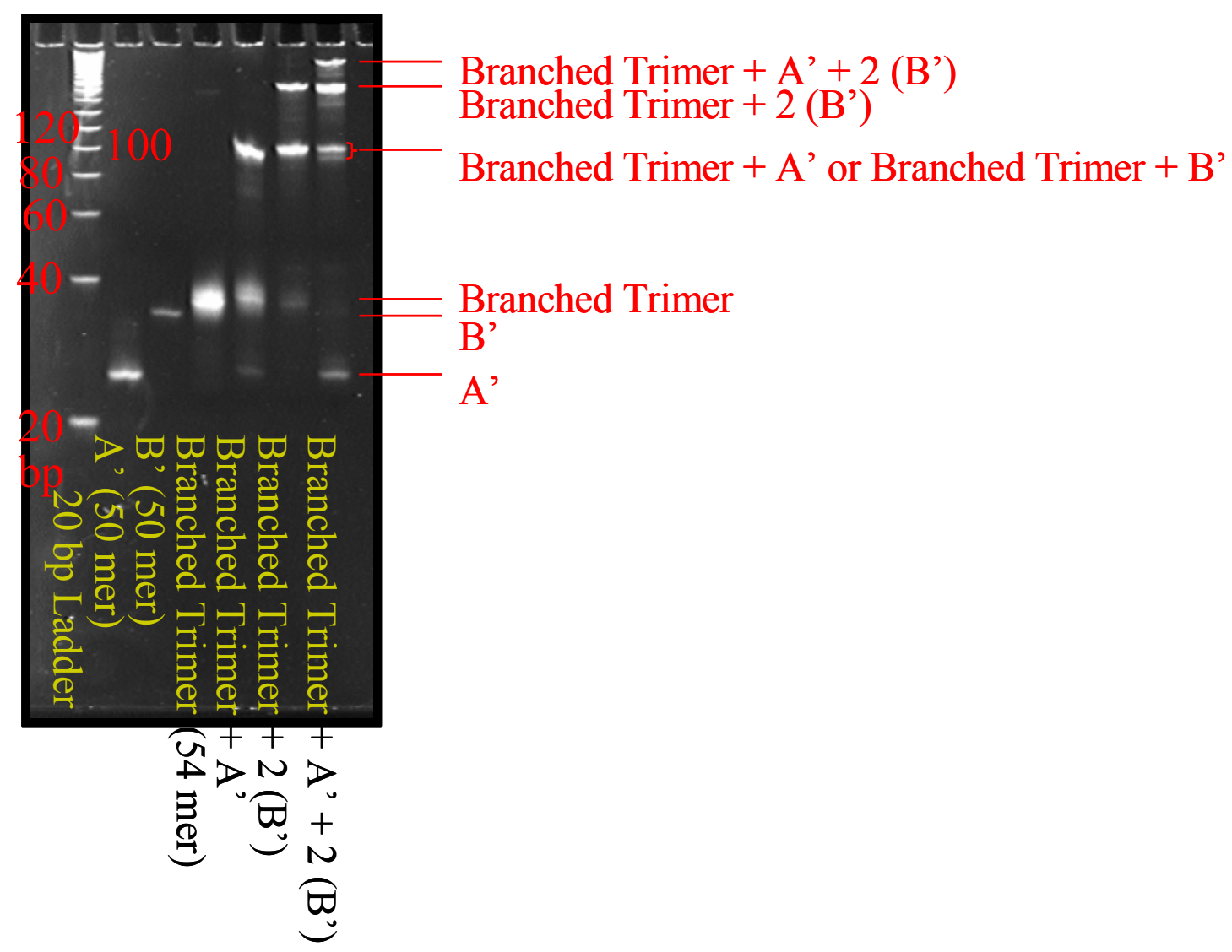

Figure 3.15: 12\% native PAGE gel showing the successful hybridization of all arms of the DNA trimer we synthesized. The leftmost lane contains a 20 bp ladder standard. The next two lanes contain the unthiolated 50 base strands that complement the three trimer arms. (Each trimer requires one "A'" strand, and two "B'" strands for complete hybridization.) The fourth lane contains the branched trimer species. The fifth lane contains the trimer plus one equivalent of A', and the sixth lane contains the trimer plus two equivalents of B'. The last lane contains the trimer plus one equivalent of A' and two equivalents of B'. The uppermost retarded band in this lane is not present in any other lane, and it contains the completely hybridized trimer. The gel was run at $4{ }^{\circ} \mathrm{C}$ for about $5 \mathrm{hrs}$. at $160 \mathrm{~V}$ in $1 \mathrm{X}$ TBE. (Also shown as an example of a generic native gel in Figure 2.11.) 


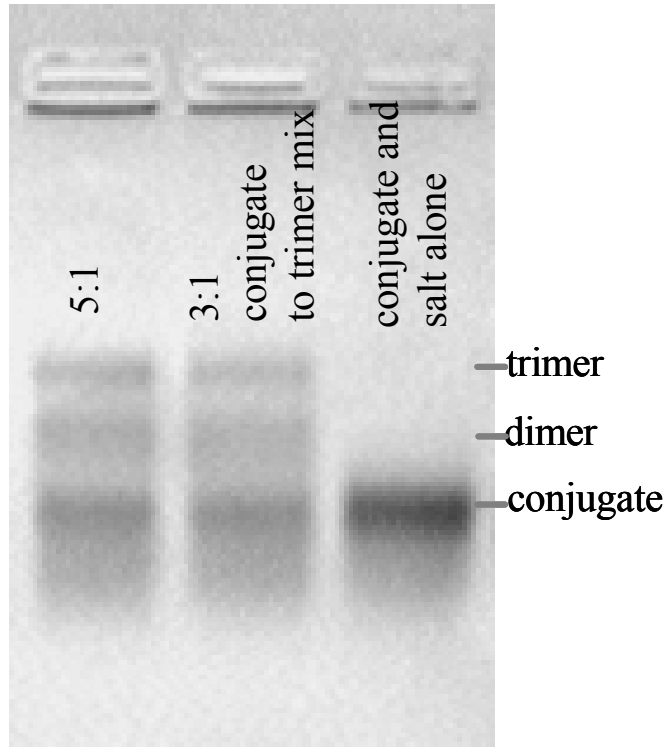

Figure 3.16: 5:1 and 3:1 conjugate to trimer ratio reactions annealed at room temperature for five days. The reactions had a 2:1 mixture of $5 \mathrm{~nm}$ gold conjugates with Sequence A to those with Sequence H, and they were $0.84 \mu \mathrm{M}$ in gold, overall, with $50 \mathrm{mM} \mathrm{NaCl}$. The gel was $3 \%$ agarose run in $0.5 \mathrm{X}$ TBE until the bands achieved sufficient separation. The control lane contains both conjugates and salt, but no trimer. 


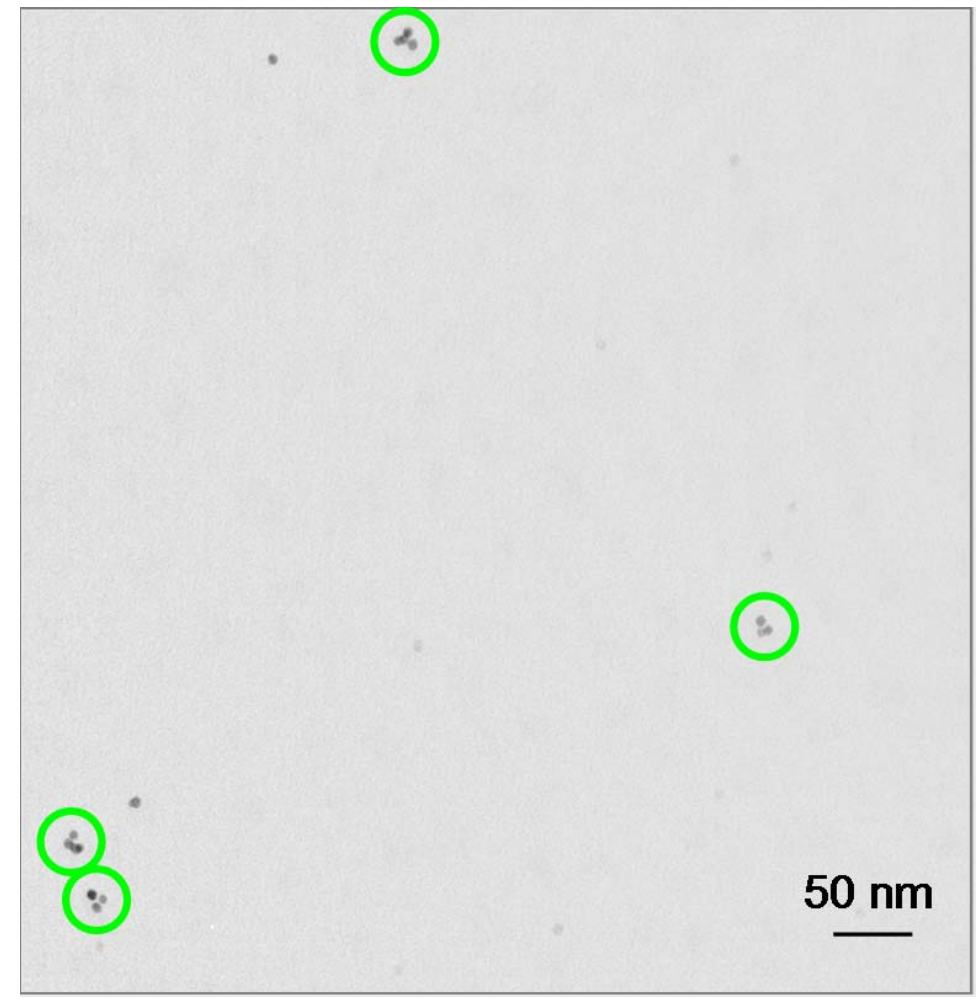

Figure 3.17: TEM image of the sample extracted from the third, uppermost bands in the gel in Figure 3.16.

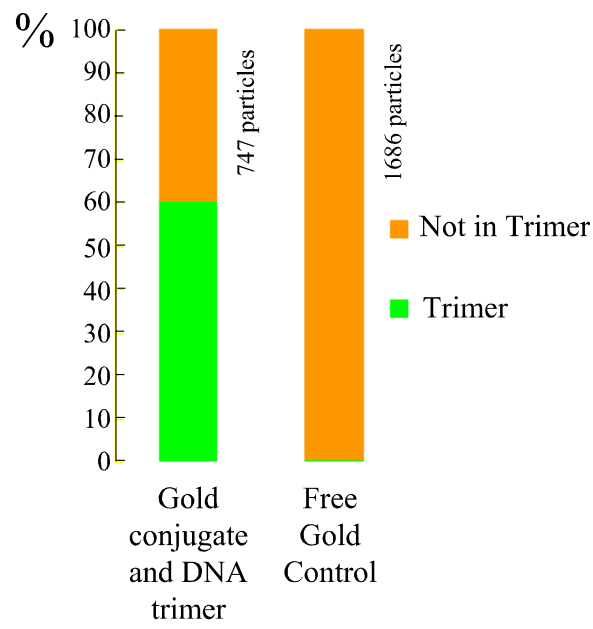

Figure 3.18: Comparison of the percentage of gold particles in trimers for the gel-extracted trimer sample, and for a control of free gold particles. 


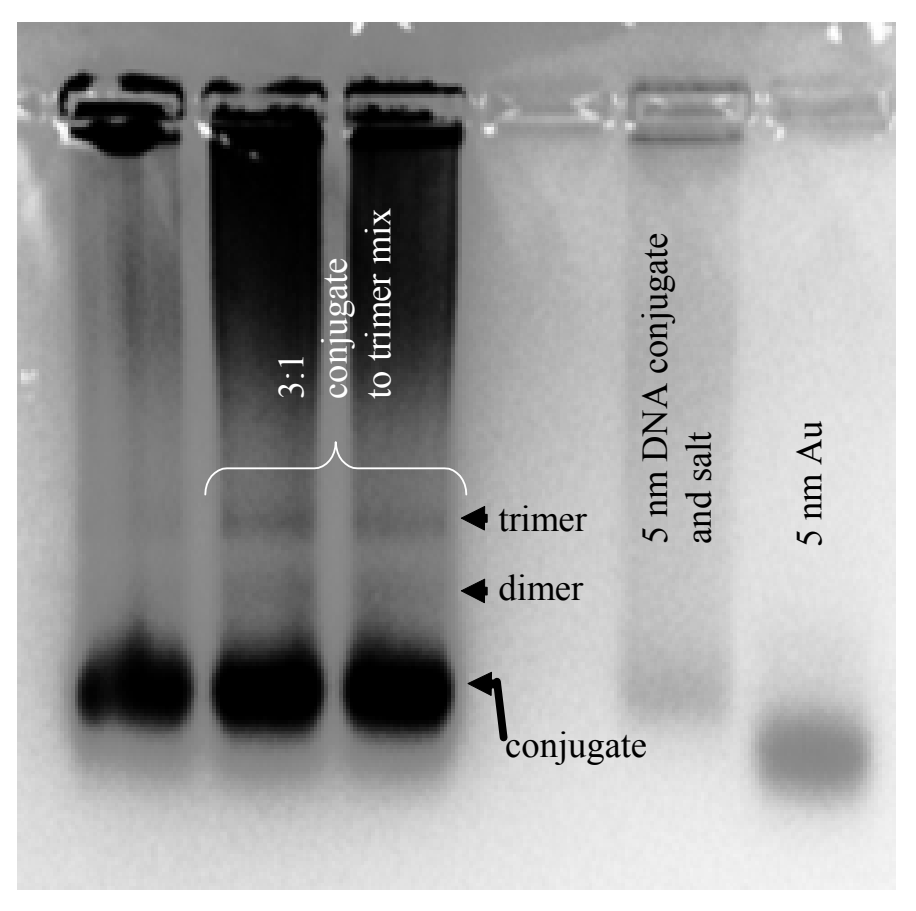

Figure 3.19: 3\% agarose gel showing the results of a repeat of the experiment in Figure 3.16. This reaction was a 3:1 reaction of the conjugates to DNA trimer (this time trimer Sequence "J" and conjugates with Sequences "A" and "H"). Three bands are once again visible in the reaction lane, although they are fainter than in Figure 3.16. Unlike the five-day reaction in Figure 3.16, this reaction was only annealed overnight.

This result alone would be great, except that further experiments did not give such conclusive results.

A repeat of this experiment gave the results shown in the gel in Figure 3.19. When we isolated topmost band in this gel, and imaged it (as shown in Figure 3.20), we did not see the trimers that we saw before in Figure 3.17. When we performed a statistical analysis of the TEM results compared to the second band in the gel, the results, shown in Figure 3.21, were not promising.

We tried a similar experiment using an asymmetric gold trimer structure having both 5 and $10 \mathrm{~nm}$ gold nanoparticles. The $10 \mathrm{~nm}$ gold samples were more difficult to prepare 


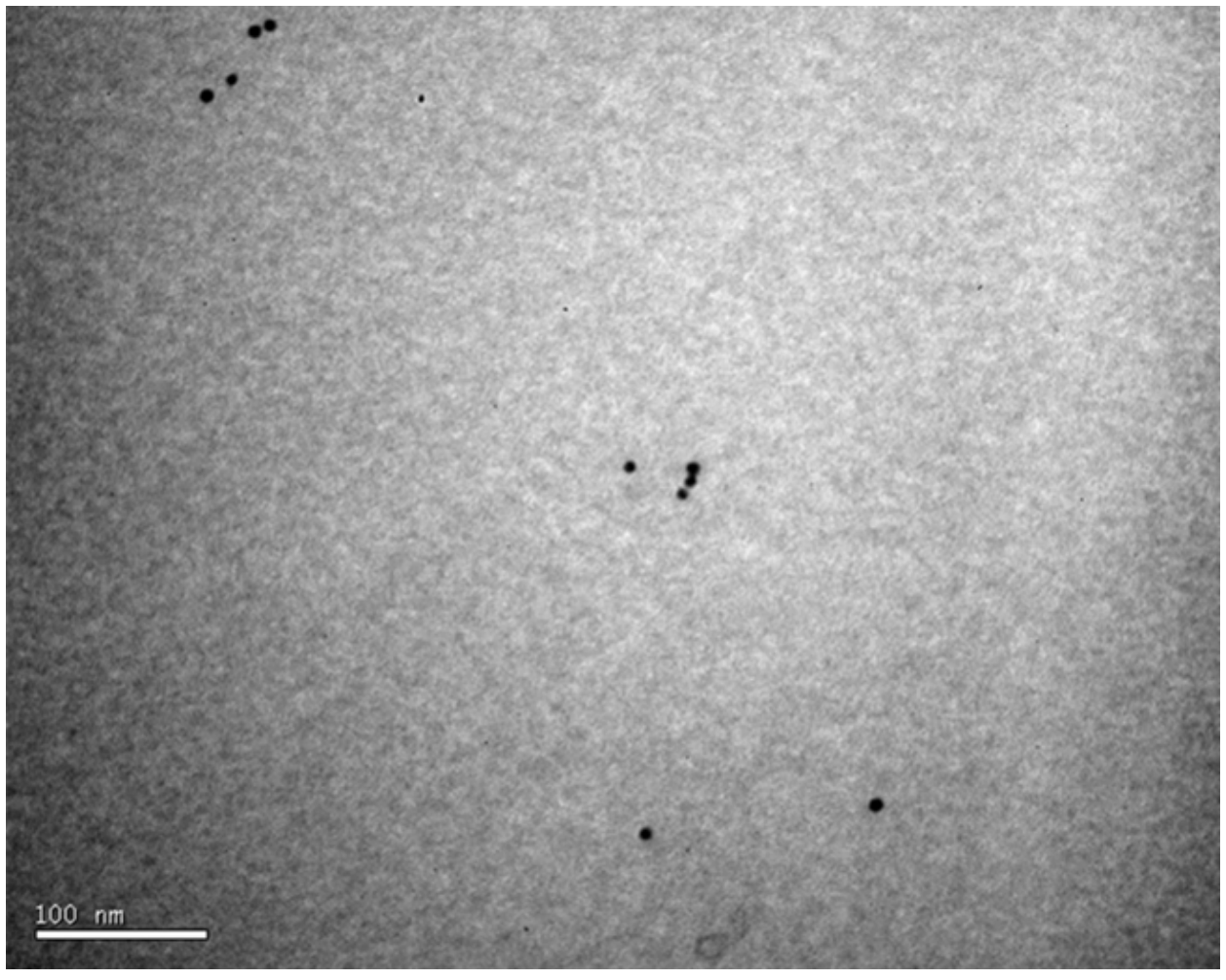

Figure 3.20: TEM image of topmost extracted band in gel shown in Figure 3.19. Trimer structures are not prevalent. 


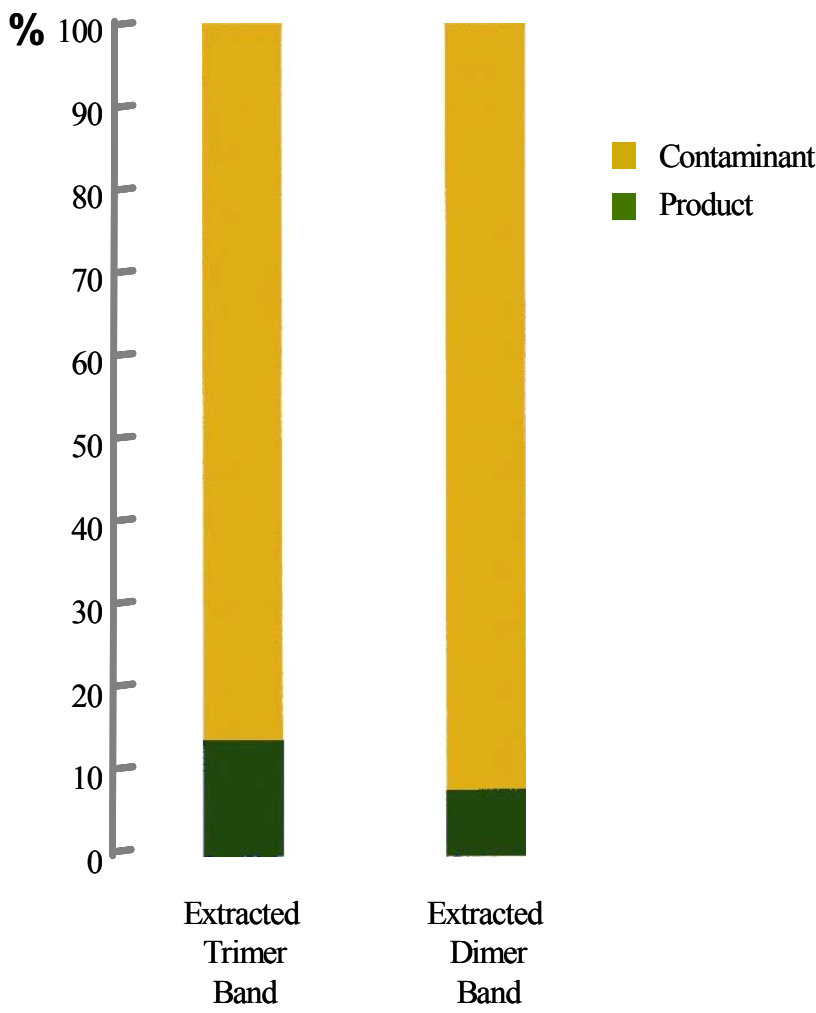

Figure 3.21: Results from a statistical analysis of the TEM data in Figure 3.20 from samples extracted from the gel in Figure 3.19. The two uppermost gel bands were analyzed. The percentage of trimers was small, and not much larger than were in the other band, not expected to contain them. 


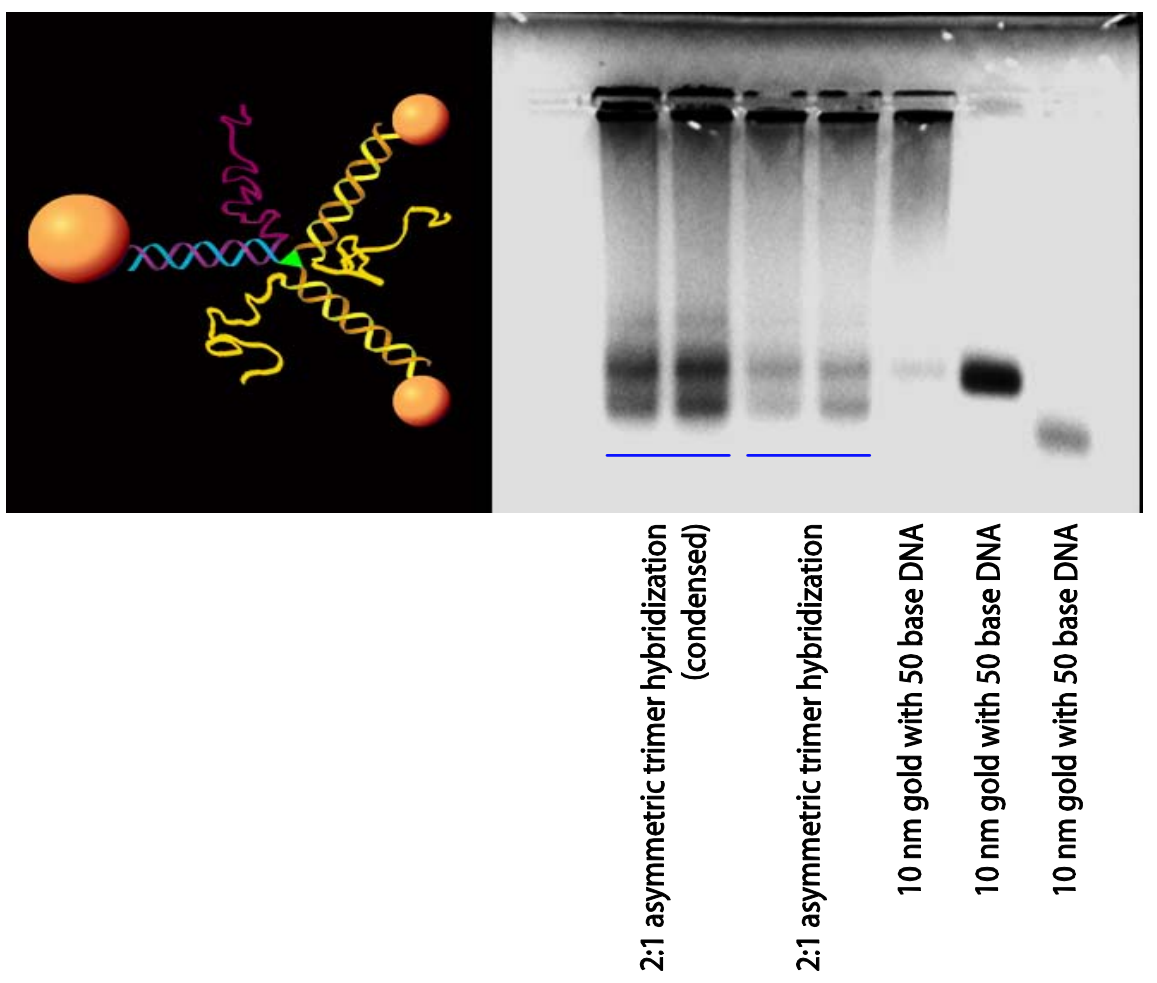

Figure 3.22: $3 \%$ agarose gel showing the results of hybridizing a DNA trimer with 5 and $10 \mathrm{~nm}$ gold nanoparticles. The $10 \mathrm{~nm}$ gold nanoparticles had a tendency to aggregate, shown by the presence of aggregates in the wells in lanes containing $10 \mathrm{~nm}$ gold. A band corresponding to a successful trimer reaction is not visible. This reaction was annealed overnight.

because they had a greater tendency to aggregate. Figure 3.22 shows a gel containing the results of a reaction with one $10 \mathrm{~nm}$ particle and two $5 \mathrm{~nm}$ particles hybridizing to each DNA trimer. ${ }^{12}$ We would expect this gel to have at least a few more bands than the gels with totally symmetric trimers, because of the presence of conjugates with a different size of gold. Actually, we could have up to seven different species in the reaction mixture, as shown in Figure 3.23. At the time, we did not expect gold conjugates with a DNA trimer attached to migrate differently (in a way we could resolve, that is) than the conjugates alone. In that

\footnotetext{
${ }^{12}$ This experiment used a trimer with Sequence "J": 2X(5'-ACT GTC CAG GTT TCG TGC)-branchGTC ACA TAG CGT TAC TGC-3', $5 \mathrm{~nm}$ conjugates with Sequence "A," and $10 \mathrm{~nm}$ conjugates with Sequence "H." 310
} 


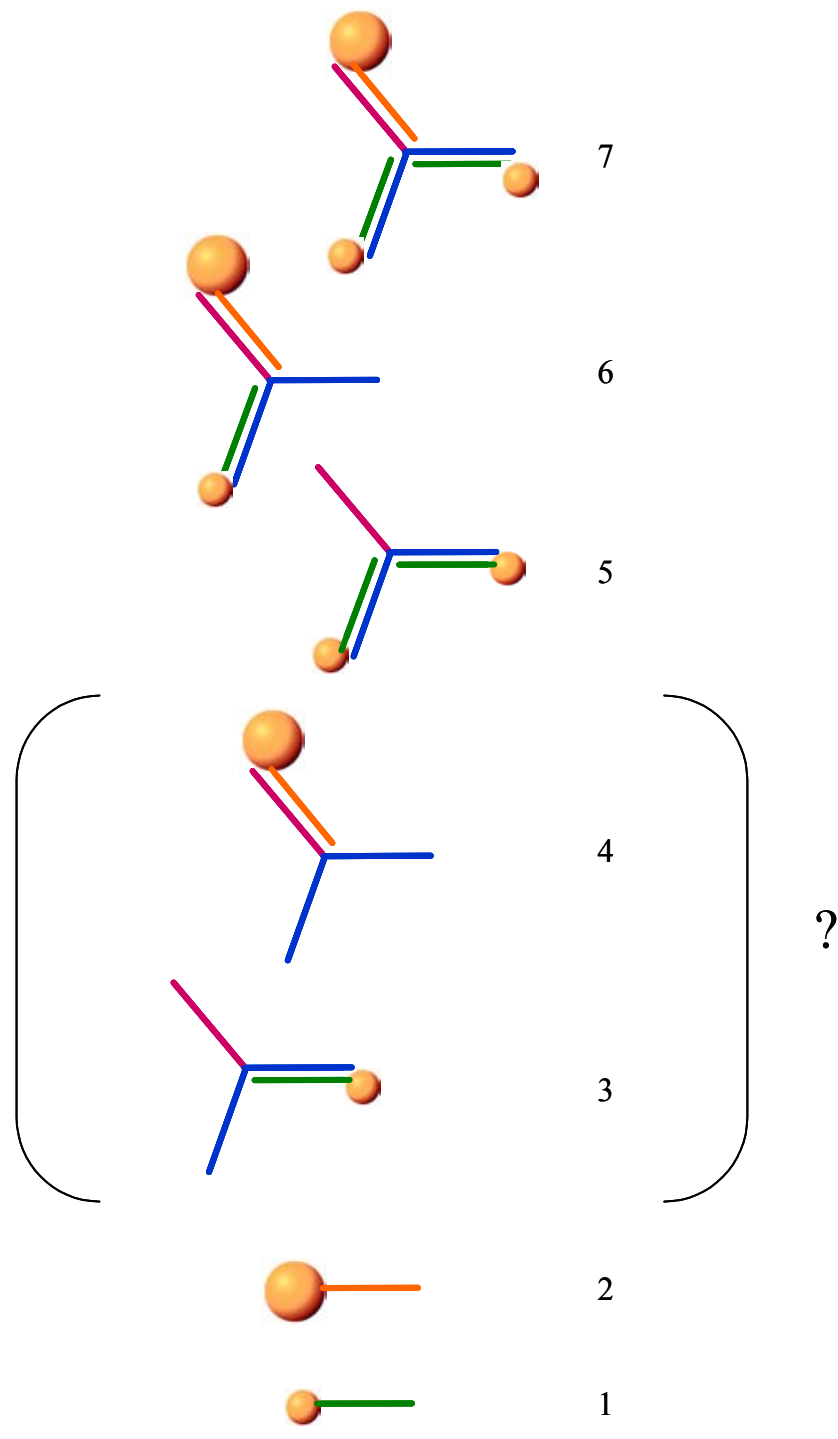

Figure 3.23: Diagram of possible species that might exist after annealing in an asymmetric trimer hybridization reaction. Number 1 labels the (most probable) fastest migrating species in a gel. Up to seven unique species may exist. At the time of this experiment it was unclear whether species 3 would migrate separately from 1, and whether 4 would migrate separately from 2. Would addition of a branched DNA strand 54 bases long perturb the 50 base gold conjugate enough to resolve a difference? We thought not, in reference to the results shown in Figure 3.16. If we had resolved that difference, we thought, there would have to be four bands showing in that gel, and there were only three. 


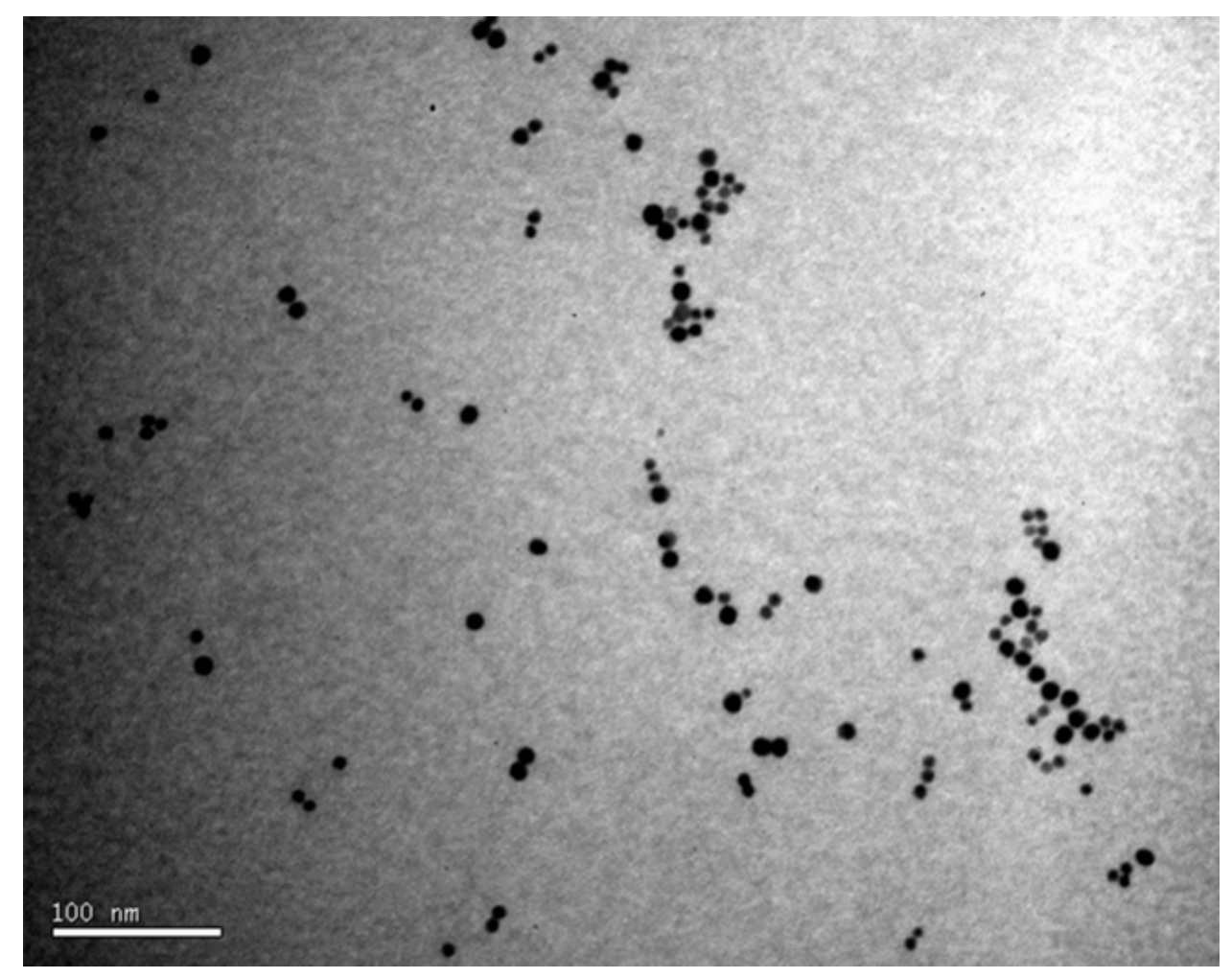

Figure 3.24: TEM of the uppermost band in the gel in Figure 3.22. There is no clear indication of any preponderance of one type of species in this picture, or in the other pictures taken at the same time.

case, we would expect to see only five bands in the gel, if they all could be resolved. These bands, if visible, would correspond to $5 \mathrm{~nm}$ conjugates, $10 \mathrm{~nm}$ conjugates, $5+10,5+5$, and the complete trimer. In the gel in Figure 3.22, however, we see only three bands. It is unclear whether longer annealing would have improved these results, or if adjacent bands are just not distinct because the different species have mobilities that are too similar. TEM of the uppermost band only showed a disappointing jumble of aggregates, shown Figure 3.24. A statistical analysis of the results confirmed problems with this hybridization reaction. Figure 3.25 shows the analysis results. These results were not at all convincing, and the presence of the additional size of gold particle (the $10 \mathrm{~nm}$ conjugate) made the experiment 


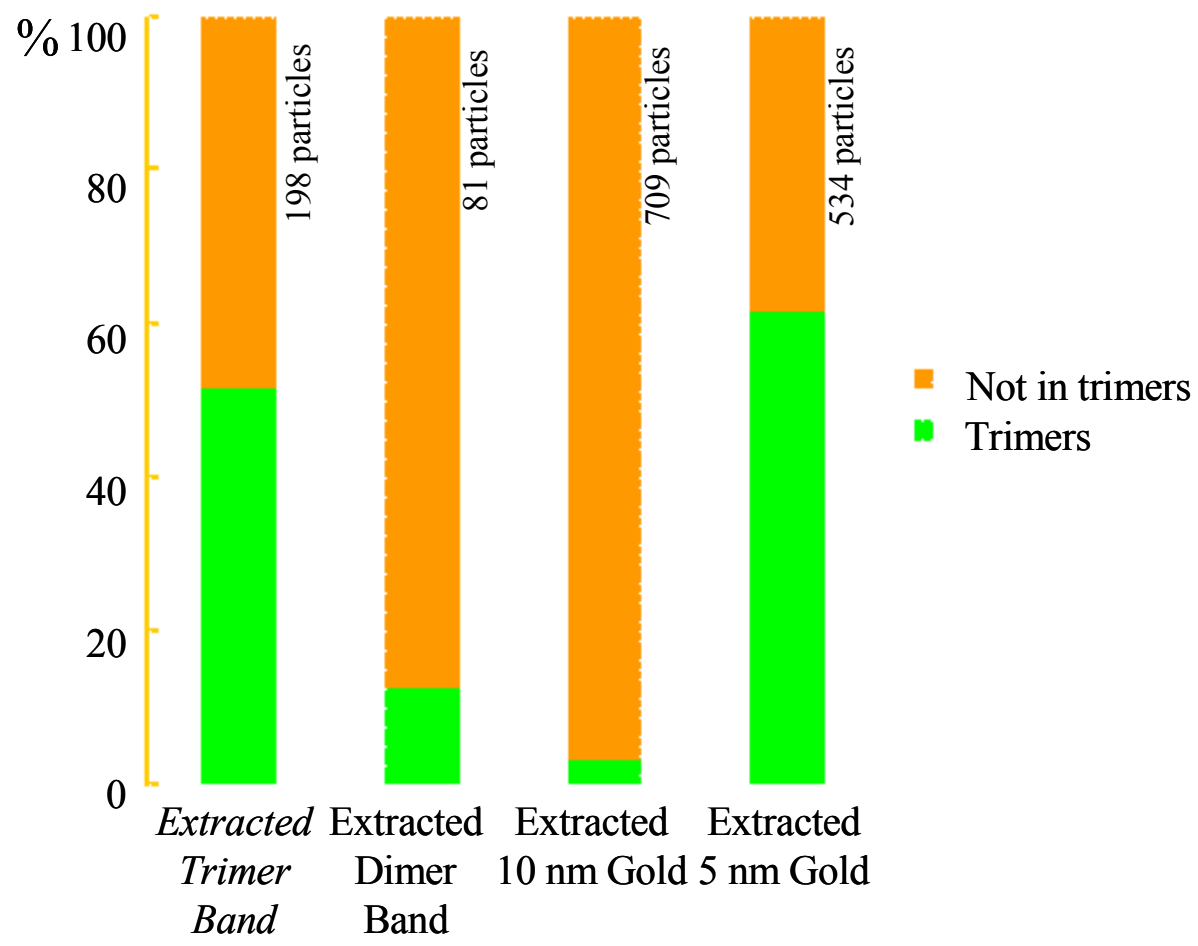

Figure 3.25: Statistical analysis of TEM images taken of the topmost band in the gel shown in Figure 3.22. The $5 \mathrm{~nm}$ extracted gold control in this experiment was deposited at too high of a concentration, giving an abnormally high result for trimers in the free gold sample. The results, especially in light of the unconvincing TEM picture in Figure 3.24, are not conclusive. 


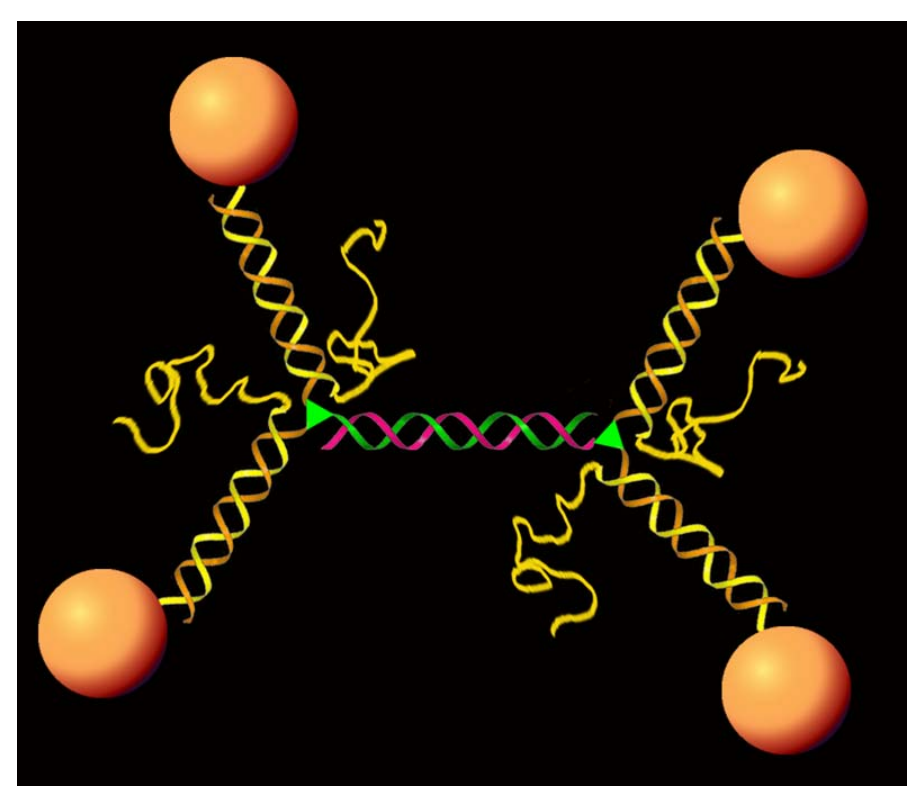

Figure 3.26: Diagram of the target structure of a totally symmetric tetramer comprised of two DNA trimers and a single type of $5 \mathrm{~nm}$ gold conjugates.

more challenging. There were greater problems with sample aggregation, and a larger number of structures that could result from the hybridization reaction. We chose to focus on experiments only incorporating $5 \mathrm{~nm}$ gold for this reason.

We also performed experiments seeking the "double trimer" or "tetramer" structure in Figure 3.26. We expect this structure to produce up to seven distinct bands in the gel, as shown in Figure 3.27. We performed at least three reactions of this type that did not result in complete aggregation. Figure 3.28 shows these three gels. ${ }^{13}$ From these results, it seems it would be best to have the maximum ratio of gold to DNA trimer possible, constrained by the loading limitations of the wells, and also the limitations of condensing the gold conjugates. We desired the maximum ratio for the hybridization reaction, possible.

\footnotetext{
${ }^{13}$ The sequences in these three gels were trimers Sequence "K": 2X(5'-ACT GTC CAG GTT TCG TGC)branch-GCA GTA ACG CTA TGT GAC-3" and Sequence "J." We used $5 \mathrm{~nm}$ conjugates with Sequence "A." 312
} 

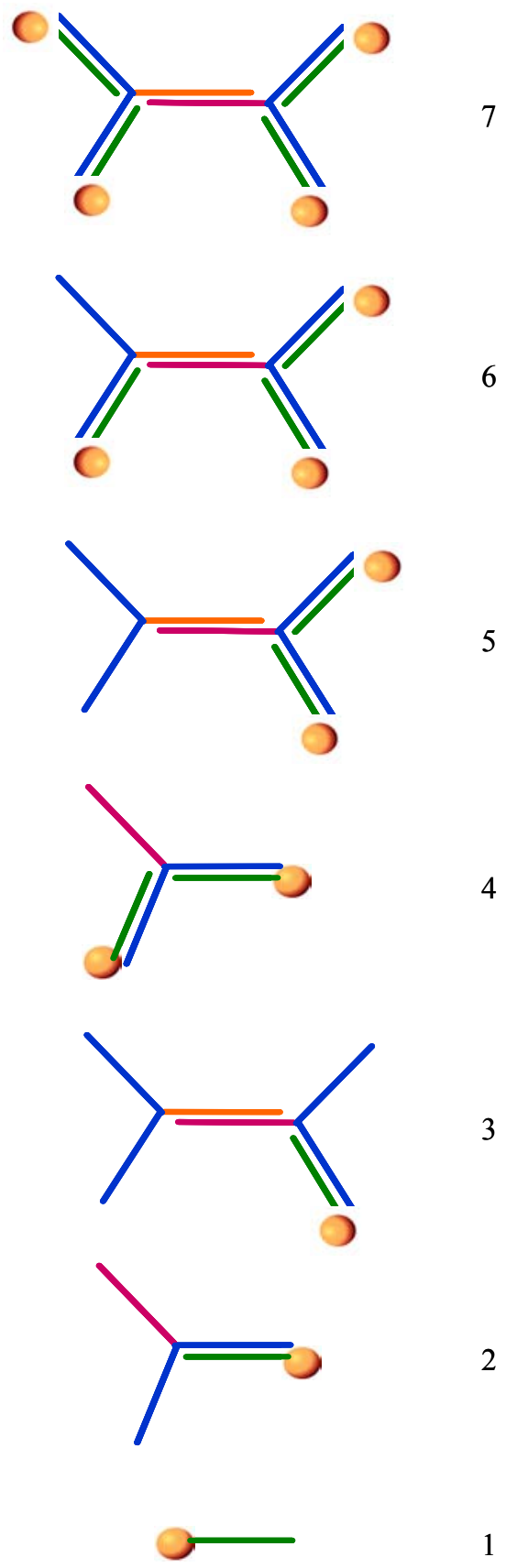

Figure 3.27: Diagram showing the seven conceptually unique species that could exist, postreaction, in a symmetric tetramer hybridization mixture. Number one labels the fastest band in the mixture. 


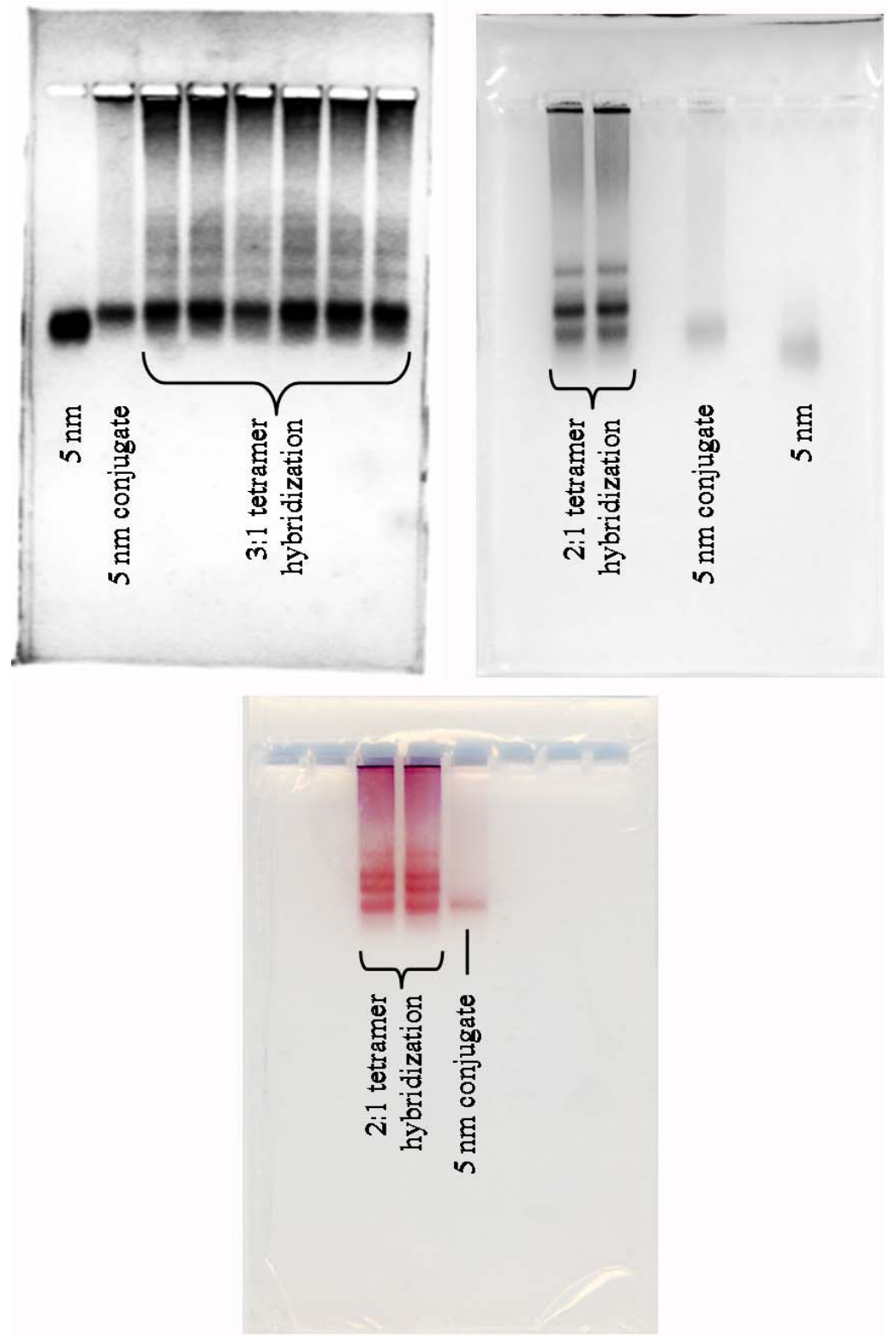

Figure 3.28: Three 3\% agarose gels showing the migration of three different reaction mixtures for a totally symmetric tetramer with $5 \mathrm{~nm}$ gold. The gel on the top-left has the most bands, with at least five, and possibly six visible. The gel on the top-right has fewer bands. The hybridization ratio of conjugates to trimer was lower in this case, so not enough active conjugates may have been present to drive the reaction to completion. (We expect some portion of the gold conjugates to be inactive, because the DNA may be non-covalently bound in some way to the gold surface.) The gel on the bottom (imaged in color) shows four bands in the reaction mixture. 
However, it was hard to condense the gold conjugate sample sufficiently to get a ratio of more than 2 or 3 without precipitating the sample. If the sample was condensed less, it was possible to add still less DNA trimer to achieve a high conjugate to trimer ratio, but then the amount of gold present in the uppermost bands would be too little to detect by eye, because only a small volume can be loaded into even a thick gel, and the amount we can detect is limited by the extinction coefficient of the gold (See Table 2.1). Perhaps a silver-enhanced gel would help overcome these detection limitations, or the use of radioactive labeling. Actually, the use of radioactive labeling probably would help our results in a lot of these (and related) experiments, but that technique had a high labor cost for establishing the necessary facilities and safety precautions, so we avoided it.

For all of the symmetric tetramer reactions, the TEM results were inconclusive. Figure 3.29 shows an example of a TEM picture taken from the uppermost band in the top-right gel in Figure 3.28. Christine Micheel performed a statistical analysis on all of the bands for this gel, and the results are shown in Figure 3.30. Most of the trouble in the TEM imaging process came from the difficulty of extracting an intact and un-aggregated sample from the gel. Once the samples entered the gel, there was no longer a surrounding bath of sodium ions present, so the DNA's double helix was probably less robust under these circumstances. We could not add salt to the electrophoresis buffer without increasing the current dramatically, which would increase joule heating of the gel and melt it. The hybridized DNA was probably able to carry sufficient ions with it during electrophoresis to retain its previously hybridized form, as demonstrated by the successful formation of simpler structures that faced the same conditions, and by native DNA agarose such as that 


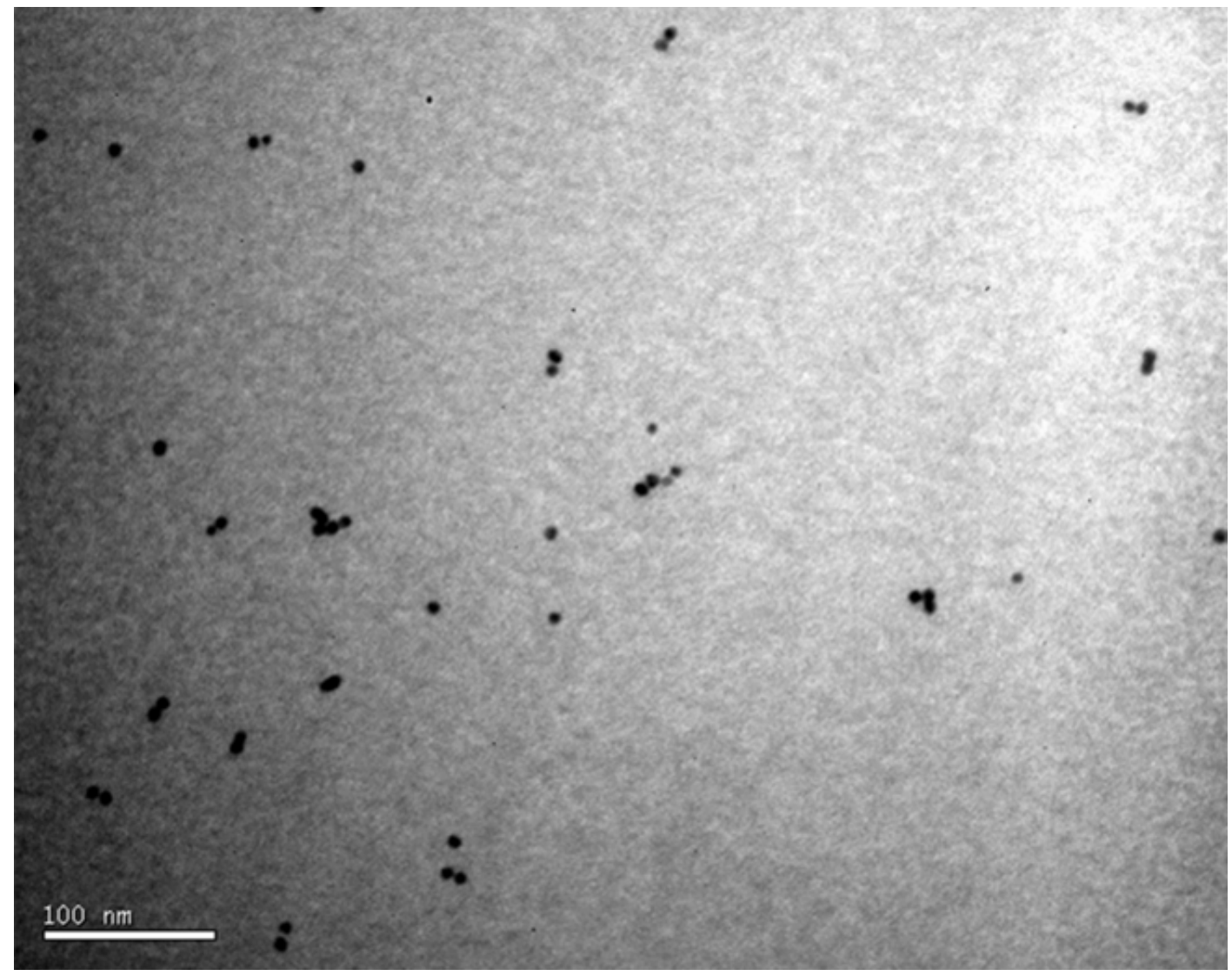

Figure 3.29: TEM looking for gold tetramer structures in the uppermost band in the topright gel in Figure 3.28. The results are visually unconvincing. 


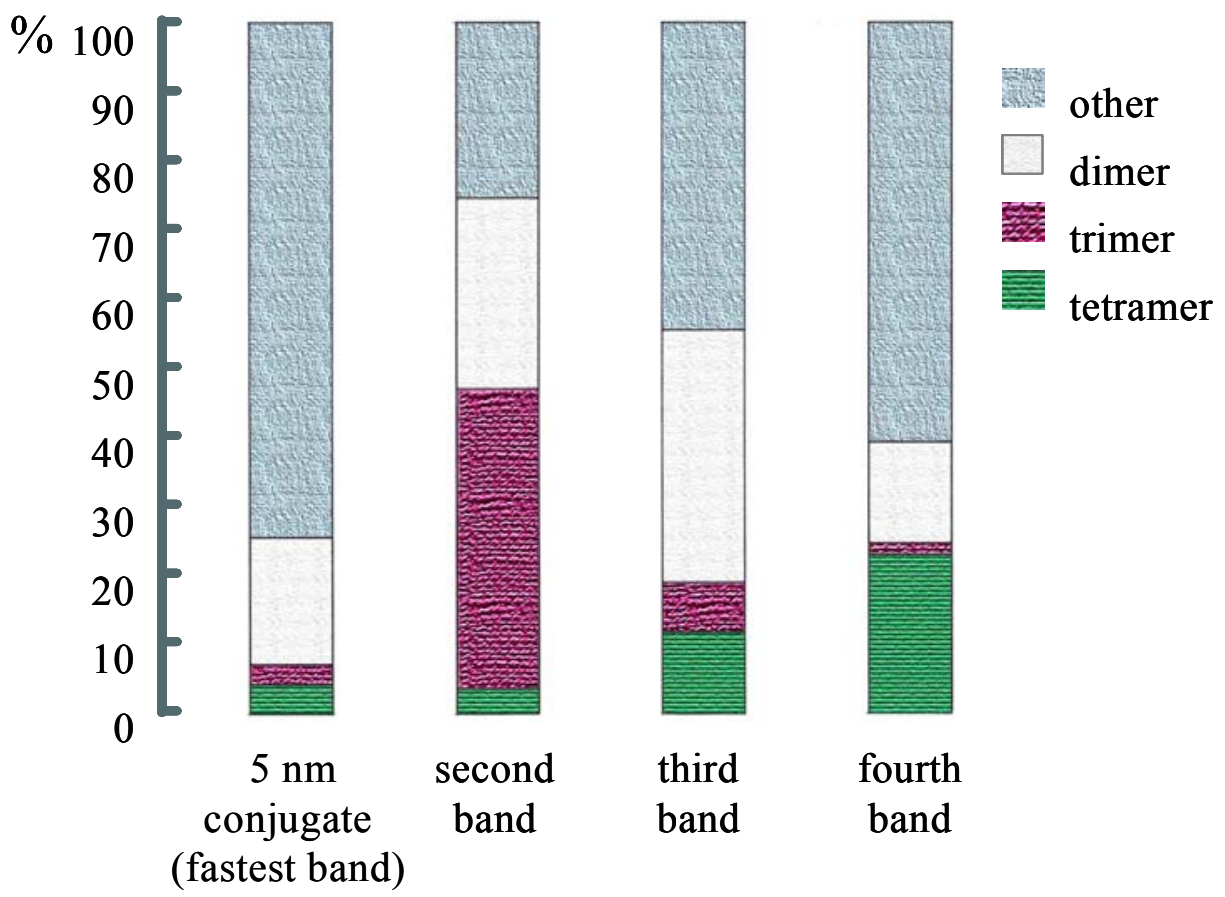

Figure 3.30: Statistics for all types of structures in each band for the top-right gel in Figure 3.28. The statistics do show an increased presence of tetramer in the fourth band, but it is not present in large quantities. "Other," which includes both single particles and larger aggregates, comprises a large portion of the structures on each TEM grid. 
shown before in Figure 2.13. Another problem we faced in extracting nanoparticles from the gel was the tendency of the sample to aggregate after extraction. We added $10 \mu \mathrm{l}$ phosphine solution $(40 \mathrm{mg} / 100 \mathrm{ml}$ water $)$ to each extracted vial to attempt to forestall this problem, but often, the sample aggregated more with each successive extraction procedure.

The last type of trimer-based structure that we attempted to create was an asymmetric tetramer. This structure incorporated two $5 \mathrm{~nm}$ gold nanoparticles and two $10 \mathrm{~nm}$ nanoparticles. The presence of two types of gold in the structure complicated the resulting bands a great deal, as we can see in the drawing of possible structures shown in Figure 3.31. When we set up this reaction, however, the number of bands visible in the gel was not so many as we expected. Figure 3.32 shows the resulting gel. ${ }^{14}$ This gel did not contain nearly enough visible bands for any of them to be a full tetramer structure-containing band, so we did not do a TEM analysis for the bands in this gel.

Gold-DNA conjugates were the main limiting reagent for these reactions. The conjugating piece of DNA was relatively short at a length of only 50 bases, and it was difficult to resolve bands for single conjugates in the gel. The experiment would have been easier if we could have used longer DNA to conjugate to the gold. However, the longest DNA we could buy that had the $3^{\prime}$ thiol we needed was only 50 bases long. Our attempts to synthesize longer 3 '-thiolated DNA ourselves failed because the DNA did not give discrete bands when conjugated to the gold. It was possible, however, to buy DNA 100 bases long with a $5^{\prime}$ thiol. We redesigned the tetramer structure to allow us to synthesize it in the opposite direction, using a special set of $5^{\prime} \rightarrow 3^{\prime}$ bases from Glen Research. ${ }^{15}$ This

\footnotetext{
${ }^{14}$ The sequences for this reaction were: $5 \mathrm{~nm}$ conjugates with Sequence "A," and $10 \mathrm{~nm}$ conjugates with Sequence "B;" Trimers with Sequences "D" and "K." 3813

${ }^{15}$ The sequences for this experiment were: Sequence "L," 5'-CAA TGT TCG TGT AAT CGG CA-
} 


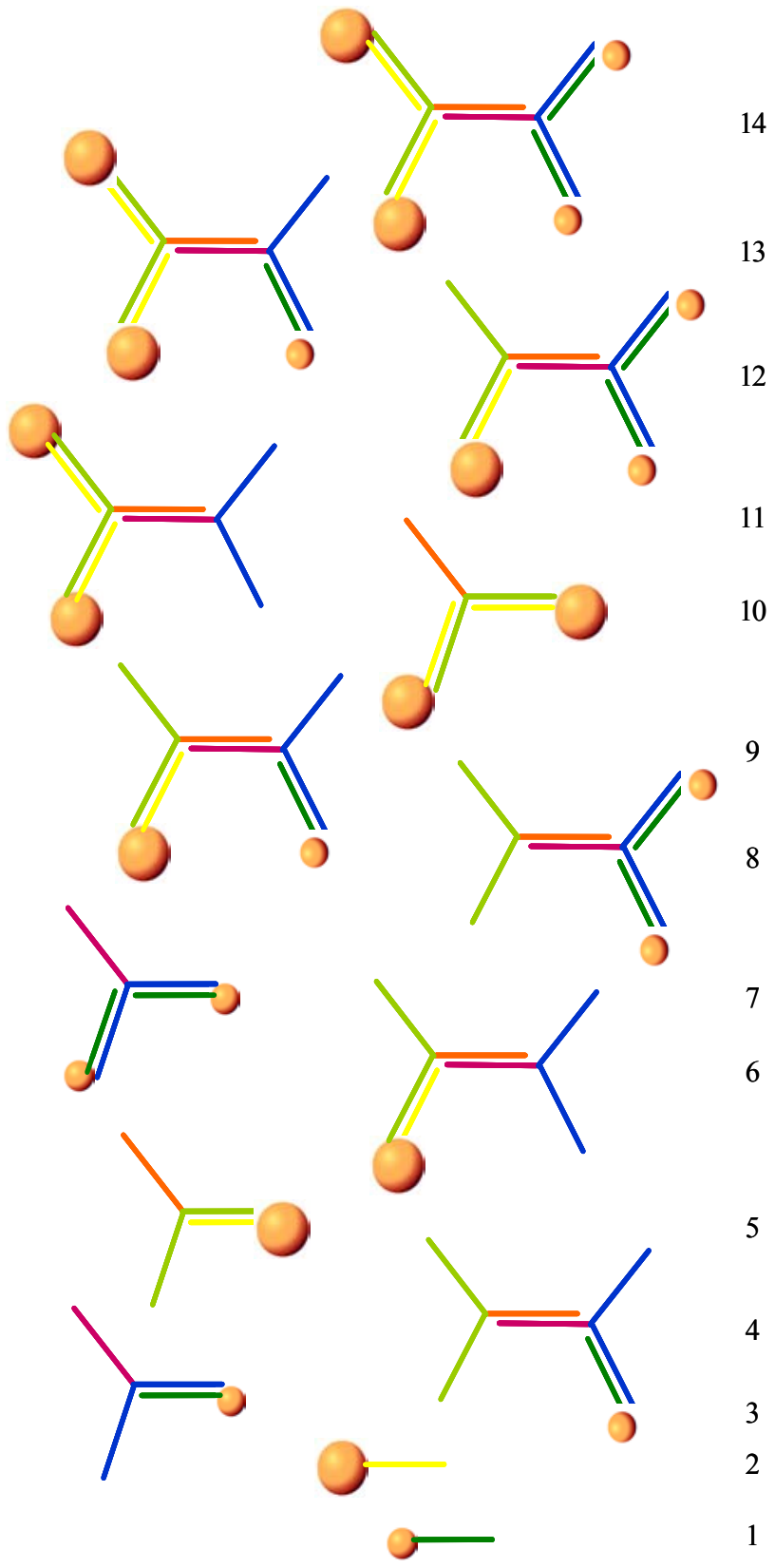

Figure 3.31: Fourteen different species that may be present in a hybridized reaction mixture for an asymmetric tetramer. As with the other similar figures, number 1 labels the fastest band. 


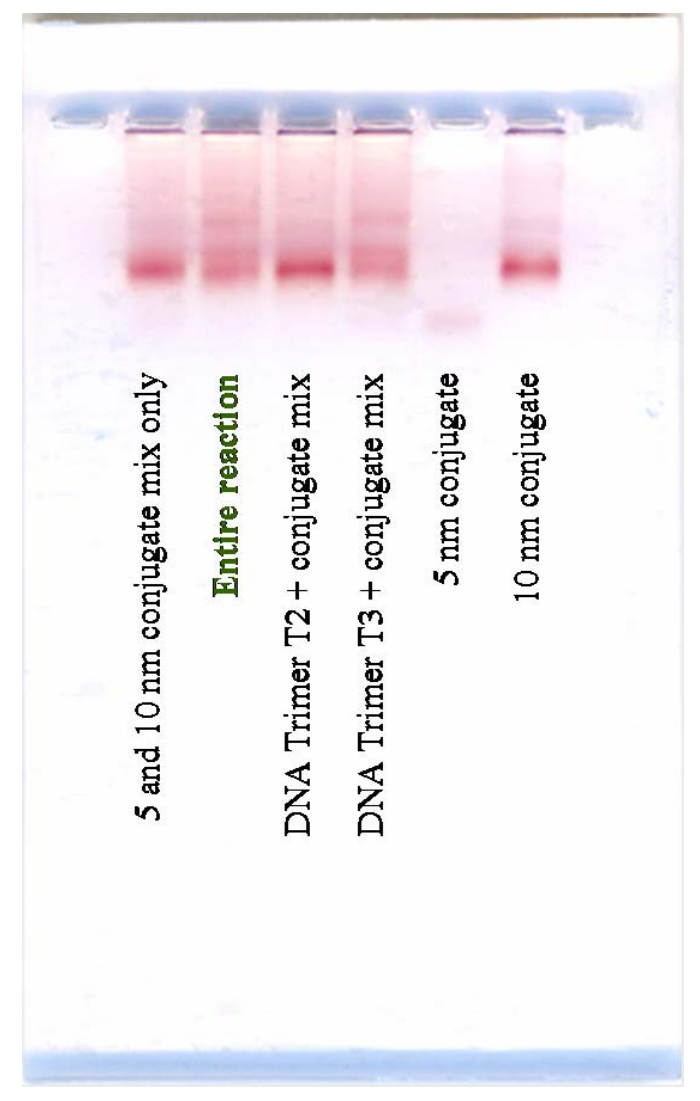

Figure 3.32: $3 \%$ agarose gel showing preliminary results for an annealing reaction of an asymmetric tetramer. The bands in this gel were quite faint; probably the reaction would have benefited from the use of more concentrated conjugates. At least three bands are visible in the reaction mixture, although up to twelve would be expected (See Figure 3.31). If there are upper bands, they are not present in great enough quantity to see. The reaction mixture containing only one of the two trimers (the fourth lane) also has the same three bands, so the uppermost band we can see would be expected to contain two gold particles hybridized to a single DNA trimer. The reaction was annealed overnight. 
modification would allow us to use 100 mer $5^{\prime}$-thiolated DNA for the four gold particleconjugates in the tetramer structure. When we tried annealing this sequence without the gold, however, the two halves of the tetramer failed to join, as seen in Figure 3.33. We are not sure why the two trimer halves failed to join in this experiment. After realizing this problem, we abandoned attempts to synthesize the DNA trimers in the reverse direction.

When we saw the results from the experiment in Figure 3.16, designed to make the totally symmetric trimer, we came to the conclusion we had made it because the gel had three distinct bands (presumably corresponding to 1, 2, and 3 golds in the structures), and because TEM of the uppermost band showed a significant number of trimers (see Figures 3.17 and 3.18). The gel results of the other repeat experiment, shown in Figure 3.34, conflicted with that conclusion, however. Three bands are distinct in each of the gels, and because each half of the tetramer can hybridize a maximum of two gold particles, the assignment of each band is fairly obvious (shown in the labeling in the figure). The most notable feature of this gel is that it has three bands, much in the same way as Figure 3.16. This comparison suggests that if the trimer reaction in Figure 3.16 had indeed generated the full trimer structure, there should be four bands, not three. In other words, there should be one more band than we see in Figure 3.34, where two out of three golds are in the structure. This inconsistency motivated us to run the gel in Figure 3.35. ${ }^{16}$ This figure demonstrates branch-2X(AC AAG ATA ACG CAG AGG TAC)-3', sequence "M," 5'-TG CCG ATT ACA CGA ACA TTG-branch-2X(AC AAG ATA ACG CAG AGG TAC)-3', and Sequence "N," 5'-HS-GTA CCT CTG CGT TAT CTT GTT GGA TCG AAG TTA GTA GTC TTA CTT TGC ATG TCA TTC AGG CGT CGT TTC GCG GTT AAG TCG GAT TTC AAG TGA CTG GCA T-3’.

${ }^{16}$ For this gel, the sequences were as follows:

Lane 1, $5 \mathrm{~nm}$ gold with Sequence "H;" Lane 2, the same plus Sequence "J;" Lanes 3 and 8, 5 nm gold with Sequence "A;" Lanes 4 through 6, trimer Sequence "J" and 5 nm conjugates Sequence "A;" Lane 7, conjugates with Sequences "A" and "H" and trimer "J." Lanes 9 through 11 contain conjugates with Sequence "A" and trimer Sequence "K;" Lane 12 contains trimer Sequences "J" and "K" and conjugates Sequence "A." 


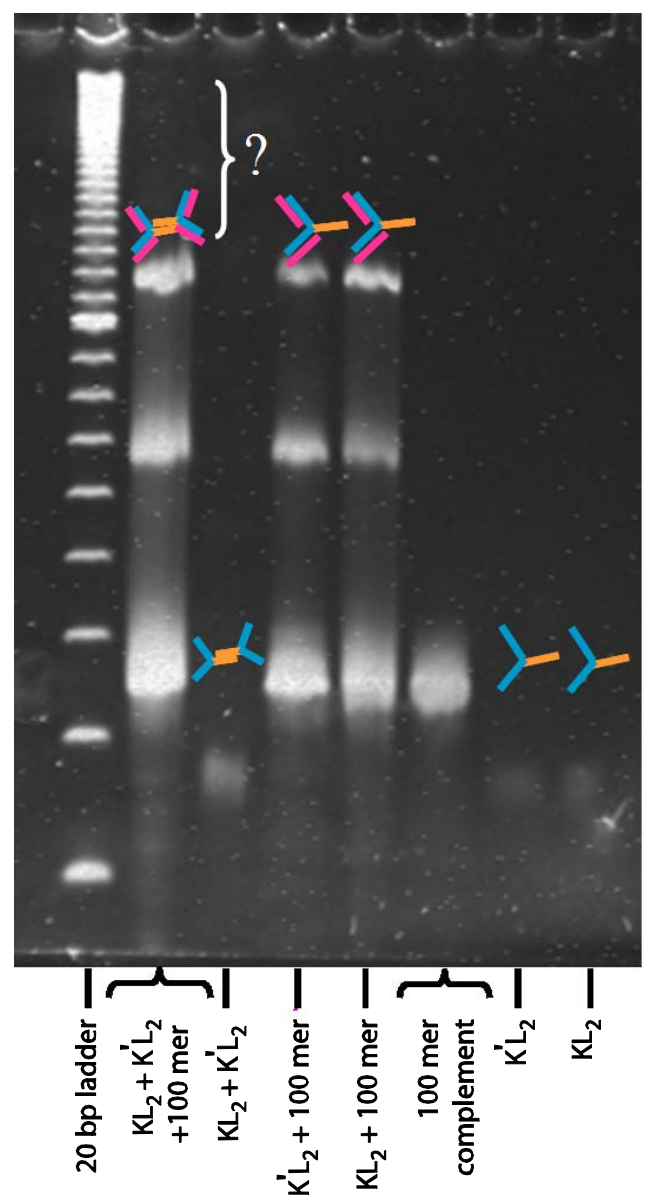

Figure 3.33: $6 \%$ native PAGE gel showing annealing of all portions of the redesigned and synthesized $5^{\prime} \rightarrow 3^{\prime}$ tetramer structure. The lane second from the left contains the entire reaction mixture, but it does not seem to contain any bigger structure other than that of either half, separately. The reason for this problem is unclear. If the two halves had hybridized together, one would also expect to see an upper band in the lane where just the two DNA trimer parts were combined. This gel was run at $4{ }^{\circ} \mathrm{C}$ at $160 \mathrm{~V}$ for about 4.5 hours. The reactions were annealed at a $3 \mu \mathrm{M}$ concentration overnight with $50 \mathrm{mM} \mathrm{NaCl}$. 


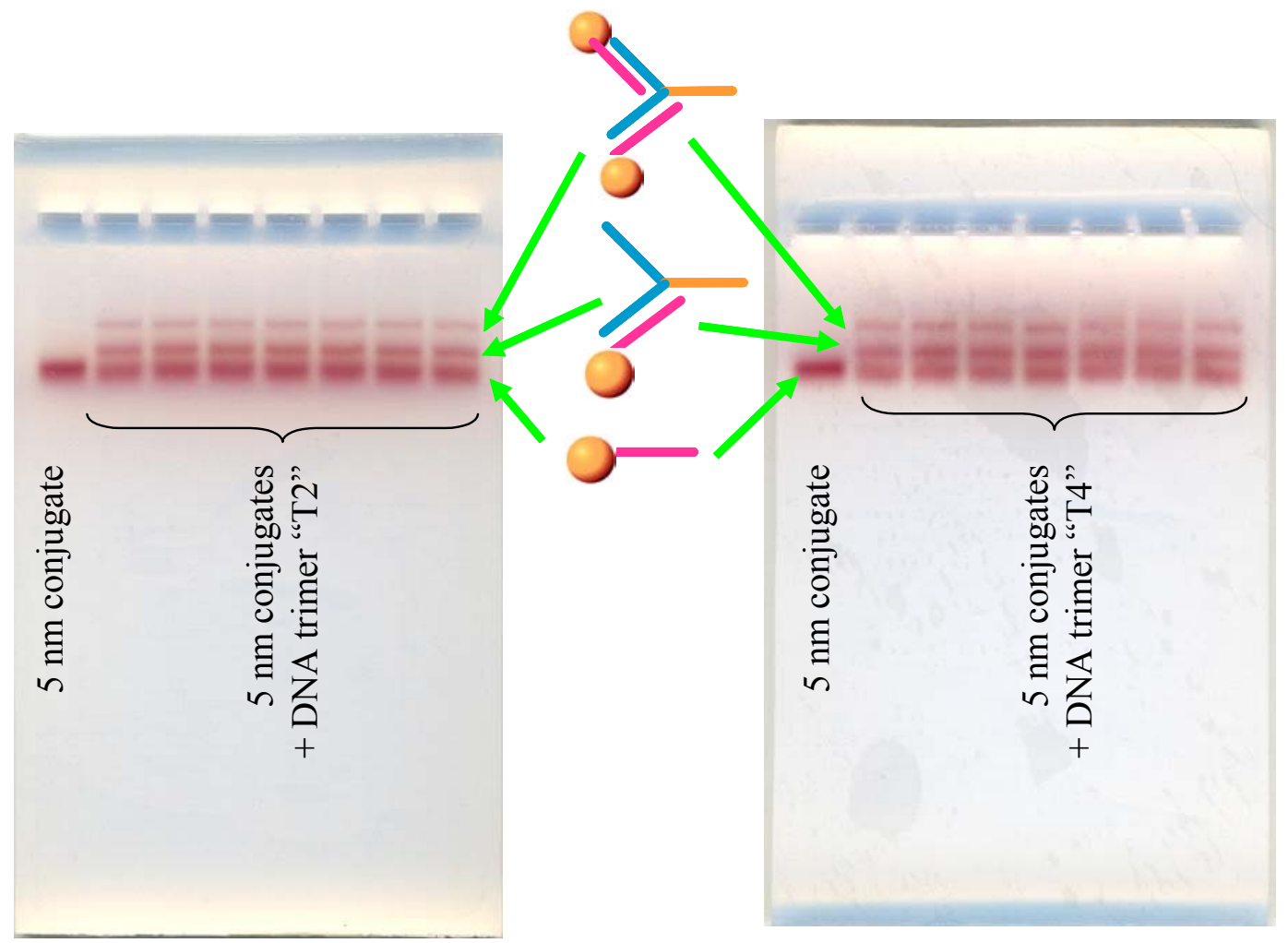

Figure 3.34: $3 \%$ agarose gels showing the result of adding $5 \mathrm{~nm}$ gold-DNA conjugates to separate DNA trimer components of a tetramer structure. The gel on the left contains one trimer half with gold, and the other gel contains the complementary trimer with its hybridized gold conjugates. These reactions were annealed for one day. 


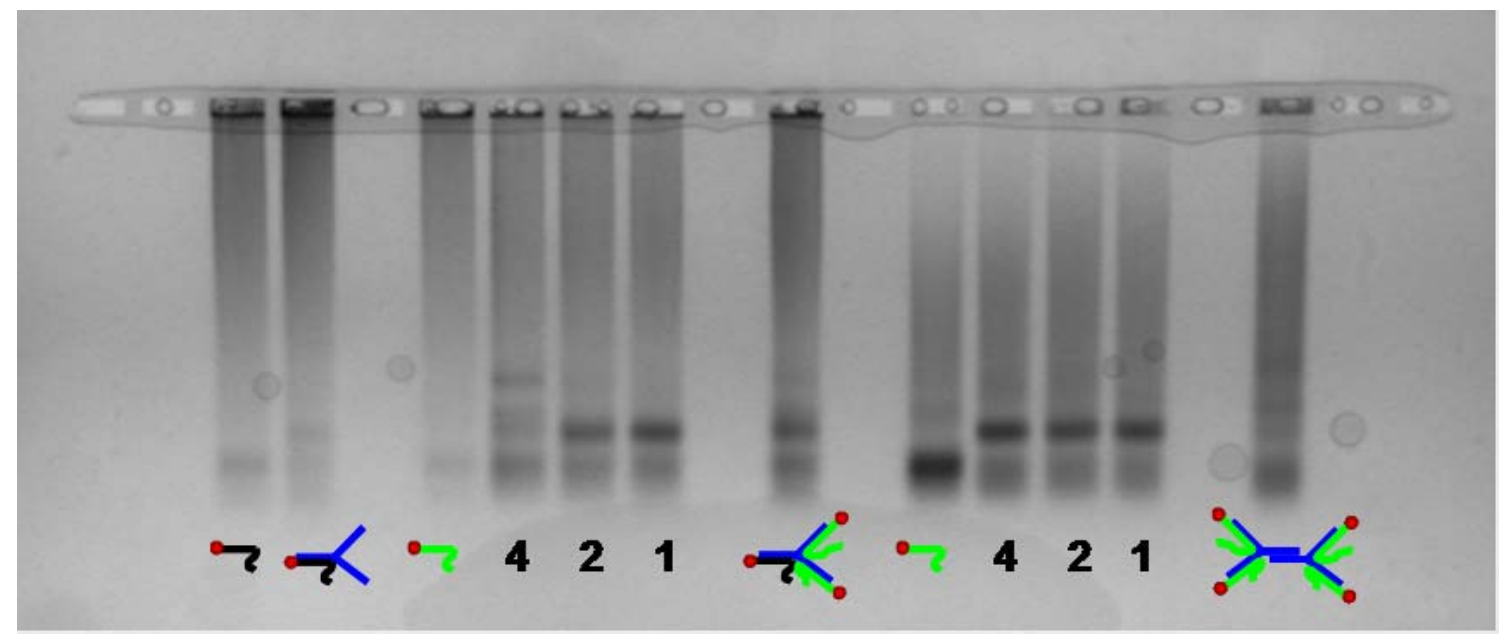

Figure 3.35: 3\% agarose gel, run by Sarah Goh, showing the behavior of trimer and tetramer structures with only some components added. Pictures indicate the possible mosthybridized structure for those lanes. In lanes labeled underneath with numbers, that value is the ratio of conjugate to trimer. Only one type of conjugate has been added in those lanes. Lanes 8 through 11 should show comparable results to Figure 3.34, but they contain one less band. Lane 7 has all the components to make complete trimers, but hybridization ratio was only 1:1. Lane 12 has all the components to make a complete tetramer in a 2:1 stoichiometric ratio. These reactions were annealed overnight. 
that we need more information to conclusively assign the bands in Figure 3.16. At the very least, that experiment would need to be replicated, but all such attempts have either aggregated during preparation, or given inconclusive results.

If we were to use radioactive labeling for this trimer project, we would have a much more sensitive technique for detecting the presence of upper bands in the tetramer or trimer gels, and a greater ability to detect all of the possible bands without having to resort to silver staining that would make the gold samples impossible to isolate. Unfortunately, the resulting gold trimer and gold tetramer samples in those bands would be radioactive, making the next electron microscopy step seem quite difficult to manage! Hopefully improvements that use optical techniques to avoid the use of gels will allow us to avoid band imaging altogether.

\subsection{Project Conclusions}

From these DNA trimer-based experiments, we were able to draw a number of conclusions:

- We can make branched DNA trimers on solid support that hybridize as we expect.

- We can prepare and use single DNA-gold conjugates, and hybridize them to each other, or to the DNA trimer structures

- Preparing the enough of the gold-DNA conjugates to detect the results in an experiment is labor intensive and time-consuming. DNA strand batches and gold nanoparticle batches behave differently between batches during the conjugation step. There 
is significant risk of an isolated single-DNA/gold sample precipitating after isolation.

- Attempts to scale up and improve efficiency of DNA-gold conjugate production need further optimization to work.

- The greatest challenge in this experiment is making enough concentrated, non-precipitated, non-aggregated single DNA-gold conjugates.

- The sample of gold must be concentrated a great deal to allow enough gold to be loaded into the gel sample well for it to be detected visually.

- The more kinds of gold nanoparticles we want to incorporate into a structure, the more bands that may be uniquely present in the gel, and the harder individual bands will be to detect.

- Most of the current gels have not shown enough discrete bands to guarantee the intended structure formed in appreciable quantities. 


\section{Chapter 4}

\section{Progress Toward Using DNA}

\section{Lattices to Pattern Nanocrystals}

\subsection{Motivation}

In order to use nanocrystals as components in a larger system, it would be useful to be able to order or pattern them on a surface. It would be convenient to control both their location relative to each other, and their location relative to particular surface features. Since DNA can be attached specifically to nanocrystals and also localized on a surface, and since it participates in self-assembly, it provides an promising template for assembling surface-based nanocrystal structures. For this project, the goal was to assemble a twodimensional ordered array of nanocrystals having some sort of nanometer-scale spacing using DNA as a template material. 


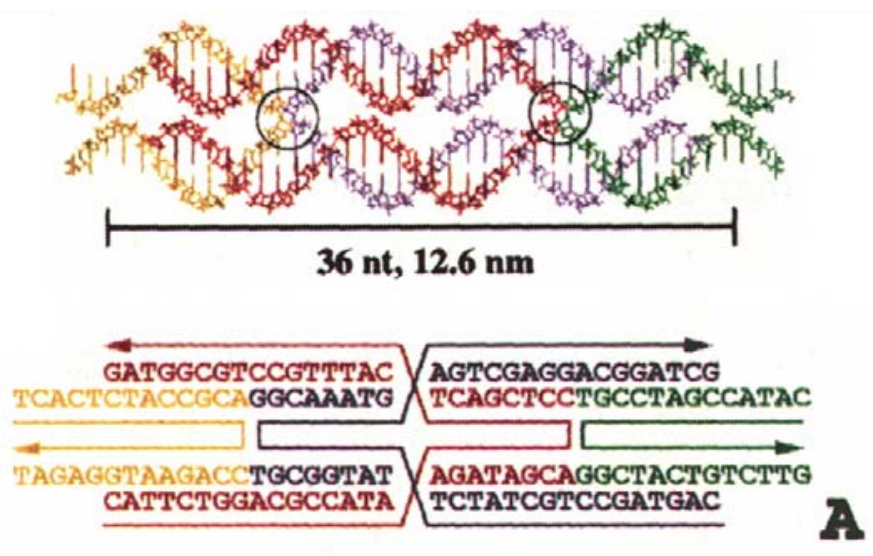

Figure 4.1: Tile "A," one of two tiles in a lattice system reported by Prof. Seeman's group at NYU.[2]

\subsection{Previous Work}

Prof. Nadrian Seeman's research group at New York University developed types of template structures that would facilitate this kind of patterning, [2][70] (one of which we used for these experiments). His group created a rectangular nanoconstruction building block out of DNA called a "tile." This tile was a "double-crossover molecule" comprised of several DNA strands. Figure 4.1 shows one of these double-crossover molecules. The tile is called a "double-crossover molecule" because it contains two DNA junctions, called "crossovers." These junctions are immobilized four-way junctions similar to mobile ones found during genetic recombination, and called "Holliday Junctions."[71] A cartoon of the structure of a Holliday junction is shown in Figure 4.2. $\quad$ Earlier, Prof. Seeman had discovered how to make an immobile junction of this type by eliminating identical sequences (termed "removing sequence symmetry") around a branch point.[67][69] The double-crossover tile structure combined two of these DNA junctions to make a more rigid nanotechnology building block 


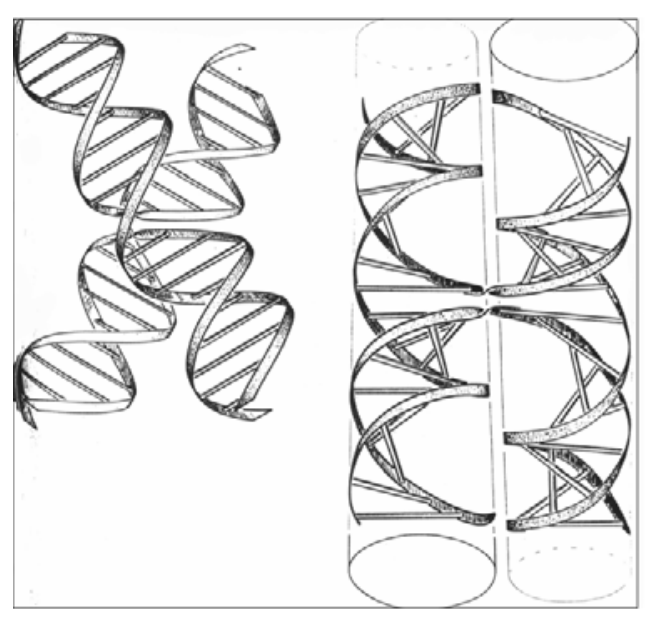

Figure 4.2: Cartoon of the structure of a four-way DNA junction called a "Holliday Junction."[15]

than the junction would be, on its own. Prof. Seeman's research group paired the tile in Figure 4.1 with a second tile, shown in Figure 4.3. These tiles each have corners made of short segments of unpaired DNA that are available to participate in a self-assembly process by forming a double helix with a complementary strand. Tiles A and B bind each other in a catecorner fashion to create a sort of tiled DNA fabric. Figure 4.4 shows the structure of this assembly. Prof. Seeman's group also developed a four-tile lattice system. The tiles for a system of this type are shown in Figure 4.5. If the tiles could be labeled with nanocrystals, the two-dimensional lattice building properties of the tile system could be harnessed to create nanocrystal assemblies, as shown, conceptually, in Figure 4.6.

\subsection{Choice of Lattice System}

The lattice system shown in Figure 4.4 is relatively simple since it is only composed of eight DNA strands, but the four tile system in Figure 4.5 has a method of lattice 


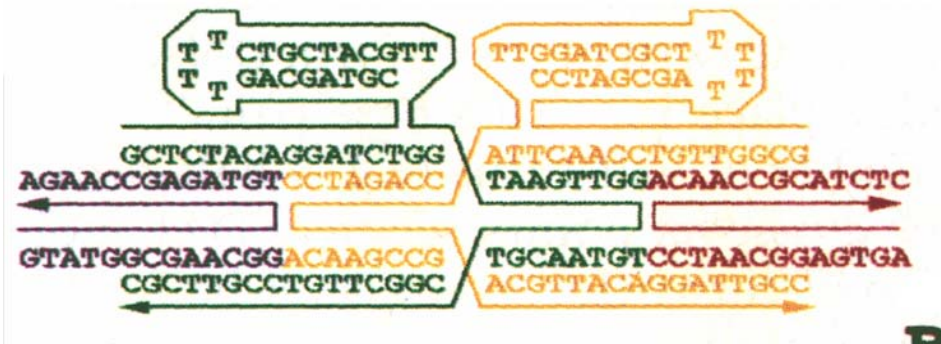

Figure 4.3: Tile "B" complement to "A" in Figure 4.1. Both Tiles A and B have four "sticky ends" apiece. Tile B has two hairpins. Because of the helix turns not pictured in this cartoon, one of the hairpins sticks up from the surface of the tile, and the other sticks down in the opposite direction.[2]

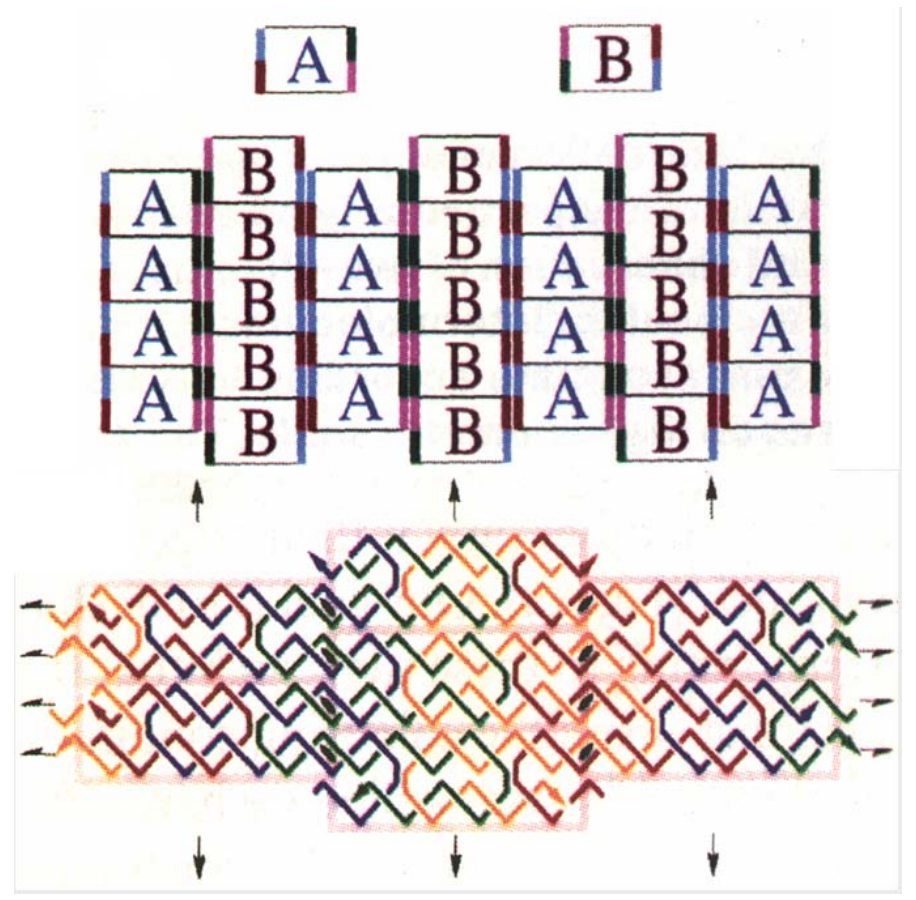

Figure 4.4: Tiles A and B have sequences designed to complement each other at their corners. Self-assembly leads to two-dimensional DNA array. The array has a periodicity of $25 \mathrm{~nm}$ from the stripes formed by the hairpin bumps coming from the top of Tile B.[2] 


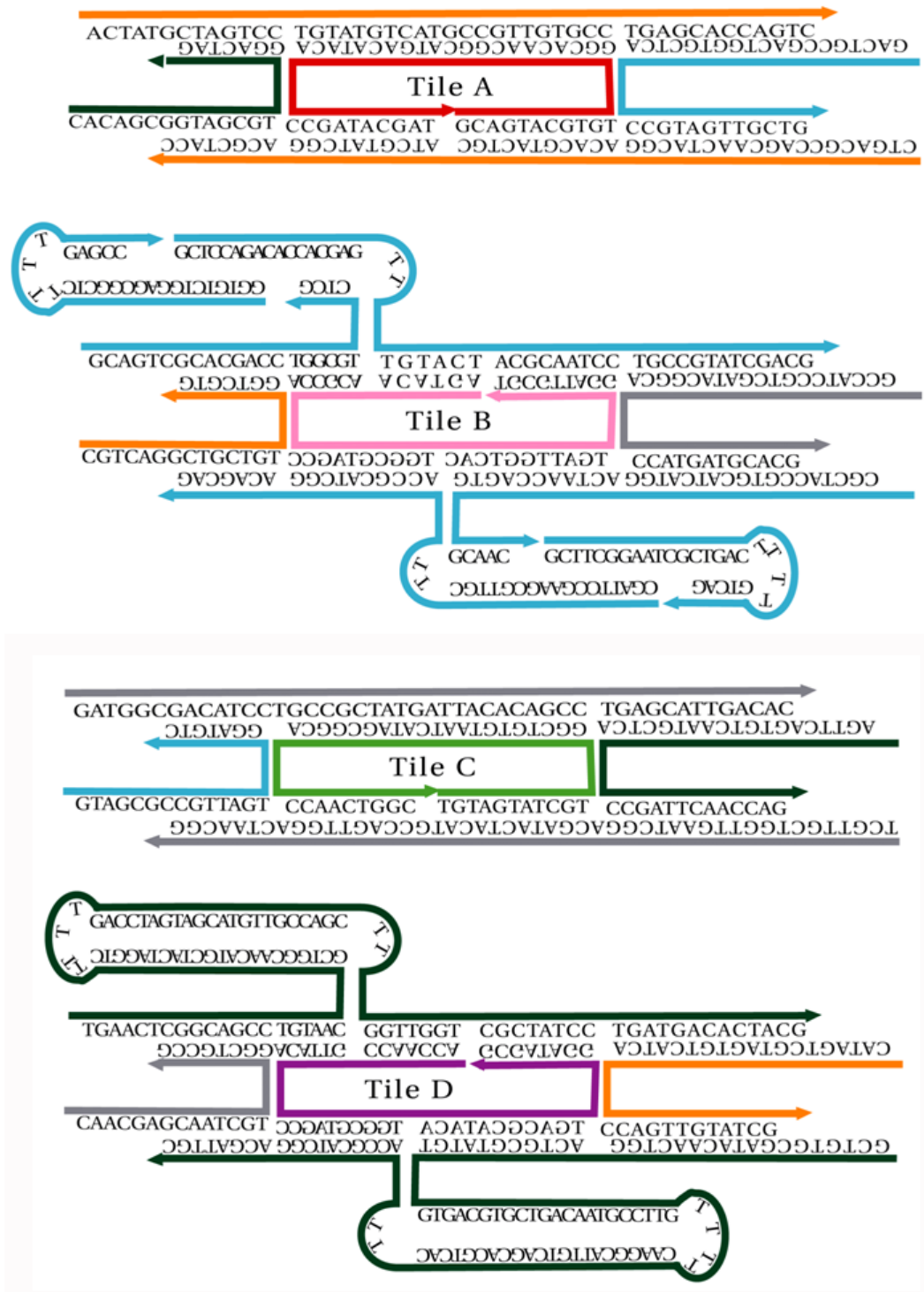

Figure 4.5: Tile components for a four-tile lattice system. Tiles B and D have hairpins that stick up above the resulting lattice fabric. Tile B's hairpins are nicked so the hairpin strand can be included or not as wished.[3] 


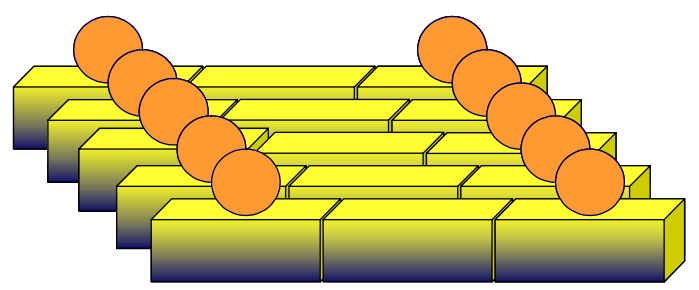

Figure 4.6: Figure showing rectangular tiles labeled with nanocrystals, combined with an set of unlabeled tiles to form a nanocrystal array on top of a two-dimensional lattice structure.

modification built into its structure in Tile B, as you can see in Figure 4.5. Two strands in Tile B make most of the two hairpins, and these locations can be used to label the lattice structure with nanocrystals. A cartoon of this scheme is shown in Figure 4.7. Unfortunately, the four-tile $\mathrm{ABCD}$ lattice system is comprised of many more DNA strands than the A-B lattice in Figure 4.4: 24 strands, to be exact.

\subsection{Lattices and Nanocrystal Compatibility}

The lattices anneal to form Watson-Crick double helices, so the same $\mathrm{pH}$ and salt conditions that enable normal double helices to form would be expected to work for this system. A neutral to slightly basic $\mathrm{pH}$ works well, along with sodium ions to shield the negative charges of the DNA backbone. For the double-crossover molecules that form the tiles, however, each have two Holliday-junction-like crossover points. The Holliday junctions, because of the very close proximity of negative charges from the DNA backbone at the crossover point, require the presence of divalent magnesium ions in the annealing buffer for the junctions to form successfully.

The need for magnesium ions in the DNA lattice annealing buffer creates severe complications for an experiment designed to incorporate nanocrystals. Gold nanoparticles 

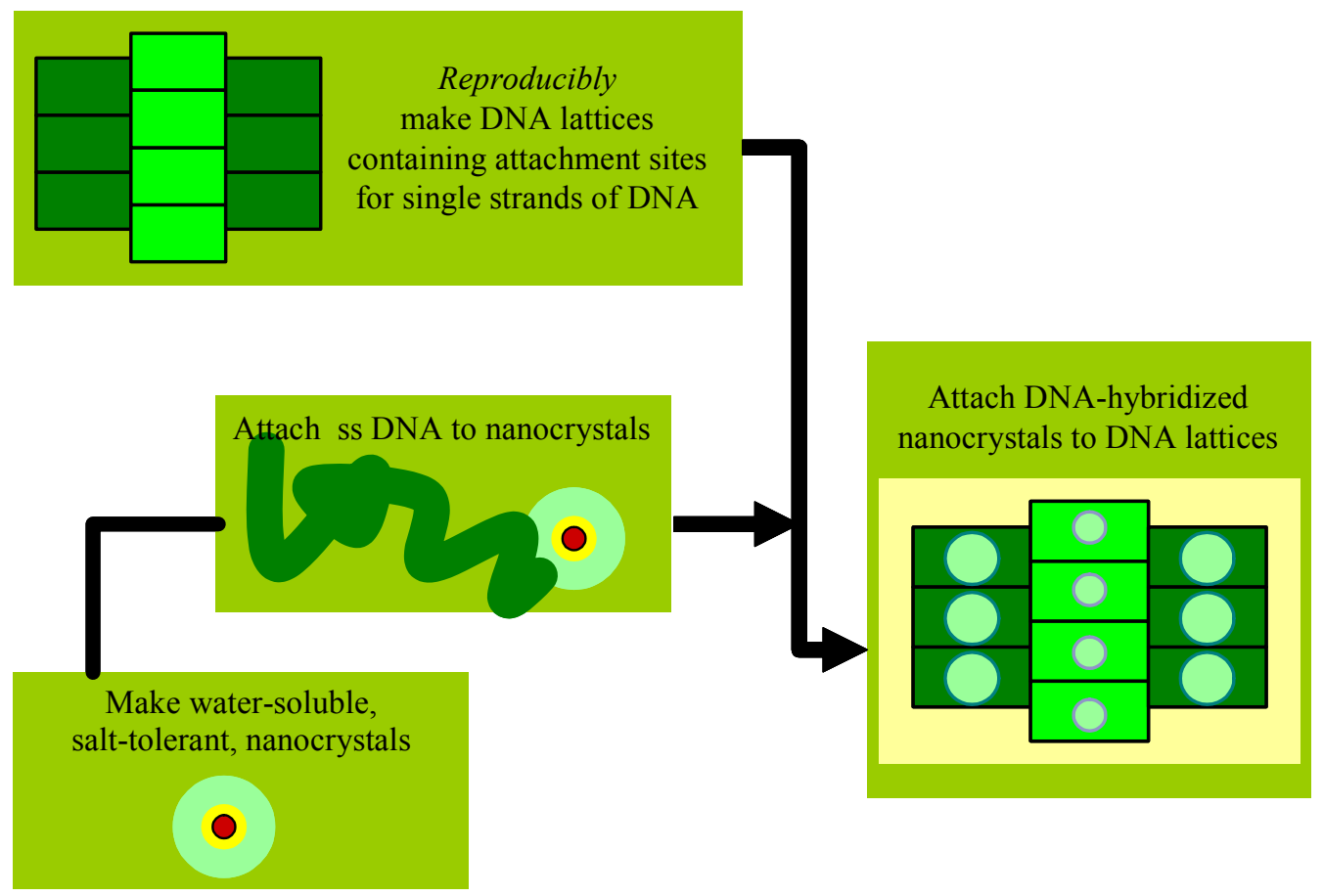

Figure 4.7: A scheme of the experiment plan for making ordered arrays of nanocrystals using DNA lattices as scaffolding. 
are charge-stabilized, and the divalent magnesium ions cause them to aggregate, even if they are stable in the presence of sodium.

For the previously published lattices, Prof. Seeman's research group used 6-10 $\mathrm{mM} \mathrm{MgCl}_{2}$ in the buffer for annealing. We used only 50-100 $\mathrm{mM} \mathrm{NaCl}$ for annealing gold dimers and trimers, and no magnesium at all. The main challenge for this project was to find conditions compatible for both retaining lattice structure and preventing nanoparticle aggregation. At the conclusion, though, we did not find conditions workable for both.

\subsection{Making Lattices}

The procedure for making lattices began with preparation of the twenty-four DNA strands necessary to create the four tiles in Figure 4.5. The twenty-four strands were all unique, and ranged in size from 21 to 98 bases in length. It was not possible to order the strands in a sufficiently pure form at any price from any company we could find. The presence of even the smallest amount of impurities in a batch would prevent the lattices from properly assembling. The standard for rigorous purity for these strands was far and above the standards of purity for any biological experiments using DNA. Most commercial suppliers anticipate only a biological purpose for their product in their preparation process. As a result, we ordered the DNA strands only desalted, which removed protection groups from the synthesis process, but not much else. ${ }^{1}$ We then loaded many denaturing PAGE gels with each strand to resolve and remove any contaminants of a different molecular weight or structure. There is an array of these gels pictured in Figure 4.8.

\footnotetext{
${ }^{1}$ Almost all of our strands were supplied by Integrated DNA Technologies, Inc. A few very early strand batchs, however, were procured from Sigma-Genosys.
} 

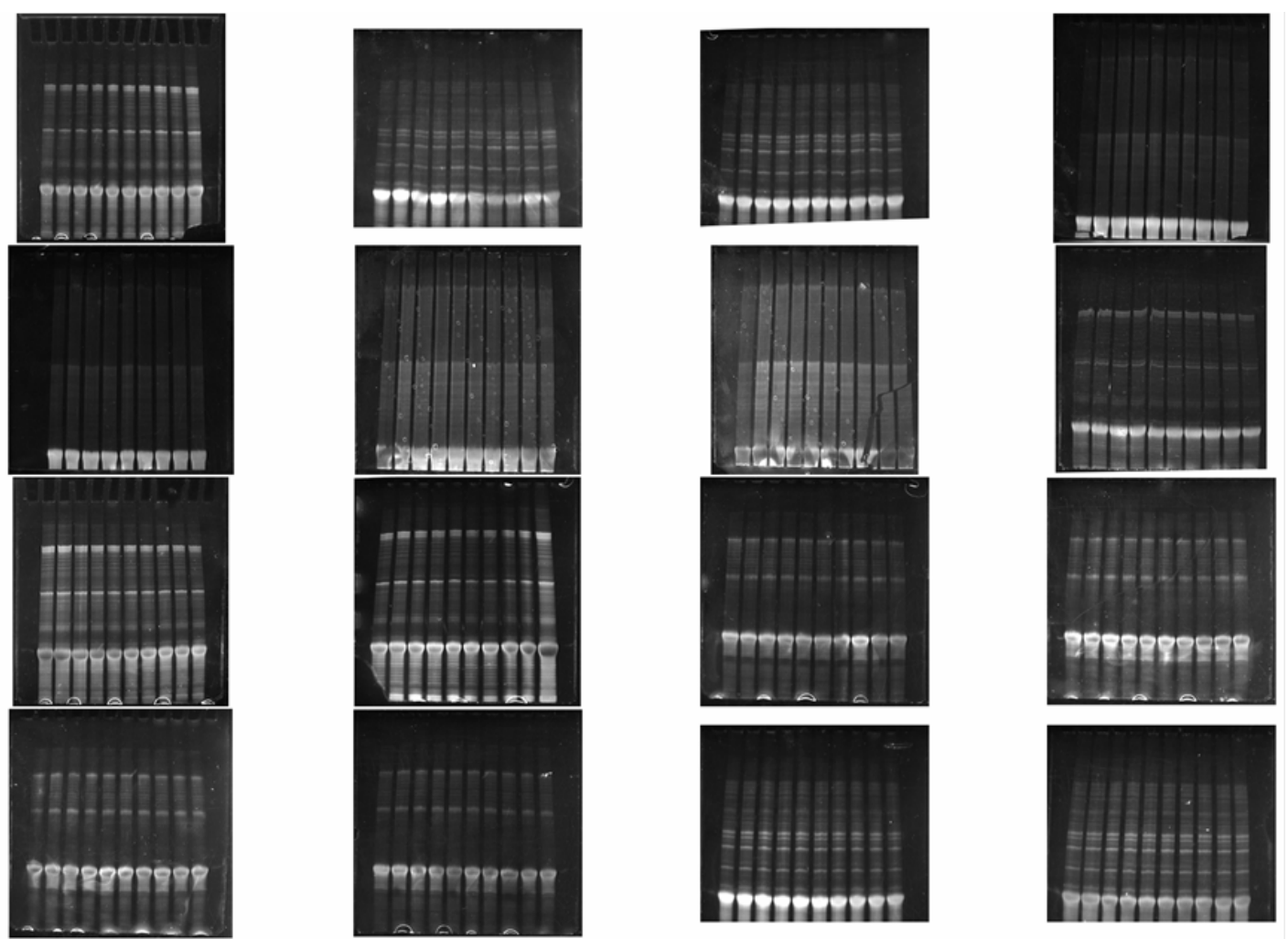

Figure 4.8: Either 10 or $15 \%$ denaturing PAGE gels purifying various strands out of the 24 necessary to prepare a four-tile lattice system. The identity of the strand in any particular gel in the image is not of interest here. The point is to merely give an overview of how such a set of gels would appear. This would not be nearly a sufficient number of gels to prepare all of the lattice strands. Usually, we needed 4 or more gels of any particular strand to yield enough product to do experiments. The result was that 100 or more gels were necessary to produce enough DNA to make a set of lattices. 
The DNA prep gels entailed a substantial amount of work, and the tasks themselves were tedious and repetitive. It really is difficult to overestimate the labor-intensiveness of this procedure. With three gel boxes, a reasonably full, productive day would yield twelve prepared, run, stained, and extracted gels. With a doubling plate in all possible locations (a later improvement in our lab, not used by Prof. Seeman's lab), as many as twenty or thirty could be run in a long, busy, 15-hour day, or with two competent people (as we had for the DNA trimer project). ${ }^{2}$ With so many gels running, a backlog would develop for staining, though, so twelve in a day was more of an achievable norm for an eight-to-twelve hour day for a single person, or even two. Preparing a set of gels took 45 minutes to 2 hours to pour and polymerize. Assembling and loading a gel box took 15 minutes to a half-hour. Depending on the percentage of the gel (longer strands required a lower-percentage gel to reduce run time), a loaded gel box would take two to five hours to run. Developing a gel to stain the DNA so we could see it took thirty minutes, and imaging and extracting the purified DNA bands took thirty minutes. The result was that running a single gel took four to nine hours, making it a time-consuming task to run more than 100 gels.

After purifying all of the DNA lattice strands according to the procedure in Section 2.3.2, it was necessary to load the purified samples into new gels to check that they were indeed pure. With twenty-four different strands, it was imperative to forestall the distinct possibility that any strand had been insufficiently purified the first time, or that even two strands were accidentally mixed during the extraction process, which required many vials to be kept separate and correctly labeled over the course of several days. A sample of

\footnotetext{
${ }^{2} \mathrm{~A}$ doubling plate was a thin glass plate with a depression cut along one edge to allow the buffer to make good contact. It could be inserted into the middle of a gel sandwich so that a single gel box could hold a maximum of four gels, rather than just two.
} 
this type of purity check for the lattice strands was shown earlier in Figure 2.10. Lanes containing insufficiently pure DNA would show faster bands corresponding to incremental omission of one or more bases in the strand.

Once the DNA was indeed pure (and dry), we added sufficient water to create (approximately) a $20 \mu M$ solution. We then quantitated each solution by absorption at $260 \mathrm{~nm}$ to determine the exact concentration of the DNA. We could individually calculate the extinction coefficient for each strand for use in these calculations. ${ }^{3}$ Because of the substantial error in pipetting small quantities of liquid (as much as 10\%), the solutions were rarely exactly $20 \mu M$. We calculated the amount necessary for a stoichiometric addition of each strand to make $50 \mu \mathrm{l}$ of a $0.4 \mu M$ solution ( for 20 pmol of lattice), mixed these amounts (always adding volumes somewhere between $0.5 \mu \mathrm{l}$ to $1.5 \mu \mathrm{l}$ per strand), and then added 5 $\mu \mathrm{l}$ of 10X TAE buffer with magnesium added. ${ }^{4}$ This mixture was place in a siliconized 1.5 ml Eppendorf tube for annealing.

In the initial stages of this project, we expended quite a bit of effort trying to replicate these lattices, as published by Winfree, et. al.[2] The lattices did not form as we had hoped. After going to New York to learn directly from Prof. Seeman's research group, we concluded that the main explanation for this problem (although there were a few) was probably our use of insufficiently pure DNA. Prior to our stay in New York, we had always ordered our DNA strands directly from the commercial supplier already purified, and we did not realize that the success of the experiment was entirely predicated on the strict purity of the DNA. Instead, we imagine that the problems stemmed from not sufficiently controlling

\footnotetext{
${ }^{3}$ We found IDT's "OligoAnalyzer" on their website (http: \\www.idtdna.com) to be useful for this purpose. It can be done by hand by merely counting the number of bases of each type in the strand, multiplying the extinction coefficient for each base by the number of those bases, and then summing.

${ }^{4} 10 \mathrm{X}$ TAE $/ \mathrm{Mg}^{2+}: 400 \mathrm{mM}$ Tris Acetate, $110 \mathrm{mM} \mathrm{Mg}(\mathrm{Ac})_{2}$, and $10 \mathrm{mM}$ EDTA.
} 
the exact stoichiometric ratios of the various strands. Having too many of any particular strand did not seem to be significant, in the end, after all. Pipetting routinely could be expected to introduced as much as $10 \%$ error into the stoichiometric control, especially in the reaction involving 24 different strands. That did not seem interfere fatally with the lattice formation. We did not, however, explore the lattices' toleration for the pipetting error in any sort of extensive or quantitative way.

Another possible source of trouble during our initial attempts to make lattices could have come from our use of non-siliconized Eppendorf tubes. The siliconized tubes discouraged water and DNA from sticking to the tube walls. If lattices formed under these circumstances without a silicon coating, they could have adhered to the walls, and then not have been available for deposition.

During this initial period of lattice experiments, we were able to get the individual Tiles A and B for the two tile system to form completely in gels. Figures 4.9 and 4.10 are tile formation gels that show addition of increasing tile components until the all of the components of the tile give a higher band that corresponds to the complete tile structure.

(These two tiles combine to form the eight-strand lattice in Figure 4.4.)

After mixing all of the strands to make lattices, we annealed them. Normally, we mixed stoichiometric amounts of all of the strands all at once, and annealed them as a batch. Lattices would also form if you first annealed the strands for each tile separately, and then mixed the tiles and annealed again. The annealing, as reported in the original papers, $[2][3]$ took place by placing the vials in a beaker of $2 \mathrm{~L}$ boiling water, and then placed the beaker inside a styrofoam box with a lid. ${ }^{5}$ For our experiments, we used a styrofoam

\footnotetext{
${ }^{5}$ The styrofoam box was the type commercial suppliers typically use to ship refrigerated or frozen bio-
} 


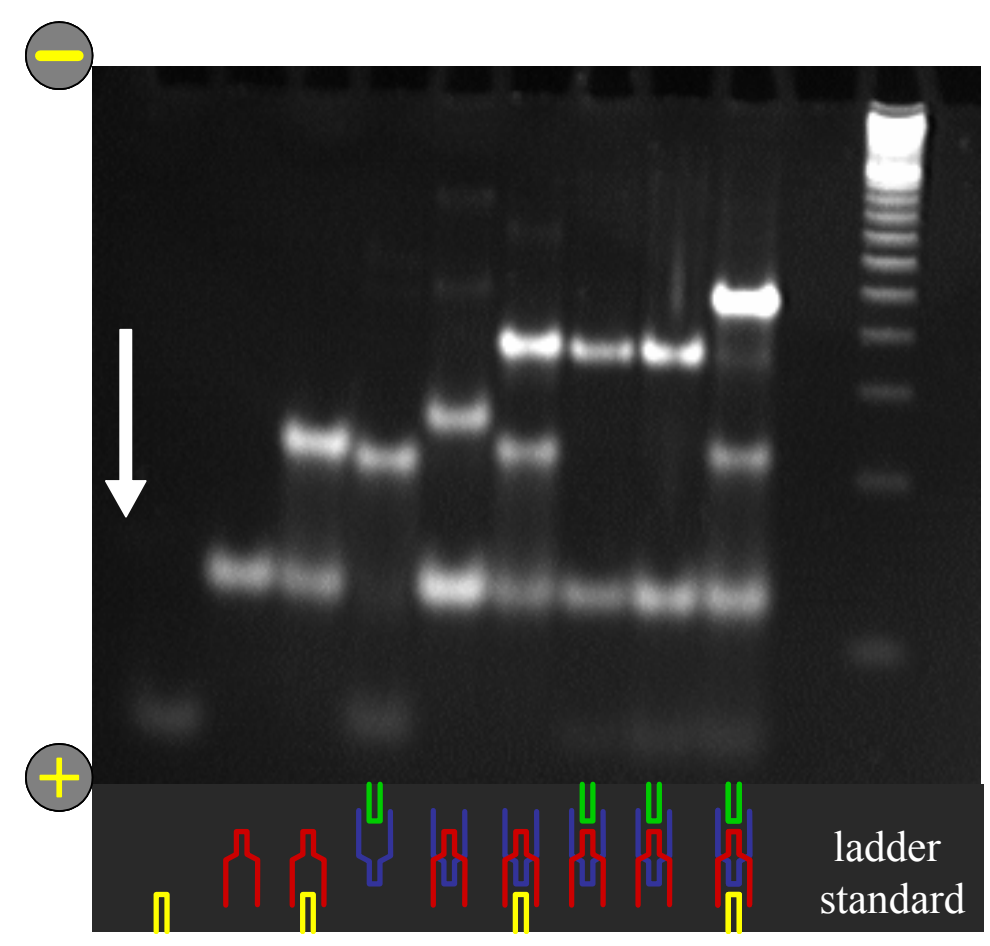

Figure 4.9: $12 \%$ native PAGE gel showing the formation of Tile A. The yellow and green strands are each 26 bases long, and the red and blue strands are 48 bases long. The highest band in the second lane from the right, with all four strand components, contains complete double-crossover tiles. This gel was run in $1 \mathrm{X}$ TAE with $12.5 \mathrm{mM}$ magnesium acetate and $3 \mathrm{mM}$ sodium acetate, recirculated at $300 \mathrm{ml} / \mathrm{min}$. at $4 \mathrm{~W}$ constant power for $15.5 \mathrm{hrs}$. The tile anneal was $1.9 \mu \mathrm{M}$ in all strands, and in a magnesium-containing buffer. 


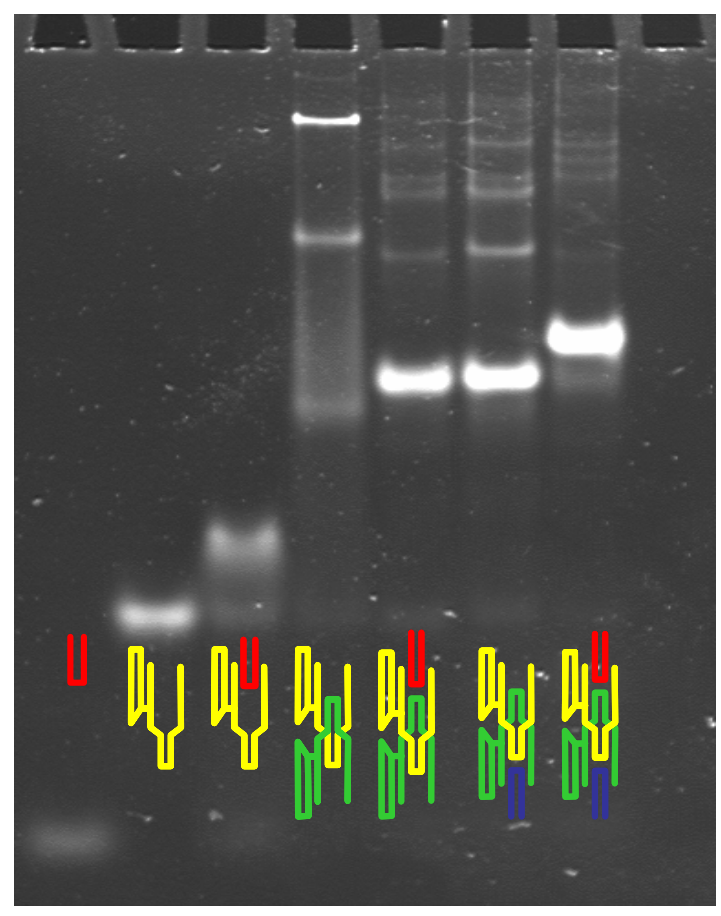

Figure 4.10: $8 \%$ native PAGE gel showing the formation of Tile B. The red and blue strands are each 26 bases long, and the green and yellow strands, which have hairpins, are 70 bases. This tiles components gave complicated, highly retarded structures as soon as the complementary 70 base strands were both present, and the complete tile formation was in the brightest band on the right-hand lane, not the upper, lighter bands. We do not know what structures were in those uppermost bands. This gel was run in 1X TAE with $12.5 \mathrm{mM}$ magnesium acetate and $3 \mathrm{mM}$ sodium acetate, recirculated at $300 \mathrm{ml} / \mathrm{min}$., for $3 \mathrm{hrs}$. at $80 \mathrm{~V}$ and $7 \mathrm{hrs}$. at $100 \mathrm{~V}$. The tile was annealed at a $1 \mu \mathrm{M}$ concentration in magnesium-containing buffer. 
box like this with a floating vial holder, or, alternately, a piece of thermal cookware called a "Shuttle Chef." 6 To make lattices, we annealed our reactions for at least two days. The lattices formed in solution, not on a surface.

We tried a variety of schemes to make lattices that were modified with gold nanocrystals. A flow chart describing these schemes is portrayed in Figure 4.11. Most of the possible variations in this process revolve around a decision about how much purification to do on the gold nanocrystals and lattices before and after addition to the lattices. In the simplest case, we could conjugate the DNA to the gold, separately make the lattices, add the gold-DNA conjugates to the lattices, and then deposit the modified lattices and image them.

Alternately, we could conjugate the DNA to the gold, isolate the single-DNA conjugate (provided the DNA was longer than required by the lattice design, to allow resolution of discrete conjugates), and then add those to the lattices. We also had to decide whether to add the gold to the lattices before or after the lattices were deposited for imaging. ${ }^{7}$ One problem with adding gold after deposition was that the solution for surface modification of lattices required the presence of a mixture of $30 \%$ isopropanol with buffer to prevent the lattices on the surface from redissolving while the modification reaction was

logical samples. It was big enough to hold the beaker completely. In our initial attempts to replicate the lattice experiment, this information was non-trivial. . . .

${ }^{6}$ The "Shuttle Chef" thermal cookware consisted of an outer, vacuum insulated jacket with a lid, similar to a coffee Thermos. An inner, clad metal 18-8/carbon steel pot could be separated from the outer jacket to boil $2 \mathrm{~L}$ of water, and then placed inside the insulated outer jacket to allow a very slow cool of the contents inside. The Shuttle Chef was manufactured by Thermos/Nissan (Schaumburg, IL).

${ }^{7}$ To deposit lattices for imaging, $5 \mu \mathrm{l}$ of annealed lattices were placed on a freshly cleaved mica substrate under a petri dish cover in a humid atmosphere (created by putting a surrounding puddle of water) for 3 min. The humid atmosphere prevented uneven deposition caused by evaporation of the deposited lattice solution. After $3 \mathrm{~min}$., the mica substrate was removed, rinsed gently with a couple of drops of water, and then dried completely with canned compressed "air" in an extremely gentle way. 


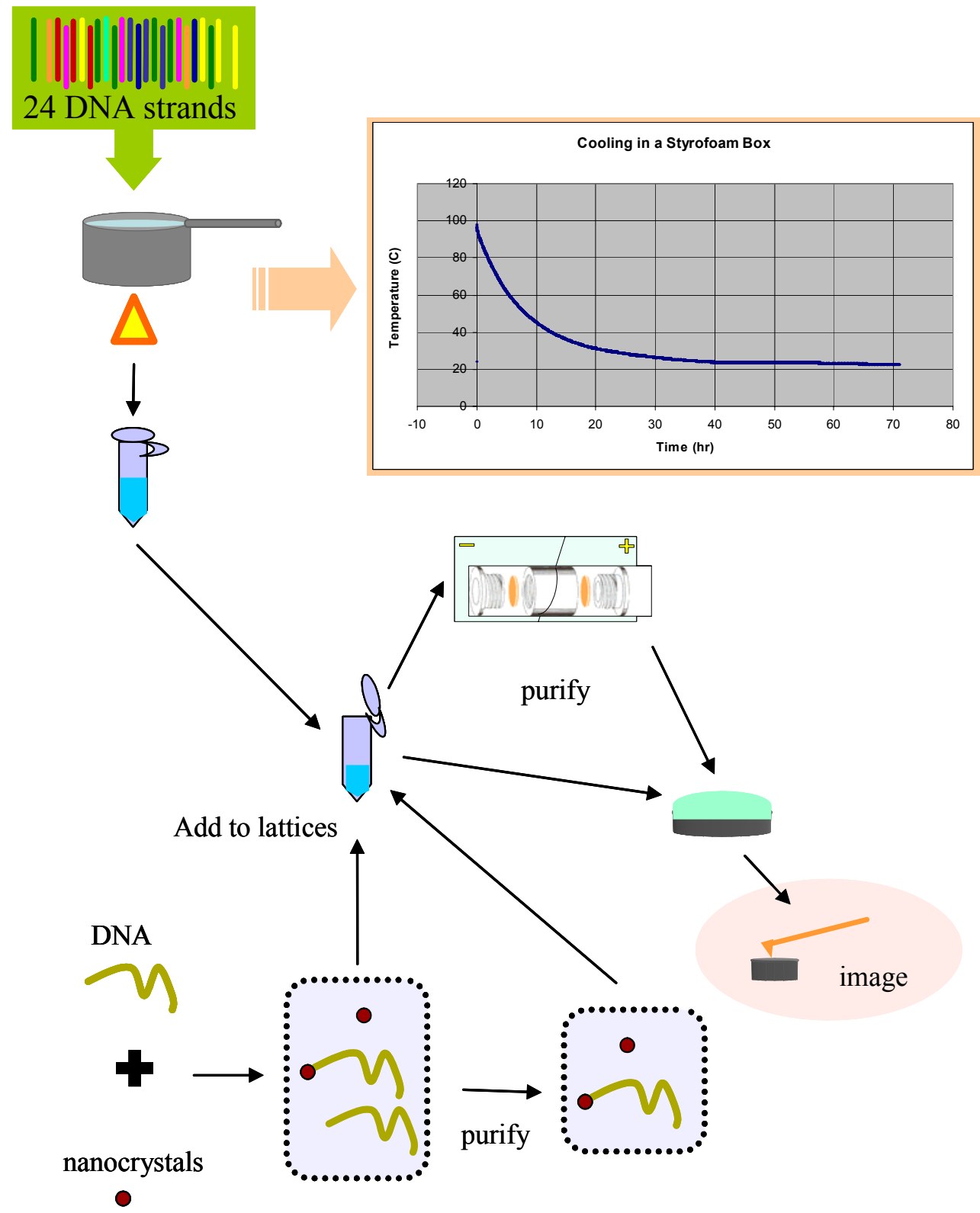

Figure 4.11: Schematic showing the process for making and modifying DNA lattices with gold nanocrystals. The thermal profile on the top right of the figure is actual data of the temperature versus time for an annealed reaction floated in $2 \mathrm{~L}$ of boiled water in a beaker inside a styrofoam box. 
taking place. ${ }^{8}$ This isopropanol mixture was not a stable solution for the gold nanocrystals.

Once we realized that the nanocrystals were adding to the lattices in a non-specific way (in addition to any specific binding at the designed hairpin addition sites) we introduced an electrodialysis step to the procedure. Electrodialysis allows electrophoretic separation of charged samples by placing them in a tiny chamber, bathed in buffer, with membranes that allow samples smaller than a cutoff size to pass through. The electrodialysis unit we used had membranes available with molecular weight cutoffs of 100 Daltons up to 1,000,000 Daltons. A picture of the setup is shown in Figure 4.12. As shown in Figure 4.11, electrodialysis in this setup was a method we tried in order to remove non-specifically bound DNA and nanocrystals from the lattices before imaging.

\subsection{Characterizing Lattices}

We characterized the DNA lattices using atomic force microscopy (AFM). The atomic force microscope is a form of "scanning probe microscopy" invented in 1986 by Binnig, Quate, and Gerber.[72] Figure 4.13 contains a diagram with an explanation showing how an atomic force microscope works. The horizontal resolution of the AFM (both the X and Y directions) is primarily limited by the shape of the tip. The resulting topographic images are actually a convolution of the tip and sample shapes. AFM tip shapes vary with the identity of the individual tip. For small samples, like DNA, which has a diameter of 2

\footnotetext{
${ }^{8}$ Surface modification of lattices proceeded smoothly for DNA hairpins not attached to gold when the mica substrate with dried lattices was placed on an island of an inverted petri dish in a puddle of $30 \%$ isopropanol inside a recrystallization dish, covered with parafilm. 10 pmol of the hairpin complements to Tile B in the four lattice system were placed in $30 \mu \mathrm{l}$ of $30 \%$ isopropanol in buffer on top of the deposited lattice sample. The overlay was left for $30 \mathrm{~min}$. at $4^{\circ} \mathrm{C}$ for $30 \mathrm{~min}$. Then the mica was again gently rinsed and dried (still in the cold room). This procedure never worked for gold nanocrystal-containing solutions because the nanocrystals aggregated in the isopropanol-containing buffer.
} 


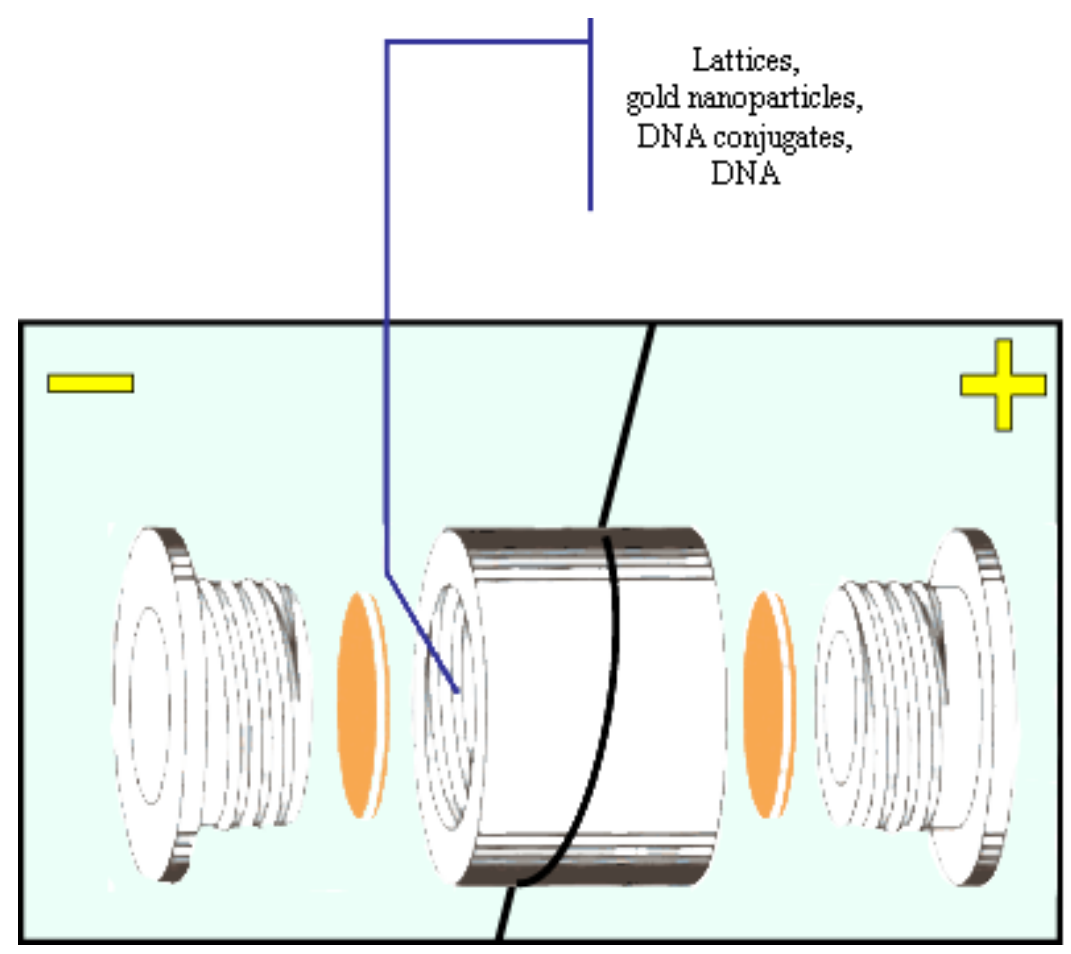

Figure 4.12: Diagram of electrodialyzer setup supplied by Harvard Apparatus (Amika). A teflon chamber with screw-on teflon caps created a sample chamber. The teflon caps were hollow, as shown in the picture. They affixed membranes (of a type and size chosen by the user) on both sides of the chamber, allowing the internal sample to make electrophoretic contact with the surrounding buffer. The portion of the sample smaller than the membrane's MWCO leaves the internal chamber after only 5 or 10 minutes. Additional chambers could be screwed to the first to trap samples of different sizes behind different membranes, if desired.[16] 


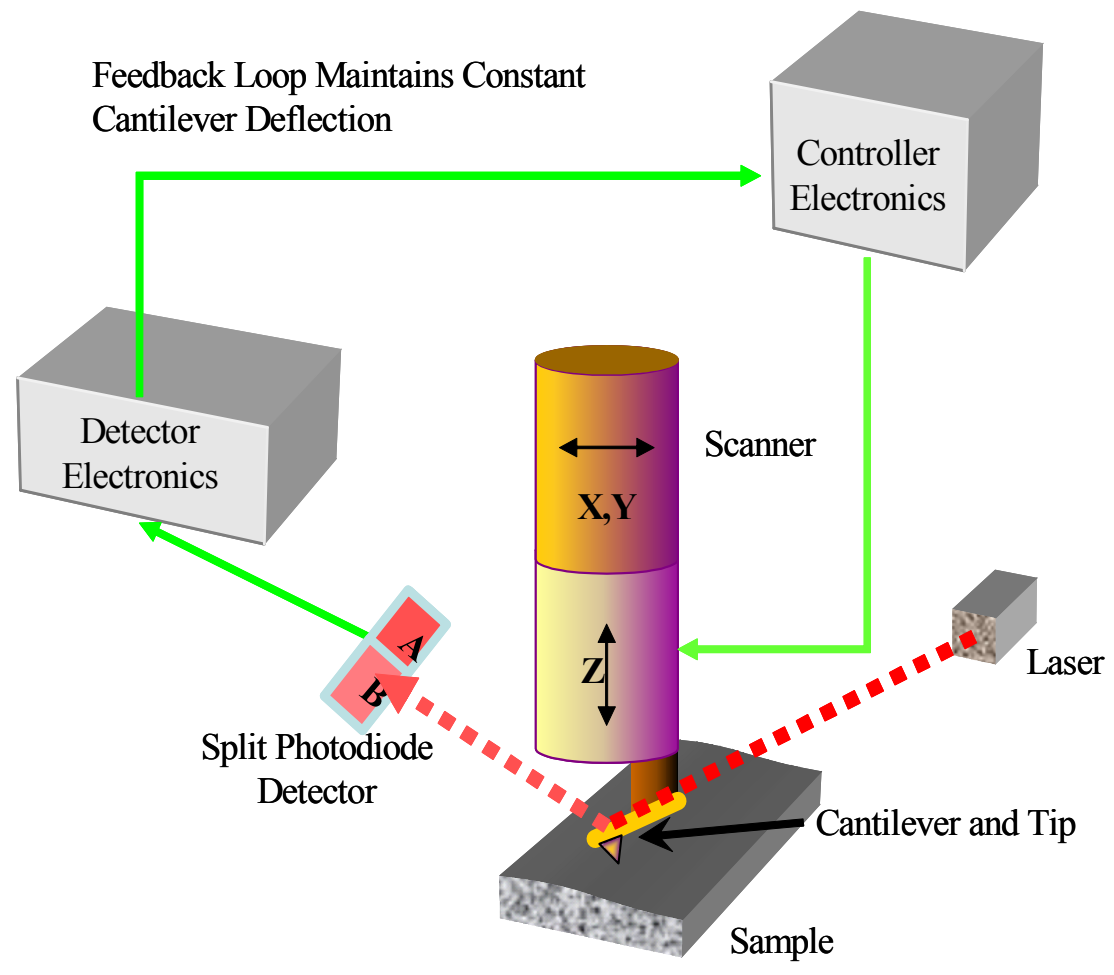

Figure 4.13: Diagram showing how an atomic force microscope works. Very roughly, it works similarly to a record player, but on a much smaller scale. A probe with a tip with a radius of less than $10 \mathrm{~nm}$ fabricated from a silicon or silicon nitride wafer is scanned across the sample surface. A piezocrystal controls the vertical and horizontal position of the tip relative to the surface. The back of the probe is reflective, and a laser bounces off of the back of the probe onto a split photodiode detector. The position of the laser light reflection on the photodiode tells the detector electronics the angle of the bend of the cantilever, which is affected by the surface features. This information allows the controller electronics to adjust the height of the probe, using the piezocrystal, to reflect the surface topography, and the computer records the height information as it rasters across the surface to create a computerized topographic image. A variation on this process vibrates the probe cantilever near its resonant frequency, and the detector records dampening of its oscillation, allowing the controller to adjust height with that information to keep the amplitude constant. The advantage of this method, (called "tapping mode"), is that the tip does not, then, directly drag across the surface. Dragging can damage a softer sample. 
$\mathrm{nm}$, the effect of the size and shape of the tip becomes quite substantial. The result for DNA or nanocrystal-sized samples is that they appear to be much wider and fatter than they really are. The vertical resolution ( $\mathrm{Z}$ direction) is much better, though, and can be about $1 \AA$.

Our interest in the AFM primarily centered around its ability to image DNA. Several techniques have been developed for imaging DNA on a surface by AFM. Two methods we used included imaging dried DNA samples under alcohol[73], and in buffer through cation-mediated absorption.[74] The main reason to image DNA under a fluid overlay is to reduce the forces between the tip and sample. Without the fluid overlay, water condenses at the tip-sample interface due to the effect of capillary condensation. The condensed water drags across the sample with the tip, increasing the shear forces on the sample. With the alcohol or buffer overlay, the fluid prevents capillary condensation.

We could achieve good quality images with either method. Figure 4.14 shows an image of plasmid ${ }^{9}$ DNA taken (in contact mode) under n-propanol on a mica substrate.[73] This type of DNA, from a natural source, is almost two orders of magnitude longer than the DNA we used for our lattice and trimer experiments. The DNA is visible as long threads in the image. The images show supercoiling of the plasmids. ${ }^{10}$ We also got even better plasmid images using a buffer overlay method, which allowed the DNA sample to avoid being dried on the surface, presumably preserving a more natural environment. Figure 4.15 shows two plasmid DNA images collected by nickel cation-mediated sample deposition.

\footnotetext{
${ }^{9} \mathrm{~A}$ plasmid is a circular ring of DNA usually found in bacteria that is not part of the main chromosome. The specific identity of the plasmids here was not so important here, so we used convenient samples supplied by other people.

${ }^{10}$ Supercoiling is a property of DNA unique to plasmids. Because the plasmid is a closed loop of doublestranded DNA, it is possible that the loop contains a number of additional twists (supercoiling) before the two ends of it were joined to make a single ring.
} 


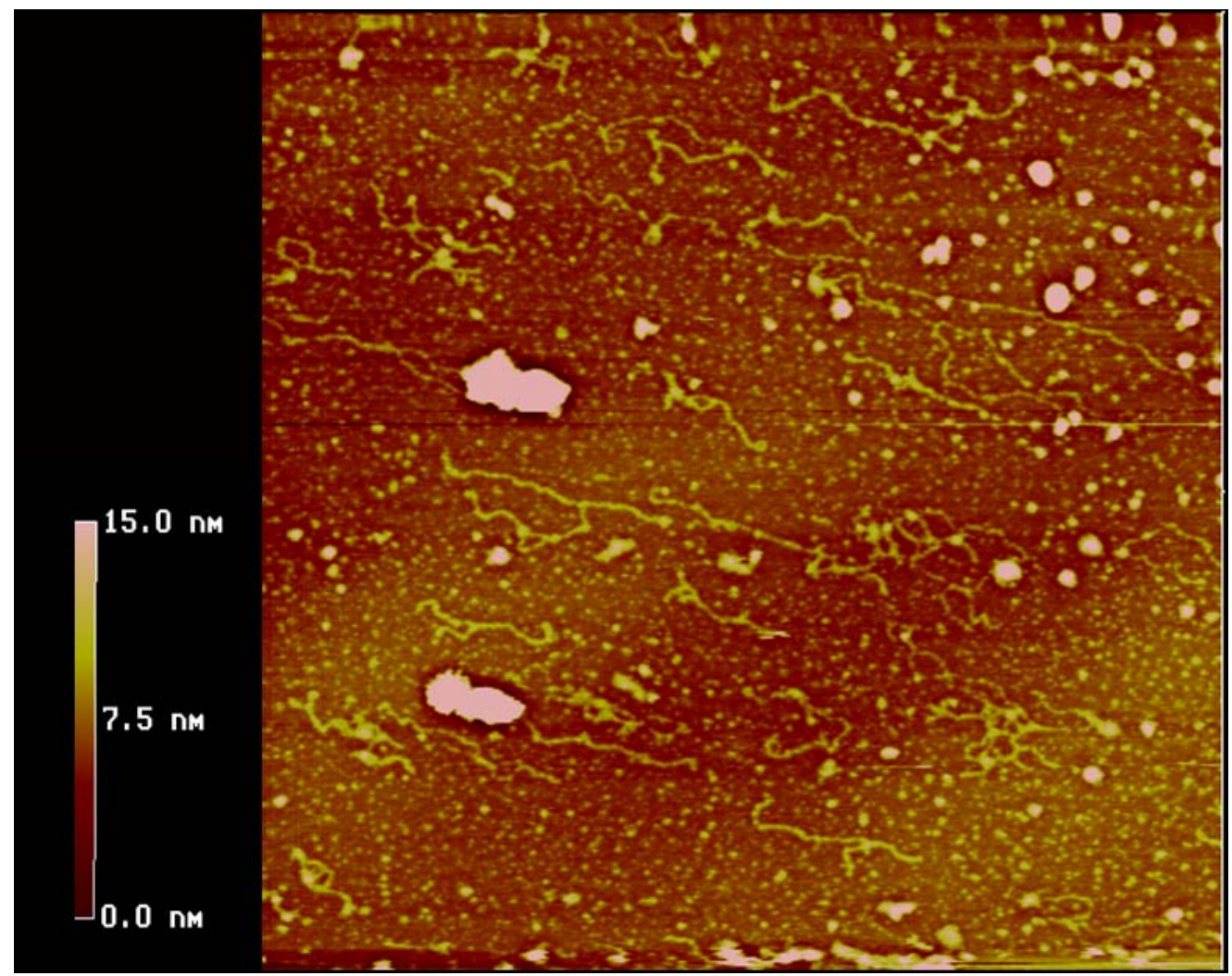

Figure 4.14: $5 \mu \mathrm{m}$ AFM scan of a sample of pPIC9 plasmid. This plasmid has 8023 base pairs, and a molecular weight of $5.2 \times 10^{6}$ Daltons. To prepare this sample, we treated a freshly cleaved mica substrate overnight with $33 \mathrm{mM} \mathrm{Mg}(\mathrm{OAc})_{2}$, sonicated it in distilled water for 20 minutes, dried it with compressed air, glow-discharged it for 40 seconds under 100 mTorr of argon, and then inverted it onto 1 drop of $190 \mathrm{nM}(990 \mathrm{ug} / \mathrm{mL})$ plasmid sample in $10 \mathrm{mM}$ Tris-HCl buffer, $\mathrm{pH} 8$ containing $1 \mathrm{mM}$ EDTA. We rinsed the sample with distilled water, and then dried it with compressed air. We imaged the sample in contact mode with a Digital Instruments Bioscope under n-propanol with a silicon nitride $\operatorname{tip}(\mathrm{k}=0.32 \mathrm{~N} / \mathrm{m})$. 
All three of these DNA images are comparable in quality to the published results of other groups.

Drying a DNA sample is not generally considered to be good for preserving its natural state. Dehydrated DNA adopts an RNA-like "A" helix, instead of its normal "B" form. This changes the number of bases per helix turn, and the resulting stress could interfere with the integrity of larger structures such as DNA lattices, which is why we had initially preferred to use the nickel-cation-mediated deposition. Unfortunately, the presence of a comparable amount of nickel in the lattice buffer precipitated the lattice DNA, so we used an isopropanol overlay on a dried sample, instead. Imaging a the same lattice sample before and after modification was not possible, because of the effect of having a drop of alcohol sitting on top of the sample during the first stage of imaging.

After preparing the lattices, we deposited them on a mica substrate for imaging. ${ }^{11}$ The manner of deposition was key. In our initial failed attempts to replicate Prof. Seeman's experiments, we had used the procedure from Hansma, et.al. cited by Erik Winfree in the original lattice paper.[2][73] Unfortunately, the procedures for imaging plasmids and lattices was actually not the same. The lattices are a substantially more delicate sample than plasmids, which are held together covalently, in a long double helix with plenty of stacking forces, and by thousands of hydrogen bonds. Lattices, on the other hand, are assembled from many 6-base DNA segments with only 12 to 18 hydrogen bonds, and the stacking associated with the six bases. The fragility of the lattices is such that, until we

\footnotetext{
${ }^{11}$ To deposit the solution containing lattices, we placed a very freshly cleaved mica substrate on the bench surrounded by a few small puddles of water. We put $4 \mu \mathrm{l}$ of lattice solution on the substrate, and inverted a petri dish over the substrate to contain the humid atmosphere from the water puddles. After three minutes, we ver gently dropped 5-6 drops of distilled water onto the substrate to rinse it, and then dried it under a very mild stream of compressed air. The dry sample lasted a long time (at least months).
} 

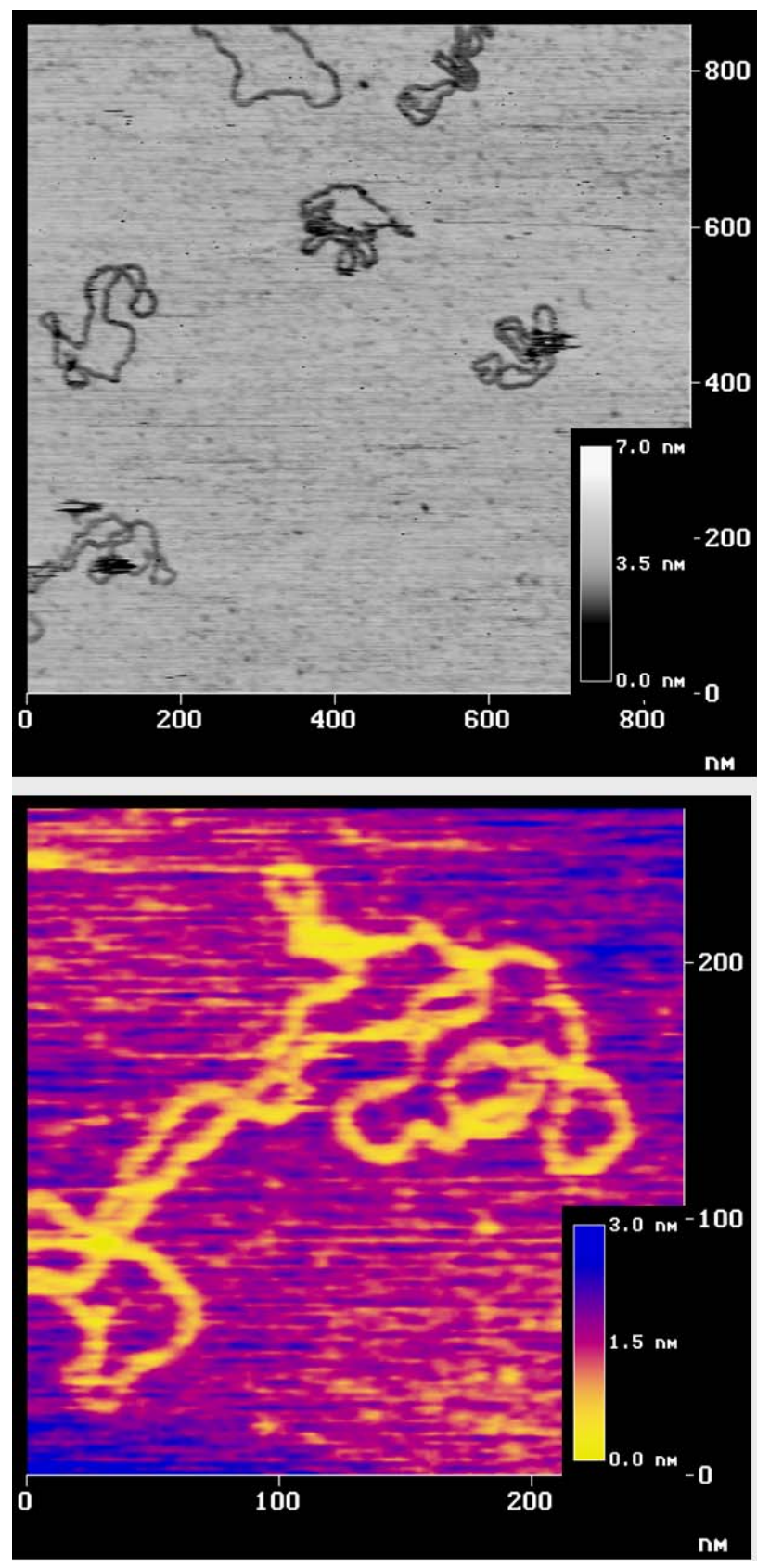

Figure 4.15: Two images of pUC19 plasmid (3500 bp, MW 2.28 $\left.\times 10^{6}\right)$. Both were collected on a Digital Instruments Multimode IIIa AFM in tapping mode. We deposited the sample on freshly cleaved mica at the same time we imaged in a $1 \frac{\mu g}{m l}$ solution of $10 \mathrm{mM}$ HEPES buffer with $5 \mathrm{mM} \mathrm{NiCl}_{2}$. The AFM tip was made of silicon nitride, with a spring constant of $0.32 \mathrm{~N} / \mathrm{m}$ and radius less than $20 \mathrm{~nm}$. 
learned the correct procedure for preparing samples in New York, it is quite possible, and even probable, that we were making lattices, and then tearing them apart during the rinse and dry steps in deposition. An image of the lattices that Erik Winfree published is shown in Figure 4.16. We were able to generate similar, or even better images, such as the image in Figure 4.17. This figure shows two images collected simultaneously. One is a topographic image, and the other is a "phase" image. ${ }^{12}$ It is possible to do a section analysis of the image to discover spacing and height between features in an image. Figure 4.18 is a section analysis of the data in Figure 4.17. Figures 4.19, 4.20, 4.21, 4.22, 4.23, 4.24, 4.25, and 4.26 show more examples of AFM images we took of four-tile system lattices.

Unfortunately, quite often, and for no obvious reason, the lattices would not form. As time progressed, the failure rate qualitatively seemed to increase somewhat. That problem may have resulted from a degradation of the strands while they were in storage, or from the problems of subjecting the strands to repeated freeze-thaw cycles. Figures $4.27,4.28,4.29$, and 4.30 show examples of what AFM of failed lattice samples looked like during imaging. Because the strands had not annealed, there was a great deal more DNA in random deposition-controlled layers on the surface. The significant failure rate for making lattices was a source of frustration because making lattices was only the first step in any procedure that would modify the lattice surface with nanocrystals. When that step failed, the experiment could not proceed. Finding a solution was essential, but we never discovered the cause of the problem before the project ended. Evidence of the extent of

\footnotetext{
${ }^{12}$ A phase image records the phase lag of the AFM tip cantilever's oscillation relative to the phase of the oscillation drive sent to the piezocrystal. It is not possible to directly extract physical meaning from this kind of image, because of its complex relationship to the properties of the sample and tip, and the scan speed. However, it often reveals qualitative features not as distinct in a topographic image, and a good-quality phase image is often an indication of a very good-quality topographic image.
} 


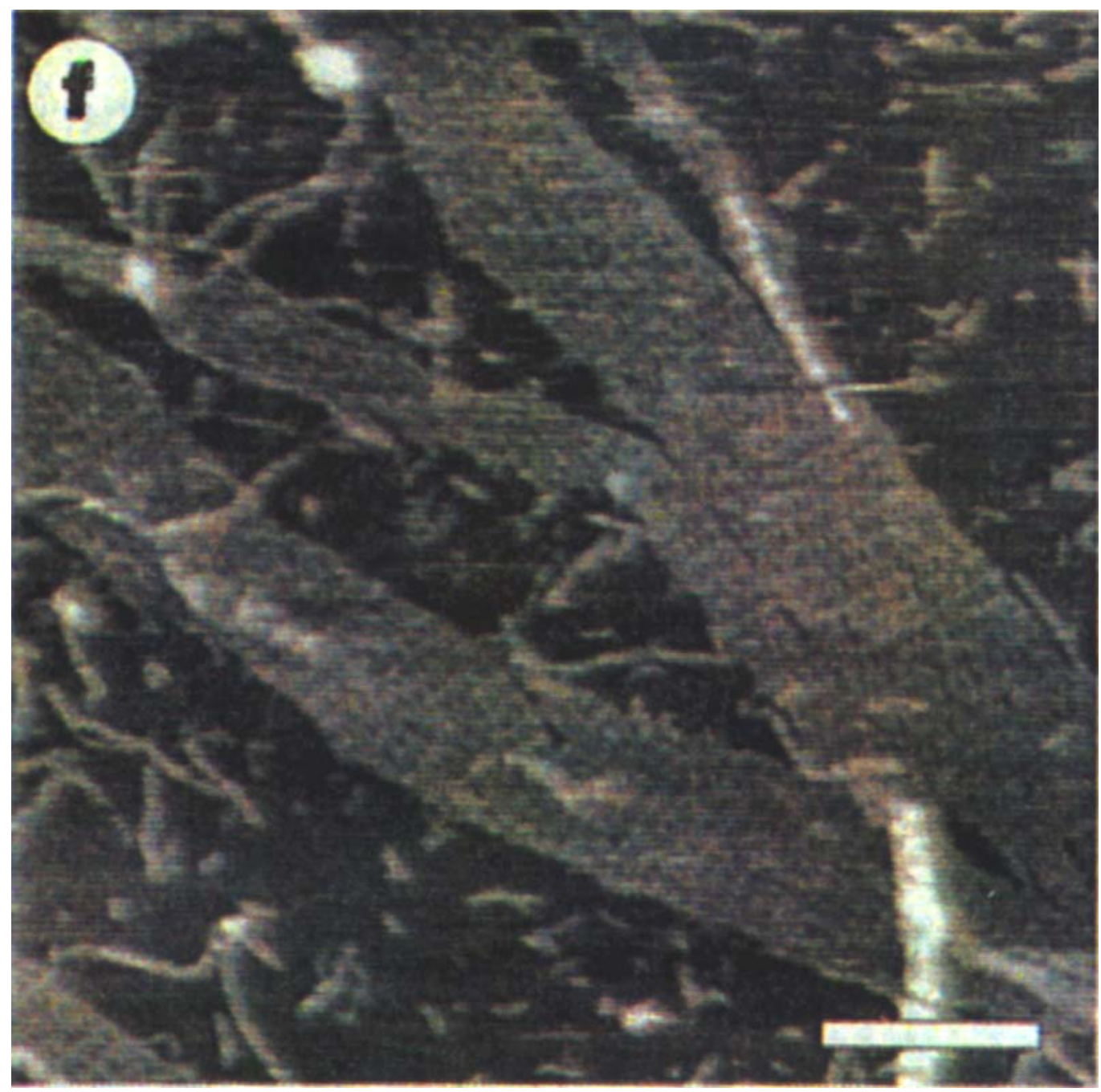

Figure 4.16: An AFM image (published by Winfree, et. al. in 1998) of lattices made of a two tile, 10 strand, system. The scale bar is $300 \mathrm{~nm}$, and the lattice has stripes created by hairpins every $32 \mathrm{~nm} .[2]$ 


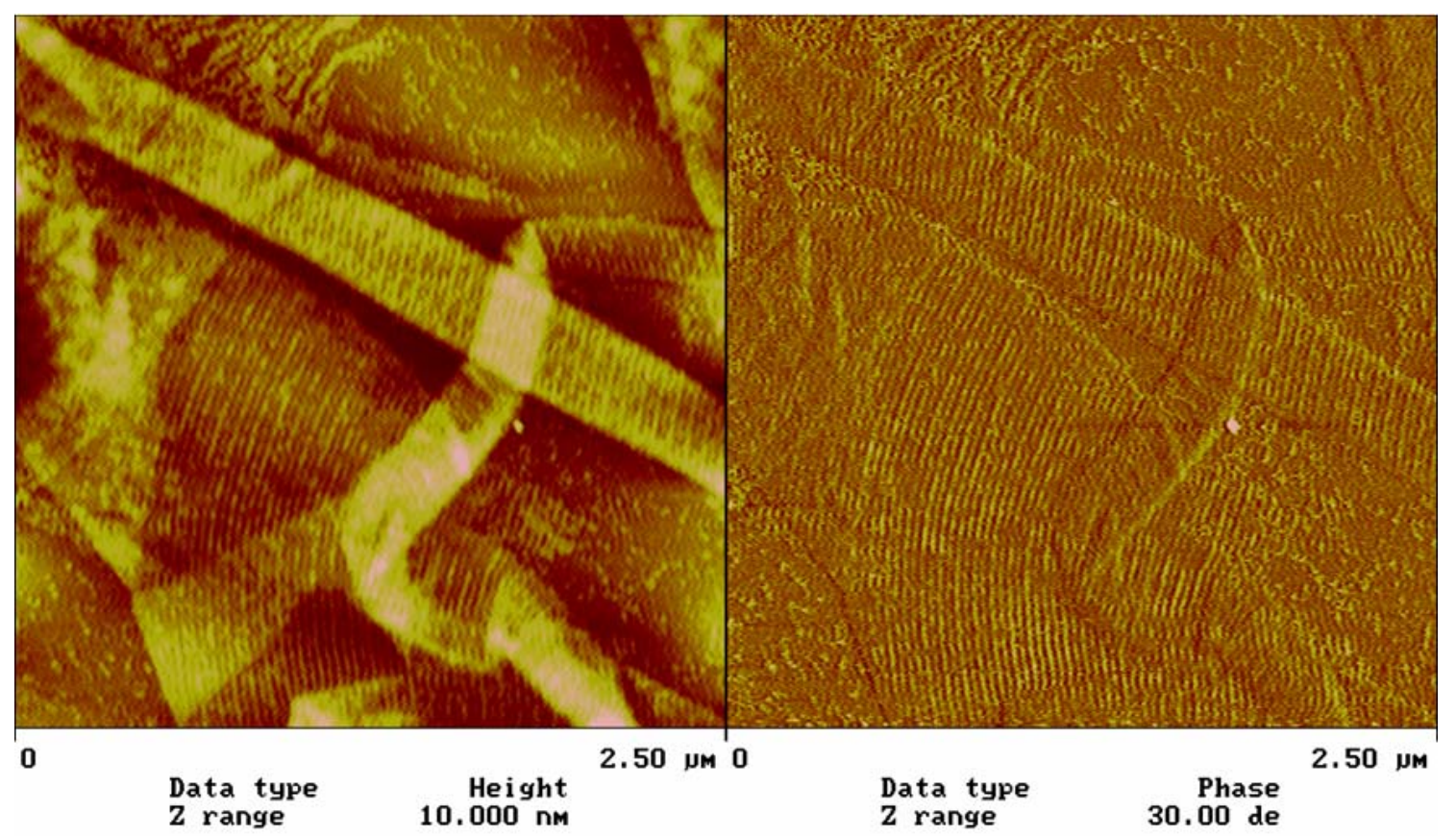

Figure 4.17: Good-quality image of four-tile system lattices with a hairpin every $64 \mathrm{~nm}$. The image on the left is a topographic image. The image on the right is a phase image. The lattices have grown quite large, and are folded back on themselves in places. 


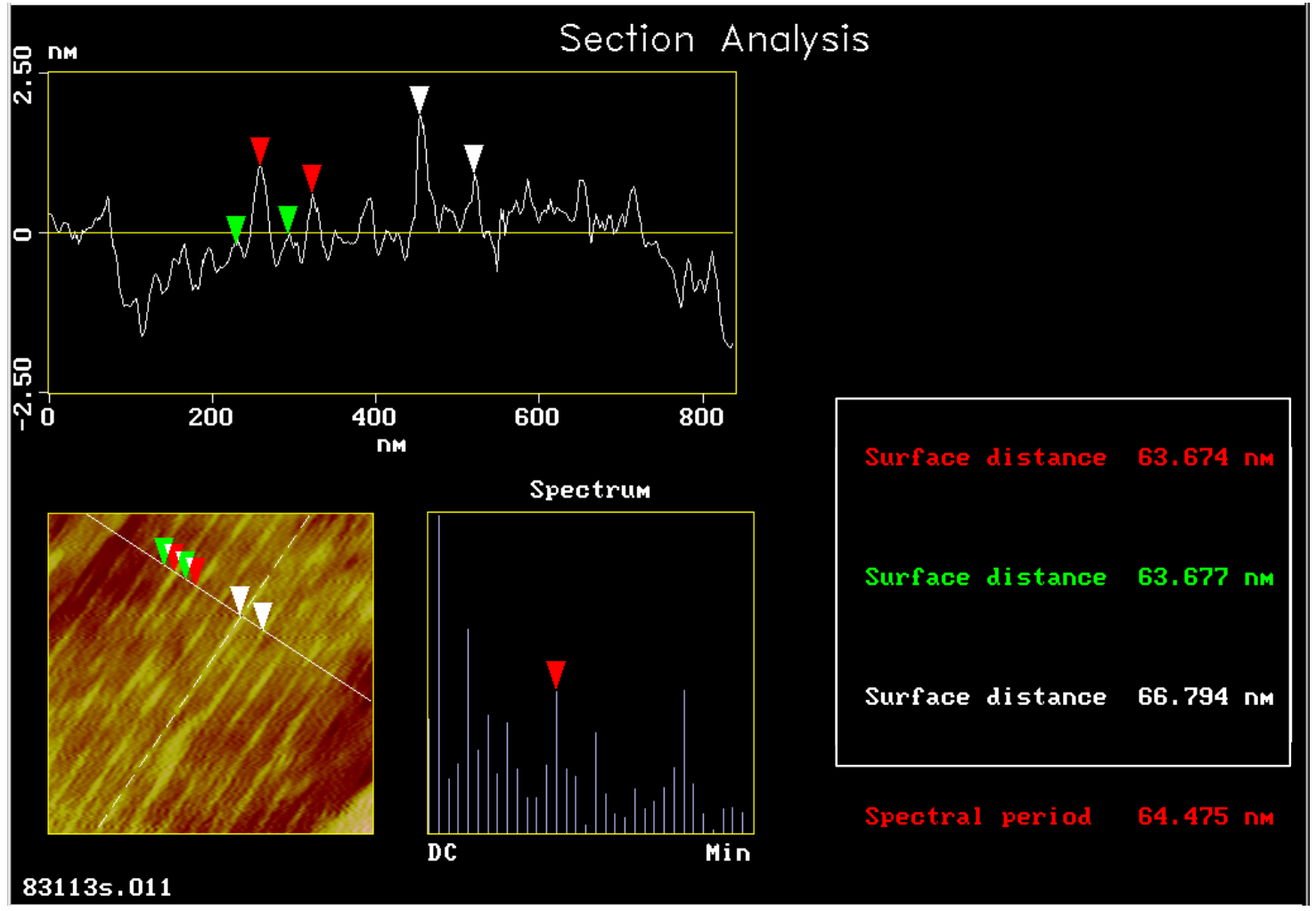

Figure 4.18: Image of a section of lattices from Figure 4.17 used to determine the periodic spacing of the ridges. The ridges were designed to be $64 \mathrm{~nm}$ apart. The measured result of $63.7 \mathrm{~nm}$ is very reasonably close to this value. 


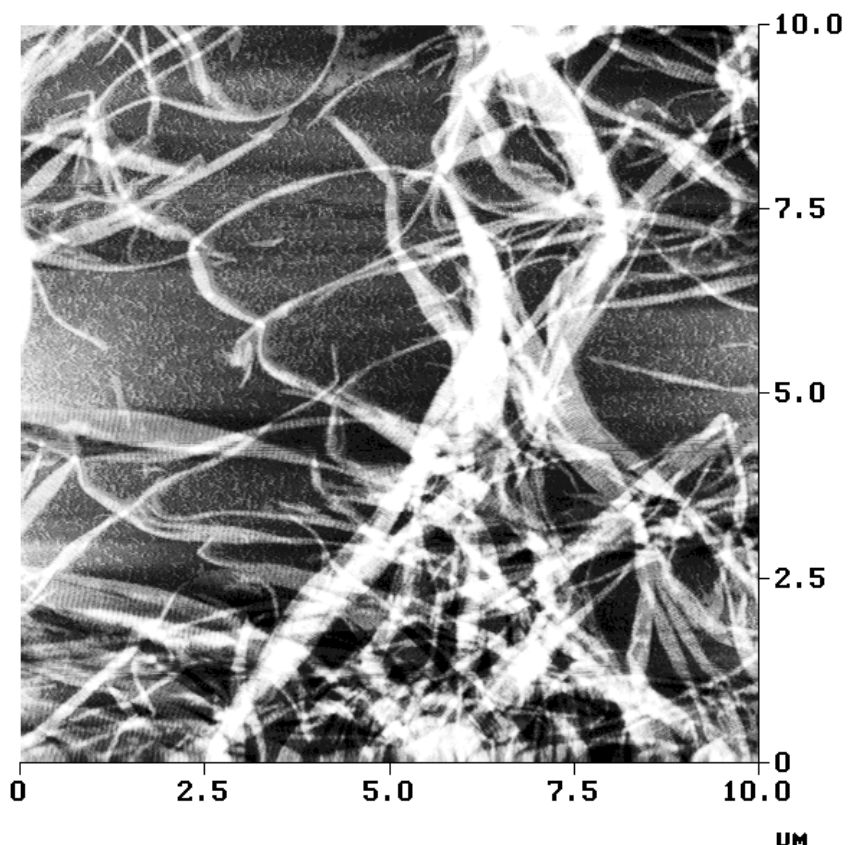

10.0

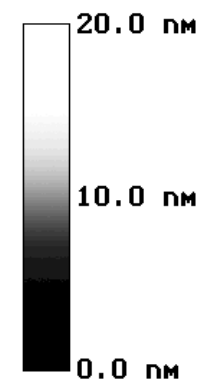

Digital Instruments NanoScope

Scan size

Scan rate

Number of samples

Image Data

$10.00 \mathrm{JM}$

Data scale

Height

$20.00 \mathrm{~nm}$

JM
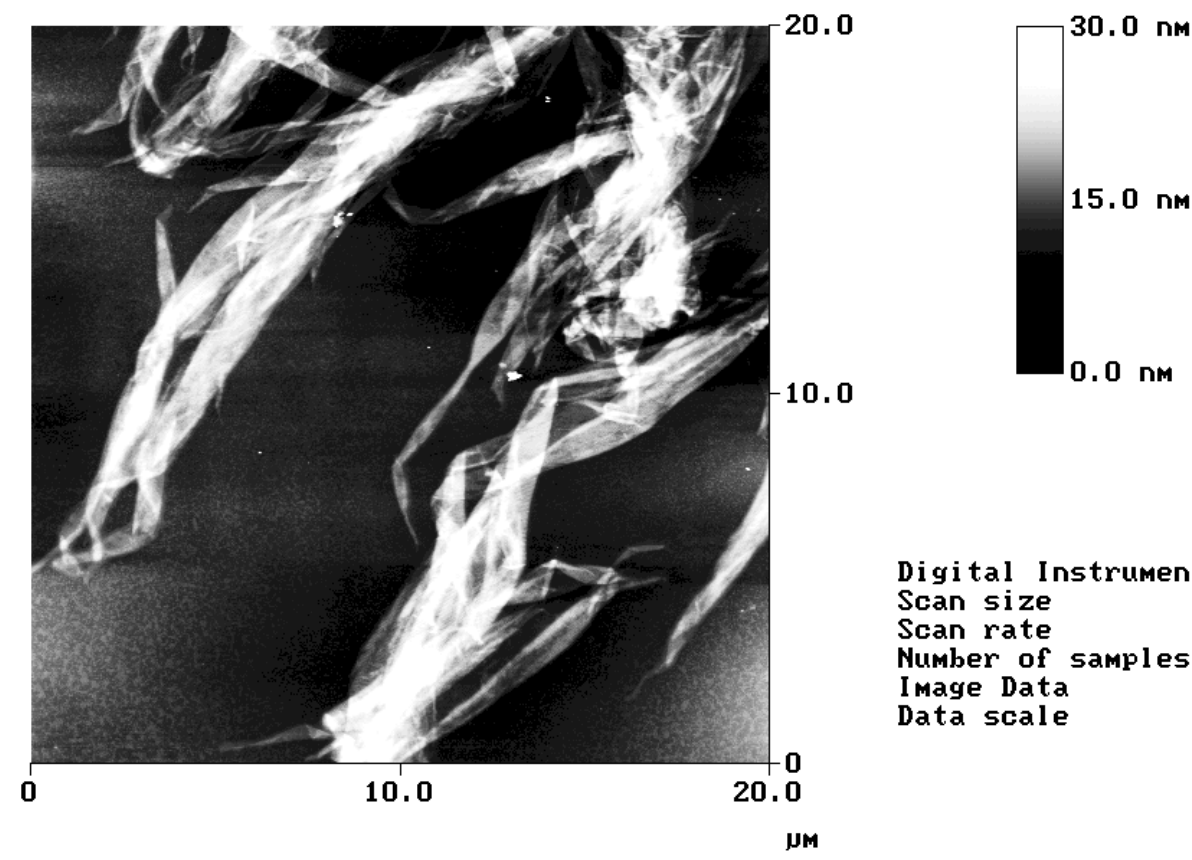

Digital Instruments NanoScope Scan size

Scan rate Number of samples

Image Data

$20.00 \mathrm{JM}$

0.8719

Data scale

Height $30.00 \mathrm{~nm}$

Figure 4.19: A $10 \mu \mathrm{m}$ AFM scan and a $20 \mu \mathrm{m}$ AFM scan of four tile DNA lattices. 


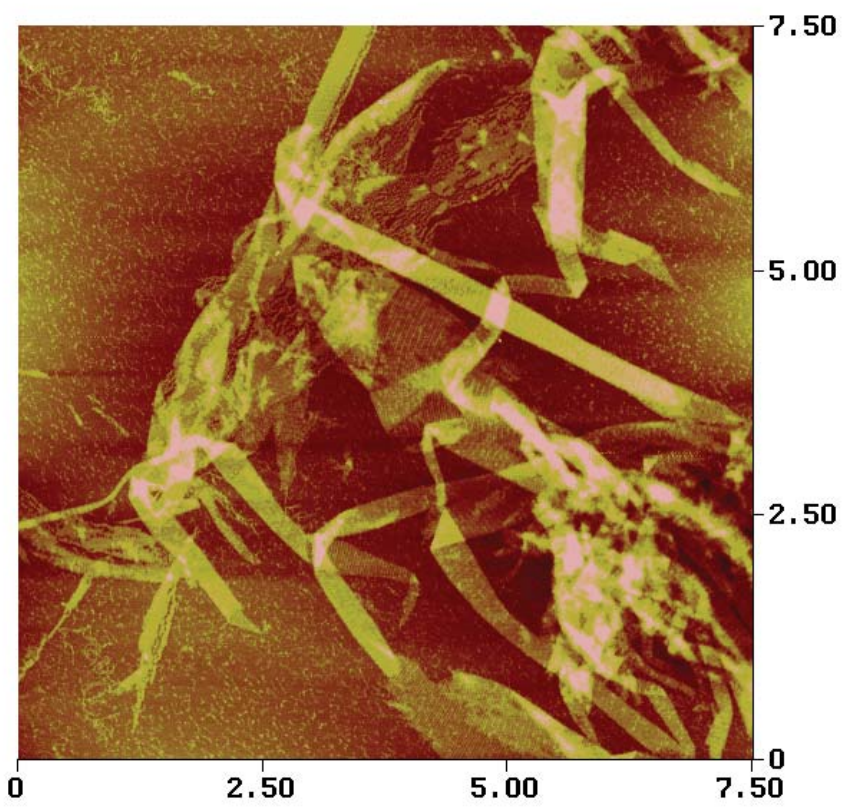

7.50

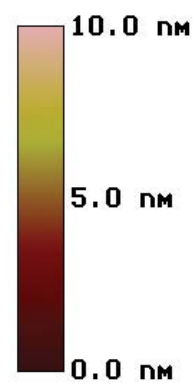

Digital Instruments NanoScope Scan size $\quad 7.515 \mathrm{\mu m}$

Scan rate Number of samples

Image Data 1. $001 \mathrm{~Hz}$

Data scale

He ight 10.00 nm

JM
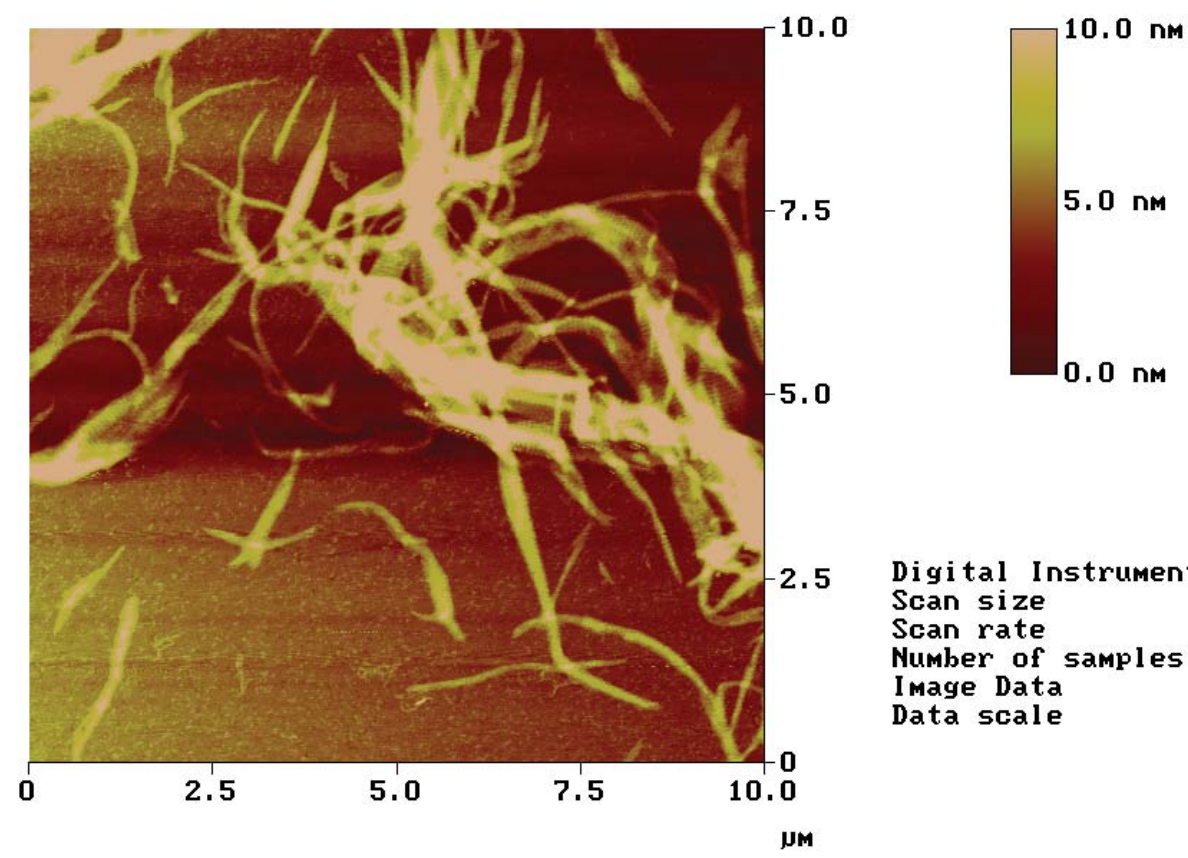

Digital Instruments NanoScope Scan size

Scan rate

Number of samples

Image Data

$10.00 \mathrm{JM}$

Data scale

Height

$10.00 \mathrm{~nm}$

Figure 4.20: A $7.5 \mu \mathrm{m}$ AFM scan and a $10 \mu \mathrm{m}$ AFM scan of four tile DNA lattices. 


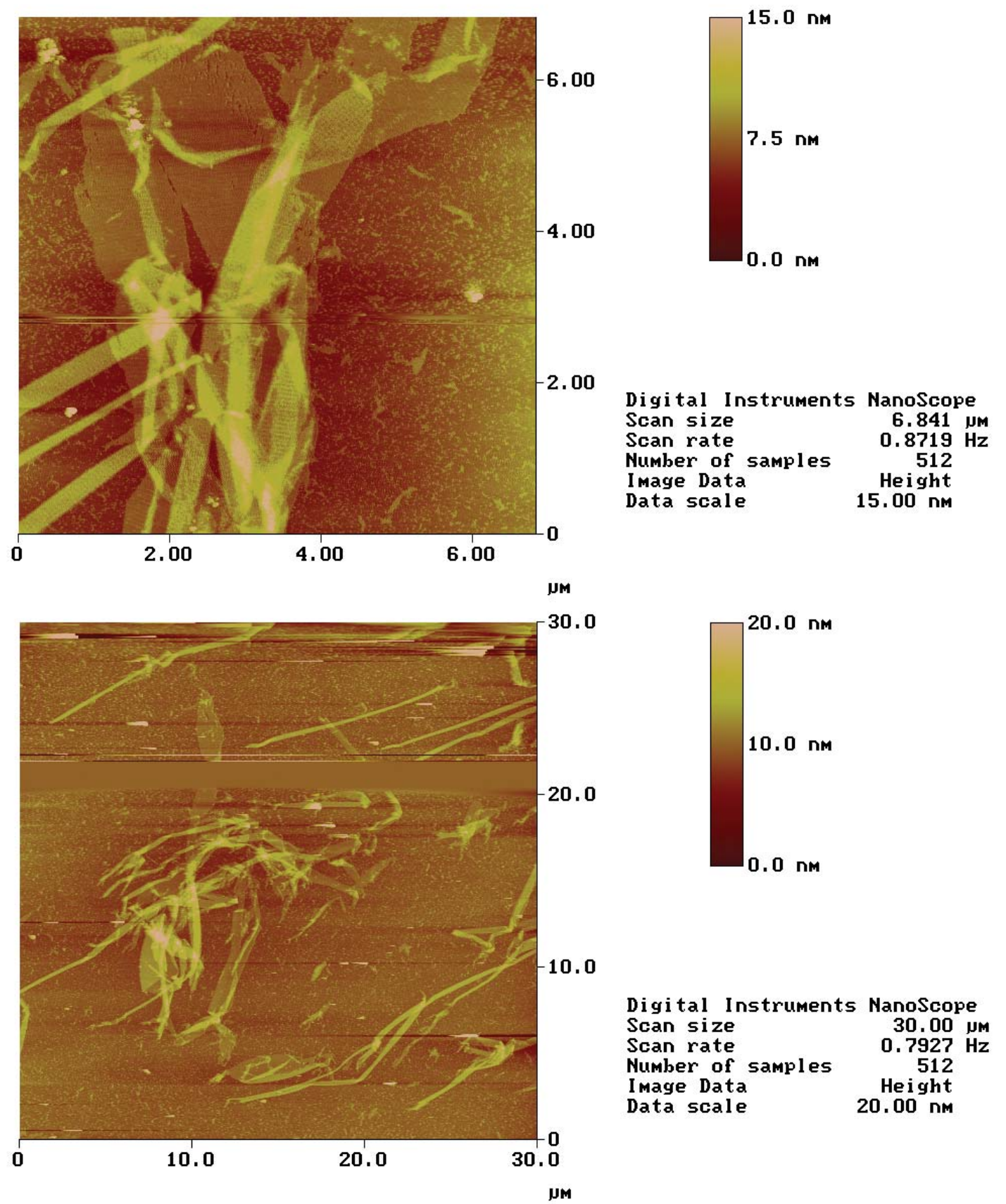

Figure 4.21: A $6.8 \mu \mathrm{m}$ AFM scan and a $30 \mu \mathrm{m}$ AFM scan of four tile DNA lattices. For part of the $30 \mu \mathrm{m}$ scan the probe temporarily lost contact with the sample, but at the necessarily slow $0.79 \mathrm{~Hz}$ scan rate, that problem is not surprising, and was hard to prevent. Our capacity to do a $30 \mu \mathrm{m}$ scan of this kind of sample was actually quite good. 

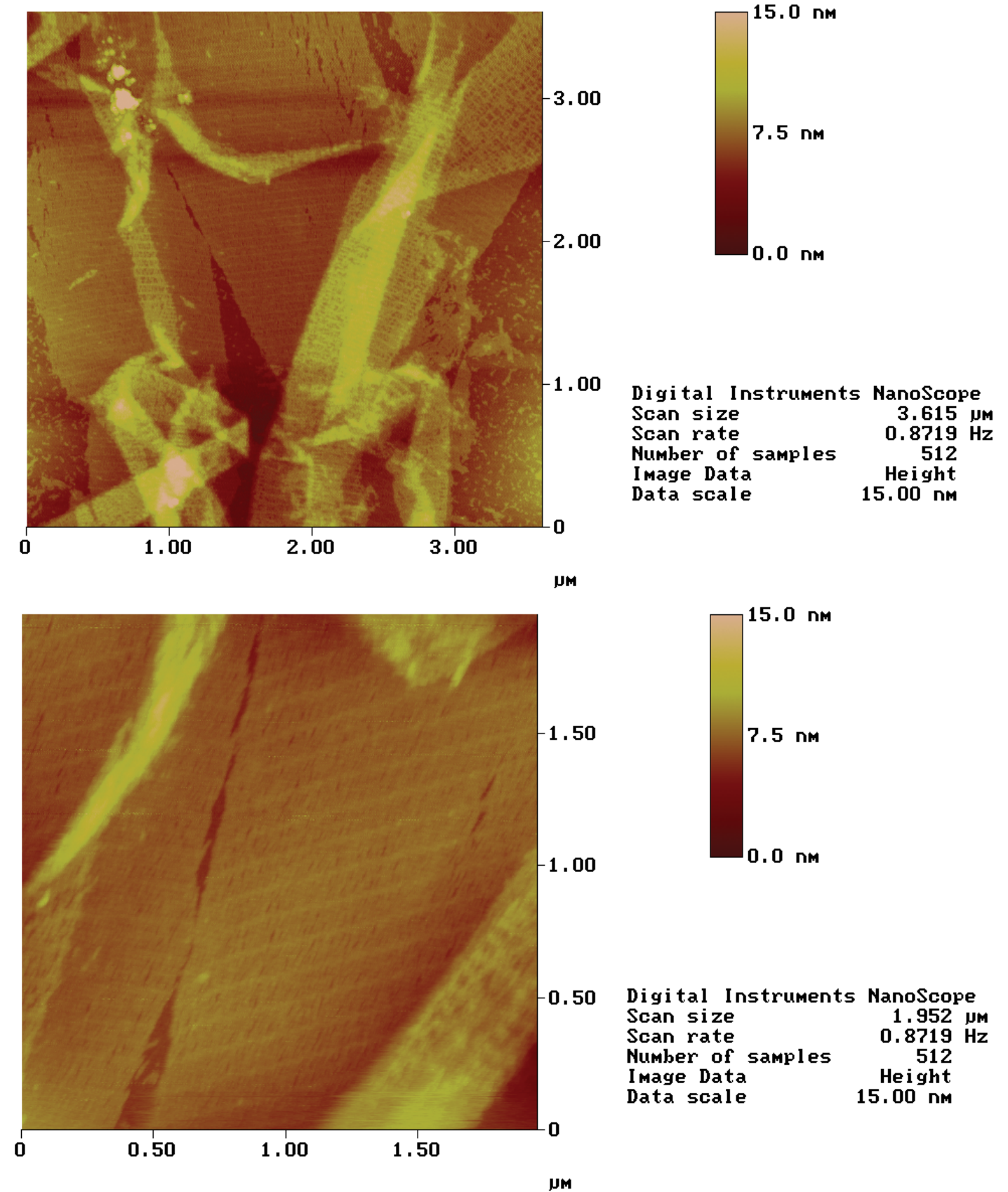

Figure 4.22: A $3.6 \mu \mathrm{m}$ and a $2.0 \mu \mathrm{m}$ AFM scan of two lattice samples. In the lower image, the rows of DNA hairpins on Tile D are clearly visible, along with the available sites for hairpin or gold-conjugate addition on Tile B, which make a fainter line in between the Tile $\mathrm{D}$ ridges. 

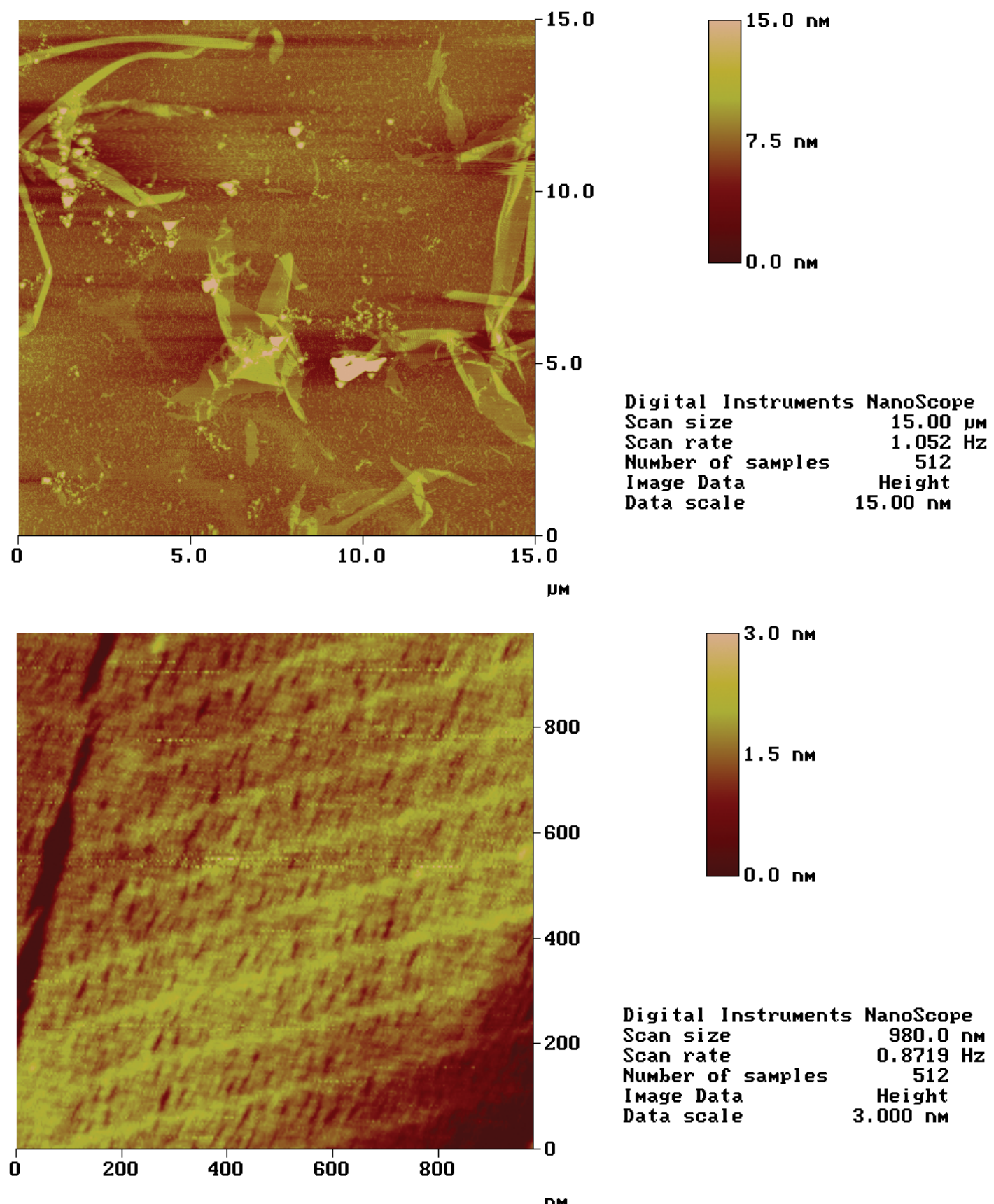

Digital Instruments NanoScope

Scan size

Scan rate

Number of samples

Image Data

$980.0 \mathrm{~nm}$

Data scale

$0.8719 \mathrm{~Hz}$

512

Height $3.000 \mathrm{~nm}$

Figure 4.23: A $15 \mu \mathrm{m}$ scan and a $980 \mathrm{~nm}$ lattice scan. It was hard to get good-quality images at a small scan size (like the one on the bottom) because the DNA seemed to be dragged around more by the AFM tip. This image was exceptionally good for its size. 

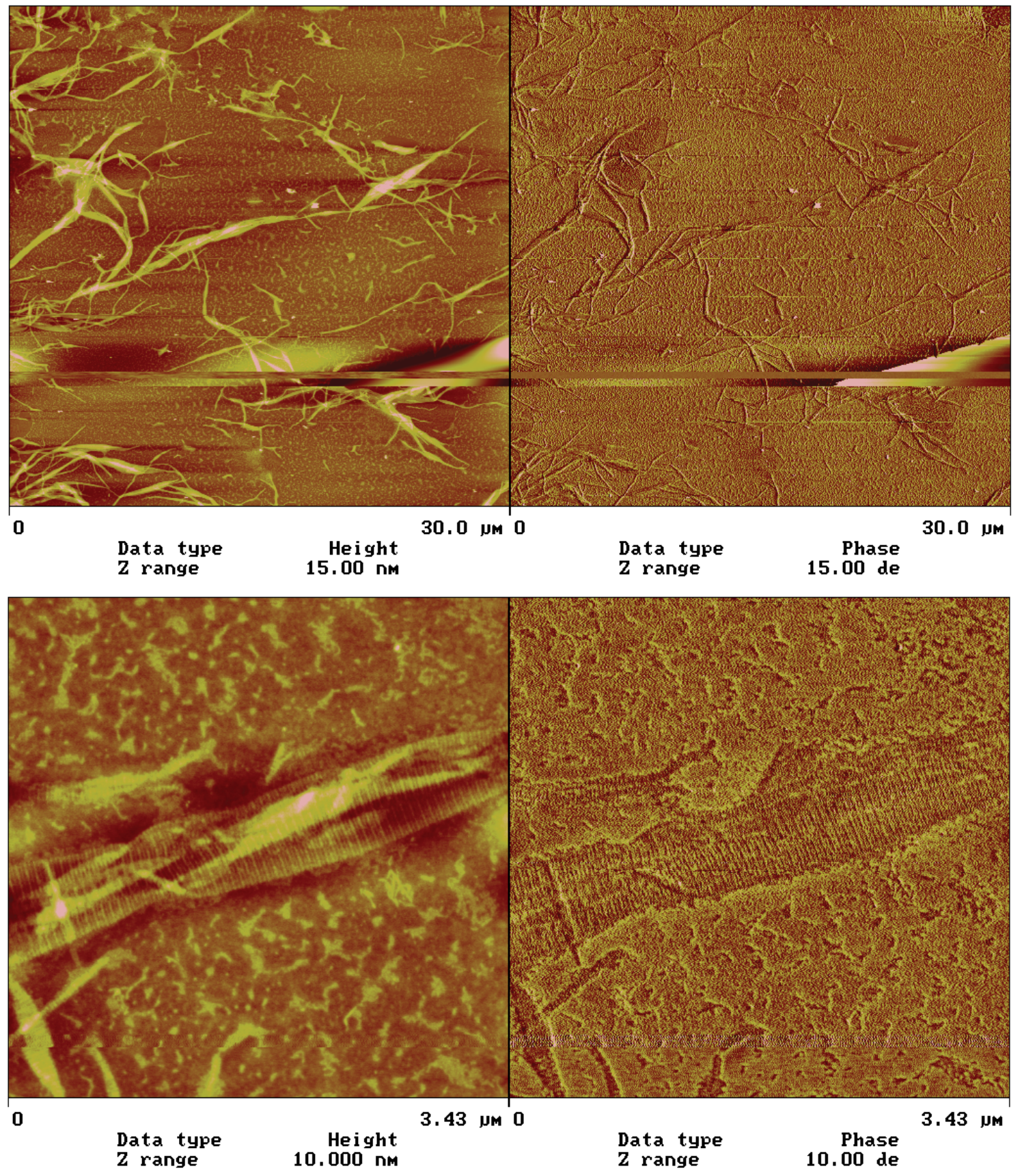

Figure 4.24: A $30 \mu \mathrm{m}$ scan and a $3.43 \mu \mathrm{m}$ scan. These images have some noise in the phase image, but the topography mode gave a nice image. The phase images on the right were more typical of a phase image on a good, but not exceptional day. Better phase images were exceptional. The top image, once again, has a region where the AFM tip lost contact with the sample, but given the large scan size, that problem was expected. 

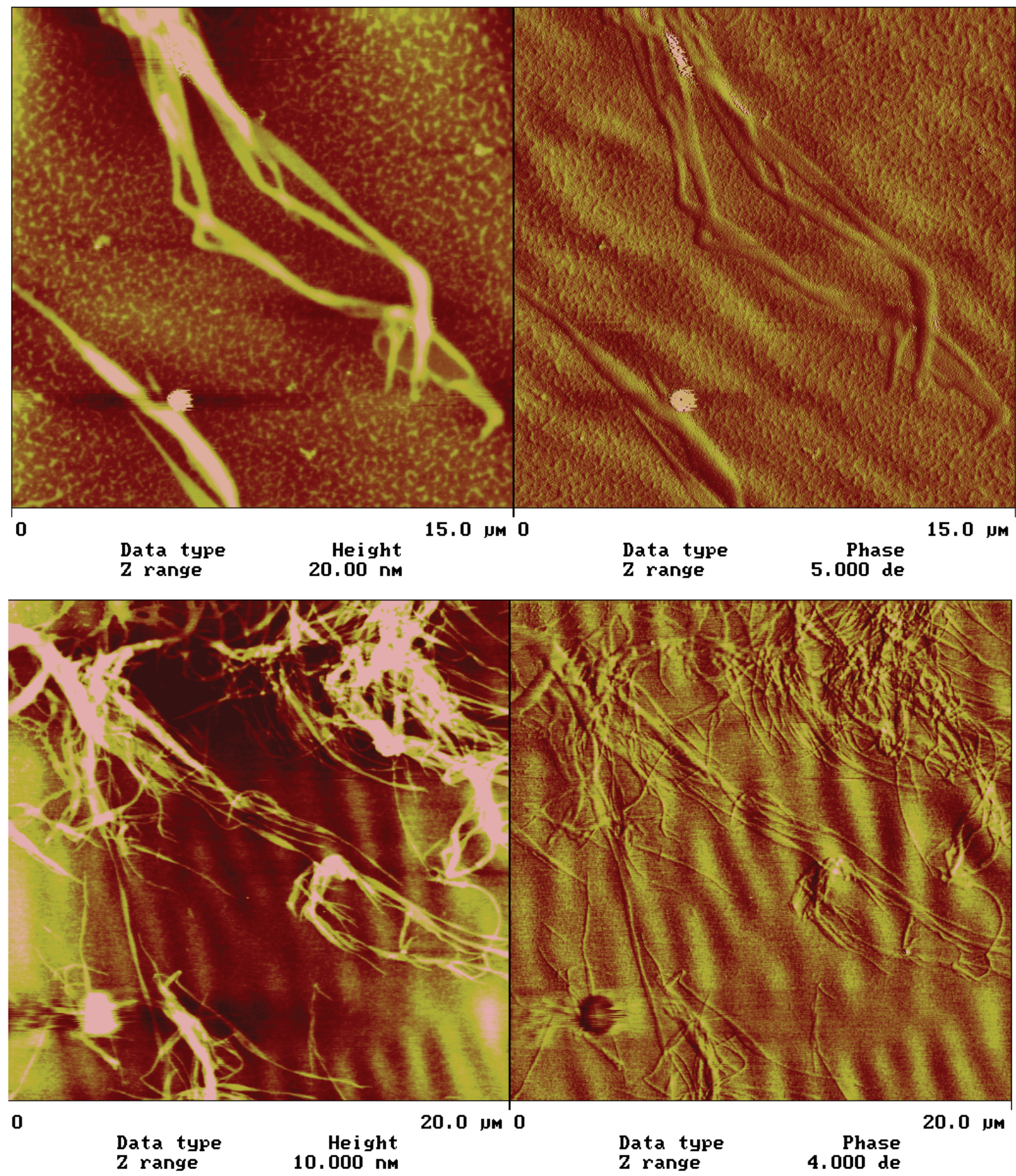

Figure 4.25: $15 \mu \mathrm{m}$ and a $20 \mu \mathrm{m}$ scans. 


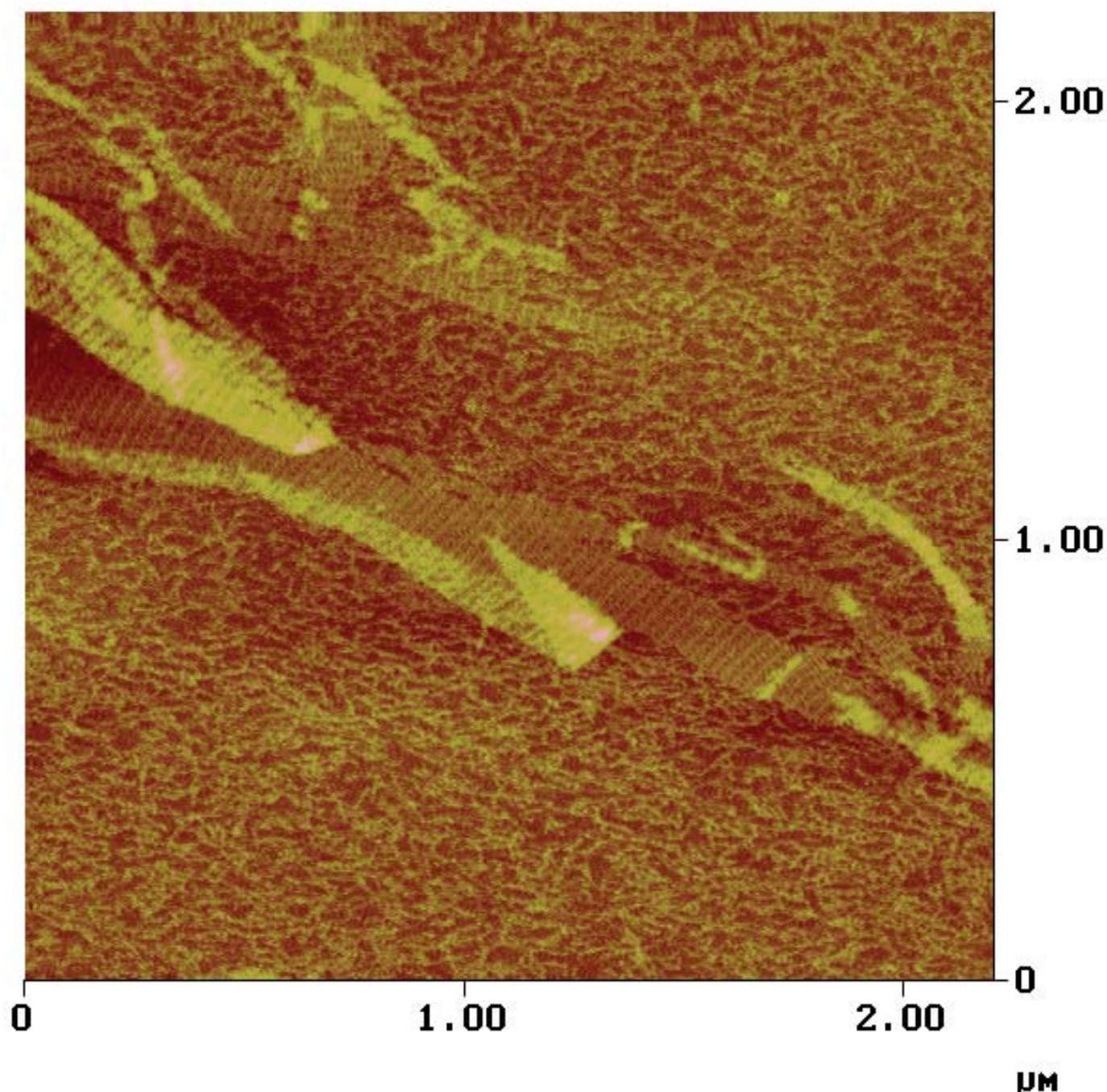

Figure 4.26: A scan showing lattices surrounded by a large amount of unincorporated DNA. This lattice reaction was not very successful, and there are many smaller structures on the substrate. 

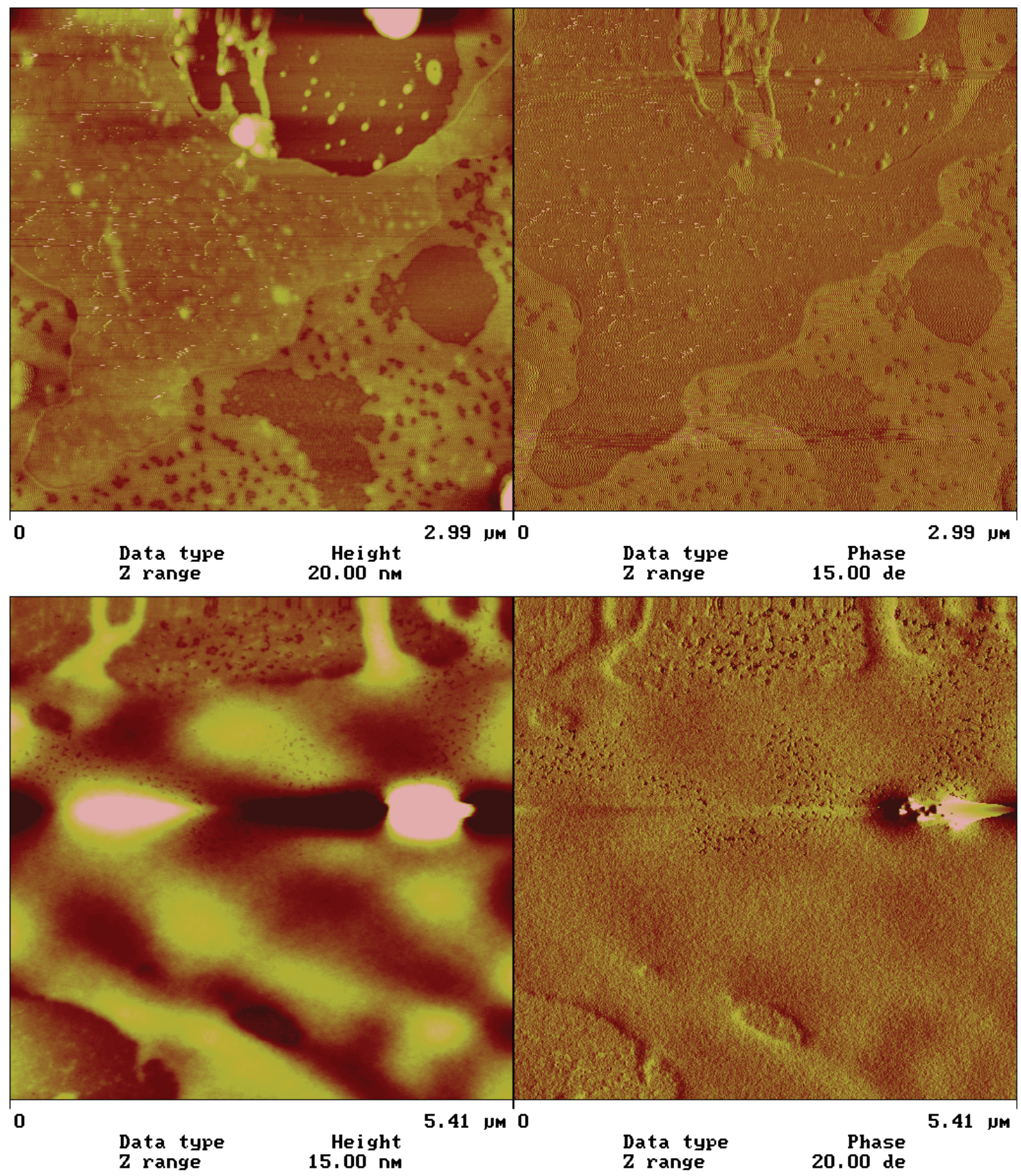

Figure 4.27: Two images of separate failed lattice batches. The DNA has deposited according to the drying pattern of the solution. 

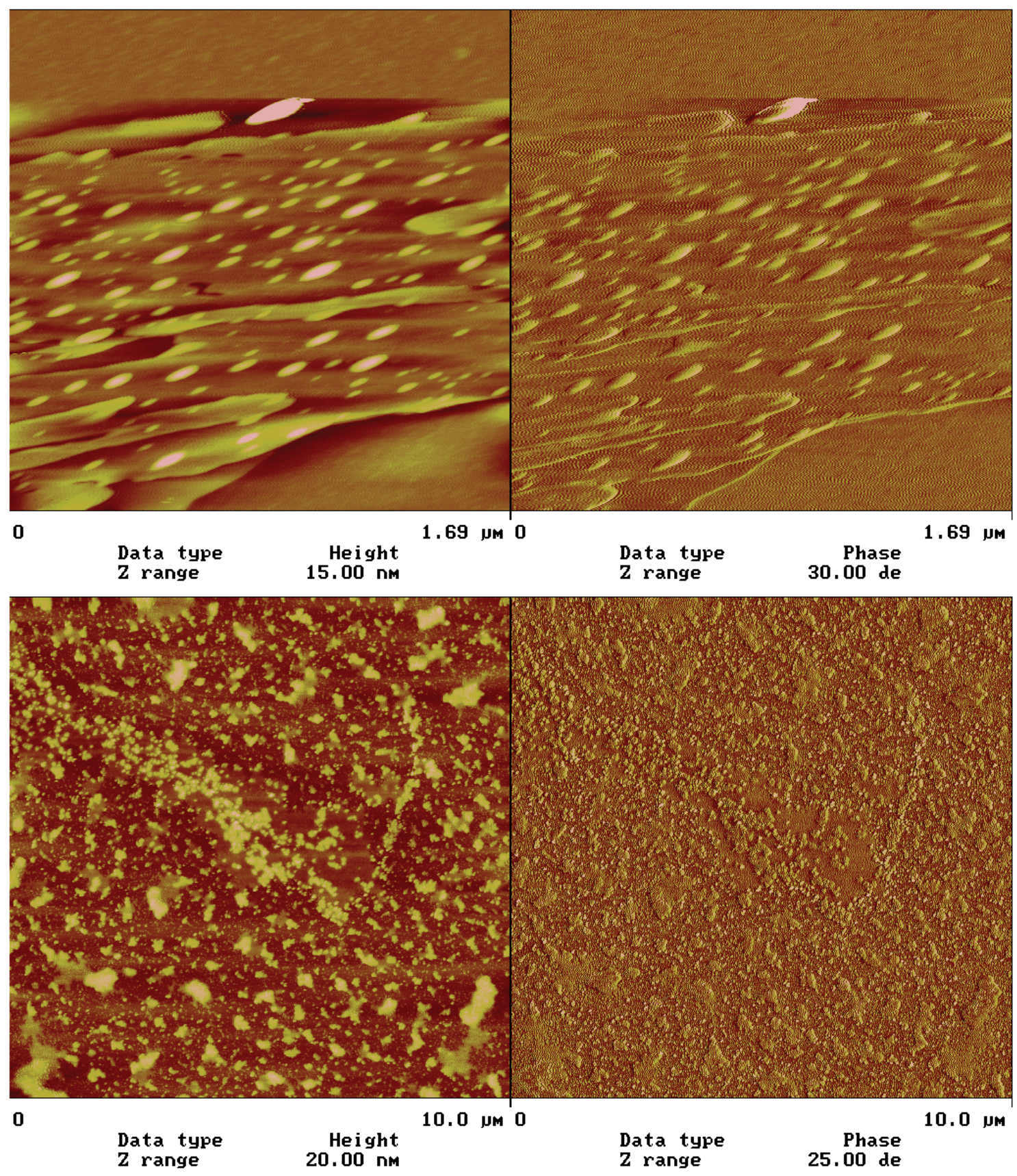

Figure 4.28: Two more images of failed lattice batches. It seems that perhaps some small structures have formed in the sample in the bottom image. 

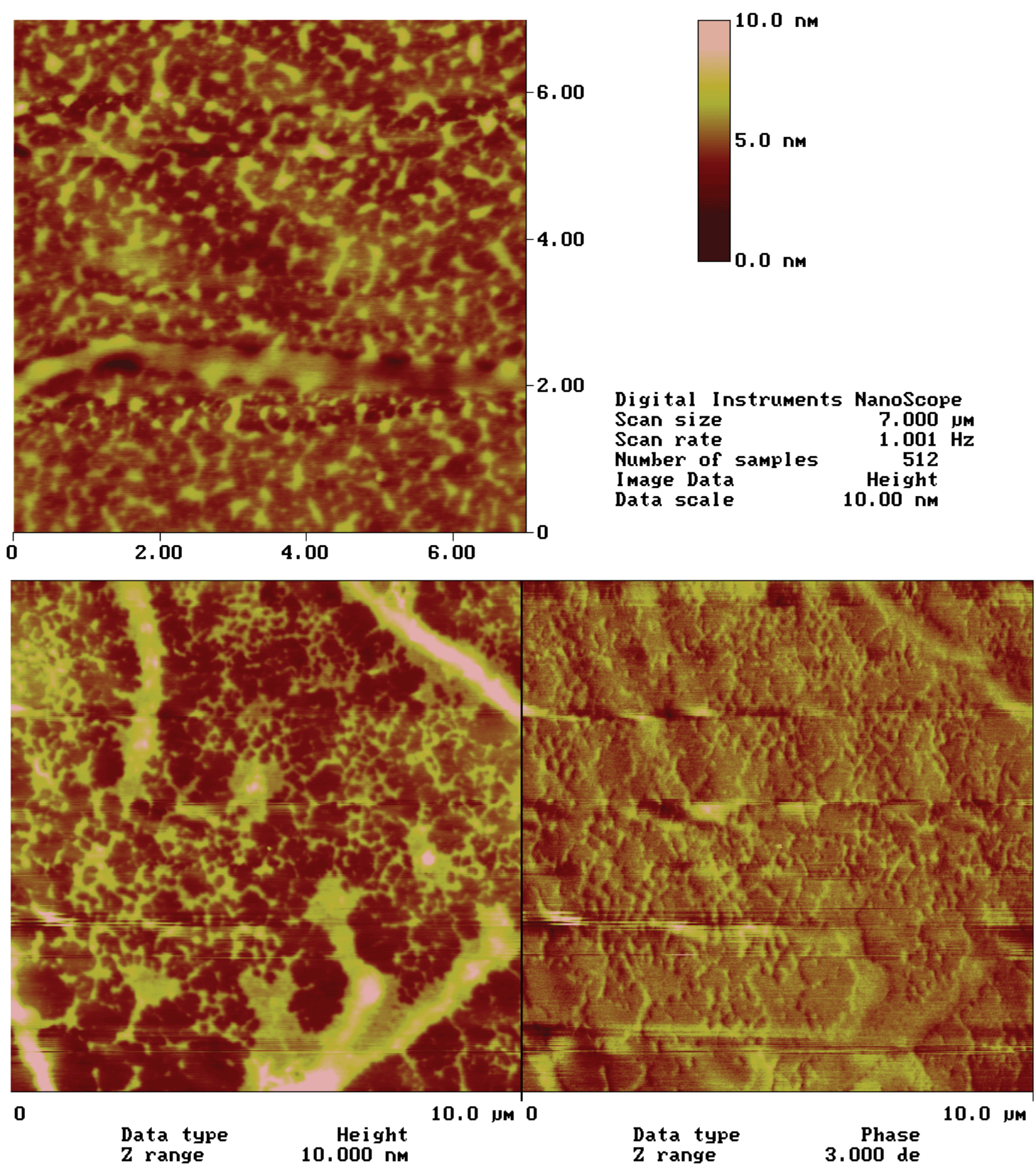

Figure 4.29: Yet two more failed lattice batches. This kind of image was common in unformed lattice batches. 

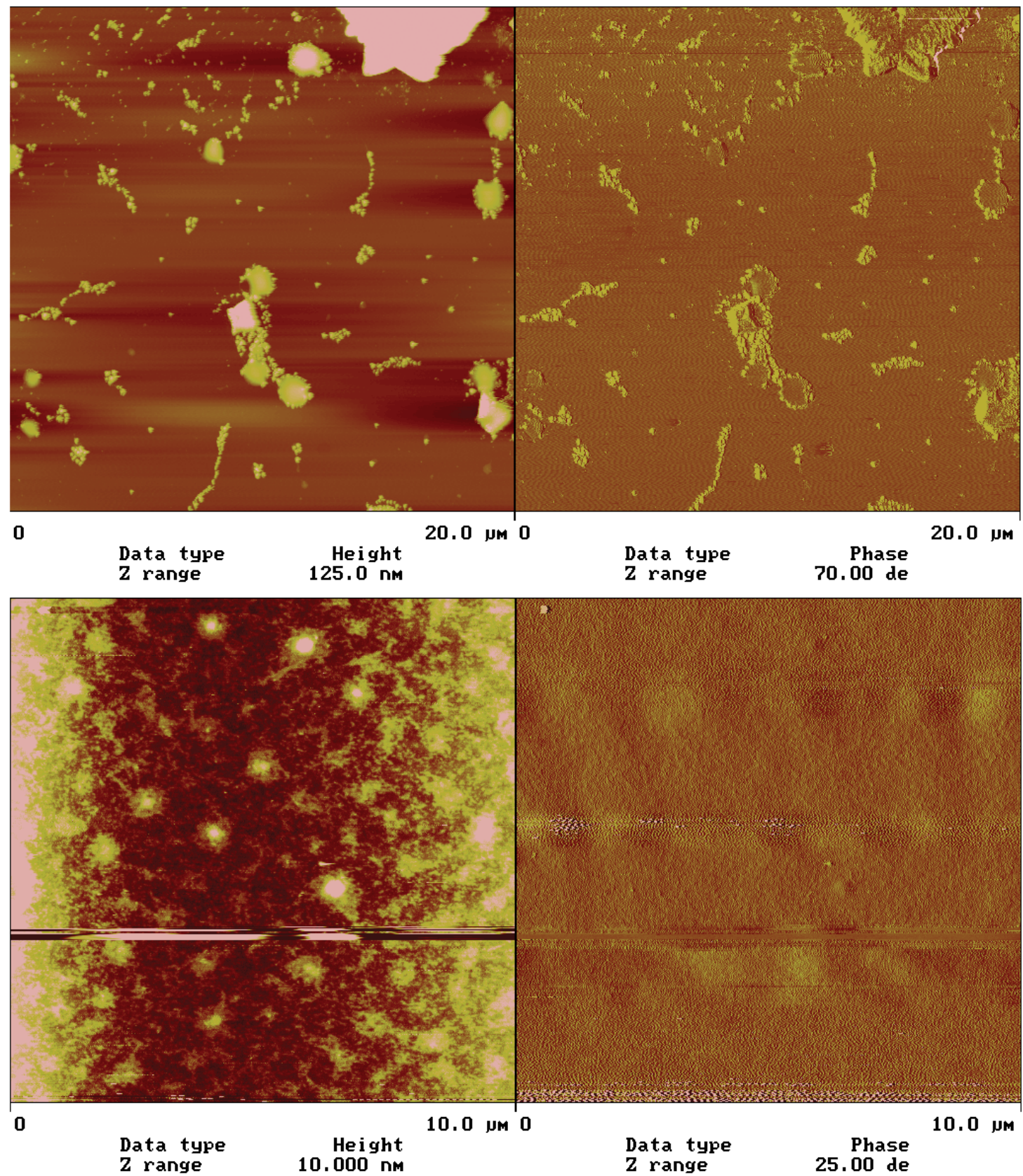

Figure 4.30: Two last failed lattice batches. DNA strands and small structures are visible in the bottom image. The bottom image can be compared to the image in Figure 4.26, where a few lattices still formed amongst a similar mess. 
the problem during most of a year is displayed in the three-dimensional plot in Figure 4.31. This figure shows that, as time passed, the chance of success in lattice imaging, especially on the first attempt, decreased. Probably, if the next batch of DNA had been completed (purification was in progress until work on this project stopped), the success rate of making lattices would have improved.

\subsection{Factors Influencing Increased Lattice Size}

When the lattices were forming well, we had better success than Prof. Seeman at growing large arrays. The arrays always grew much faster in one direction (perpendicular to the hairpin ridges) than the other. As a result the lattices we saw were quite long, but skinny. The lattices, in some cases, appeared to grow in that manner, and in other cases (such in Erik Winfree's image in Figure 4.16) seemed to tear after growing to give long, skinny fragments. The longest lattices we grew seemed to be 20 or $30 \mu \mathrm{m}$, an improvement over the 2 X $8 \mu \mathrm{m}$ reported by Winfree, et. al. Our ability to make larger structures was complemented by our ability to image them. Our AFM scanner had a much larger maximum scan size than Prof. Seeman's, so pictures like the ones here have not been published to date.

\subsection{Factors Influencing Successful Nanocrystal Patterning}

The primary factors that governed our ability to pattern nanocrystals were

1. providing binding specificity for the nanocrystals through their attached DNA 


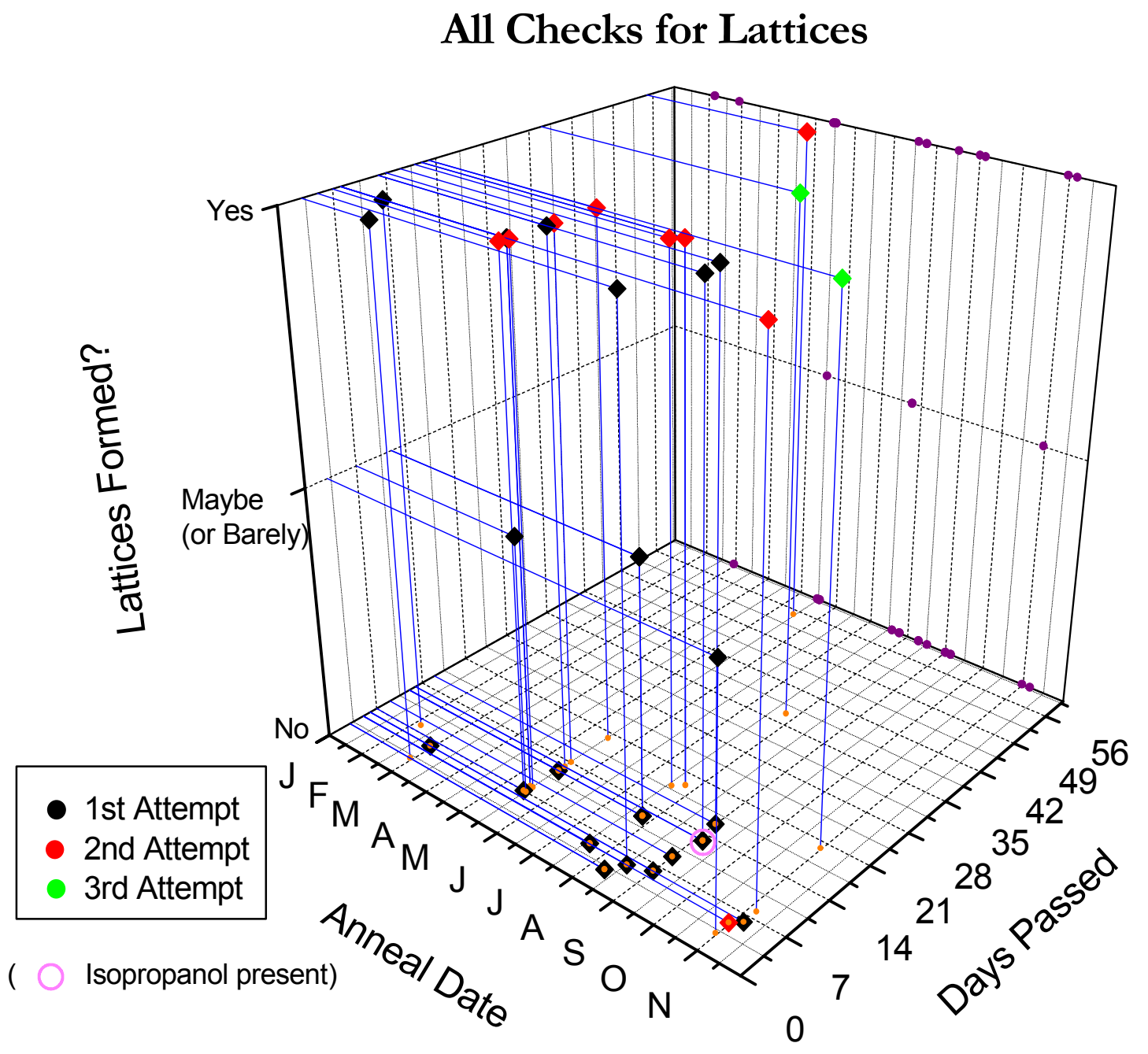

Figure 4.31: Plot showing whether lattices formed in any particular experiment versus time of year, and time left annealing. The time of year data could give clues to whether the DNA strands had degraded over time. Many experiments were conducted simultaneously, so every dot does not necessarily correspond to a single experiment. "1st Attempt," "2nd Attempt," etc. correspond to the first, second, etc. attempts to look for lattices in the sample under AFM. Sometimes lattices would not form before the initial check, but after the passage of more time, would form. 
2. preserving the lattice sample integrity throughout the treatment with nanocrystals, any purification steps, deposition, and during imaging

3. making lattices form successfully in the first place

4. controlling non-specific binding of nanocrystals

The fourth factor provided the overwhelming hurdle blocking the overall success of the project. Our attempts to overcome those problems were severely retarded by factor $\# 3$, the failure of the lattices to form on a consistent basis from the DNA samples we had purified.

\subsection{Results of Patterning Attempts}

The results of our initial attempt to pattern nanocrystals is shown in Figure 4.32. A repeat of the experiment gave similar results. The technique we designed to address that problem was the electrodialysis procedure we discussed in Section 4.5. We wanted to proceed with adding the gold particles to the lattices, without worrying about the nonspecific binding, and assume that our specific, intended binding would hold at least some of the gold particles to the lattices. We then planned to remove the non-specifically-bound nanoparticles using the electrodialysis technique. We demonstrated that the lattices could withstand the electrodialysis treatment, and that electrodialysis would even improve the quality of subsequent lattice images, as shown in Figure 4.33. Unfortunately, because of the complications in reliably making lattices, we did not end up with a gold-particlemodified lattice sample to electrodialyze before our work on the project stopped, so we 


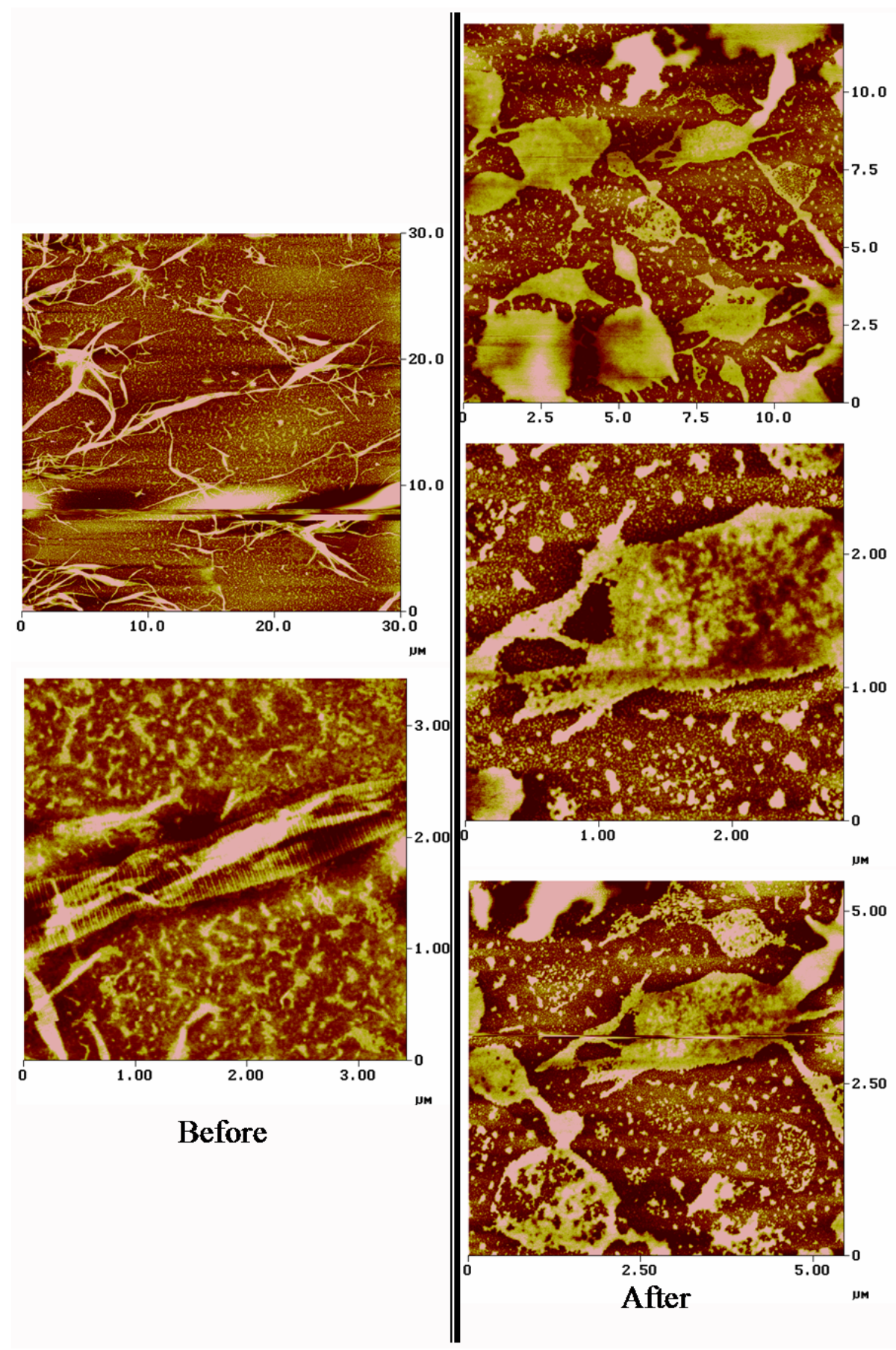

Figure 4.32: Before and after pictures for a lattice sample with $5 \mathrm{~nm}$ gold conjugates added. The gold binds non-specifically everywhere on the surface of the lattices, instead of binding specifically to the designed and available hairpin binding sites. 

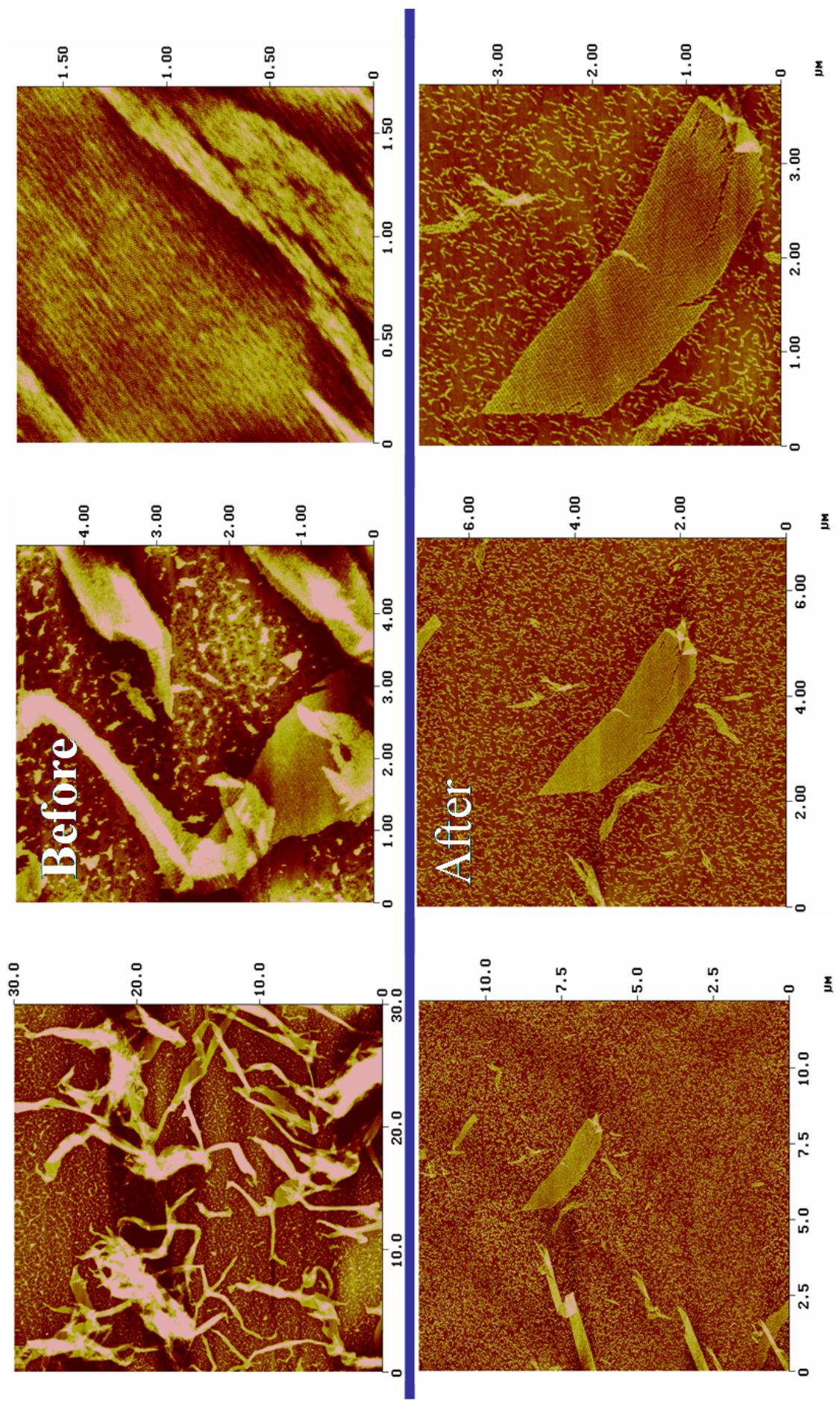

Figure 4.33: Before and after pictures of a sample of electrodialyzed lattices. 

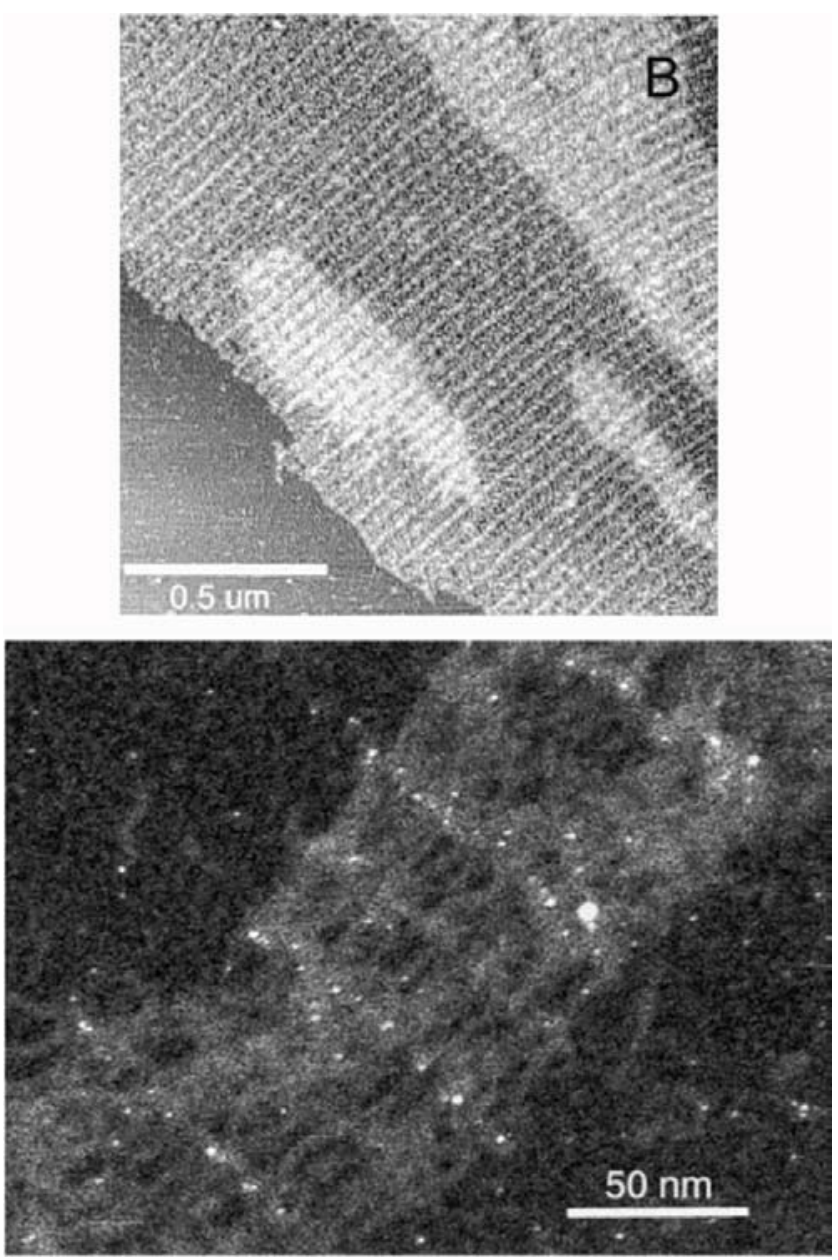

Figure 4.34: The top image, from Xiao, et.al. shows lattices before addition of gold, and the bottom shows lattices after gold addition.[17]

don't know if this technique would have removed non-specifically bound particles.

During a simultaneous period as the experiments described here, another group worked on the same idea.[17] They also had difficulties, but they published one picture that showed what could be incomplete, irregular lines of gold particles on the lattice structures. That picture is shown in Figure 4.34. 


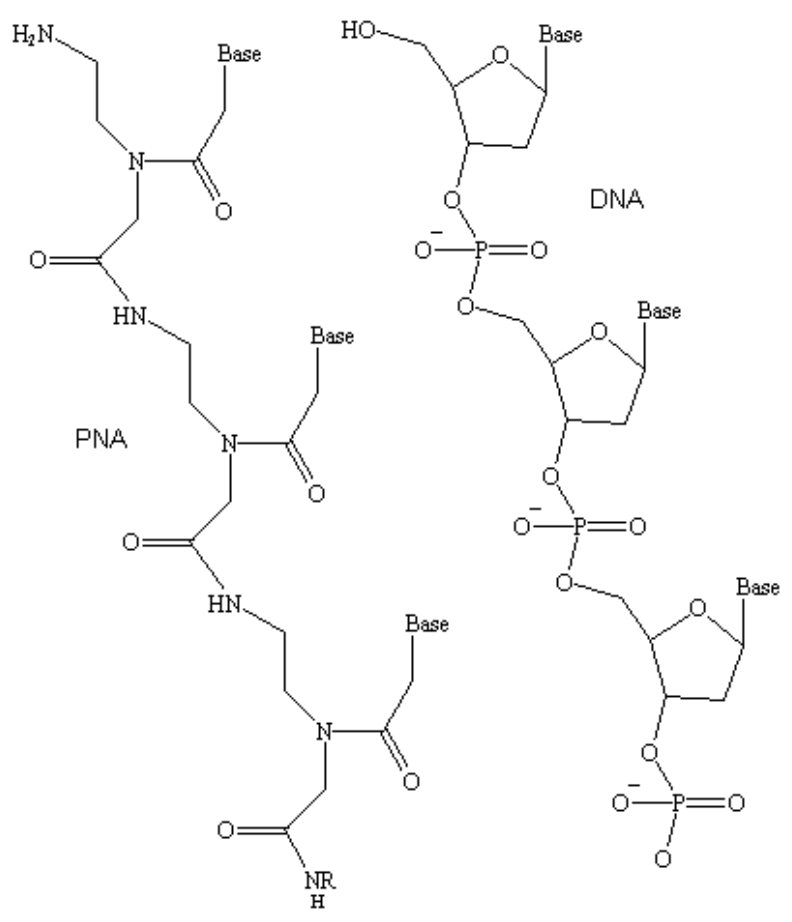

Figure 4.35: A comparison of the backbone structures of PNA and DNA. The figure shows PNA on the left, and DNA on the right.

\subsection{Future Work on Patterning Nanocrystals on Lattices}

Further work on this project at New York University has addressed the problem of the incompatibility of the gold nanocrystals with the presence of $\mathrm{Mg}^{2+}$ ions. They have redesigned the DNA for the lattice system to include "PNA" surrounding the crossover points in the tiles of the DNA lattice structure. PNA is a DNA-like polymer with the pentose sugar backbone replaced with peptide backbone like the one proteins have.[75] The backbone has a repeat of the unit N-(2-aminoethyl)-glycine. Figure 4.35 compares the backbone structure of PNA with that of DNA. The reason they are using PNA within the DNA strands is that the backbone of PNA is uncharged. Normally, the crossover points within the double-crossover molecules have the charged phosphate groups in DNA 
backbone in close proximity, and thus require the presence of the divalent ion $\mathrm{Mg}^{2+}$ to form. By removing the charged backbone around the crossover points, the formation of double crossover molecules no longer needs the additional stabilization of the divalent cation, and a gold-particle-friendly sodium containing buffer can be used for annealing. It is possible that this method will remove all problems with non-specific binding and aggregation of the gold.

Initial results from the lab of Prof. Seeman have looked promising. They have TEM images showing labeled individual tiles with four gold nanoparticles apiece. ${ }^{13}$

\subsection{Project Conclusions}

From our portion of the lattice-based nanocrystal patterning experiments, we drew a number of conclusions:

- Making lattices required extremely pure DNA.

- Lattices could be easily damaged by the AFM sample preparation process.

- Gold nanoparticles aggregated in the presence of $6 \mathrm{mM} \mathrm{Mg}^{2+}$ or in the presence of $30 \%$ isopropanol.

- Modifying lattices with nanocrystals would require either removing the need for $\mathrm{Mg}^{2+}$, or using nanoparticles stable in the presence of $\mathrm{Mg}^{2+}$ or $30 \%$ isopropanol. We considered using semiconductor nanocrystals coated with the siloxane layer, but that technology was also labor-intensive, and not perfected until late in the course of this

\footnotetext{
${ }^{13}$ Nadrian Seeman, unpublished data
} 
project.

- Lattices left for longer than the reported two days would continue to grow to as much as 20 or 30 microns in length while remaining only a few microns wide.

- Two seemingly identical lattice reactions would behave differently during annealing. Some would form large lattices, while others would fail to form at all. The reason for this difference is still unclear.

- It was necessary to search a large area of the AFM mica sample surface to find the lattices if they were present. 


\section{Chapter 5}

\section{Conclusion}

This dissertation has described two projects we pursued to use DNA as a templating material for making nanocrystal arrangments. We have used synthetically branched DNA structures to introduce greater complexity to existing technology to make small discrete structures of dimers and trimers. The addition of the branched structures made interpreting the migration of the resulting gel bands more challenging, but with some refinements, it is likely we will make symmetric branched gold trimer and tetramer structures. Other structures with greater complexity may be more difficult to make without a fundamental change in our preparation techniques.

We also have worked toward patterning nanocrystals into ordered arrays using DNA technology developed by the group of Prof. Nadrian Seeman at New York University. Their design for a DNA lattice required the presence of $\mathrm{Mg}^{2+}$, however, and that divalent cation caused the charge-stabilized gold nanoparticles to aggregate or bind to the lattices

non-specifically. A new design for the lattice removes that requirement for magnesium by 
making portions of the DNA strands have an uncharged peptide backbone. With the new magnesium-free design, they may be able to confine the binding of the nanocrystals to the lattices to the specifically intended binding sites, and make ordered nanocrystal arrays.

While pursuing these projects, we have developed many of the techniques that may be part of our future nanostructure-building toolkit. We have also learned the limitations of those techniques. The greatest potential for fast progress in these projects would result from the development of more efficient and reliable ways to prepare large quantities of nanocrystals with only one DNA attached, or from the development of optical techniques (currently in progress) that would allow evaluating the structures using a much smaller reaction amount. Another technique that would make some projects either possible or easier is radioactive labeling of the DNA.

In closing I would like to say that having the opportunity to work on these projects on the forefront of nanotechnology research has been a wonderful challenge. 


\section{Bibliography}

[1] C. J. Loweth, W. B. Caldwell, X. G. Peng, A. P. Alivisatos, and P. G. Schultz. DNA-based assembly of gold nanocrystals. Angewandte Chemie-International Edition, 38(12):1808-1812, 1999.

[2] E. Winfree, F. R. Liu, L. A. Wenzler, and N. C. Seeman. Design and self-assembly of two-dimensional DNA crystals. Nature, 394(6693):539-544, 1998.

[3] F. R. Liu, R. J. Sha, and N. C. Seeman. Modifying the surface features of twodimensional DNA crystals. Journal of the American Chemical Society, 121(5):917-922, 1999.

[4] B. O. Dabbousi, J. RodriguezViejo, F. V. Mikulec, J. R. Heine, H. Mattoussi, R. Ober, K. F. Jensen, and M. G. Bawendi. (cdse)zns core-shell quantum dots: Synthesis and characterization of a size series of highly luminescent nanocrystallites. Journal of Physical Chemistry B, 101(46):9463-9475, 1997.

[5] Juergen Suehnel. Image library of biological macromolecules. http://www.imbjena.de/IMAGE.html, 1999.

[6] G. M. Whitesides, J. P. Mathias, and C. T. Seto. Molecular self-assembly and 
nanochemistry - a chemical strategy for the synthesis of nanostructures. Science, 254(5036):1312-1319, 1991.

[7] D. Gerion, F. Pinaud, S. C. Williams, W. J. Parak, D. Zanchet, S. Weiss, and A. P. Alivisatos. Synthesis and properties of biocompatible water-soluble silicacoated CdSe/ZnS semiconductor quantum dots. Journal of Physical Chemistry B, 105(37):8861-8871, 2001.

[8] Gerald Karp. Cell and Molecular Biology: Concepts and Experiments. John Wiley \& Sons, Inc., New York, 3rd edition, 2002.

[9] N. Ban, P. Nissen, J. Hansen, P. B. Moore, and T. A. Steitz. The complete atomic structure of the large ribosomal subunit at 2.4 angstrom resolution. Science, 289(5481):905919, 2000.

[10] MALDI Basics: Matrix-Assisted Laser Desorption Ionization (MALDI). http://www.srsmaldi.com/Maldi/Maldi.html, 11/3/03 2003.

[11] M. S. Shchepinov, K. U. Mir, J. K. Elder, M. D. Frank-Kamenetskii, and E. M. Southern. Oligonucleotide dendrimers: stable nano-structures. Nucleic Acids Research, 27(15):3035-3041, 1999.

[12] C. A. Mirkin, R. L. Letsinger, R. C. Mucic, and J. J. Storhoff. A DNA-based method for rationally assembling nanoparticles into macroscopic materials. Nature, 382(6592):607609, 1996.

[13] N. C. Seeman and N. R. Kallenbach. Design of immobile nucleic-acid junctions. Biophysical Journal, 44(2):201-209, 1983. 
[14] Bio-Rad Laboratories, Life Science Group. Model 491 Prep Cell Instruction Manual.

[15] A. I. H. Murchie, R. M. Clegg, E. Vonkitzing, D. R. Duckett, S. Diekmann, and D. M. J. Lilley. Fluorescence energy-transfer shows that the 4-way DNA junction is a right-handed cross of antiparallel molecules. Nature, 341(6244):763-766, 1989.

[16] Harvard Apparatus. manual for electrodialyzer. http://www.harvardapparatus.com.

[17] S. J. Xiao, F. R. Liu, A. E. Rosen, J. F. Hainfeld, N. C. Seeman, K. Musier-Forsyth, and R. A. Kiehl. Selfassembly of metallic nanoparticle arrays by DNA scaffolding. Journal of Nanoparticle Research, 4(4):313-317, 2002.

[18] G. Stix. Nanotechnology is all the rage. but will it meet its ambitious goals? and what the heck is it? Scientific American, 285(3):32-37, 2001.

[19] A. P. Alivisatos. Semiconductor clusters, nanocrystals, and quantum dots. Science, 271(5251):933-937, 1996.

[20] R. Elghanian, J. J. Storhoff, R. C. Mucic, R. L. Letsinger, and C. A. Mirkin. Selective colorimetric detection of polynucleotides based on the distance-dependent optical properties of gold nanoparticles. Science, 277(5329):1078-1081, 1997.

[21] J. J. Storhoff, R. Elghanian, R. C. Mucic, C. A. Mirkin, and R. L. Letsinger. One-pot colorimetric differentiation of polynucleotides with single base imperfections using gold nanoparticle probes. Journal of the American Chemical Society, 120(9):1959-1964, 1998.

[22] Y. Maeda, T. Nakamura, K. Uchimura, T. Matsumoto, H. Tabata, and T. Kawai. 
Controlled conjugation of nanoparticles with single stranded DNA. Journal of Vacuum Science $\&$ Technology B, 17(2):494-496, 1999.

[23] J. J. Storhoff, A. A. Lazarides, R. C. Mucic, C. A. Mirkin, R. L. Letsinger, and G. C. Schatz. What controls the optical properties of DNA-linked gold nanoparticle assemblies? Journal of the American Chemical Society, 122(19):4640-4650, 2000.

[24] L. He, M. D. Musick, S. R. Nicewarner, F. G. Salinas, S. J. Benkovic, M. J. Natan, and C. D. Keating. Colloidal au-enhanced surface plasmon resonance for ultrasensitive detection of DNA hybridization. Journal of the American Chemical Society, 122(38):9071-9077, 2000.

[25] F. Patolsky, K. T. Ranjit, A. Lichtenstein, and I. Willner. Dendritic amplification of DNA analysis by oligonucleotide-functionalized au-nanoparticles. Chemical Communications, (12):1025-1026, 2000.

[26] S. J. Park, A. A. Lazarides, C. A. Mirkin, P. W. Brazis, C. R. Kannewurf, and R. L. Letsinger. The electrical properties of gold nanoparticle assemblies linked by DNA. Angewandte Chemie-International Edition, 39(21):3845-+, 2000.

[27] C. M. Niemeyer, B. Ceyhan, S. Gao, L. Chi, S. Peschel, and U. Simon. Site-selective immobilization of gold nanoparticles functionalized with DNA oligomers. Colloid and Polymer Science, 279(1):68-72, 2001.

[28] Y. Maeda, H. Tabata, and T. Kawai. Two-dimensional assembly of gold nanoparticles with a DNA network template. Applied Physics Letters, 79(8):1181-1183, 2001. 
[29] C. M. Niemeyer and B. Ceyhan. DNA-directed functionalization of colloidal gold with proteins. Angewandte Chemie-International Edition, 40(19):3685-+, 2001.

[30] J. J. Storhoff, R. Elghanian, C. A. Mirkin, and R. L. Letsinger. Sequence-dependent stability of DNA-modified gold nanoparticles. Langmuir, 18(17):6666-6670, 2002.

[31] D. Zanchet, C. M. Micheel, W. J. Parak, D. Gerion, S. C. Williams, and A. P. Alivisatos. Electrophoretic and structural studies of DNA-directed Au nanoparticle groupings. Journal of Physical Chemistry B, 106(45):11758-11763, 2002.

[32] BBInternational. Technical Data Sheet for Gold Colloids.

[33] M. A. Hines and P. GuyotSionnest. Synthesis and characterization of strongly luminescing zns-capped cdse nanocrystals. Journal of Physical Chemistry, 100(2):468-471, 1996.

[34] M. Bruchez, M. Moronne, P. Gin, S. Weiss, and A. P. Alivisatos. Semiconductor nanocrystals as fluorescent biological labels. Science, 281(5385):2013-2016, 1998.

[35] W. J. Parak, D. Gerion, D. Zanchet, A. S. Woerz, T. Pellegrino, C. Micheel, S. C. Williams, M. Seitz, R. E. Bruehl, Z. Bryant, C. Bustamante, C. R. Bertozzi, and A. P. Alivisatos. Conjugation of DNA to silanized colloidal semiconductor nanocrystalline quantum dots. Chemistry of Materials, 14(5):2113-2119, 2002.

[36] S. A. Empedocles, D. J. Norris, and M. G. Bawendi. Photoluminescence spectroscopy of single CdSe nanocrystallite quantum dots. Physical Review Letters, 77(18):3873-3876, 1996. 
[37] O. Kulakovich, N. Strekal, A. Yaroshevich, S. Maskevich, S. Gaponenko, I. Nabiev, U. Woggon, and M. Artemyev. Enhanced luminescence of CdSe quantum dots on gold colloids. Nano Letters, 2(12):1449-1452, 2002.

[38] M. L. Brongersma, J. W. Hartman, and H. A. Atwater. Electromagnetic energy transfer and switching in nanoparticle chain arrays below the diffraction limit. Physical Review B, 62(24):R16356-R16359, 2000.

[39] S. A. Maier, M. L. Brongersma, P. G. Kik, and H. A. Atwater. Observation of near-field coupling in metal nanoparticle chains using far-field polarization spectroscopy. Physical Review B, 65(19), 2002.

[40] John McMurry. Organic Chemistry. Brooks/Cole Publishing Company, Pacific Grove, CA, 3rd edition, 1992.

[41] J. D. Watson and F. H. C. Crick. Molecular structure of nucleic acids - a structure for deoxyribose nucleic acid. Nature, 171(4356):737-738, 1953.

[42] G. Michael Blackburn and Michael J. Gait, editors. Nucleic Acids in Chemistry and Biology. Oxford University Press, New York, 2nd edition, 1996.

[43] E. Dujardin, L. B. Hsin, C. R. C. Wang, and S. Mann. Dna-driven self-assembly of gold nanorods. Chemical Communications, (14):1264-1265, 2001.

[44] C. M. Niemeyer, W. Burger, and J. Peplies. Covalent dna - streptavidin conjugates as building blocks for novel biometallic nanostructures. Angewandte Chemie-International Edition, 37(16):2265-2268, 1998. 
[45] E. Braun, Y. Eichen, U. Sivan, and G. Ben-Yoseph. Dna-templated assembly and electrode attachment of a conducting silver wire. Nature, 391(6669):775-778, 1998.

[46] A. P. Alivisatos, K. P. Johnsson, X. G. Peng, T. E. Wilson, C. J. Loweth, M. P. Bruchez, and P. G. Schultz. Organization of 'nanocrystal molecules' using DNA. Nature, 382(6592):609-611, 1996.

[47] M. L. Sauthier, R. L. Carroll, C. B. Gorman, and S. Franzen. Nanoparticle layers assembled through dna hybridization: Characterization and optimization. Langmuir, 18(5):1825-1830, 2002.

[48] Harvey Lodish, David Baltimore, Arnold Berk, S. Lawrence Zipursky, Paul Matsudaira, and James Darnell. Molecular Cell Biology. Scientific American Books, New York, 3rd edition, 1995.

[49] M. H. Caruthers. Chemical synthesis of DNA. Journal of Chemical Education, $66(7): 577-580,1989$.

[50] Victor A. Bloomfield, Donald M. Crothers, and Jr. Tinoco, Ignacio. Nucleic Acids: Structures, Properties, and Functions. University Science Books, Sausalito, CA, 2000.

[51] D. Gerion, W. J. Parak, S. C. Williams, D. Zanchet, C. M. Micheel, and A. P. Alivisatos. Sorting fluorescent nanocrystals with DNA. Journal of the American Chemical Society, 124(24):7070-7074, 2002.

[52] L. M. Demers, D. S. Ginger, S. J. Park, Z. Li, S. W. Chung, and C. A. Mirkin. Direct patterning of modified oligonucleotides on metals and insulators by dip-pen nanolithography. Science, 296(5574):1836-1838, 2002. 
[53] G. Schmid. Large clusters and colloids - metals in the embryonic state. Chemical Reviews, 92(8):1709-1727, 1992.

[54] M. A. Hayat. Colloidal Gold: Principles, Methods, and Applications, volume 1. Academic Press, San Diego, 1989.

[55] W. J. Parak, T. Pellegrino, C. M. Micheel, D. Gerion, S. C. Williams, and A. P. Alivisatos. Conformation of oligonucleotides attached to gold nanocrystals probed by gel electrophoresis. Nano Letters, 3(1):33-36, 2003.

[56] W. J. Parak, D. Gerion, T. Pellegrino, D. Zanchet, C. Micheel, S. C. Williams, R. Boudreau, M. A. Le Gros, C. A. Larabell, and A. P. Alivisatos. Biological applications of colloidal nanocrystals. Nanotechnology, 14(7):R15-R27, 2003.

[57] Applied Biosystems. Expedite 8900 Nucleic Acid Synthesis System User's Guide, 2001.

[58] J. F. Ramalho Ortigao and R. Rosch. Interactiva: Access to the virtual laboratory. http://www.interactiva.de/knowledge/nucleicchem/solidphase.html, 6/23/00 2000.

[59] Andrew Ellington and Jr. Pollard, Jack D. Synthesis and Purification of Oligonucleotides. Current Protocols in Molecular Biology. John Wiley \& Sons, Inc., New York, 1998.

[60] Glen Research. User Guide to DNA Purification. http://www.glenres.com/Catalog/purification.html.

[61] D. M. Hawcroft. Electrophoresis: The Basics. IRL Press at Oxford University Press, New York, 1997. 
[62] Douglas A. Skoog, Donald West, and F. James Holler. Fundamentals of Analytical Chemistry. Saunders College Publishing, San Diego, 6th edition, 1992.

[63] BioWhittaker Molecular Applications. GelStar Nucleic Acid Gel Stain Instructions.

[64] Glen Research. User Guide to DNA Modification. http://www.glenres.com.

[65] M. S. Shchepinov, I. A. Udalova, A. J. Bridgman, and E. M. Southern. Oligonucleotide dendrimers: synthesis and use as polylabelled DNA probes. Nucleic Acids Research, 25(22):4447-4454, 1997.

[66] D. Zanchet, C. M. Micheel, W. J. Parak, D. Gerion, and A. P. Alivisatos. Electrophoretic isolation of discrete $\mathrm{Au}$ nanocrystal/DNA conjugates. Nano Letters, $1(1): 32-35,2001$.

[67] N. C. Seeman. Nucleic-acid junctions and lattices. Journal of Theoretical Biology, 99(2):237-247, 1982.

[68] N. C. Seeman. Interactive design and manipulation of macromolecular architecture utilizing nucleic-acid junctions. Journal of Molecular Graphics, 3(2):34-39, 1985.

[69] N. C. Seeman. Denovo design of sequences for nucleic-acid structural-engineering. Journal of Biomolecular Structure $\&$ Dynamics, 8(3):573-581, 1990.

[70] C. D. Mao, W. Q. Sun, and N. C. Seeman. Designed two-dimensional DNA holliday junction arrays visualized by atomic force microscopy. Journal of the American Chemical Society, 121(23):5437-5443, 1999. 
[71] R. Holliday. Mechanism for gene conversion in fungi. Genetical Research, 5(2):282-304, 1964.

[72] G. Binnig, C. F. Quate, and C. Gerber. Atomic force microscope. Physical Review Letters, 56(9):930-933, 1986.

[73] H. G. Hansma, J. Vesenka, C. Siegerist, G. Kelderman, H. Morrett, R. L. Sinsheimer, V. Elings, C. Bustamante, and P. K. Hansma. Reproducible imaging and dissection of plasmid DNA under liquid with the atomic force microscope. Science, 256(5060):1180$1184,1992$.

[74] J. Vesenka, M. Guthold, C. L. Tang, D. Keller, E. Delaine, and C. Bustamante. Substrate preparation for reliable imaging of DNA-molecules with the scanning force microscope. Ultramicroscopy, 42:1243-1249, 1992. Part B.

[75] Eurogentec. Peptide Nucleic Acid (PNA). http://www.eurogentec.be/module/FileLib/oligo_16.pdf. 


\section{Appendix A}

\section{Glossary}

This glossary is an attempt to explain and summarize some of the jargon in this dissertation. It is not a very exhaustive list, so I apologize if the word you needed to know is not here. Some of these words might seem obvious to some people. I hope those people will just ignore the words they already know. ${ }^{1}$

3' end Each 2'-deoxyribose sugar in a DNA molecule has five carbons, each numbered 1' to 5'. A strand of DNA has two distinguishable ends. The end where an additional nucleotide, if it were added, would attach to the 3' carbon on the last sugar is called the "3' end." Figure A.1

5' end Each 2'-deoxyribose sugar in a DNA molecule has five carbons, each numbered 1' to 5'. A strand of DNA has two distinguishable ends. The end where an additional nucleotide, if it were added, would attach to the 5' carbon on the last sugar is called the "5' end." Figure A.1 shows the sugar labeling.

\footnotetext{
${ }^{1}$ I have attempted to check the information here, but I apologize for any possible errors.
} 


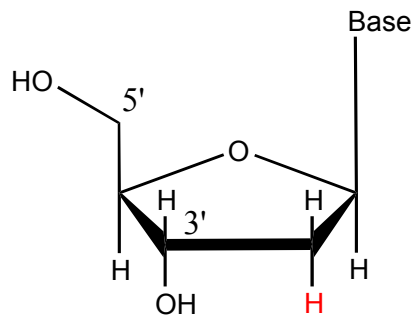

Figure A.1: 5' and 3' ends labeled on a 2'-deoxyribonucleoside

acetic anhydride Chemical shown in Figure A.2.

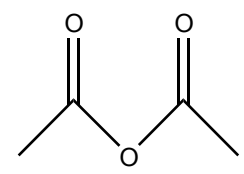

Figure A.2: Acetic anhydride molecule.

adenine One of the four bases found in DNA. Figure A.3 shows a nucleoside with adenine.

AFM Abbreviation for an atomic force microscope. See Figure 4.13.

amine Reactive nitrogen-containing group that can also participate in hydrogen-bonding. See Figure A.4.

anneal In this context, to place complementary DNA strands into a solution together under conditions which promote formation of double helices. Usually the annealing reaction takes place over the course of hours or days. It can happen at a single temperature, or with changing temperatures, and it requires the presence of some salt in the annealing buffer. 


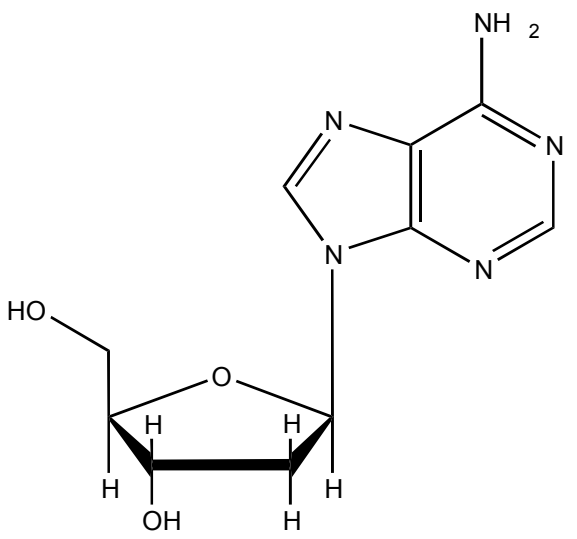

Figure A.3: 2'-Deoxyadenosine

$$
\mathrm{R}-\mathrm{NH} \text { : }
$$

Figure A.4: Amine group.

backbone (in DNA) The covalently-linked sugars in a DNA strand. See Figure A.5.

band A place in the gel that contains things of a discrete type, such that they travel at the same rate through the gel during electrophoresis. Depending on what is in the band, the band contents may have to be stained to be seen.

colloid A suspension of very small particles. The small particles do not dissolve in the solution, but do remain suspended in it.

conjugates In the context of this dissertation, a term refering to nanoparticles or nanocrystals having DNA attached to them.

contact mode A way of creating an AFM image where the tip is resting on the surface, and drags across it as it creates a topographic image. 


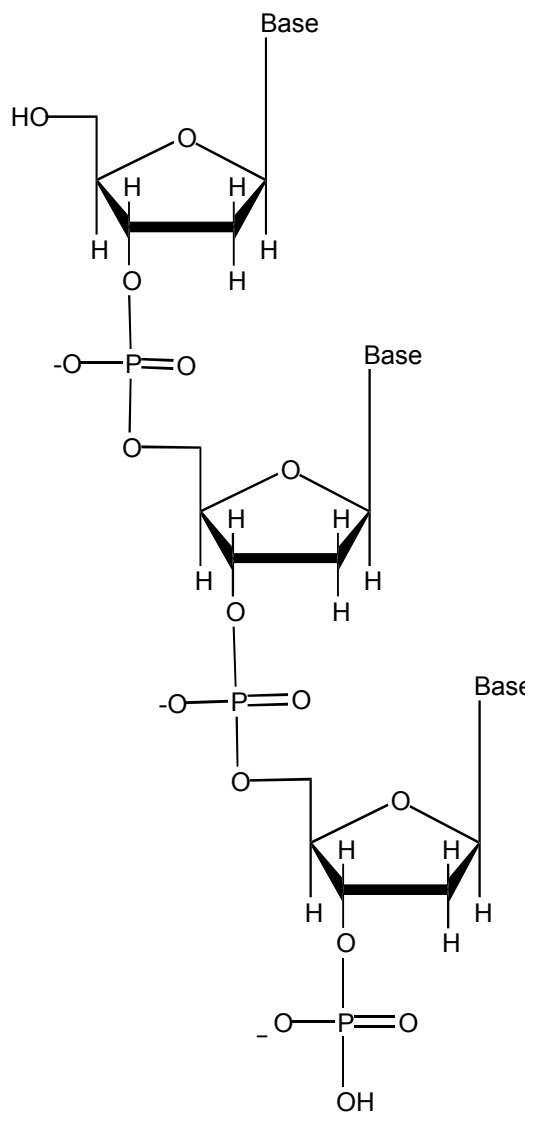

Figure A.5: Figure showing the sugar backbone of the DNA strand.

criton Older term for a "vocabulary element."

cytosine One of the four bases found in DNA. Figure A.6 shows a nucleoside with cytosine.

denaturing PAGE Polyacrylamide gel electrophoresis where the gel medium contains a denaturing agent (usually urea) to disrupt non-covalent bonding in the molecules.

dimethoxytrityl group Also known as a "DMT" group. A protecting group for the 5' hydroxyl group of the ribose during solid-phase chemical synthesis of DNA. Figure A.7 shows its chemical structure. 


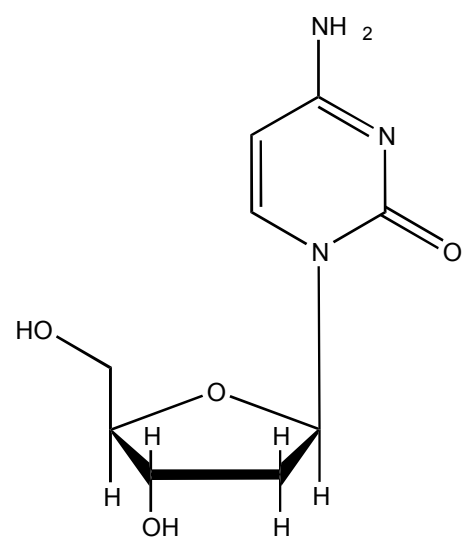

Figure A.6: 2'-Deoxycytidine

double-crossover molecule A DNA structure invented in Prof. Seeman's research group that has structural rigidity that results from the presence of two Holliday Junction "crossover" points for the DNA strands in the structure. For the DNA lattices, double-crossover molecules served as the component tiles. Four to nine DNA strands comprised the double-crossover molecules we used to make lattices.

doubling synthon A phosphoramidite that allows the automated synthesizer to split the DNA backbone into two strands, mid-synthesis. Figure 2.21 shows its structure.

electrophoresis A process of separating samples by size, charge, and shape, where the sample moves in buffer through a porous sieving medium, driven by an electric field.

ethanol precipitation The process of precipitating DNA from a water-based solution using by introducing a large volume of ethanol to the solution, and then freezing at dry-ice temperature.

extraction In the context of this dissertation, to remove the contents of a gel band from 


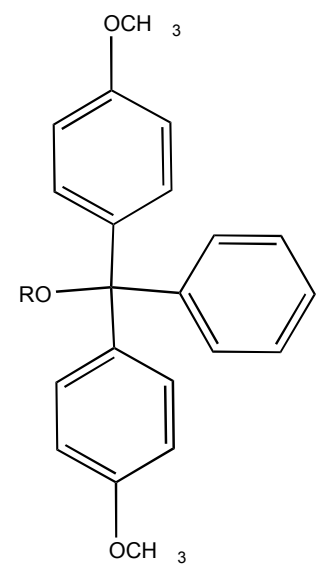

Figure A.7: Dimethoxytrityl group that protects an hydroxyl group as an ether.

the band so it can be used.

guanine One of the four bases found in DNA. Figure A.8 shows a nucleoside with guanine.

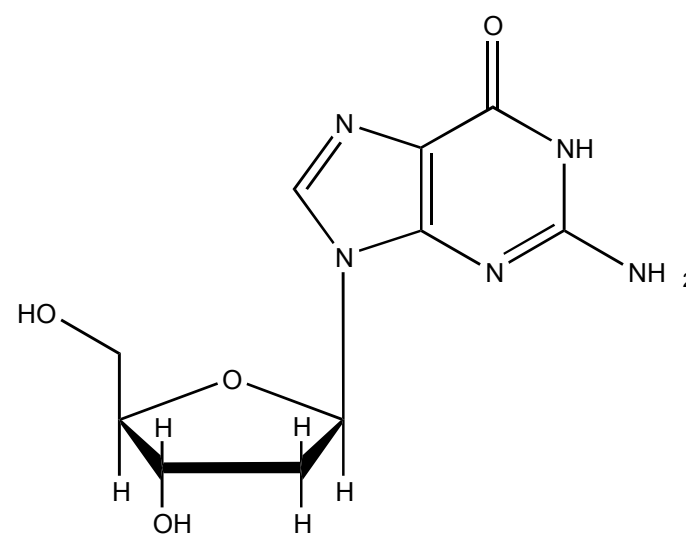

Figure A.8: 2'-Deoxyguanosine

heterocyclic Cyclic organic compounds containing at least atom other than carbon in their cyclic portion. 
Holliday Junction A four-way junction that occurs naturally in DNA during recombination. In nature, this junction migrates quickly along the DNA strands because of the sequence symmetry present. Here, we used stationary Holliday Junctions. See Figure 4.2 .

hybridization Here, attaching something to something else. We use this term mostly to describe attaching a DNA strand to its complement(s) by annealing.

hydroxyl An alcohol, or "-OH" chemical group.

kinase Naturally-occurring enzymes that can attach a phophoryl groups to a DNA strand.

lattice modification Attaching something (such as DNA, proteins, or nanocrystals) to the surface of a DNA lattices.

MALDI Matrix-Assisted Laser Desorption Ionization. See Figure 2.3.3.

native PAGE Polyacrylamide gel electrophoresis where conditions are carefully maintained so as to preserve non-covalent bonds such as hydrogen-bonded base pairs.

nanometer $1 \times 10^{-9}$ meters, or $1 / 1,000,000$ th of a millimeter.

pentose A sugar having five carbon atoms.

phosphodiester bond The type of phosphate linkage between successive sugars in the DNA backbone. See Figure A.9.

phosphoramidites Chemical reagents that are protected synthetic precursors for the solid-phase synthesis of DNA. 


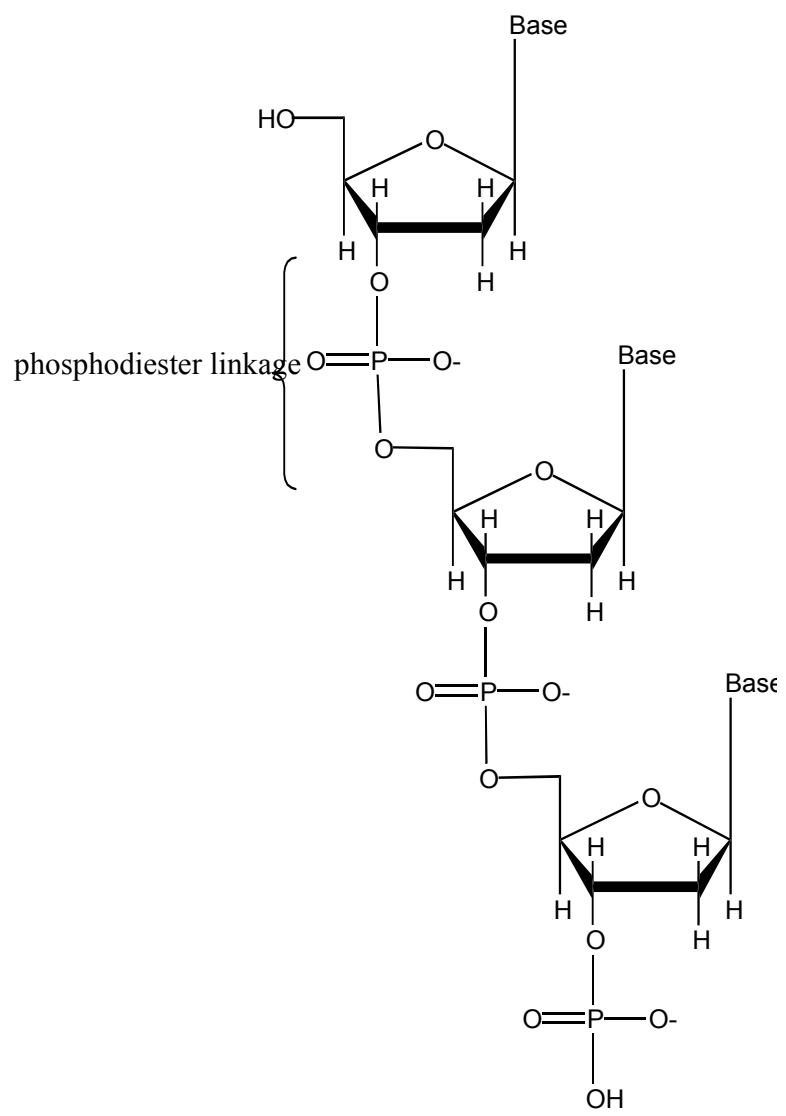

Figure A.9: Figure showing the phosphodiester linkage in a DNA strand backbone.

piezocrystal A crystal that expands or contracts along certain axes when a voltage is applied.

plasmid A circular ring of several thousand DNA base-pairs found independently of the chromosome in bacteria.

polymer A chemical composed of a chain of repeated units.

polymerase A natural enzyme that synthesizes DNA in biological environment using a template strand and nucleotides. 
purines One of two types of DNA bases, having two conjoined rings. See adenine and guanine.

pyrimidines One of two types of bases found in DNA, having a single six-membered ring. See cytosine and thymine.

recirculating To flow a fluid continuously, recycling it. We used this method to refresh buffer during long electrophoresis runs.

RNA A polymer similar to DNA labeled with the four bases, adenine, guanine, cytosine, and uracil (instead of thymine). The main difference between RNA and DNA is that RNA has a 2'hydroxyl group on its pentose sugars. See Figure A.10. RNA takes a different helical form than DNA.

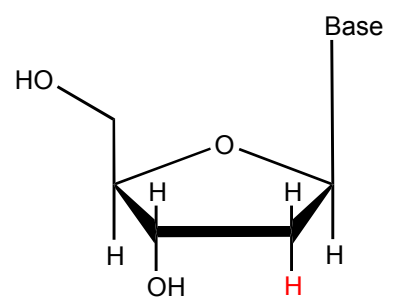

DNA nucleoside

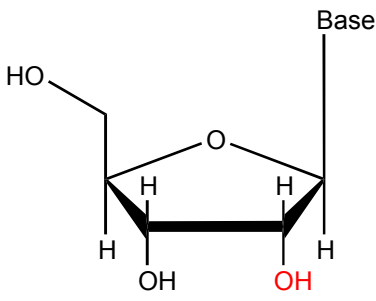

RNA nucleoside

Figure A.10: Figure comparing DNA and RNA nucleosides. The RNA one has a 2' hydroxyl group.

running buffer The buffer used for running gels in an electrophoresis chamber. It carries charge and prevents $\mathrm{pH}$ changes due to water hydrolysis at the electrodes. We usually used either Tris-acetate-EDTA, or Tris-borate-EDTA for our gels.

scanning probe microscopy A type of microscopy that can achieve nanometer-scale res- 
olution by scanning some type of probe across a surface.

self-assembly A process whereby achieving thermodynamic equilibrium causes component structures to non-covalently bond together to form a larger structure.

self-complementary In DNA, a base sequence that happens to be the complement of itself. An short sequence example would be "5'-AGCT-3'."

sol A specific type of colloid where solid particles are suspended in a liquid.

split photodiode A device, split into sections, that detects incident light.

stain, DNA DNA is not generally visible to the naked eye, so to image it or extract it from gels, we would stain it using an intercalating DNA stain. The stains are generally large aromatic structures that slip between base-pairs in the DNA strand. They are bound by aromatic stacking forces.

synthesizer A computerized machine that controls the synthesis of DNA.

tapping mode An AFM image collection mode where the tip is rastered across the surface while it rapidly oscillates. This method reduces tip-sample dragging forces.

TEM Abbreviation for transmission electron microscopy. This type of microscope images a sample using a focused beam of electrons.

thymine One of the four bases found in DNA. This base is only found in DNA, not in RNA. Figure A.11 shows a nucleoside with thymine.

tile In this dissertation, a double-crossover molecule that is a building component for the 


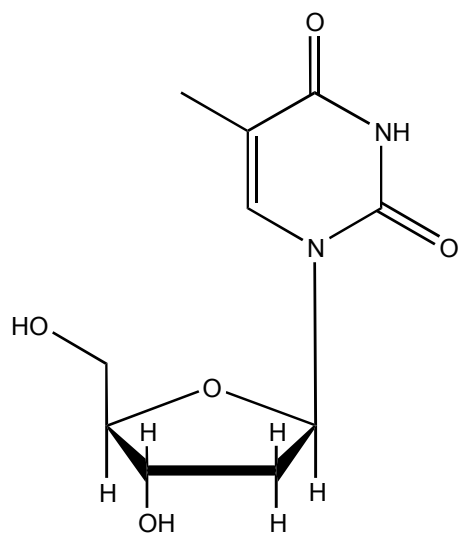

Figure A.11: 2'-Deoxythymidine

self-assembly of DNA lattices. More generally, it is the unit component for any two-dimensional lattice system.

topography Location-mapped height information about a sample or surface.

trimer, DNA A synthetically-branched piece of DNA.

vocabulary element Overlapping units of code corresponding to short segments of the DNA base sequence in a DNA strand. Prof. Nadrian Seeman's "Sequin" program uses the construct of "vocabulary elements" to verify a sequence's lack of unintended base-pairing.

Watson-Crick base pairing The type of base-pairing, "A" with "T" and "G" with "C," that Watson and Crick discovered made up the natural double helix of DNA. Figure 1.3 shows these base pairs. 


\section{Appendix B}

\section{List of Publications and}

\section{Presentations}

- W. J. Parak, D. Gerion, T. Pellegrino, D. Zanchet, C. M. Micheel, S. C. Williams, R. Boudreau, M. A. Le Gros, C. A. Larabell, and A. P. Alivisatos, "Biological Applications of colloidal nanocrystals." Nanotechnology 14 (7), July 2003: R15-R27.

- W. J. Parak, T. Pellegrino, C. M. Micheel, D. Gerion, S. C. Williams, and A. P. Alivisatos, "Conformation of oligonucleotides attached to gold particles probed by gel electrophoresis." Nanoletters 3 (1), January 2003: 33-36.

- S. C. Williams, S. L. Goh, C. M. Micheel, D. Zanchet, W. J. Parak, D. Gerion, and A. P. Alivisatos. "Producing discrete and more complex nanoparticle arrangements using asymmetric branched DNA trimers." Oral Presentation, Amer. Chem. Soc. National Meeting, New Orleans, LA, March 2003. 
- D. Zanchet, C. M. Micheel, W. J. Parak, D. Gerion, S. C. Williams, and A. P. Alivisatos. "Electrophoretic and Structural Studies of DNA-directed Au Nanoparticle Groupings." Journal of Physical Chemistry B 106 (45),14 November 2002: 1175811763.

- D. Gerion, W. J. Parak, S. C. Williams, D. Zanchet, C. M. Micheel, A. P. Alivisatos. "Sorting fluorescent nanocrystals with DNA." Journal of the American Chemical Society, 124 (24), 19 June 2002: 7070-4.

- W. J. Parak, R. Boudreau, M. Le Gros, D. Gerion, D. Zanchet, C. M. Micheel, S. C. Williams, A. P. Alivisatos, C. Larabell. "Cell motility and metastatic potential studies based on quantum dot imaging of phagokinetic tracks." Advanced Materials 14 (12): 18 June 2002: 882-5.

- W. J. Parak, D. Gerion, D. Zanchet, A. S. Woerz, T. Pellegrino, C. M. Micheel, S. C. Williams, M. Seitz, R. E. Bruehl, Z. Bryant, C. Bustamante, C. R. Bertozzi, A. P. Alivisatos. "Conjugation of DNA to silanized colloidal semiconductor nanocrystalline quantum dots." Chemical Materials 14 (5), May 2002: 2113-2119.

- D. Gerion, F. Pinaud, S. C. Williams, W. J. Parak, D. Zanchet, S. Weiss, A. P. Alivisatos "Synthesis and properties of biocompatible water-soluble silica-coated CdSe/ZnS semiconductor quantum dots." Journal of Physical Chemistry B, 105 (37), 20 Sept. 2001: 8861-71.

- S. C. Williams, S. Cook, M. A. Hoffbauer. "Absolute Momentum Accommodation Measurements Using a Specialized Torsion Balance." Poster Presentation. SERS Pro- 
gram, August 1996.

- D. G. J. Sutherland, J. A. Carlisle, P. Elliker, G. Fox, T. W. Hagler, I. Jiminez, H. W. Lee, K. Pakbaz, L. J. Terminello, S. C. Williams, F. J. Himpsel, D. K. Shuh, W. M. Tong, J. J. Jia, T. A. Callcott, and D. L. Ederer. "Photo-oxidation of electroluminescent polymers studied by core-level photoabsorption spectroscopy." Applied Physics Letters, 68 (15), 8 Apr. 1996: 2046-8. 